\title{
Analysis of Hybrid Petri nets with Random Discrete Events
}

Hamed Ghasemieh

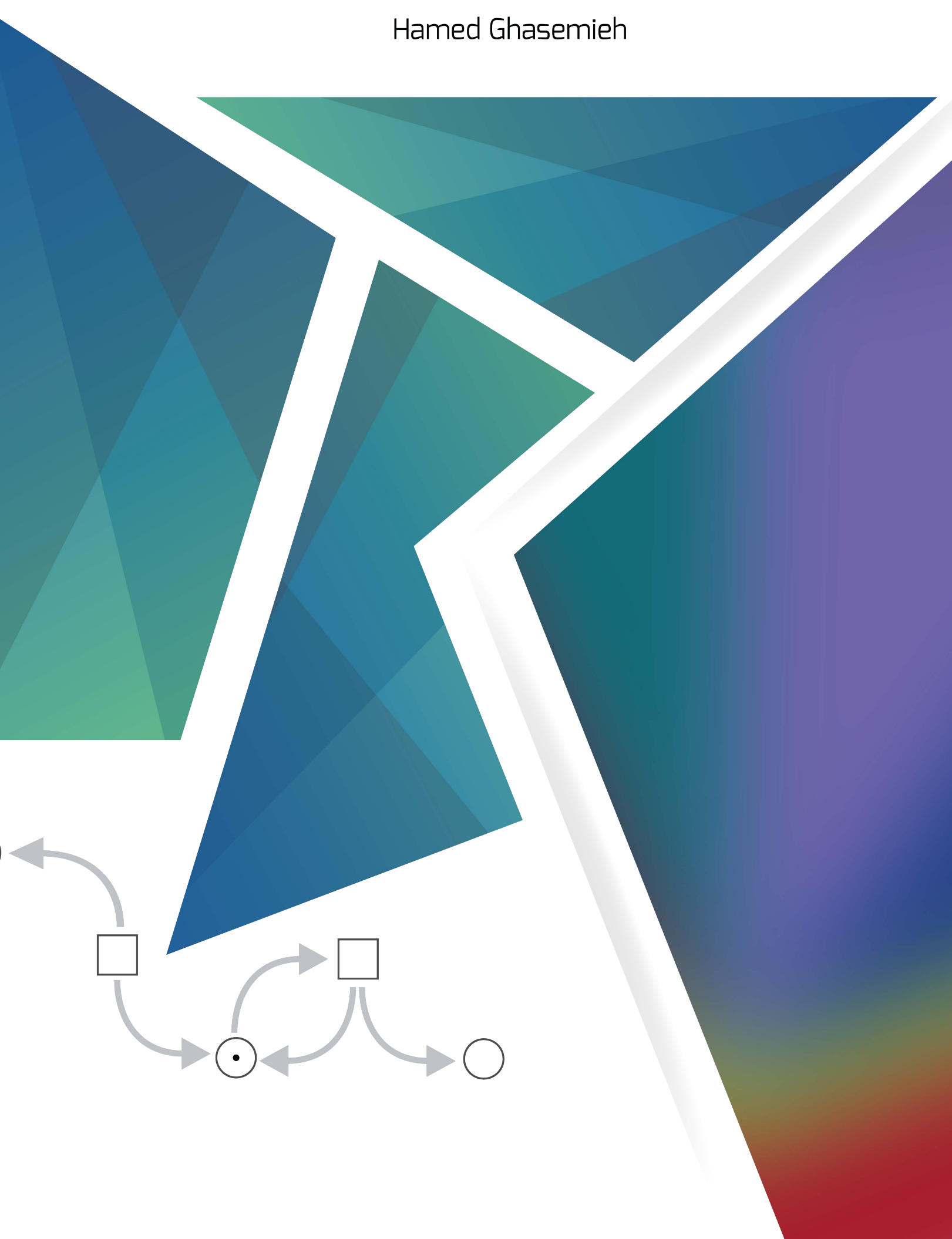


Analysis of Hybrid Petri nets with

Random Discrete Events

Hamed Ghasemieh 
Graduation committee:

Chairman:

Promoter:

Promoter:

Members:

Prof. dr. ir. J.P. Katoen

Dr. ir. R. Langerak

Prof. dr. G. Ciardo

Prof. dr. M. Gribaudo

Prof. dr. ing. H. Hermanns
Prof. dr. P.M.G. Apers

Prof. dr. ir. B.R.H.M. Haverkort

Prof. dr. A.K.I. Remke

\begin{abstract}
University of Twente University of Twente Iowa State University Politecnico di Milano Universität Saarland
\end{abstract}

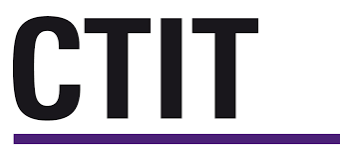

CTIT Ph.D. - thesis Series No. 17-420

Centre for Telematics and Information Technology University of Twente P.O. Box 217, 7500 AE Enschede, The Netherlands.

ISBN 978-90-365-4257-9

ISSN 1381-3617 (CTIT Ph.D. thesis Series No. 17-420)

DOI 10.3990/1.9789036542579

https://dx.doi.org/10.3990/1.9789036542579

Typeset with $\mathrm{AT}_{\mathrm{E}} \mathrm{X}$.

Cover design: Mahdi Beheshti

Copyright (C)2017 Hamed Ghasemieh, Enschede, The Netherlands

All rights reserved. No part of this book may be reproduced or transmitted, in any form or by any means, electronic or mechanical, including photocopying, microfilming, and recording, or by any information storage or retrieval system, without the prior written permission of the author. 


\title{
ANALYSIS OF HYBRID PETRI NETS WITH RANDOM DISCRETE EVENTS
}

\author{
DISSERTATION
}

to obtain

the degree of doctor at the University of Twente, on the authority of the rector magnificus,

Prof. dr. T.T.M. Palstra, on account of the decision of the graduation committee, to be publicly defended

on Friday the 3rd of February 2017 at 14:45

by

Hamed Ghasemieh

born on the 14th of June, 1985

in Tehran, Iran 
This dissertation has been approved by:

Prof. dr. ir. B.R.H.M Haverkort (promoter)

Prof. dr. A.K.I. Remke (promoter) 


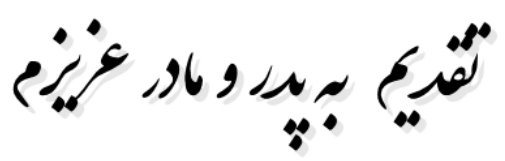

> 

More and more, our society and economy rely on the correct operation of, often hidden, critical infrastructures. These infrastructures such as the power grid and water and gas distribution networks, play an important role in our everyday life. Continuous supply of services from these assets is essential for people, organizations, and for the security and economy of our society. It is of substantial value to know or estimate how quickly such systems recover to acceptable levels of service after the occurrence of failures, natural disasters, e.g., fire, earthquakes, or cyber-attacks.

Critical infrastructure are naturally hybrid, i.e., one needs both discrete and continuous quantities to realistically describe their behaviour. Moreover, in many modern applications there is an intrinsic uncertainty. This is particularly true for dependability analysis of critical infrastructures, where one must model the occurrence of failure and repair in a system.

In this dissertation we propose the use of an extended version of stochastic hybrid Petri nets. This modelling formalism combines discrete and continuous quantities with random discrete events. Furthermore, Petri nets provide a high level and easy-to-understand formalism. Particularly, we consider so called Hybrid Petri nets with General transitions (HPnG). The term general transition refers to the arbitrary nature of probability distribution that can be associated with stochastic variables in this model. HPnGs form a restricted subclass of stochastic hybrid models.

The arbitrary nature of random discrete events in HPnGs is the main challenge for their analysis. We tackle the analysis of HPnGs by a conditioning argument on the occurrence times of random discrete events. This idea leads to an efficient generation of the underlying state space, which provides us with a structure such that measures of interest can be computed exactly and effectively. Unfortunately, the exact computation of measures of interests for complex systems described by HPnGs, will be shown to be inefficient. To overcome this, we will also investigate approximation techniques, providing upper and lower bounds for measures of interest. The approximation techniques are based on discretizing the support of stochastic variables. Moreover, by smart generation and exploration of only parts of the state space, we can come up with upper and lower bounds for the given measures of interest. We will also investigate the feasibility of the methods introduced in this thesis, by considering two real-world applications, namely, dependability analysis of a sewage treatment facility, and a model of a smart house. 



\section{ACKNOWLEDGEMENTS}

All the credits goes to God almighty, Who gives proof of His existence through His creation, of His being eternal through the newness of His creation, and through their mutual similarities proves that nothing is similar to Him. Senses cannot touch Him, because of the difference between the Maker and the made, the Limiter and the limited, the Sustainer and the sustained.

Since no one can be thankful of God, unless he is thankful of His creation to whom he owes, this is my duty to mention and express my thankfulness to many people who have helped me in this long journey.

First of all I have to thank Prof. Anne Remke, to whom I owe the most for giving me the opportunity of working on this project. I remember the first day I met her in her office, "this project is like my baby!", she said with lots of enthusiasm in her eyes. Anne was more like a friend to me than a supervisor, this made these 5 years a joyful working period.

This thesis could not be possible without invaluable helps of Prof. Boudewijn Hoverkort. Boudewijn's extensive and broad view of different topics was always helpful in every steps I took for this thesis. He is always able to see the big picture without delving into the details, the ability I still need to learn. I should also thank Prof. Gianfranco Ciardo, for accepting me to work with him in Iowa State University, despite his busy schedule. Gianfranco's help was so substantial that the idea we had during my visit formed an important chapter of my thesis. I also would like to thank my graduation committee for their comments on this thesis.

I have to thank Davood and Bjorn for accepting to be my Paranymphs. Davood, is among the few people I know who have been able to enter different activity areas and still be successful in all of them, he is a physicist, musician, and a very good manager. Bjorn, provided me with lots food for thought, he is a very good Christian and we had lots of fruitful discussion, although I may have had managed to annoy him sometimes :).

I also would like to thank all the DACS members, who provided lots of joy during the five years that I was part of this warm and dynamic group. Especially I have to thank three ladies that I shared my office with them. Anja, who learned the Persian way of saying bless you "Afiat bashe" - and repeating it relentlessly every time I was sneezing (which was quite a lot during springs). Anna, for laughing with her entire face to my usually unfunny and uncanny jokes. Finally, Justyna for watering and taking care of all the plants we had inherited from 
previous residents of the room that we had never met. The last DACS member, but probably the most important person in our group, that I have to express my thankfulness to, is Jeanette, our secretary. She literally knew every thing, every single time I had a question, she has always been helpful, and a real problem solver.

In the time of my stay in Twente, I was fortunate to be one of the co-founders of IrNUT, Iranian Network at University of Twente. This helped me to form bond and friendship with lots of Iranians. I have to say a special thank to Ma hidi and Hadis, (and also little Taha). Mahdi has always been around any time I needed help (given that he could manage to answer his phone). Two times we moved to a new house and he was the most helpful person, and I am pretty sure he has lots of nice memories to tell for that:). Mahdi, I also have to thank you for designing the cover of this thesis. Alireza and Zahra, are kind of friends that anyone wish to have. Alireza is so full of love and passion, and so eager to transfer this to anyone who is lucky enough to be around him. I have to thank Meysam and Hajar (and little Dora) my very lovely friends. Meysam is so enthusiastic about paradigm shifts, and I have to thank him for opening doors to new worlds for me, and making me rethink and reconsider many things. I also like to thank many friends I had in our weekly gathering for Quran study. Without them life could have been really boring.

I have to thank my very good friend Mohammad, a friend one can always rely on, and make sure he will find a way to solve the problem one is dealing with. Mohammad, I wish you and Ana, a prosperous future life. Mojtaba thank you for being always helpful and stress free. I wish you and Sadaf a great life, and several "topol mopol" kids. Siavash, thank you for being so open minded, and respecting all the differences I have in different aspects of life, and also thank you for always being "paye" and never saying no for any activity I was suggesting. Thanks to Mohammad (MoFo) and Neda (and Nikan, and to be available little one), for being such nice and supporting neighbors, and to Morteza and Mozhde, for creating lots of joyful moments.

Speaking of friends, I have to go a bit deeper backward in time. I am a lucky guys for having lots of good friends, with whom I have shared my life for more than 20 years. However, it is unfortunate that now every one of us is living in a different corner of the world. I have to mention our study group, foco of readers, and its group members Hamed in Purdue University, Amir in University of Chicago, and Ali in University of Waterloo. Anyone with a little experience of conducting an activity group, knows how hard it is to coordinate among members. These guys proved things the other way around. We had bi-weekly meetings for more than 4 years, reading several philosophical books, which sometimes could be really hard for students of technical backgrounds. 
Maryam, you are the love of my life, the light of my eyes, and the moon of my nights. Whenever, I am confused I know you are the one I have to turn to, whenever, I am tired you are the one who inject energy into my body and spirit, and whenever I am lost, you are the one I have to seek guidance from. You are the most precious gift for which I have to be thankful of God almighty. The kind of gift who has given me another priceless gift, Reihana, the warmest sun shining on our life, the peace anyone seeks, and a kind of being that looking into her eyes is literally like looking into galaxies. I wish, and pray to God to always keep me reminded, that all these are his blessing and endowment.

My only sister and childhood friend Narges, I know your heart is filled with love, and I am sorry for every single time that I made your beautiful green eyes wet, by leaving you to go abroad. I promise you the time of separation is going to be finished soon, and we will be living close to each other again. Yahya my brother-in-law, I rather to just say brother, though. We know each other for more than 20 years, 12 years of which is prior to becoming family. I have to thank you for all the funny and joyful moments we created together. I also like to thank Maryam's parents for all the support they gave us, and most importantly for trusting my judgments for all the decisions I took.

The last but absolutely not the least, I have to thank my parents. My father, Baba, your gravitas, seriousness, and logical thinking has been source of lots of inspiration for me. I am well-aware of the time period, that financially it was hard for you, but anyhow, you sent me to a good school, which formed my entire future life. I can only mention I am indebted for my entire life to you. My mother, mamani, words are so pale and useless for expressing sacrifices you have made for me. I can only say that I am in awe of your overwhelming love. I am pretty sure I can never, understand how someone can selflessly love another without even having the tiniest expectation. I am certain that I cannot compensate any of those things you both have done for me. But I pray to God to empower me to at least understand, and never forget what you have done for me.

Hamed Ghasemieh

4-Jan-2017

1:33 AM 

CONTENTS

1 INTRODUCTION

1.1 Model, Requirement and Verification .......... 2

1.2 Modelling features of critical infrastructures ....... 3

1.3 The context and approach of the thesis . . . . . . . . 4

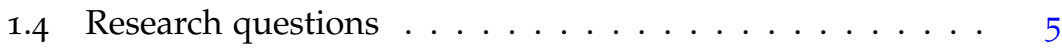

1.5 Thesis outline ................. 8

I MODELLING FORMALISM AND LOGIC II

2 BASICS OF PETRI NETS 13

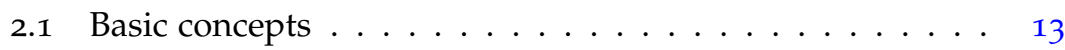

2.1.1 Places, transitions and arcs .......... 13

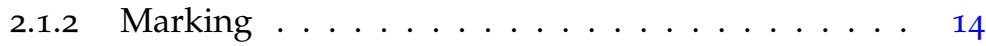

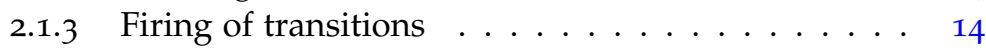

2.1.4 Conflicts and boundedness . . . . . . . . . 15

2.2 Discrete Timed Petri nets . . . . . . . . . . . . 16

2.2.1 Timed transitions . . . . . . . . . . . . 17

2.2.2 Zeno behaviour . . . . . . . . . . . . 18

2.3 Continuous Timed Petri nets . . . . . . . . . . . . . . 19

2.3.1 Token division . . . . . . . . . . . . . . 19

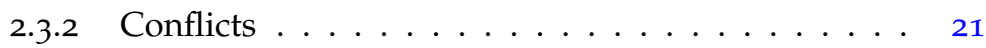

2.4 Related work. . . . . . . . . . . . . 22

3 HYBRID PETRI NETS WITH GENERAL TRANSITIONS 25

3.1 Model Definition . . . . . . . . . . . . . . . 26

3.2 Model Evolution . . . . . . . . . . . . . . . . . 29

3.2.1 Markings, firings, and enabling rules . . . . . 30

3.2.2 Evolution of continuous variables ........ 32

3.2 .3 State of the model ............ 34

3.3 Examples ........................... 34

3.3.1 Reservoir example: basics .............. 34

3.3.2 Control Example: guard arcs . . . . . . . . . . 36

$3 \cdot 3.3$ Overflow place: dynamic transitions . . . . . . . . 38

3.4 Conflict resolution for discrete transitions . . . . . . . . . 39

3.5 Conflict resolution for continuous transitions . . . . . . . 41

3.5.1 Conflict resolution using only priority . . . . . . 43

3.5.2 Conflict resolution using priority and sharing . . . 46

3.6 Related Work . . . . . . . . . . . . 55

4 StATE SPACE AND LOGIC 57

4.1 Stochastic Time Diagrams . . . . . . . . . . . . . . 57

4.1.1 One Stochastic Variable . . . . . . . . . . . . 58

4.1.2 Multiple Stochastic variables: formalization . . . . 61 
4.2 Stochastic Time Logic . . . . . . . . . . . . . . . . . . 64

4.3 Related Work . . . . . . . . . . . . 67

II ANALYSIS AND ALGORITHMS 71

5 EFFICIENT STATE SPACE GENERATION 73

5.1 One Stochastic variable . . . . . . . . . . . . 74

5.1.1 General transition has notfired yet . . . . . . . . 75

5.1.2 General transition has fired . . . . . . . . . . 77

5.2 Multiple General Transitions . . . . . . . . . . . . . . . 82

5.2.1 Preliminaries .................... 82

5.2 .2 Facets and regions ............... 83

5.2.3 Partitioning and Generation ........ . 84

5.2 .4 Finiteness and termination .......... 87

5.2 .5 Complexity ....................... 88

5.2.6 Reservoir Example: revisited . . . . . . . . . . 88

5.3 Related Work . . . . . . . . . . . . . . . 89

6 MEASURE COMPUtATION AND MOdEL CHECKING 93

6.1 Model checking state-based formulas . . . . . . . . . . . 94

6.1.1 One stochastic variable: atomic properties . . . . . 94

6.1.2 Multiple stochastic variable: model checking statebased formula . . . . . . . . . . . . 96

6.1 .3 Case studies . . . . . . . . . . . . . . . 97

6.2 Model checking Until formulas . . . . . . . . . . . . . . 102

6.2.1 Notation ................. 103

6.2.2 Model checking the until operator: preliminaries . 104

6.2.3 Model checking the until operator: algorithm . . . 106

6.2.4 Complexity .................. 110

6.2.5 Case study: Model Checking Until operator . . . . 111

6.3 Related Work . . . . . . . . . . . . . 115

7 APPROXIMATION TECHNIQUES 119

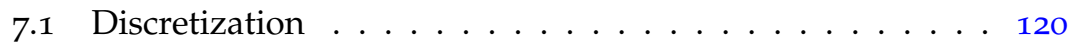

7.1.1 Approach and algorithm .......... 120

7.1.2 Control Example ................ 122

7.2 Probabilistic Time Transitions . . . . . . . . . . . . 125

7.2.1 Notation and definitions . . . . . . . . . . . 126

7.2 .2 State evolution tree . . . . . . . . . 127

7.2.3 Exploring the state evolution .......... 131

7.2.4 Case study . . . . . . . . . . . . . . . . . . 134

$7 \cdot 3$ Notes on the differences of two methods . . . . . . . . 137

7.4 Related work. . . . . . . . . . . . . 139

III REAL-WORLD APPLICATIONS 141

8 ENSCHEDE SEWAGE TREATMENT FACILITY 143

8.1 System and Model . . . . . . . . . . . . . . . 144

8.2 Evaluations ..................... 147 
8.2.1 Scenario A: Heavy rain . . . . . . . . . . . 147

8.2.2 Scenario B: Failure in sand interceptor . . . . . . 150

8.3 Multiple general transitions: Discretization . . . . . . . 152

8.3.1 Feasibility and efficiency ........... 153

8.3.2 Discretization methods ............. 154

8.4 Related Work . . . . . . . . . . . . . 160

9 CONCLUSIONS 161

$\begin{array}{lr}\text { IV APPENDIX } & 167\end{array}$

A ENERgy RESILIENCE MODELLING FOR SMART HOUSES 169

A.1 System and Model . . . . . . . . . . . . . 170

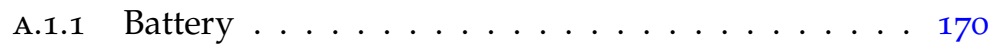

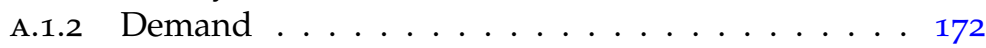

A.1.3 Production.................... 172

A.1.4 Grid and Management Unit . . . . . . . . . 172

A.2 Evaluation .......................... 176

A.2.1 Measures of interest . . . . . . . . . . 176

A.2.2 Parameter choices .............. 177

A.2.3 Survivability results . . . . . . . . . . 177

A.2.4 Computation times . . . . . . . . . . . . 184

A.3 Related work. . . . . . . . . . . . 184

$\begin{array}{ll}\text { BIBLIOGRAPHY } & 187\end{array}$ 



\section{INTRODUCTION}

Many systems around us are naturally hybrid, i.e., they encompass both discrete and continuous dynamic behaviour. In a broad sense, hybrid systems include a combination of interacting continuous flow dynamics (described using differential equations) and discrete event jumps (modelled using state machines or automata). Over the past decades, with the rapid development of information technologies and the growth of computation power, our ability to model and verify such systems has substantially increased.

One of the finest and most important examples of hybrid systems are critical infrastructures. More and more, our society and economy rely on the well-operation of, often hidden, critical infrastructures. These infrastructures, such as the power grid and water and gas distribution networks, play an ever-increasing role in our everyday life. Continuity of supply of services from these assets is essential for people, organizations, and the security and our economy and society $[1,2]$.

Like everything around us, because of advancement of technologies in different aspects, critical infrastructures are also undergoing changes and are becoming more and more complex. Advances in information technology have enabled us to cope with this complexity and provided us with means to rely on them, for our everyday use. However, increase of complexity always comes at the price of vulnerability and increased danger of large scale disruptions. Dependability analysis and the study of cyber and physical vulnerabilities of such infrastructures, are of utmost importance. Moreover, it is of substantial value to know or estimate how quickly such systems can recover to acceptable levels of service after the occurrence of failures, natural disasters (e.g., fire earthquakes) or cyberattacks. This is the focus of study in this thesis.

Let us consider a sewage treatment facility in Enschede as an example of a critical infrastructure. Figure 1.1 depicts a bird's eye view of this facility. Practically any water which has been touched by households or industry is called sewage and needs to be cleaned, in several phases from chemical or physical contamination, before being safely disposed to the environment. This is being done via a network of interconnected tanks, which are being filled and emptied by pumps. An important 


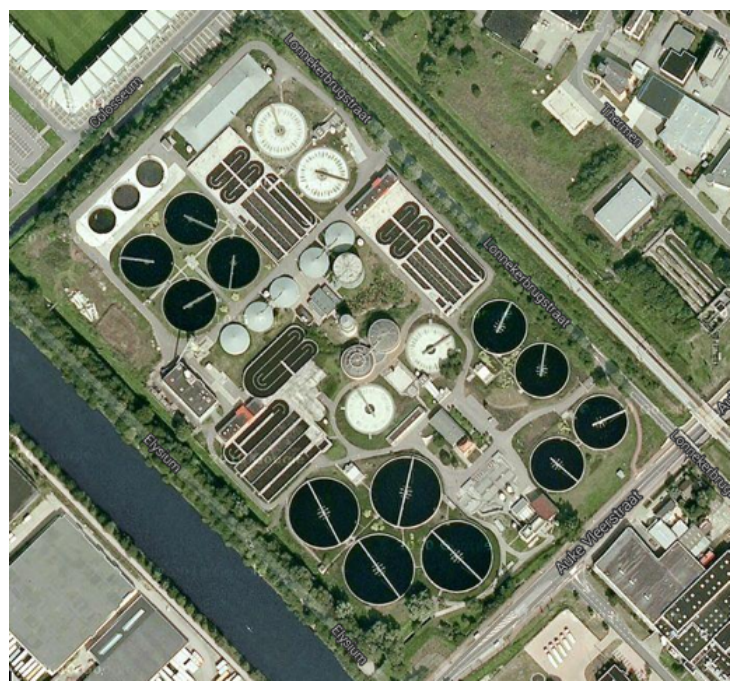

Figure 1.1.: A bird's eye view picture of the sewage treatment facility in Enschede, the Netherlands. The picture is retrieved using Google Maps.

feature of such facilities is that they have a predefined capacity, i.e., the intake of the system cannot exceed a certain value. At the same time, the waste water coming from households or industries is not separated from rain water. Therefore, in case of heavy rain, the streets around the facility may be flooded with sewage, since the system does not allow more intake than the specified amount. This indeed happens from time to time, at least in Enschede, as shown in Figure 1.2, which hinders the life of citizens. Therefore, an analysis to identify bottleneck or vulnerable components in the facility, which may lead to the avoidance or better prediction of service failure in the system, is of substantial value.

\subsection{MODEL, REQUIREMENT AND VERIFICATION}

There are three core elements involved in the verification of hybrid systems, such as critical infrastructures: modelling formalisms, requirement specifications, and verification.

A modelling formalism describes the system under study. This description should be mathematically rigorous and needs to encompass the relevant characteristics of the real world structure. It is important to realize that the results of further analysis (regarding the model) are coherent with the real world application as much as the devised model is. In the next section we discuss the features that the modelling formalism describing critical infrastructures should adhere to. 

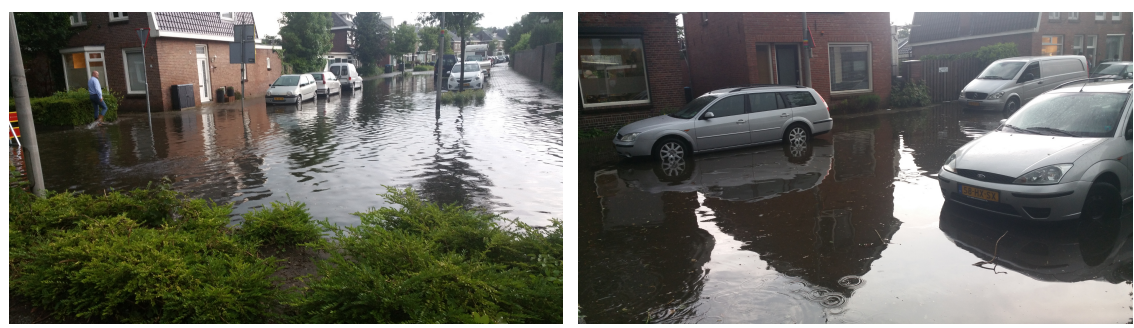

Figure 1.2.: Flooded streets in Ensched. Photographs are courtesy of the author.

The requirements specification formally captures the desired behaviour of the system. One natural way to describe requirement of systems evolving in time is using temporal logics. Intuitively, a temporal logic specifies the expected evolution or behaviour of the system over time [3]. Critical infrastructures are sometimes investigated at a given time, i.e., it is analysed whether a certain requirement is satisfied at a given time of a day. Often more complex requirements, such as so-called survivability needs to be evaluated [4-6]. The survivability of a system is defined as the probability that the system recovers within a predefined amount of time to a predefined level of service. It is mostly evaluated for so-called "Given the Occurrence Of Disaster" (GOOD) models [7]. In such models, as the name suggests, the occurrence of a disaster is assumed to happen at a certain point in time, instead of trying to predict the probability of a disaster using risk assessment. The focus then lies on the effect, the handling and the recovery of the disaster, once it has happened.

Verification is the process of investigating whether the model satisfies or violates a set of given requirements. In a general sense verification approaches can be divided into two main categories: theorem proving and model checking. Theorem proving is usually not a fully automatic process, and is in need of manual manipulation or assistance. On the other hand, model checking is a fully automatic process. Usually the exact verification of requirements using model checking involves the exhaustive search of the model's state space. However, it is not a surprise that this may not be always applicable due to infinite or very large state spaces. Therefore, based on the model at hand and the structure of the state space, many methods have been introduced. We will later discuss model checking techniques related to the content of this dissertation.

\subsection{MODELLING FEATURES OF CRITICAL INFRASTRUCTURES}

As mentioned in the opening of this chapter, critical infrastructure are naturally hybrid, i.e., one needs both discrete and continuous quantities to realistically describe their behaviour. Moreover, in many modern ap- 
plications there is an intrinsic uncertainty, therefore it is important that the devised model provides means for incorporating randomness. This is particularly true for dependability analysis of critical infrastructures, where one must model the occurrence of failures and repairs in a system, which are usually associated with probability distributions. In the following we describe necessary modelling features needed to describe critical infrastructures.

Discrete variables. These are the variables which can take countably many values from a (usually finite) discrete set. Many characteristics of critical infrastructures fall into this category. For instance the binary status of a pump, which may work or not in a sewage treatment facility, or the number of spare parts, or the number of times a switch can be turned on or off.

Continuous variables. These variables can take infinitely many values from a dense set, and usually their evolution can be described via differential equations. For instance, time itself is a continuous variable following a constant differential equation. Another example is the amount of fluid inside tanks in the sewage treatment facility which is being filled or emptied with a constant rate, equal to the difference of input and output rates to the tank, under normal conditions.

Stochastic variables. These variables encompass the lack of knowledge or the intrinsic uncertainty in a system. For instance, in a water treatment facility, a pump can have a failure but the time of this failure may not be determined in advance; or there may be a sudden change of weather which will influence the overall intake to the facility. The occurrence time of such events is usually modelled using random variables, which are associated with probability distributions, specified in the modelling phase.

The values of these variables together define the state of the system. As we will see later, the smart characterization of (groups of) system states plays an important role in efficient verification of the system.

\subsection{THE CONTEXT AND APPROACH OF THE THESIS}

The modelling features described for critical infrastructures in the previous section, place them in the broad class of so-called Stochastic Hybrid Models (SHMs). Over the years, many modelling formalisms have been introduced to describe and evaluate the dynamics of SHMs [8-16]. Each of the approaches extends one of the conventional hybrid models [17-20] with either discrete or continuous probability distributions. These works mainly differ in where the randomness is integrated into the model. One obvious approach is replacing (non)deterministic jumps between system states by probabilistic jumps $[8,11,14,16]$. In this case the stochastic variables are the random variables capturing the occurrence time of these 
jumps. The second approach is to allow randomness in the evolution of continuous variables $[9,10,15]$. More specifically, the ordinary differential equations, governing the evolution of the continuous variables, are replaced by stochastic differential equations.

In this dissertation, in accordance with the modelling features described in the previous section, we undertake the first mentioned approach. The existing models for this approach, from a practical point of view, i.e., both the efficiency of performance, and the class of real-world application that can be modelled, have some limitations. These limitations relate to either the behaviour of the stochastic variables (which is, e.g., limited to only exponential distributions), or the number of continuous variables that can be handled by the model checking of specified requirements. These constraints limit the dependability analysis of critical infrastructures, (which is the main application area that this thesis is aiming for).

This thesis uses hybrid Petri nets [20]. There are several approaches for extending Petri nets (either hybrid or not) with stochastic behaviour [16,21-25]. These modelling formalisms combine discrete and continuous quantities with probabilistic jumps, i.e., random discrete events, hence, allow to model random phenomena in a natural way. Furthermore, Petri nets provide a very high level and easy to understand formalism, which makes it available to a more general audience compared to other formalisms, such as hybrid automata.

In this thesis we consider an extended version of stochastic hybrid Petri nets, in which discrete events can occur with arbitrary probability distribution. Particularly, we extend Hybrid Petri nets with General transitions (HPnG) [26]. The term general transition refers to the arbitrary nature of the probability distribution associated with stochastic variables in this model, which represent the time of occurrence of discrete events. These events correspond to the firing of discrete transitions in Petri nets. Like any other modelling formalism, also HPnGs have their restrictions. For instance, they impose limitation on the evolution of continuous variables to be linear in time. Hence, the change of continuous variables is piecewise constant over time. Because of this limitation, HPnGs form a restricted subclass of SHMs.

In order to express the system requirements, such as safety or survivability properties, we introduce a new logic. The semantics of this logic is defined such that the computation of measures of interest, via a model checking algorithm, is both efficient and effective.

\subsection{RESEARCH QUESTIONS}

As mentioned in the previous section, in this thesis we consider SHMs, in which the randomness is integrated using probabilistic jumps be- 
tween discrete system states. The analysis of these SHMs in general is complicated and in some cases the exact computation of measures of interests is impossible. Hence, one needs to impose restrictions on different aspects of the system in order to make the analysis possible for a specific application area. One option for such restrictions is to reduce the complexity of the model by imposing a limit on the number of continuous variables $[16,24,25,27]$. A more general approach for practically making analysis of stochastic hybrid models possible, is by assuming certain conditions on the stochastic behaviour of the system. For instance, one may assume that all the occurrence times of events in a model follow an exponential distribution; so that one can use the available mathematical tools for analysis of Markov models [16, 24, 27].

One of the main application areas addressed in this thesis, is the dependability analysis of critical infrastructures. From a modelling perspective, the following characteristic occur frequently when analysing critical infrastructures:

(C1) Dependability analysis of critical infrastructures usually involves diverse random behaviour. Therefore, one needs a general modelling formalism which does not restrict the diversity of probability distributions that describe the random occurrence of events.

(C2) Critical infrastructures usually incorporate many interconnected components each of which may be associated with continuous variables, therefore, assuming a (small) upper bound on the number of these variables is not an option.

(C3) In dependability analysis, exact or near exact analysis is of utmost importance. This is because the risk and costs associated with the occurrence of a disaster is too high, that even a very low probability of occurrence of such events should be taken into account.

(C4) In relation to $\mathrm{C}_{1}$, although critical infrastructures contain random events of arbitrary distribution, the number of these events, and therefore the number of stochastic variables is usually fairly limited.

In this thesis we seek to extend the existing hybrid Petri net modelling formalism and analysis methods, to embody the above characteristics. These bring us to the first research question of this thesis.

$\mathcal{Q}_{1}$ How can arbitrary probability distributions that describe the occurrence of discrete events, be incorporated in hybrid Petri nets, and yet be accompanied by efficient and exact techniques? 
In research question $\mathcal{Q}_{1}$, three phrases are emphasized to connect to the three characteristics $C_{1}-C_{3}$, mentioned above, in the order they have appeared. Note that the ambit of the modelling formalism developed in this thesis is not limited to only modelling critical infrastructures. Indeed, any application area that shows the above mentioned characteristics can be analysed with the methods provided in this thesis.

As mentioned earlier, there are several extensions of Petri nets with probabilistic behaviour, however, they all suffer from limitations. Some of these models only allow discrete variables. Among them some use the assumption that all the stochastic variables follow an exponential distribution and benefit from the existing mathematical tools for Markov models [21, 28-31], and some allow arbitrary probability distributions $[22,32,33]$. On the other hand, some models allow a limited number of continuous variables, along with only exponential distributions for stochastic variables $[16,24,26]$. Having said that, one should notice that $\mathcal{Q}_{1}$ is an ambitious approach for stochastic hybrid models in general, and hybrid Petri nets in particular. Therefore, it is important to investigate how complex the system model can be and still be analysed efficiently. As a measure of complexity we consider the number of stochastic and continuous variables in the system. Therefore, it is important to characterize the correspondence of these variables with the modelling components, and discuss the conditions and upper limits (if any) on the number of these variables, such that still efficient and exact analysis is possible. Hence the second research question, can be described as follows, by particularly focusing on characteristics $\mathrm{C}_{2}$ and $\mathrm{C}_{4}$ :

Q2 What are the limitations of the modelling formalism, and analysis methods devised in response to $\mathcal{Q}_{1}$ ? In other words, how far can the complexity of the proposed model, in terms of its numbers of stochastic and continuous variables be pushed, considering efficiency and exactness of the analysis methods?

Having characterized the limits of complexity of the system for exact analysis, we would like to see if it is still possible to provide approximate answers for model checking and computation of measures of interest. More specifically, for certain applications it is still valuable to come up with lower and upper bound approximations for satisfaction probabilities of certain properties. Therefore, we can characterize the third research question as follows: 
$\mathcal{Q}_{3}$ In light of $\mathcal{Q}_{2}$, can we develop algorithms to provide approximate results for more complex systems?

\subsection{THESIS OUTLINE}

This thesis consists of three main parts and an appendix. In the first part, Modelling Formalism and Logic, we introduce and formalize the concepts of the model and the logic for expressing the requirements. The second part, Analysis and Algorithms, is the core of the thesis, which includes the algorithms for the generation of the state space and computation of measures of interest, both exactly and approximately. Finally, the third part, Real-world Applications, together with the appendix, provide an indepth dependability analysis of two separate case studies from different application areas. In the following we provide a brief overview of the contents of each chapter.

\section{Part I Modelling Formalism and Logic}

Chapter 2 presents the required background for Petri nets, including the modelling formalism and semantics. This chapter builds the foundation for later discussions on hybrid Petri nets.

Chapter 3 presents the definition and evolution of HPnGs. In this chapter we formalise all the details of the modelling formalism of HPnGs, and using examples we show their modelling power.

Chapter 4 provides the informal description of the idea of generation of the underlying state space, which is based on the separation of stochastic from deterministic behaviour of HPnGs. As we will see, the number of stochastic variables plays an important role in the shape of the state space. Therefore, we first describe the concepts of state space generation by assuming that a single stochastic variable exists in the system. Subsequently, we expand it to the general case of multiple stochastic variables. In this chapter we also define and formalize the semantics of the logic for expressing the requirements.

\section{Part II Analysis and Algorithm}

Chapter 5 formalizes the algorithm for the generation of the underlying state space. Following the same style in Chapter 4, we first discuss the algorithms for the case of one stochastic variable and later extend it for multiple variables. 
Chapter 6 discusses the algorithms for computing measures of interest. In this chapter we consider two different types of formulas expressed in the logic of Chapter 4. Particularly, we first consider state-based formulas, which express static properties at a given point in time. Second, we investigate temporal properties, i.e., properties about the evolution of the system, using the classic Until operator. We consider these formulas separately because the devised algorithms for their model checking are different.

Chapter 7 tackles the main limitation for the analysis methods for HPnGs, namely, the restriction on the number of stochastic variables. In this chapter we introduce two methods for approximating measures of interest which both are based on the discretization of the support of stochastic variables. The first method is the modification of a previously proposed method in Chapter 6, whereas the second method is a completely new approach, which provides a lower and upper approximation for the measures of interest.

Part III Real-world Applications

Chapter 8 introduces a detailed model of a water and sewage treatment facility in Enschede, using HPnGs. We provide a detailed dependability analysis of this facility, by parametrizing different features. As a result we provide an insight on the vulnerable parts of the system.

Chapter 9 concludes the dissertation, and provides some lines for future work.

\section{Part IV Appendix}

Appendix A considers the second real-world application, energy resilience modelling for smart houses. In this appendix we discuss the best strategies for charging the back-up battery inside a house equipped with solar panels. In other words, we investigate what are the best strategies for balancing the use of the grid and locally produced energy such that, in case of the grid failure, we can continuously provide electrical energy. ${ }^{1}$

Please note that a detailed review of literature and related work is postponed to the end of each chapter. This is done so that related work can be discussed in the proper context of the contribution of this thesis.

I This application example is placed as an appendix to this thesis, since it has been conducted as a collaboration, which has used the modelling formalism and the algorithm developed in this thesis. 

Part I

MODELLING FORMALISM AND LOGIC 



\section{2}

BASICS OF PETRI NETS

The content of this chapter is based on the presentation of the well-known book by David and Alla [34].

In this chapter we will discuss the basic concepts regarding Petri nets. Petri nets form a powerful modelling formalism which are used in wide variety of application areas. The main advantage of Petri nets is that they provide an easy to understand visual description of the system under investigation, hence, can be employed by users of different background.

In this chapter we first introduce the basic concepts and some recurring properties of Petri nets in Section 2.1. After this we discuss two major classes of Petri nets, namely discrete and continuous timed Petri nets, in Sections 2.2, and 2.3, respectively. Finally in Section 2.4 we will review and provide references to the works related to the material of this chapter.

\subsection{BASIC CONCEPTS}

\subsubsection{Places, transitions and arcs}

In the most general sense a Petri net is a directed graph which consists of two types of nodes: places and transitions. These two types of nodes are connected to each other via arcs, such that if one end of an arc is connected to a place the other is always is connected to a transition, and vice versa. In other words a Petri net is a directed bipartite graph, therefore, any considered path is alternating between set of places and transitions. Figure 2.1a, shows a simple Petri net, in which transitions are depicted as rectangles and places with circles.

Definition 2.1 (Petri nets). A Petri net is defined as a tuple $P N=(\mathcal{P}, \mathcal{T}, \mathcal{A})$, in which $\mathcal{P}$, and $\mathcal{T}$ are the finite sets of places, and transitions respectively, and $\mathcal{A} \subseteq(\mathcal{P} \times \mathcal{T}) \cup(\mathcal{T} \times \mathcal{P})$ is the set of arcs connecting places and transitions.

If an arc is directed from a place to a transition (like $P_{1}$ to $T_{1}$ in Figure 2.1a) then the place is called an input to the transition, and if the 


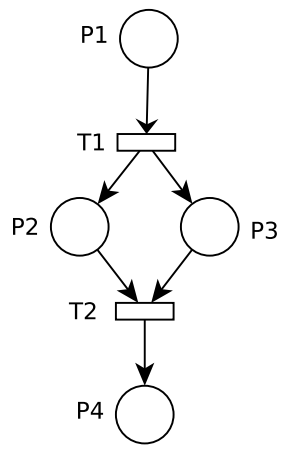

(a) Not marked

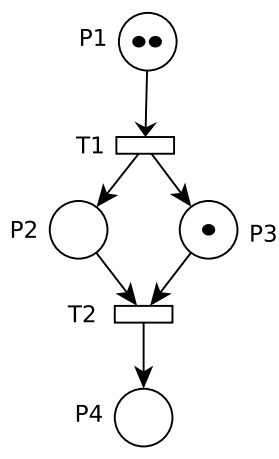

(b) Marked

Figure 2.1.: A primary demonstration of Peteri nets.

direction is from a transition to a place (like $T_{1}$ to $P_{2}$ in Figure 2.1a), the place is called an output for the transition.

\subsubsection{Marking}

Figure 2.1b, depicts a marked Petri net. In this figure places $P_{1}$ and $P_{3}$ are marked since they contain, respectively, two tokens and one token (those small black circles). A Petri net is marked if its places are marked. Marking of a Petri net describes the current state of the Petri net. A marking of a place $P_{i}$ is denoted by $m_{i}$ (or $m\left(P_{i}\right)$ depending on the context), and the current marking of the Petri net is denoted by a vector $\mathbf{m}=\left(m_{1}, \ldots, m_{n}\right)$, in which $n$ is the total number of places, i.e., $n=|\mathcal{P}|$. For instance, the marking of the Petri net in Figure 2.1b, is $\mathbf{m}=(2,0,1,0)$.

A Petri net $\mathbf{P N}$, is usually defined together with its initial marking, denoted by $\mathbf{m}_{0}$, and is presented by the tuple: $\left(\mathbf{P N}, \mathbf{m}_{0}\right)$.

\subsubsection{Firing of transitions}

When a transition fires it imposes a change on the marking of the Petri net, by removing a token from all its input places and adding a token to each of its output places. A transition can fire only if all its input places contain at least a token. So, we say a transition is enabled if all its input places contain at least one token. ${ }^{1}$ As a special case, if a transition does not have any input place, it is always enabled, and may always fire. We call this sort of transitions, sources, for the obvious reason that they produce tokens without dependency on other parts of the network. Figure 2.2 shows the possible firing of a transition in three different setting:

1 We do not consider weights for arcs at the moment. 
in Figure 2.2a and 2.2b transition $T_{1}$ is enabled, whereas in Figure $2.2 \mathrm{C}$ since $P_{1}$ is empty, it is not enabled. As can be seen the firing of $T_{1}$ in both Figures 2.2a and 2.2b will remove a token from places and $P_{1}$ and $P_{2}$, and will add a token to each of the places $P_{3}$ and $P_{4}$.

(a)

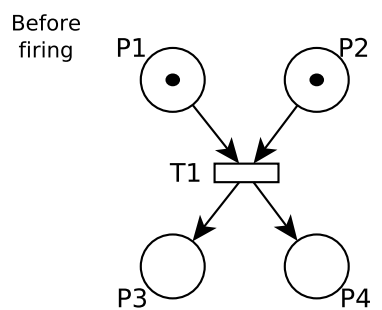

After firing

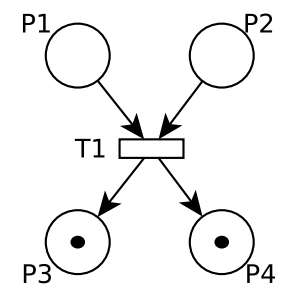

(b)

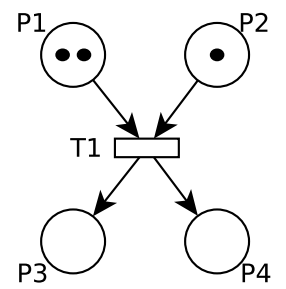

$\sqrt{3}$

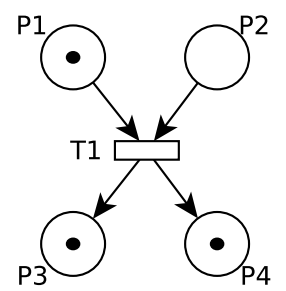

(c)

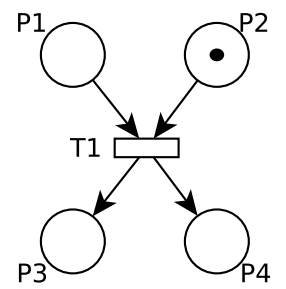

Figure 2.2.: Firing of a transition in three different setting. In (a) and (b) the transition is enabled, whereas in (c) it is not enabled, since place $P_{1}$ is empty.

Definition 2.2 (Reachability). We say that marking $\boldsymbol{m}$ is reachable from marking $\boldsymbol{m}_{0}$ if there is a sequence of firings of transitions that can take the Petri net marking from $m_{0}$ to $m$.

As an example, a firing sequence, hence, a marking sequence in Figure 2.1b is: $(2,0,1,0) \stackrel{T_{1}}{\longrightarrow}(1,1,2,0) \stackrel{T_{2}}{\longrightarrow}(1,0,1,1)$.

One important remark is that when a transition is enabled it does not mean that it will fire immediately. The firing in case of being enabled, is considered merely as a possibility, therefore we only know if the transition fires, what is the next state. We will later formalize this by introducing Timed Petri nets in Section 2.2.

\subsubsection{Conflicts and boundedness}

The concept of conflicts is going to appear frequently in this thesis. It happens when one input place is connected to two or more transitions. 
In this case, depending on which one of the transitions fires first, the next marking of the Petri net may be different. This situation is illustrated in Figure 2.3. Based on how the firing transition is selected among $T_{1}$ and $T_{2}$, the next marking of the Petri net is different. One way to take care of this situation is by assigning priorities to transitions and choosing the one with higher priority in the presence of a conflict. The other possible scenario for resolution of conflicts is by assigning weights to transitions and choosing the the next transition probabilistically based on the preassigned weights. We will elaborate on conflict resolution among discrete transitions in the Chapter 3 .

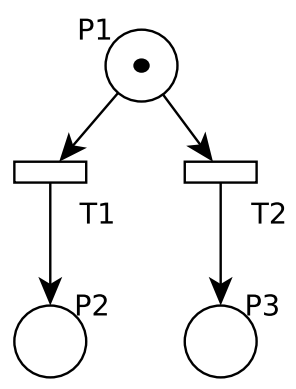

Figure 2.3.: A conflict between transition $T_{1}$ and $T_{2}$.

Another important concept is the concept of boundedness, which requires that the number of tokens in the Petri net is bounded. An infinite number of tokens can occur, for instance, when we have a source transition, i.e., a transition which is not connected to any input place, and therefore can produce infinitely many tokens.

Definition 2.3 (Boundedness [34]). A place is called bounded with respect to the initial marking $m_{0}$, if there exists a non-negative integer number $k$ such that for any marking reachable from $\boldsymbol{m}_{0}$, the number of tokens in that place will not exceed $k$. A Petri net is called bounded with respect to initial marking $m_{0}$ if all the places are bounded with respect to $\boldsymbol{m}_{0}$.

The concept of boundedness is of great importance in this dissertation, as it provides guarantees for correctness and termination of the algorithms to be presented in the next chapters.

\subsection{DISCRETE TIMED PETRI NETS}

In this section we discus how the firing of transitions in a Petri net can be scheduled, i.e., how we can associate time with firing of transitions, and hence describe the evolution of markings. Note that the term discrete, 
refers to the discrete content of places, i.e., each place can contain a discrete number of tokens.

\subsubsection{Timed transitions}

The associated time to the firings of transitions can be both constant or stochastic. In this section we only consider constant timing for transitions; and in chapter 3 we introduce general transitions with arbitrary stochastic behaviour.

Definition 2.4. A discrete timed Petri net is defined as a pair $\left(\boldsymbol{P N}, \boldsymbol{m}_{0}, \phi_{d}\right)$, in which PN is a Petri net as defined in Definition 2.1, $m_{0}$ is the initial marking, and $\phi_{d}: \mathcal{T} \rightarrow \mathbb{R}^{+}$is a function which assigns to each transition $T \in \mathcal{T}$ a firing time $a \in \mathbb{R}^{+}$.

When a transition $T_{i}$ is enabled it does not fire immediately, it waits until time $\phi_{d}\left(T_{i}\right)$ is elapsed and then fires (unless there is a conflict). In order to schedule firings, we assign to each transition $T_{i}$ a clock $c_{i}$, which evolves with rate 1 , only when $T_{i}$ is enabled. When the clock reaches the value of $\phi_{d}\left(T_{i}\right)$, the transition will fire, and the associated clock is reset to zero. It is important to note that, while the clock is evolving it is possible that the transition is disabled by firings of other transitions. In this case we assume that the clock keeps it value, and when the transition is enabled again the clock will resume from its previous value. ${ }^{2}$

When using discrete timed Petri nets the structure given in Figure 2.3, does not necessarily lead to a conflict. Indeed, a conflict may occur when two transitions are sharing an input place and their clocks reach their firing time at the same time. In this case we have a so-called actual conflict.

Figure 2.4 shows an example of a discrete timed Petri net, modelling two machines working sequentially, in which each machine can serve one task at a time. Transition $A$ is modelling a machine working nonstop whenever a token is available in place $P_{1}$ and finishes each task in 3 hours. Each firing of this transition means a task is served. On the other hand, transition $B$ is modelling the second machine. This machine services the tasks which are processed by machine $A$ first, and are placed in $P_{2}$. The service time for machine $B$ is 2 hours, but it also needs another 2 hours of maintenance/preparation after serving each task. This is modelled using transition $M$ with 2 hours delay. Note that transition $B$ is enabled only when $P_{4}$ contains a token, and when $B$ fires $P_{4}$ is emptied. Moreover, a token is placed in $P_{4}$ only after $M$ fires, i.e., when the maintenance has taken place. This ensures the need of maintenance

2 This notion of associating a clock with each transition is different from well-known works like [35], in which transitions firings are coordinated with respect to a globally maintained clock. 


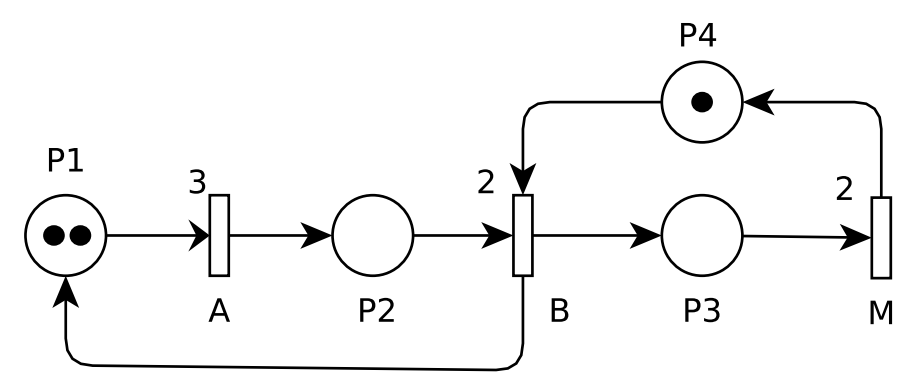

Figure 2.4.: An example of discrete timed Petri nets. The numbers beside transitions are their relative firing times.

for $B$ after servicing each task. As can be seen timed Petri nets provide us with ability to include timing delays in our modelling.

\subsubsection{Zeno behaviour}

When assigning firing times to transitions, we have to pay special attentions to the case when the assigned firing time is zero. We call this type of transitions immediate transitions, as they will fire immediately after being enabled. Immediate transitions may cause an infinite sequence of changes in marking of a Petri net in zero time, since they can fire without any time elapsing. This can be seen in Figure 2.5, in which the infinite sequence of marking: $(1,0) \stackrel{T_{1}}{\rightarrow}(0,1) \stackrel{T_{2}}{\rightarrow}(1,0) \stackrel{T_{1}}{\rightarrow} \cdots$, is taking place in zero time. We call this Zeno behaviour.

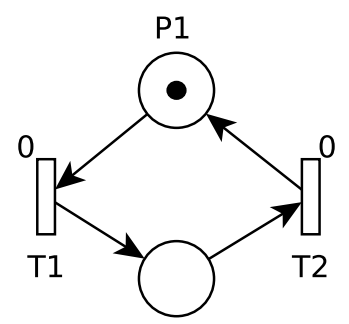

$\mathrm{P} 2$

Figure 2.5.: An example of Petri net with Zeno behaviour. The numbers beside transitions are their relative firing times.

We distinguish two types of markings, based on the type of enabled transitions in the marking: 
Definition 2.5 (Vanishing and tangible markings). A marking is said to be vanishing if there is an enabled immediate transition in it. We call a marking tangible if no immediate transition is enabled in it.

As one can tell, no time is elapsed in a vanishing marking. This is because at the very time a vanishing marking is reached, an immediate transition fires, and the marking is changed. To put it in the new terminology, Zeno behaviour is an infinite sequence of vanishing markings.

If a Petri net is assumed to be bounded (Definition 2.3), the number of possible markings, including vanishing and tangible markings, is finite. ${ }^{3}$ Hence, an infinite sequence of markings, which is the necessary condition for Zeno behaviour, can occur only in cycles, i.e., one marking is going to repeat at least once. Therefore, Zeno behaviour can be detected. This is an important property which we will employ later to provide arguments when discussing the termination of algorithms.

\subsection{CONTINUOUS TIMED PETRI NETS}

It is natural to extend the definition of discrete timed Petri net to continuous timed Petri nets. This is done by allowing the content of places to be continuous values, i.e., real numbers. In this section we limit ourselves to an informal demonstration of continuous Petri nets, as they serve as the underlying structure for hybrid Petri nets in Chapter 3.

\subsubsection{Token division}

Continuous Petri nets can be defined as the limit of discrete Petri nets: each token is divided into $k$ tokens and $k$ is taken to infinity [34]. Consider Figure 2.6a, representing a simple discrete timed Petri net. Transition $T_{1}$ fires with a delay of $d$ time units, and therefore it takes $2 d$ time units to move both tokens from place $P_{1}$ to $P_{2}$. In other words, every $d$ time units a token is moved from $P_{1}$ to $P_{2}$, and we say the token flow rate from $P_{1}$ to $P_{2}$ is $1 / d$ token per time unit, as long as $P_{1}$ is not emptied yet.

Now assume we split each token into two tokens and decrease the delay of transition $T_{1}$ to half, as illustrated in Figure 2.6b. In this modification the time to empty the place $P_{1}$ remains the same, hence, the token flow rate is $2 / d$. If we continue this process, by splitting the tokens into $k$ tokens, and changing the delay of $T_{1}$ to $d / k$, the token flow rate will be $k / d$, and the time for emptying $P_{1}$ still remains the same (cf. Figure 2.6c). This is illustrated in Figure 2.7, by showing the number of tokens in place $P_{1}\left(m_{1}\right)$ versus time, for each Petri nets given in Figure 2.6. Each step in the given step diagrams, corresponds to one firing of $T_{1}$, and removal of one token from $P_{1}$. Therefore two and four firings for Figures 2.6a and 2.6b, respectively.

3 Note that a marking is possible when it is reachable from the initial marking. 


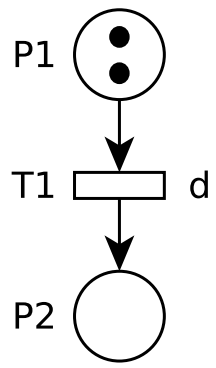

(a)

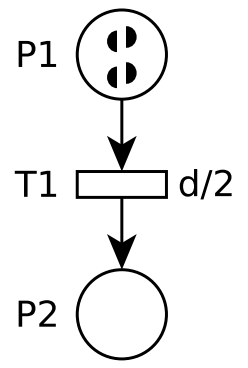

(b)

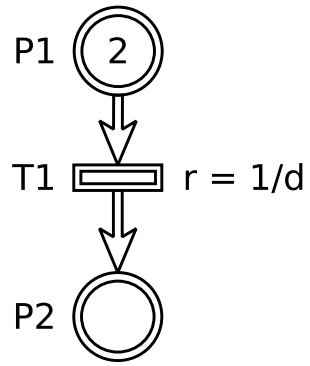

(c)

Figure 2.6.: Continuous Petri nets as the limit of discrete Petri nets [34].

If we let $k$ go to infinity, we can say the marking of place $P_{1}$ is 2 units (instead of tokens), and the flow rate is $r=1 / d$ (units per time). In this setting the time needed for $P_{1}$ to become empty remains the same as previous cases. This limiting case is given in Figure 2.6c, in which discrete places and transitions are replaced with continuous places and transition (drawn with double lined symbols). This limiting case can be seen in Figure 2.7 as a straight line, which is the limits of the previous step diagrams. The slope of this line is the same as the flow rate of transition $T_{1}$.

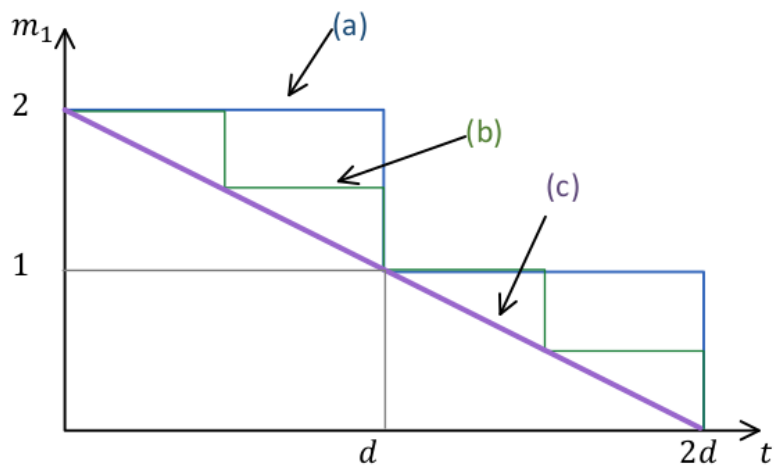

Figure 2.7.: From discrete to continious Petri nets.

As can be seen, in continuous timed Petri nets, transitions are associated with rates or speed instead of firing times or delays. In this case, transitions are assumed to fire continuously, provided they are enabled, i.e., the connected continuous input places are not empty. 


\subsubsection{Conflicts}

As it was discussed we can assign a rate to each continuous transition, however, as we will see in specific situations, a continuous transition cannot fire with this preassigned speed. We call this preassigned value the nominal rate, which can be interpreted as the maximum firing speed. As the naming suggests, a continuous transition cannot always fire with the assigned nominal rate. For instance when a continuous place is empty, obviously it is not possible to remove its content any more, unless it is being filled by by another input continuous transitions at the same time. But in this case the output transition can discharge the place with a rate no more than the rate of the one filling the place. This means that the rate of the connected output continuous transitions should be matched with the rates of input transitions.

Figure 2.8a shows a marking of a Petri net in which there is no conflict and all transitions can work on their nominal rates. However, eventually the place $P_{1}$ is emptied since the overall output rate $(2+3=5)$ is more than the input rate (3.5). Figure 2.8b, shows a conflict situation. As can be seen the continuous place $P_{1}$ is empty but still is being fed by $T_{1}$ (the marking $0^{+}$is used to show this fact). The output transitions, $T_{2}$ and $T_{3}$ cannot fire with their nominal rates, since otherwise we will end up with a negative marking in $P_{1}$, a so-called underflow situation, which is not allowed.

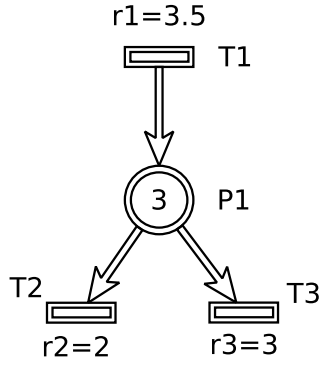

(a) No conflict.

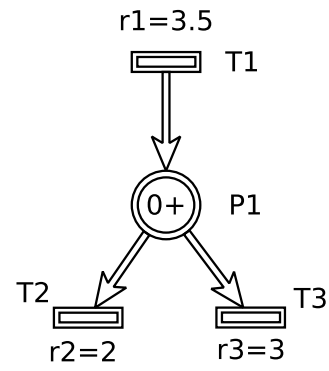

(b) Conflict among $T_{2}$ and $T_{3}$.

Figure 2.8.: Demonstratin of conflicts among transitoins.

Definition 2.6 (Conflict [34]). A timed continuous Petri net is said to have a conflict among transition set $\left\{T_{1}, \ldots, T_{n}\right\}$ for a specific marking, if the firing speed of at least one of these transitions needs to be decreased because of another transition in the set. We call the new adapted rate the actual rate.

Conflicts need to be resolved by adapting the rates of transitions to avoid underflow. This is usually done using linear programming, by in- 
dicating set of linear equations and finding the feasible rates. For instance, in the example of Figure 2.8, we need to have:

$$
r_{2} \leq 2 ; r_{3} \leq 3 ; r_{2}+r_{3}=3.5
$$

The first two equations are describing the fact the the actual rates should be less than or equal to the nominal rates; and the third one is stating the fact that the overall output rate should be matched with the input rate (the rate of $T_{1}$ ).

After finding the feasible solution set for above equations, one has to decide how to distribute the available flow among $T_{2}$ and $T_{3}$. This can be done by assigning priorities and shares to transitions. For example if the priority of $T_{2}$ is higher than that of $T_{3}$, it takes all it can and the rest goes to $T_{3}$, hence the rates would be: $r_{2}=2$ and $r_{3}=1.5$ (since the available flow is 3.5 ). If they have the same priority then the flow is shared among them proportional to their pre-assigned shares. As in the above example, if both $T_{2}$ and $T_{3}$ have the same priority, but different shares, say 1 and 2 , respectively, the available flow (3.5) is distributed among them such that: $r_{2}=3.5 \times \frac{1}{3}$ and $r_{3}=3.5 \times \frac{2}{3}$. Note that share and priorities are preassigned to transitions via the arcs connecting them to the places. We will discuss this in more detail in the next chapter.

We will investigate this so-called rate adaptation process in more detail in Chapter 3, after introducing the formalism of hybrid Petri nets. One can refer to Chapter 5 of [34] for a detailed treatment of conflict resolution for continuous timed Petri nets.

\subsection{RELATED WORK}

According to [36], Carl Petri introduced the concepts and graphical representation of Petri nets in 1939 at the age of 13 for describing chemical processes. However, the formal presentation of Petri nets is given in his $\mathrm{PhD}$ dissertation in 1962 under the original name of Place/Transition nets or P/T nets [37]. He later expanded the network theory in [38].

Associating times with transitions is first introduced in [39]. Following the convention of [34], we have assumed that the firings of transitions are taking place instantly. However, in some other works authors have undertaken another approach: when a transition fires a token is removed from the connected input place(s) and after some time elapsed it is added to output places [40,41]. Here it is also worth mentioning that in some literature, authors have associated times to places instead of transitions [34, 42]. This means that instead of assuming an event occurs after the elapse of time, they assume each place has a determined residence time during which it can hold its tokens.

In general, when transitions are not timed, it is assumed they are firing non-deterministically when they are enabled. In this case it is 
shown that timed Petri nets have the same descriptive power as Turing machines [43,44].

Zeno behaviour has its name from the Greek philosopher Zeno of Elea (five centuries before Christ), who believed that "the being is immobile", i.e., motion does not exist, as it contradicts itself. This problem is known as the Zeno paradox in philosophy [45]. He indeed had an interesting proof for this absurd idea, which now we know is wrong. The reason for this was that in his time mathematics was not developed enough, so he was assuming that infinite sum of numbers is always infinite, which now we know is not true [46].

Continuous Petri nets were first introduced in [47]. Representation of continuous Petri nets as the limiting case of discrete Petri nets, through token "divisions" as we have done here, was first presented in [48]. The idea of conflict resolution and rate adaptation of continuous transitions using linear programming is given in $[49,50]$. 

The content of this chapter is based on:

[26] M. Gribaudo and A. Remke. Hybrid Petri nets with general one-shot transitions for dependability evaluation of fluid critical infrastructures. In 12th International Symposium on High Assurance Systems Engineering, pages 84-93. IEEE, 2010

[51] H. Ghasemieh, A. Remke, B.R. Haverkort, and M Gribaudo. Region-based analysis of hybrid Petri nets with a single general one-shot transition. In Formal Modeling and Analysis of Timed Systems, volume 7595 of LNCS, pages 139-154. Springer, 2012

[34] R. David and H. Alla. Discrete, Continuous, and Hybrid Petri Nets. Springer, 2010.

In this chapter we introduce Hybrid Petri nets with General transitions (HPnG) [26]. HPnGs are intended for modelling systems with stochastic and hybrid characteristics, i.e., for systems in which discrete and continuous variables and also random behaviour are present. An example of such a system is a water treatment facility: the content of tanks is represented by continuous variables, status of pumps (being on or off) is modelled by discrete variables, and the random failure of components or a sudden change of weather are examples of possible stochastic behaviour.

Informally, HPnGs combine discrete and continuous timed Petri nets (both presented in Chapter 2), in which discrete transitions are allowed to have probabilistic behaviours. In other words, discrete transitions, instead of firing at preassigned times after being enabled, are assigned with an arbitrary probability distribution. These distributions determine their firing times. For this reason we also refer to discrete transitions as general transitions.

This chapter is organized as follows. In Section 3.1 we formalize the definition of HPnGs, and introduce a graphical representation for ease of modelling representation. Section 3.2 discusses the semantics and 
evolution of the model, and define the state of the model for further analysis. Section 3.3, provides several examples in order to illustrate how the formalism can be employed for modelling real systems. In Section 3.4 conflict resolution among discrete transitions is discussed. Section 3.5 discusses in detail the resolution of conflict among continuous transitions. This will result in a rate adaptation algorithm. Finally, in Section 3.6 we summarize and provide references to the works related to the contents of this chapter.

\subsection{MODEL DEFINITION}

In this section we define HPnGs. The content of this section is the extended version of the model defined in [26].

An HPnG consists of three main sets of components:

(1) places (discrete and continuous), which model different modes or states of a system,

(2) transitions (discrete and continuous), which allow both probabilistic and deterministic changes between different modes of a system, and

(3) arcs (connecting places and transitions), which determine how the other two component sets are related, i.e., how a transition between different modes (states) of a system can happen.

An important feature of HPnGs are guard arcs. These arcs ensure that a transition is enabled only if the connecting place fulfils certain criteria specified by the guard arc, e.g., if the amount of fluid in a connecting place is more (or less) than a constant in case of a continuous place, or if the number of tokens is more (or less) than a given value, in case of a discrete place.

In certain applications one may need to allow the nominal rate of continuous transitions to depend on the nominal rates of other continuous transitions. We name these continuous transitions as dynamic transitions, and refer to ordinary continuous transitions, those resembling continuous transitions in [34], as static transitions.

Definition 3.1 (HPnG). An HPnG is defined as a tuple $(\mathcal{P}, \mathcal{T}, \mathcal{A}, \Phi)$, where $\mathcal{P}$ is the set of places, $\mathcal{T}$ is the set of transitions, $\mathcal{A}$ are the arcs, and $\Phi$ is a tuple of mappings.

- The set $\mathcal{P}$, is partitioned into a set of discrete places, $\mathcal{P}^{D}$ and a set of continuous places $\mathcal{P}^{C}$.

- The set $\mathcal{T}$ is partitioned into the set of discrete transitions, $\mathcal{T}^{D}$, and the set of continuous transitions $\mathcal{T}^{C}$. The set of continuous transitions, $\mathcal{T}^{\mathrm{C}}$, itself consists of two disjoint sets: static and dynamic transitions, denoted by $\mathcal{T}^{D y}$ and $\mathcal{T}^{S t}$, respectively. 
- The set $\mathcal{A}$ is divided into three subsets:

(i) discrete arcs connecting discrete places and transitions: $\mathcal{A}^{D} \subseteq\left(\mathcal{P}^{D} \times \mathcal{T}^{D}\right) \cup\left(\mathcal{T}^{D} \times \mathcal{P}^{D}\right)$,

(ii) continuous arcs connecting continuous places and transitions: $\mathcal{A}^{C} \subseteq\left(\mathcal{P}^{C} \times \mathcal{T}^{C}\right) \cup\left(\mathcal{T}^{C} \times \mathcal{P}^{C}\right)$, and

(iii) guard arcs connecting discrete transitions to either discrete or continuous places; and connecting continuous transitions only to discrete places: $\mathcal{A}^{G} \subseteq\left(\mathcal{T}^{D} \times\left(\mathcal{P}^{C} \cup \mathcal{P}^{D}\right)\right) \cup\left(\mathcal{T}^{C} \times \mathcal{P}^{D}\right)$.

- The tuple of mappings $\Phi$ is given in Definition 3.2.

As mentioned in the previous chapter, a discrete place $P_{i}^{d} \in \mathcal{P}^{D}$ can contain a non-negative integer number of tokens denoted by $m_{i} \in \mathbb{N}$, whereas a continuous place $P_{j}^{c} \in \mathcal{P}^{C}$ can have a level of non-negative real value, denoted by $x_{j} \in \mathbb{R}_{\geq 0}$.

Note that, static continuous transitions are the ordinary continuous transitions, i.e., they have constant nominal rates. However, the nominal rate of dynamic transitions may depend on the actual rate of any other static transition in the HPnG at hand.

Guard arcs are not allowed between continuous transitions and continuous places. For a discrete transition, guard arcs ensure it is enabled only if the connecting place, either discrete or continuous, fulfills a threshold condition specified on the guard arc (For instance if the amount of fluid in a connecting place is more (or less) than a constant in case of a continuous place, or if the number of tokens is more (or less) than a given value, in case of a discrete place). Moreover, for a continuous transition, guard arcs ensure it is enabled if the connected discrete places contain enough tokens. Note that when the firing of transition does not affect the content of places connected via guard arcs. We will formalize the enabling and firing rules for all types of transitions, in Section 3.2.1.

Definition 3.2. The tuple $\Phi=\left(\phi_{b}^{\mathcal{P}}, \phi_{p}^{\mathcal{T}}, \phi_{S t}^{\mathcal{T}}, \phi_{D y^{\prime}}^{\mathcal{T}} \phi_{G}^{\mathcal{T}}, \phi_{n}^{\mathcal{A}}, \phi_{u}^{\mathcal{A}}, \phi_{s}^{\mathcal{A}}, \phi_{p}^{\mathcal{A}}\right)$ contains nine mappings. These mappings assign properties to the components of the $H P n G$, namely places, transitions, and arcs, as are indicated by superscripts $\mathcal{P}, \mathcal{T}$, and $\mathcal{A}$, respectively.

(1) $\phi_{b}^{\mathcal{P}}: \mathcal{P}^{C} \rightarrow \mathbb{R}^{+} \cup\{\infty\}$ assigns an upper bound to each continuous place.

(2) $\phi_{p}^{\mathcal{T}}: \mathcal{T}^{D} \rightarrow \mathbb{N}$ specifies the priority of each discrete transition to resolve firing conflicts, as is discussed later in Section 3.5.

(3) $\phi_{S t}^{\mathcal{T}}: \mathcal{T}^{\text {St }} \rightarrow \mathbb{R}^{+}$defines the constant nominal flow rate for each static continuous transitions.

(4) Each dynamic continuous transition is associated with a function which determines how its nominal flow rate depends on the actual rates of static 
continuous transitions. This is done via mapping $\phi_{D y}^{\mathcal{T}}: \mathcal{T}^{D y} \rightarrow \mathcal{H}$, in which $\mathcal{H}$ is the space of functions $h: \mathbb{R}^{\left|\mathcal{T}^{S t}\right|} \rightarrow \mathbb{R}^{+}{ }^{1}$

(5) Each discrete transition $T_{i}^{d}$ is associated with a general cumulative distribution function $(C D F), G_{i}$. This is done via the mapping $\phi_{G}^{\mathcal{T}}: \mathcal{T}^{D} \rightarrow \mathcal{C D} \mathcal{F}$, in which $\mathcal{C D F}$ is the space of cumulative distribution functions. We usually assume that for each $G_{i}$ the probability density function (PDF) exists, and we denote it by $g_{i}$.

(6) $\phi_{n}^{\mathcal{A}}: \mathcal{A}^{D} \rightarrow \mathbb{N}$, assigns an integer to each discrete input or output arc, which specifies the number of tokens taken from, or added to, the corresponding place upon the firing of the connected transition.

(7) $\phi_{u}^{\mathcal{A}}: \mathcal{A}^{G} \rightarrow\{(\triangleright, \mathbb{R})\}$, with $\triangleright=\{\geq,<\}$ assigns a comparison operator and a real number to each guard arc. ${ }^{2}$

(8) $\phi_{s}^{\mathcal{A}}: \mathcal{A}^{C} \rightarrow \mathbb{R}^{+}$and $\phi_{p}^{\mathcal{A}}: \mathcal{A}^{C} \rightarrow \mathbb{N}$ specify the share and the priority of the connected continuous transition. These are later used for modifying the rates of continuous transitions in case of conflicts, and avoiding over-and under-flow in continuous places. We elaborate on this in Section 3.5.

The above definition of discrete transitions, given in item (5), is general enough to encompass two well-known and widely-used transition types: immediate transitions, which fire immediately after being enabled, and (deterministic) timed transitions, which fire at a given specific time after being enabled. Both of these transition types are associated with a PDF which accumulates its entire mass on a specific point (i.e., the Dirac function). For the immediate transitions this point is 0 and for the deterministic timed transitions it is an arbitrary given time point. From now on we use the term general transitions to refer to discrete transitions with general probability distributions, excluding Dirac function. Therefore, for practical purposes, immediate and deterministic timed transitions are treated as separate entities. For later use, we denote the sets of immediate, deterministic timed, and general transitions by $\mathcal{T}^{I}, \mathcal{T}^{T}$, and $\mathcal{T}^{G}$, respectively. Trivially we have $\mathcal{T}^{I} \cup \mathcal{T}^{T} \cup \mathcal{T}^{G}=\mathcal{T}^{D}$. Because a deterministic(ally) timed transition fires at a specific time we define the function $\Delta: \mathcal{T}^{D} \backslash \mathcal{T}^{G} \rightarrow \mathbb{R}$, which returns the firing time of non-general discrete transitions. Therefore, if $T \in \mathcal{T}^{D} \backslash \mathcal{T}^{G}$, then we know $\phi_{G}^{\mathcal{T}}(T)=U_{d}(s)$, which is a unit step function at $d \geq 0{ }^{3}$, hence we have:

$$
\Delta(T)=\min _{x \in \mathbb{R}}\left\{x: \phi_{G}^{\mathcal{T}}(T)(x)>0\right\}=d .
$$

1 We do not need to impose any restriction on the function space $\mathcal{H}$, however, in this thesis we only consider the space of linear functions.

2 Note that in order to define several comparison conditions one can place several guard arcs with different comparison operators and threshold numbers between places and transitions.

3 The unit step function is defined as: $U_{d}(s)= \begin{cases}0 & s<d \\ 1 & s \geq d\end{cases}$ 


\section{Graphical representation}

The graphical representation for HPnGs is similar to the presentation in Chapter 2. The primitives of HPnGs are shown in Figure 3.1. A discrete place is graphically represented by a single circle and a fluid place is represented by two concentric circles. A general discrete transition is drawn as an empty rectangle, a continuous static transition is shown as an empty rectangle with double lines, a continuous dynamic transition is shown by a double lined solid rectangle. As we are using immediate and deterministic transitions extensively, we assign to them specific symbols. A deterministic transition is drawn as a grey rectangle, and an immediate transition as a thin black bar. The discrete input and output arcs are drawn as single arrows and fluid input and output arcs are represented by double lines. Guard arcs are drawn with two triangular arrowheads. Section 3.3 provides several examples using this graphical representation.

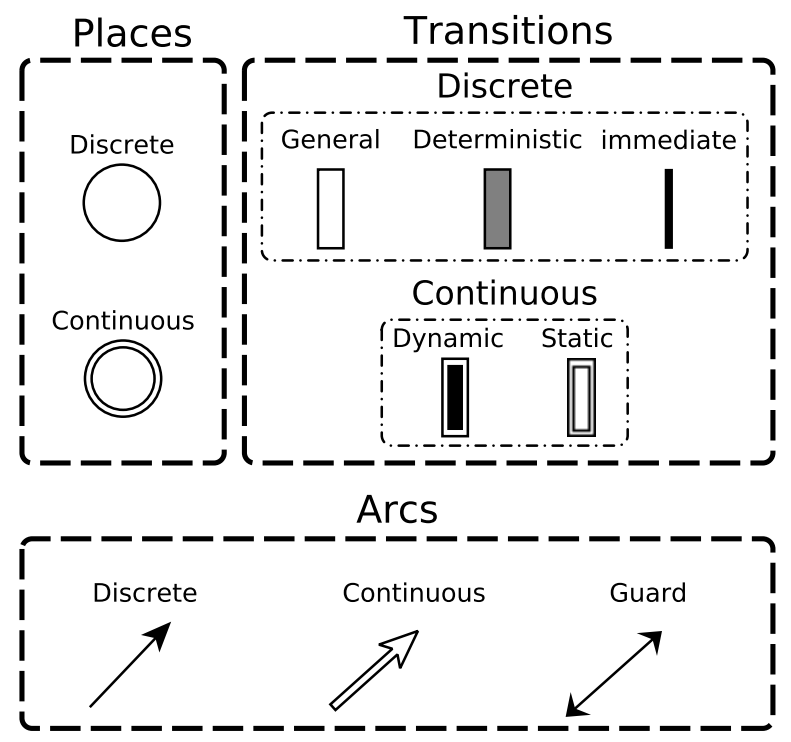

Figure 3.1.: Graphical representation of primitives of HPnG.

\subsection{MODEL EVOLUTION}

In this section we discuss the semantics of the model by defining the marking of HPnG and formalizing the enabling and firing rules of transitions in Subsection 3.2.1. We will also consider how the continuous 
variables evolve in time in Subsection 3.2.2. Finally, in Subsection 3.2.3, we will introduce the state space representation of HPnGs which will be the basis for the analysis methods in this dissertation.

\subsubsection{Markings, firings, and enabling rules}

\section{HPnG marking}

Markings of an HPnG, i.e., content of its places, are collected into two vectors, the discrete marking $\mathbf{m}=\left(m_{1}, \ldots, m_{\left|\mathcal{P}^{D}\right|}\right)$ and the continuous marking $\mathbf{x}=\left(x_{1}, \ldots, x_{|\mathcal{P} C|}\right)$. We refer to the vector $(\mathbf{m}, \mathbf{x})$ as the marking of the HPnG. For a discrete place $P_{i}^{d} \in \mathcal{T}^{D}$, we may use the notation $m\left(P_{i}^{d}\right)$ and $m_{i}$, interchangeably depending on the context (the same goes for continuous places and notation $x\left(P_{i}^{c}\right)$ and $\left.x_{i}\right)$. The initial marking is composed of a discrete part $\mathbf{m}_{0}$ that describes the initial amount of tokens in all discrete places and a continuous part $\mathbf{x}_{0}$ that describes the initial amount of fluid in all continuous places.

\section{Enabling rules}

In order to characterize the firing and enabling rules we need to define the sets of input and output places for transitions:

$$
\begin{aligned}
& \mathcal{I}_{T}(T)=\left\{P \in \mathcal{P}:\langle P, T\rangle \in \mathcal{A}^{C} \cup \mathcal{A}^{D}\right\} \text { (Input places), } \\
& \mathcal{O}_{T}(T)=\left\{P \in \mathcal{P}:\langle T, P\rangle \in \mathcal{A}^{C} \cup \mathcal{A}^{D}\right\} \text { (Output places). }
\end{aligned}
$$

Note that if $T$ is a continuous transition, $\mathcal{I}_{T}(T)$ and $\mathcal{O}_{T}(T)$ contain only continuous places; because only continuous places are allowed to be connected to continuous transitions. The same holds for discrete transitions, i.e., when $T$ is a discrete, $\mathcal{I}_{T}(T)$ and $\mathcal{O}_{T}(T)$ contain only discrete places. This is because no arc is allowed between discrete and continuous components of the HPnG, except for guard arcs, and in the above definition $\mathcal{A}^{G}$ is not included.

The sets of input and output transitions with respect to places are defined as:

$$
\begin{aligned}
& \mathcal{I}_{P}(P)=\left\{T \in \mathcal{T}:\langle T, P\rangle \in \mathcal{A}^{C} \cup \mathcal{A}^{D}\right\} \quad \text { (Input transitions) } \\
& \mathcal{O}_{P}(P)=\left\{T \in \mathcal{T}:\langle P, T\rangle \in \mathcal{A}^{C} \cup \mathcal{A}^{D}\right\} \text { (Output transitions) }
\end{aligned}
$$

Moreover, the set of places connected to a transition via guard arcs is defined as:

$$
\mathcal{G}_{T}(T)=\left\{T \in \mathcal{T}:\langle T, P\rangle \in \mathcal{A}^{G}\right\} .
$$

Now we can characterize when a transition is enabled. 
Definition 3.3. A discrete transition $T^{d} \in \mathcal{T}^{D}$ is said to be enabled if the following three conditions hold:

1. Continuous guard arcs:

$$
\forall P^{c} \in \mathcal{P}^{C} \cap \mathcal{G}\left(T_{d}\right),(\triangleright, q)=\phi_{u}^{\mathcal{A}}\left(\left\langle T^{d}, P^{c}\right\rangle\right): x\left(P^{c}\right) \triangleright q
$$

2. Discrete guard arcs:

$$
\forall P^{d} \in \mathcal{P}^{D} \cap \mathcal{G}\left(T_{d}\right),(\triangleright, q)=\phi_{u}^{\mathcal{A}}\left(\left\langle T^{d}, P^{d}\right\rangle\right): m\left(P^{d}\right) \triangleright q .
$$

3. Connected discrete input places:

$$
\forall P^{d} \in \mathcal{I}\left(T_{d}\right): m\left(P^{c}\right) \geq \phi_{n}^{\mathcal{A}}\left(\left\langle P^{d}, T^{d}\right\rangle\right) .
$$

The first two conditions state that all the connected places (discrete or continuous) should satisfy the threshold of the connecting guard arc, and the third condition checks whether each of the connected discrete input places contains at least the number of tokens specified on the connecting arcs.

Definition 3.4. A continuous transition $T^{c} \in \mathcal{T}^{C}$ is enabled if the following two conditions hold:

1. Discrete guard arcs:

$$
\forall P^{d} \in \mathcal{P}^{D} \cap \mathcal{G}\left(T^{c}\right),(\triangleright, q)=\phi_{u}^{\mathcal{A}}\left(\left\langle T^{c}, P^{d}\right\rangle\right): m\left(P^{d}\right) \triangleright q .
$$

2. Connected continuous input places:

$$
\forall P^{c} \in \mathcal{I}\left(T^{c}\right): x\left(P^{c}\right)>0 .
$$

In the above definition, as continuous transitions are allowed to be only connected to discrete places via guard arcs, we only have one condition regarding guard arcs (the first condition). The second condition states that a continuous transition is enabled only if connected continuous places have positive content, to avoid underflow. Note that, the second condition is a sufficient condition. For instance, if a connected place is empty, but being fed from other transitions, the transition can still be enabled, as discussed briefly in Section 2.3.2.

Firing rules and evolution of discrete variables

Whenever a discrete transition fires the discrete marking of the HPnG, i.e., vector $\mathbf{m}$, changes and the continuous marking $\mathbf{x}$ remains untouched. The firing of a discrete transition $T^{d} \in \mathcal{T}^{D}$ updates the current discrete marking $\mathbf{m}$ as follows:

$$
\begin{aligned}
& \text { (1) } \forall P^{d} \in \mathcal{I}_{T}\left(T^{d}\right): m\left(P^{d}\right) \leftarrow m\left(P^{d}\right)-\phi_{n}^{\mathcal{A}}\left(\left\langle P^{d}, T^{d}\right\rangle\right), \\
& \text { (2) } \forall P^{d} \in \mathcal{O}_{T}\left(T^{d}\right): m\left(P^{d}\right) \leftarrow m\left(P^{d}\right)+\phi_{n}^{\mathcal{A}}\left(\left\langle T^{d}, P^{d}\right\rangle\right) .
\end{aligned}
$$


In update rule (1), the number of tokens specified on the connecting arcs is removed from all input places, and in update rule (2), the number of tokens, specified on the connecting arcs, is added to all output places.

\subsubsection{Evolution of continuous variables}

We have two types of continuous variables present in HPnGs: clocks associated with discrete transitions, and the content of continuous places. In the following we characterize the evolution of these continuous variables over time.

\section{Stochastic variables and clocks}

Discrete transitions in HPnGs, like the ones in Timed Petri nets (Section 2.2), are associated with clocks. These clocks are evolving with rate 1 whenever the transition is enabled. More specifically, let $c_{i}$ be the clock associated with $T_{i}^{d} \in \mathcal{T}^{D}$. Whenever this transition is enabled the change of $c_{i}$ over time is characterized by the differential equation $d c_{i} / d t=1$, and whenever it is not enabled, with $d c_{i} / d t=0$. We refer to the rate of change for a clock (which is either 0 or 1 ) as its drift. When a transition is disabled its clock does not evolve, but the clock value is preserved until the transition is enabled again. Clocks are only reset when the corresponding discrete transition fires.

For a discrete transition, the firing time is specified by its associated CDF. Therefore, the firing time of each discrete transition $T_{i}^{d}$ is specified by a random variable, denoted by $S_{i}$. This random variable is distributed according to the CDF, $G_{i}: \mathcal{S}_{i} \rightarrow[0,1]$, in which $\mathcal{S}_{i}$ is the support of the random variable.

When the discrete transition $T_{i}^{d}$ is enabled for the first time, a value $s_{i}$ is chosen for $S_{i}$ based on the CDF, $G_{i}$. From now on the transition is treated as an ordinary timed transition with firing time $s_{i}$. Hence, when its clock $c_{i}$ reaches the time $s_{i}$, the transition fires, and the clock is reset to zero. Note that $s_{i}$ describes only one possible evolution of the system. We refer to random variables $S_{i}$ as stochastic variables, to emphasis the stochastic behaviour of HPnGs.

As mentioned earlier, in Section 3.1, among the discrete transitions we distinguish general transitions from immediate and deterministic transitions. The latter two do not introduce stochastic variables into the analysis, whereas each firing of a general transition will add one stochastic variable to the system. In order to be able to analyse HPnGs, as we will see later, we need to assume we know how many times a general transition can fire prior to the analysis of the system, hence, we know how many stochastic variables are present. This can be ensured by connecting the general transitions to an isolated discrete input place, i.e., a place that is not connected to any other transition but the specified 
general transition. Therefore, the number of tokens in this discrete place determines the number of times the general transition can fire (at most). Figure 3.2 illustrates a general transition which can fire only two times. This is is ensured by connecting it to the isolated input place $P$ which is initialized with only two tokens.

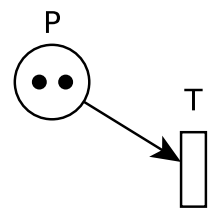

Figure 3.2.: A general transition which can fire twice.

\section{Continuous variables and drifts}

The evolution of the content of a continuous place depends on the connected continuous transitions. We define the actual in-flow and out-flow to a continuous place as follows:

Definition 3.5 (Actual in- and out-flows). The actual in-flow and out-flow to a continuous place $P^{c}$ is defined as the sum of actual rates of input and output continuous transitions:

$$
\begin{aligned}
f_{\text {in }}\left(P^{c}\right) & =\sum_{T^{c} \in \mathcal{I}_{P}\left(P^{c}\right)} r\left(T^{\mathcal{C}}\right), \\
f_{\text {out }}\left(P^{c}\right) & =\sum_{T^{c} \in \mathcal{O}_{P}\left(P^{c}\right)} r\left(T^{c}\right),
\end{aligned}
$$

where $r\left(T^{c}\right)$ is the actual rate of $T^{c}$, as defined in Definition 2.6.

Note that depending on whether $T^{c}$ is a static or a dynamic transition, we have $r\left(T^{\mathcal{C}}\right) \leq \phi_{S t}^{\mathcal{T}}\left(T^{\mathcal{C}}\right)$ or $r\left(T^{\mathcal{C}}\right) \leq \phi_{D y}^{\mathcal{T}}\left(T^{\mathcal{C}}\right)$, respectively. Finally, the drift of the continuous place is defined in the following:

Definition 3.6 (Drift). The drift of the continuous place $P^{c}$ is defined as the difference between its actual in- and out-flow:

$$
d\left(P^{c}\right)=f_{\text {in }}\left(P^{c}\right)-f_{\text {out }}\left(P^{c}\right)
$$

Note that drift can be a positive or a negative real number, meaning the place is being filled or emptied, respectively. Moreover, we have:

$$
\frac{d x\left(P^{c}\right)}{d t}=d\left(P^{c}\right)
$$


in which $x\left(P^{c}\right)$ is the content of place $P^{c}$. Therefore, $d\left(P^{c}\right)$ can also be seen as the change, i.e., derivative of content of place $P^{c}$ over time.

This formalization shows an important characteristic of HPnGs:

Corollary 3.1. At each point in time the evolution of continuous variables (both clocks and content of continuous places) are always characterized by a constant differential equation, i.e., if $x$ is a continuous variable we have:

$$
\frac{d x}{d t}=C \in \mathbb{R} .
$$

Note that, if $x$ is a clock, then $C=1$. This characteristic is later employed for developing algorithms for analysing HPnGs.

\subsubsection{State of the model}

The overall state of an HPnG is defined by $\Gamma=(\mathbf{m}, \mathbf{x}, \mathbf{c}, \mathbf{d}, \mathbf{g})$, where the marking vectors $\mathbf{m}$ and $\mathbf{x}$ were introduced earlier. The vector $\mathbf{c}=$ $\left(c_{1}, \ldots, c_{|\mathcal{T} D|}\right)$ contains clocks $c_{i}$ for each discrete transition $T_{i}^{d} \in \mathcal{T}^{D}$. Vector $\mathbf{d}=\left(d_{1}, \ldots, d_{\left|\mathcal{P}^{C}\right|+\left|\mathcal{T}^{D}\right|}\right)$ indicates the drift of all continuous variables, namely clocks and the content of continuous places, as discussed in the previous section. Finally, the vector $\mathbf{g} \in \mathbb{N}^{\left|\mathcal{T}^{G}\right|}$ indicates the number of times that each general transition has already fired. Hence, the sum of the elements of $\mathbf{g}$, is equal to the total number of present stochastic variables in the system. We denote the initial state of an HPnG by $\Gamma_{0}=\left(\mathbf{m}_{0}, \mathbf{x}_{0}, \mathbf{0}^{\left|\mathcal{T}^{D}\right|}, \mathbf{d}_{0}, \mathbf{0}^{\left|\mathcal{T}^{G}\right|}\right)$, where $\mathbf{0}^{m}$, is the vector with $m$ zero elements.

\section{$3 \cdot 3$ EXAMPLES}

In this section we provide several examples that illustrate how HPnGs can be used to model real systems. In the first example, Section 3.3.1, we demonstrate the basics of the modelling concept. In the second one, given in Section 3.3.2, we show how guard arcs can be employed to control the content of a continuous place between certain amounts, and in the third example, in Section 3.3.3, we illustrate how dynamic transitions can be used to make more complex modelling components targeting real applications.

\subsubsection{Reservoir example: basics}

Here we provide a simple example taken from [26]. Figure $3 \cdot 3$ shows an HPnG model of a reservoir that is filled by a pump and drained due to a demand.

The set of places is $\mathcal{P}=\left\{P_{m}, P_{p}, P_{d}\right\}$, in which $P_{m}$ is continuous (modelling the reservoir) and the other places are discrete. The reservoir $P_{m}$ 
can contain at most 10 units of fluid: $\phi_{b}^{\mathcal{P}}\left(P_{m}\right)=10$. For the set of transitions we have, $\mathcal{T}=\left\{T_{p}, T_{d}, T_{f}, T_{a f}\right\}$, in which $T_{p}$ and $T_{d}$ are static continuous transitions with nominal rates $\phi_{S t}^{\mathcal{T}}\left(T_{p}\right)=2$ and $\phi_{S t}^{\mathcal{T}}\left(T_{d}\right)=1$, which model production and demand pumps. $T_{f}$ is the generally distributed transition with $\operatorname{CDF} \phi_{G}^{\mathcal{T}}\left(T_{f}\right)=G(s)$, and $T_{a f}$ is the deterministic timed transition firing in 5 units of time, hence $\phi_{G}^{\mathcal{T}}\left(T_{a f}\right)=U_{5}(s)$, where $U_{5}(s)$ is the unit step function at 5 .

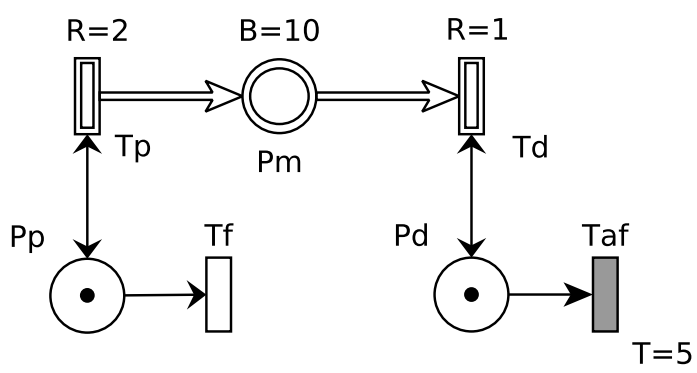

Figure 3.3.: Reservoir model.

Transitions $T_{p}$ and $T_{d}$ continuously fire as long as the discrete places $P_{p}$ and $P_{d}$ contain a token, respectively. This is ensured using the guard arcs $\left\langle T_{p}, P_{p}\right\rangle$ and $\left\langle T_{d}, P_{d}\right\rangle$, such that $\phi_{u}^{\mathcal{A}}\left(\left\langle T_{p}, P_{p}\right\rangle\right)=\phi_{u}^{\mathcal{A}}\left(\left\langle T_{d}, P_{d}\right\rangle\right)=(\geq, 1)$. Note that when the guard arcs connect discrete places and transitions, and no threshold is specified on the graphical representation, we always assume the condition $(\geq, 1)$, i.e., when there is a token in discrete place, the corresponding transition is enabled (given that other related conditions are satisfied).

For the discrete output arcs $\left\langle P_{p}, T_{f}\right\rangle$ and $\left\langle P_{d}, T_{a f}\right\rangle$ the weights are 1, i.e., $\phi_{n}^{\mathcal{A}}\left(\left\langle P_{p}, T_{f}\right\rangle\right)=\phi_{n}^{\mathcal{A}}\left(\left\langle P_{d}, T_{a f}\right\rangle\right)=1$. Therefore, when $T_{f}$ and $T_{a f}$ fire, they remove one token from their connected discrete place, hence, disable $T_{p}$ or $T_{d}$. This can be seen as a deterministic stop at the demand pump, $T_{d}$, and a random failure at the production pump, $T_{p}$.

Figure 3.4 is illustrating two possible evolution of content of continuous place $P_{m}$, assuming that initially the content is 0 . In Figure 3.4a, it assumed that the general transition $T_{f}$ will never fire. Until time $t=5$, the drift of $P_{m}$ is +1 , but at $t=5$ deterministic transition $T_{a f}$ fires, and the drift changes to +2 . Therefore, at time $t=7.5$ the place $P_{m}$ reaches its upper boundary, 10. In Figure $3.4 \mathrm{~b}$, it is assumed that the general transition will fire at time 6 , hence, at this time the drift of $P_{m}$ changes from +2 to -1 . At this time $P_{m}$ contains 7 units of fluid, therefore it reaches its lower boundary 0 , in 7 units of time, i.e., at $t=13$.

Note that Figures 3.4a and 3.4b, are only two possible evolutions of the reservoir example, based on the conditioning on the firing of the general 


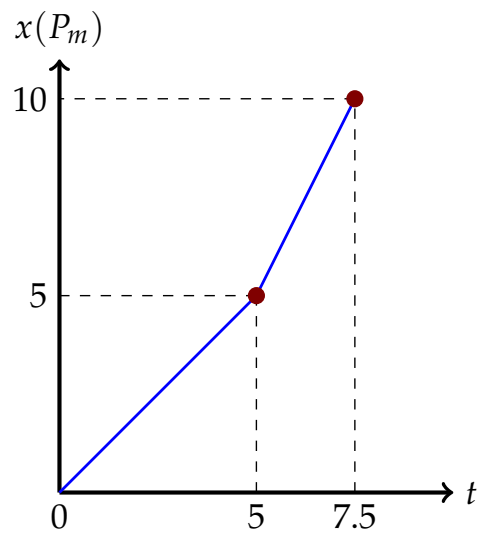

(a) No stochastic behaviour.

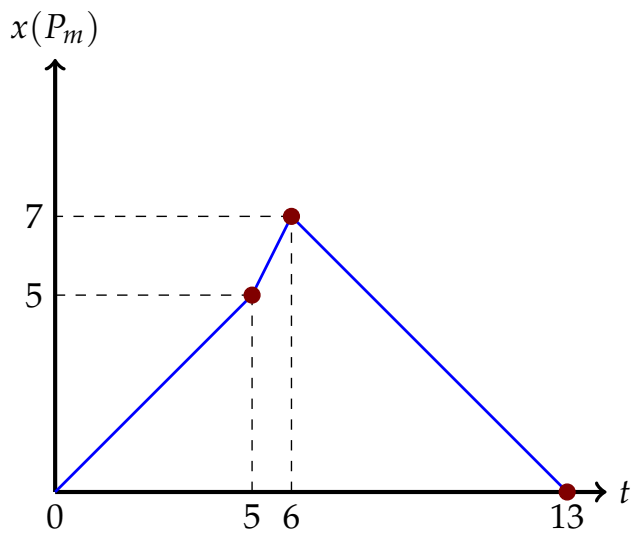

(b) General transition firing at 6 .

Figure 3.4.: Two possible realization for evolution of the content of continuous place $P_{m}$ over time.

transition $T_{f}$. The probabilities of occurrence of each of these evolutions can be determined based on the probability distribution assigned to $T_{f}$.

\subsubsection{Control Example: guard arcs}

In this section we discuss a modification of the reservoir example, where the amount of fluid in the reservoir is supposed to stay between certain bounds. This can be modelled using guard arcs between continuous places and discrete transitions.

As illustrated in Figure 3.5, a tank denoted $P_{m}$ with the capacity of 11 litres, is connected to two pumps. Either, the producer pump $T_{p}$ fills the tank with rate 1 liter/minute, or the consumer pump $T_{d}$ takes out the fluid with rate 2 litre/minute. For control purposes, the amount of fluid in the reservoir needs to be between 1 and 10 avoiding both underflow and overflow. Due to the design the two pumps $T_{p}$ and $T_{d}$ cannot be working at the same time. This is ensured through the discrete places $P_{p}$ and $P_{d}$, since they hold together only one token at each time.

Two switches can turn the pumps on and off, both with a delay of 2 minutes, which is modelled by two deterministically timed transitions. Transition $T_{a}$, with firing time of 2 is connected to the reservoir via a guard arc with condition $(\geq, 8)$. Hence, when the amount of fluid is greater or equal to 8 , it will be enabled and after 2 minutes it will fire. As a result the pump $T_{p}$ becomes disabled and pump $T_{d}$ becomes enabled. Also, transition $T_{b}$, with firing time of 2 , is connected to the reservoir via 
a guard arc with condition $(<, 5)$, so when the amount of fluid is smaller than 5 , the transition will be enabled and fires after two minutes.

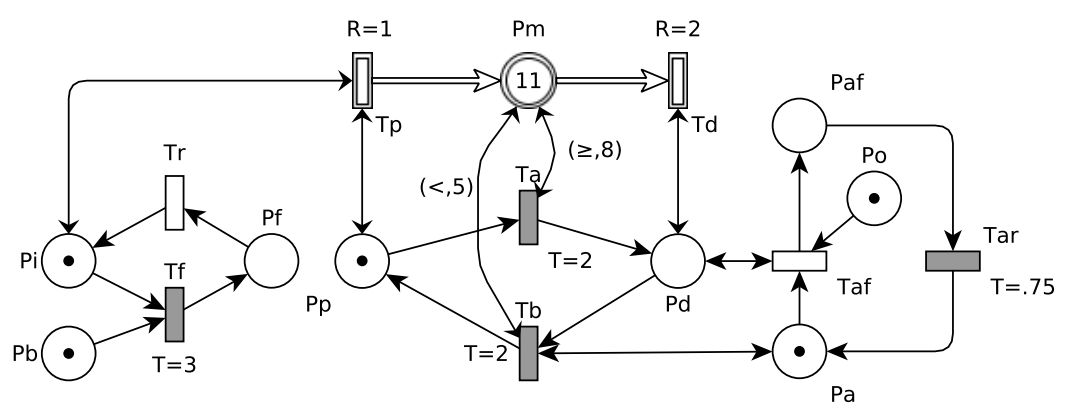

Figure 3.5.: A simple control example. The amount of fluid in the reservoir $P_{m}$ is supposed to remain between 1 and 10.

Figure 3.6 shows the realization of the content of continuous place $P_{m}$ for the case that none of the general transitions has fired. At the beginning, $P_{m}$ is being filled with rate +1 . Once it reaches 8 litres of fluid, the transition $T_{a}$ is enabled, and fires after 2 minutes. This changes the drift of $P_{m}$ to -2 . These two events are indicated with red circles in Figure 3.6. After this, $P_{m}$ is being emptied until it reaches 5 liters of fluid, which causes the transition $T_{b}$ to be enabled. $T_{b}$ fires after 2 minutes, and causes the drift to change back to +1 . These two events are shown by red squares on the figure (at times $t=12.5$ and 13.5). This behaviour repeats periodically from now on. As can be seen, the content of $P_{m}$ always remains between 3 and 10 .

The producer pump may fail at different time points $\alpha$, which is modelled by the deterministic timed transition $T_{f}$, with deterministic firing time parametrized with $\alpha$ (in Figure $3 \cdot 5, \alpha$ is set to 3 ). Whenever this transition fires, the general transition $T_{r}$ becomes enabled. This transition models the repair procedure, which is distributed according to any given probability distribution. Note that the arc between the input place $P_{b}$ and transition $T_{f}$ ensures that $T_{f}$ fires only once, hence, only one failure is possible.

Additionally, we may have another stochastic failure at switch $T_{b}$ that is responsible for turning the demand off and the input pump on, when there is less than 5 litres of water in the reservoir. This possible failure is represented by the general transition $T_{a f}$ and is only enabled when there is a token in place $P_{d}$, i.e., when the demand pump is working. This models, for example, a valve that is stuck open. In the model this failure is followed by a deterministic repair after three quarters of a minute (45 seconds), and transition $T_{b}$ is enabled again. 


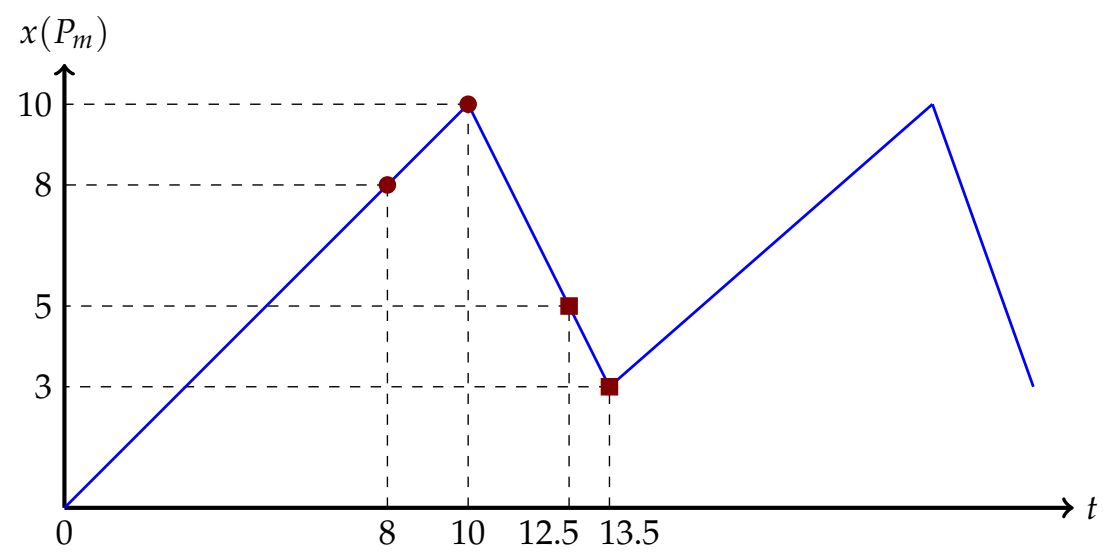

Figure 3.6.: Evolution of content of continuous place $P_{m}$ over time, assuming that none of the general transitions has fired.

\subsubsection{Overflow place: dynamic transitions}

In some application areas we may need to allow a certain continuous place to overflow, which is not allowed in the current setting, as each continuous place is associated with an upper boundary. Overflows are useful when modelling sedimentation tanks, in water treatment facilities, which is one of the application areas toward which this dissertation is aimed (Chapter 8). In this section we will use dynamic transitions and guard arcs to establish a new component, the so-called overflow place.

In case of a completely filled continuous place, the overflow is defined as the difference of the actual rate of the inflow and the outflow of that place. This can be done using dynamic transitions. More specifically, an overflow place is a structure that when the continuous place reaches its upper boundary, a dynamic transition becomes enabled with a rate that equals the difference of the actual rate of all the incoming and outgoing transitions.

Figure 3.7 shows the model of an overflow place. Transitions $T_{i}$ and $T_{0}$ represent the overall input and output to the continuous place $P_{0}$, respectively. Therefore, we denote the overall actual inflow rate by $r\left(T_{i}\right)=\sum f_{i} \leq \phi_{S t}^{\mathcal{T}}\left(T_{i}\right)$ and the overall actual outflow rate by $r\left(T_{o}\right)=$ $\sum f_{o} \leq \phi_{S t}^{\mathcal{T}}\left(T_{o}\right)$. Whenever the continuous place, $P_{o}$ reaches a certain boundary $B$, the immediate transition, $T_{f}$, connected to it via the guard arc will fire, and as a result the dynamic transition, $T_{d}$ becomes enabled. Note that the rate of this transition is adapted according to the inflow and outflow of the main continuous place, i.e., $\phi_{D y}^{\mathcal{T}}\left(T_{d}\right)=$ $h\left(r\left(T_{i}\right), r\left(T_{o}\right)\right)=r\left(T_{i}\right)-r\left(T_{o}\right)=\sum f_{i}-\sum f_{o}$, in which $\sum f_{i}$ and $\sum f_{o}$ are the total input and output to $P_{0}$, respectively. Also, whenever the fluid 


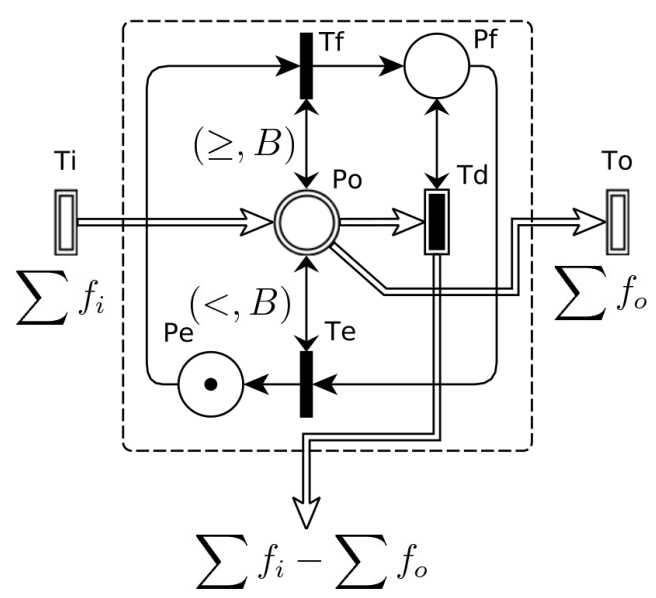

Figure 3.7.: Modelling of an overflow place using HPnG primitives.

level in the continuous place, $P_{o}$ would reach blow the boundary $B$, due to a change either in inflow or outflow, the connected immediate transition, $T_{e}$, will fire and the dynamic transition is disabled, and hence no overflow.

For ease of modelling, we represent an overflow place graphically as follows, in Figure 3.8, in which the sum of inflow and outflow rates are denoted by $\sum f_{i}$ and $\sum f_{0}$, respectively. Moreover, the overflow rate is shown by $R_{o}=\sum f_{i}-\sum f_{o}$, and distinguished from ordinary outgoing transitions by a shaded rectangle connected to the overflow place.

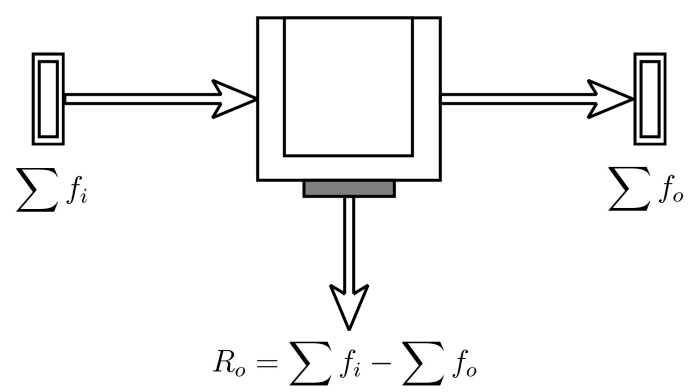

Figure 3.8.: Overflow place component.

\subsection{CONFLICT RESOLUTION FOR DISCRETE TRANSITIONS}

The concept of conflicts for both discrete and continuous timed Petri nets were discussed in Sections 2.1.4 and 2.3.2. Since now we are allow- 
ing both discrete and continuous places/transitions in HPnGs, we need to consider conflicts for both cases. In this section we discuss conflicts among discrete transitions, and the policies we use to resolve these conflicts. In Section 3.5, we explore the conflict resolution algorithm for continuous transitions, the so-called rate adaptation algorithm.

A conflict among two discrete transitions can occur when a set of transitions can fire at the same time, but depending on which order they fire the marking of the Petri net is going to be different. This can happen for instance when two transitions are sharing a resource, i.e., a token in a discrete place. This is depicted in Figure 3.9, which is repeated here from Chapter 2. Therefore, we need to specify a policy for resolving conflicts. For the case of conflicts among discrete transitions we may have two different policies for determining the order by which transitions fire.

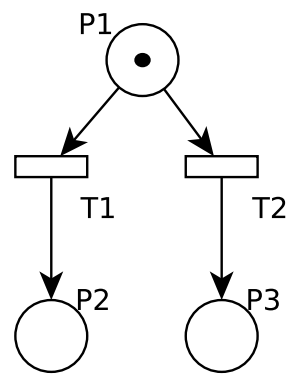

Figure 3.9.: A conflict between transitions $T_{1}$ and $T_{2}$.

In the following we assume $\mathcal{T}_{E}^{D}$ is the set of enabled discrete transitions which can fire at the same time.

PRIORITIZING. For this policy we assume the function $\phi_{p}^{\mathcal{T}}$ assigns a unique priority to all discrete transitions. Then in case of conflicts we always choose the discrete transition with the highest priority. More formally we choose $T^{d} \in \mathcal{T}_{E}^{D}$ if and only if $\phi_{p}^{\mathcal{T}}\left(T^{d}\right)>\phi_{p}^{\mathcal{T}}\left(T_{i}^{d}\right)$ for all $T_{i}^{d} \in \mathcal{T}_{E}^{D} \backslash\left\{T^{d}\right\}$.

PRoвAbilistic Selection. For this we treat the function $\phi_{p}^{\mathcal{T}}$ as a weight function, hence, we do not need to assign unique priorities to all discrete transitions. The idea is that when a set of transitions is in conflict, those with higher weights have higher probability to be chosen. More formally, each transition $T^{d} \in \mathcal{T}_{E}^{D}$ is chosen to fire with probability:

$$
p_{T^{d}}=\frac{\phi_{p}^{\mathcal{T}}\left(T^{d}\right)}{\sum_{T_{i}^{d} \in \mathcal{T}_{E}^{D}} \phi_{p}^{\mathcal{T}}\left(T_{i}^{d}\right)} .
$$


Different policies allow for different analysis approaches. We will employ each of the above policies in later chapters, based on the approach we undertake toward analysis of HPnGs. Particularly, the approach presented in Chapter 5 uses prioritizing, and in Chapter 7 we use probabilistic selection.

\subsection{CONFLICT RESOLUTION FOR CONTINUOUS TRANSITIONS}

The main goal in this section is to adapt rates of continuous transitions to avoid both underflow and overflow. For this we first formalize the concept of conflict among sets of continuous transitions with respect to a continuous place. As mentioned earlier in Definition 2.6, a set of transitions are in conflict if the rate of at least one of them has to be slowed down, due to the rate of the others. This has to be done when with the current actual rates of connected continuous transitions there is either an overflow or an underflow in a continuous place. ${ }^{4}$ In the following we formalize these two concepts:

Definition 3.7 (Overflow and underflow). There is an overflow in a continuous place $P^{c}$ if the following two conditions hold:

1. $x\left(P^{c}\right)=\phi_{b}^{\mathcal{T}}\left(P^{c}\right)$,

2. $d\left(P^{c}\right)>0$,

where $d\left(P^{c}\right)$ is the drift of the place introduced in Equation (3.2), and $\phi_{b}^{\mathcal{T}}\left(P^{c}\right)$ is the upper boundary assigned to $P^{c}$, introduced in Section 3.1. We have an underflow in a continuous place $P^{c}$ if the following two conditions hold:

1. $x\left(P^{c}\right)=0$,

2. $d\left(P^{c}\right)<0$.

Note that the drift of a place should be strictly positive or negative to satisfy the condition for overflow or underflow. Indeed our goal is to adapt the rates of incoming or outgoing transitions in such a way that the drift of the place becomes zero, hence, both overflow and underflow are avoided.

In case of an overflow, the sum of actual rates of transitions in $\mathcal{I}_{P}\left(P^{c}\right)$ should be reduced to match the sum of actual rates of transitions in $\mathcal{O}_{P}\left(P^{c}\right)$. The same holds for the underflow case, where the sum of rates of transitions in $\mathcal{O}_{P}\left(P^{c}\right)$ should be reduced to match the sum of rates of transitions in $\mathcal{I}_{P}\left(P^{c}\right)$. We call this matching process rate adaptation.

Now we define the concepts of structural and actual conflicts.

4 Recall that in our formal definition of HPnGs, a continuous place is provided with upper and lower boundaries, and its content is not allowed to exceed any of these boundaries This should not be confused with the overflow place structure introduced in Section 3.3.3. 
Definition 3.8 (Structural conflict). There is a structural conflict in continuous place $P^{c}$, if one of the following two conditions holds:

- $\left|\mathcal{I}_{P}\left(P^{c}\right)\right|>1$.

- $\left|\mathcal{O}_{P}\left(P^{c}\right)\right|>1$.

Note that if the place involved in a structural conflict is neither in overflow, nor in underflow, there is no need for rate adaptation. A structural conflict is just an indicator, that there may be a need for conflict resolution later for this structure. Rate adaptation is needed in case of an actual conflict:

Definition 3.9 (Actual conflict). There is an actual conflict in continuous place $P^{c}$, if one of the following two conditions holds:

- there is an overflow in $P^{c}$ and $\left|\mathcal{I}_{P}\left(P^{c}\right)\right|>1$,

- there is an underflow in $P^{c}$ and $\left|\mathcal{O}_{P}\left(P^{c}\right)\right|>1$.

In this case transitions in $\mathcal{I}_{P}\left(P^{c}\right)$ or $\mathcal{O}_{P}\left(P^{c}\right)$ are in conflict. We also refer to the conflict structure as $\left(P^{c}, \mathcal{I}_{P}\left(P^{c}\right)\right)$ in case of the first condition and $\left(P^{c}, \mathcal{O}_{P}\left(P^{c}\right)\right)$ in case of the second.

Let the continuous place $P^{c}$ satisfies the underflow conditions, for instance. If there is only one output transition connected to it, i.e., $\left|\mathcal{O}_{P}\left(P^{c}\right)\right|=1$, then the rate of this transition, say $T^{c}$, should be reduced to match the overall input rate, i.e., $r\left(T^{c}\right)=f_{\text {in }}\left(P^{c}\right)$. On the other hand, if the number of output transitions is larger than one, i.e., $\left|\mathcal{O}_{P}\left(P^{c}\right)\right|>1$, the available flow, $f_{\text {in }}\left(P^{c}\right)$, needs to be distributed among the transitions in $\mathcal{O}_{P}\left(P^{c}\right)$. An instance of an actual conflict in case of underflow is shown in Figure 3.10, which is repeated here from Section 2.3.2.

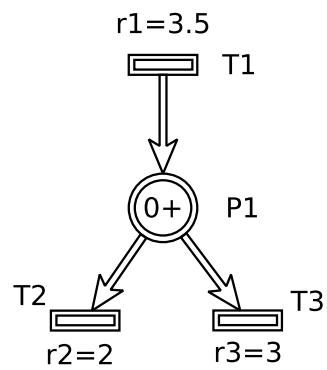

Figure 3.10.: Demonstration of conflict among continuous transitions $T_{2}$ and $T_{3}$.

There are two main approaches for deciding how the available flow should be distributed among conflicting transitions: resolution using 
only priorities, and resolution using priorities and sharing. This is done using the assigned priorities and shares of the arcs connecting $P^{c}$ to transitions in $\mathcal{O}_{P}\left(P^{c}\right)$ (in case of underflow) or $\mathcal{I}_{P}\left(P^{c}\right)$ (in case of overflow), which are specified by the mappings $\phi_{p}^{\mathcal{A}}$ and $\phi_{s}^{\mathcal{A}}$, in Section 3.1.

\subsubsection{Conflict resolution using only priority}

Resolution using only priority takes the strong assumption that whenever there is an actual conflict among a set of transitions, each of them is associated with a unique priority. More formally, in case of underflow, it assumes that if there is a conflict structure $C_{P^{c}}=\left(P^{c}, \mathcal{O}_{P}\left(P^{c}\right)\right)$, the function $\phi_{p}^{\mathcal{A}}$ assigns a unique priority to each arc $\left\langle P^{c}, T\right\rangle$ for each transition $T \in \mathcal{O}_{P}\left(P^{c}\right)$. Therefore, in a conflict structure $C_{P^{c}}$, the number $\phi_{p}^{\mathcal{A}}\left(\left\langle P^{c}, T\right\rangle\right)$ is the priority of transition $T$. Now the available flow is distributed among transitions such that transitions with higher priorities are served first, i.e., transitions with lower priority do not receive any flow unless those with higher priorities can work on their nominal rate.

This procedure is given in Algorithm 3.1 for only the case of underflow. In lines 1-5, an iteration takes place over all continuous transitions. For a continuous transition if all its connected input places have positive content (line 2), it can fire with its maximum speed, therefore in line 3 the actual rate of such transition is set to its nominal rate. On the other hand if any of the connected input places is empty, the actual rate of the transition may be needed to be adapted according to the inflow of connected continuous places. So for the moment actual rate of such transition is set to zero.

The remaining process is as follows. We iterate over all empty continuous places (line 7), since these are susceptible places for underflow, and consequential actual conflict. Through lines 8-15, the available flow, i.e., the inflow to each of such continuous places, is distributed among its output continuous transitions, in order of their priorities. More precisely, while the there is available flow (line 10), at each iteration the connected output transition with highest priority is selected in line 11, and its actual rate is set to the maximum possible value, i.e., the maximum among available flow, and its nominal rate (line 12). After this in line 13 the available flow is updated, since part of it is assigned to the highest priority continuous transition. Finally, the transition that its actual rate is set, is removed from the set of output transitions in line 14 . After it is ensured that the entire available flow is distributed (i.e., the loop condition in line 10 is no longer satisfied), the actual rate of the remaining output transitions, i.e., those with lower priorities, is set to zero, in line 15. This process is continued until the actual rate of no continuous transition is changed after an iteration over all empty continuous 
places, in other words, until when there is no under flow in the network, and all transitions can fire according to their maximum possible rate.

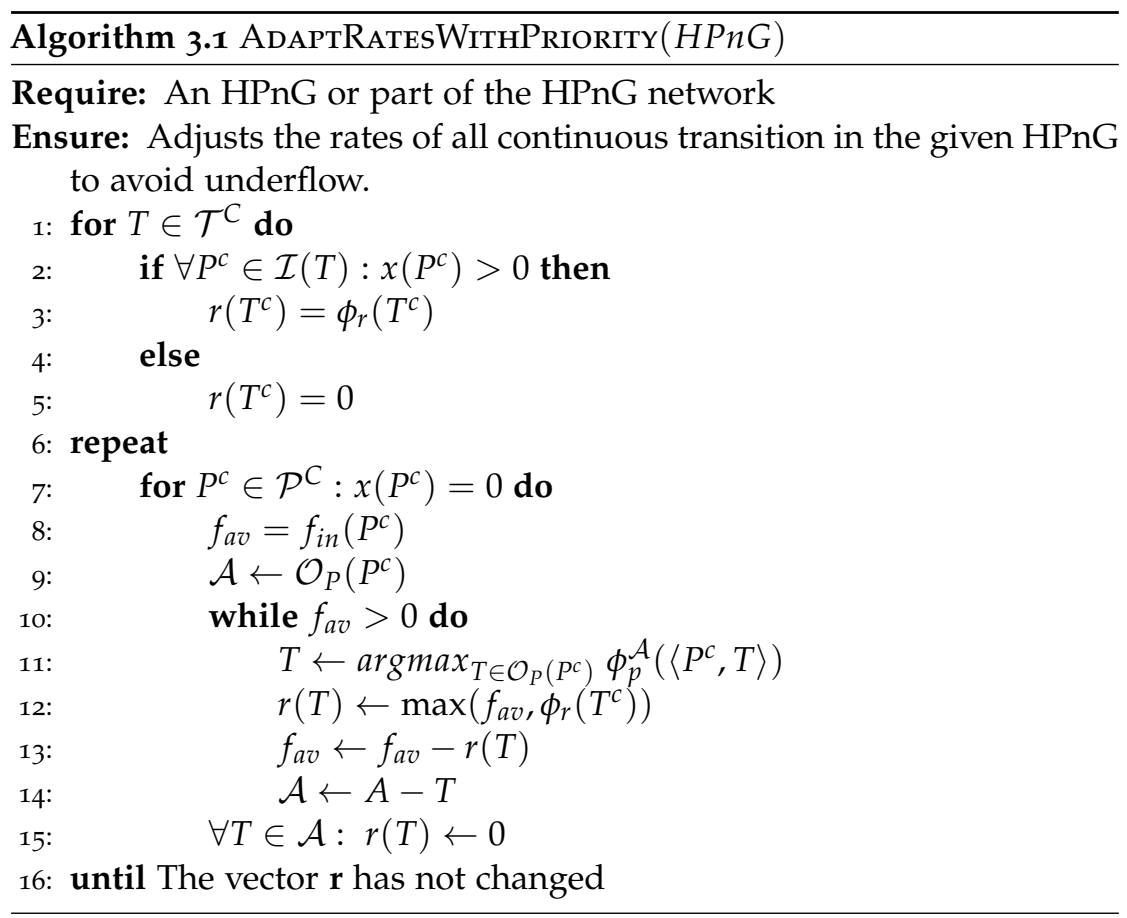

The above algorithm ensures that all transitions are going to fire at their maximum possible rates. Through iteration in lines 6-16, if certain conditions hold, as we will discuss later (cf. Hypothesis 3.1 in Subsection 3.5.2.2), the actual rates of transitions are always increasing, and since there is upper bound for the actual rates of transitions, i.e., their nominal rates, the algorithm will always terminate. 5 For a counter example see Example 1 in Subsection 3.5.2.2.

In spite of simplicity, the above algorithm can also be sometimes inefficient. Figure 3.11 gives an example for such a case. In this example, $T_{1}$ and $T_{2}$ are in conflict as $P_{1}$ is underflowing. However, $T_{3}$ can work at its full rate, i.e., $2+\epsilon$, because $P_{2}$ is not empty, however, since other places are empty the actual rates of the rest of the transitions are set to zero at the start of the algorithm. Now the available flow to $P_{1}$ is $2+\epsilon$. Assume $T_{1}$ has priority over $T_{2}$, therefore the algorithm sets $r\left(T_{1}\right)=2$ and $r\left(T_{2}\right)=\epsilon$. Now the available flow to $P_{3}$ is $\epsilon$, therefore, we will have

5 Note that the amount of increase of actual rate of each transition is determined by the actual rates of other transitions. Hence it can be guaranteed that this increase amount is bounded away from a constant, and therefore the convergence is guaranteed. In a more technical terms, an increasing sequence sequence $a_{n}$, which is bounded from above, will always converge, if there exist $\epsilon>0$ s.t. $a_{i}-a_{j}>\epsilon$ for all $i>j$. This is the case for this algorithm. 
$r\left(T_{4}\right)=\epsilon$. But this causes an increase in available flow of $P_{1}$, which will be given to $T_{2}$, therefore $r\left(T_{2}\right)=2 \epsilon$. This again in turn increases the available flow of $P_{3}$, hence again an $\epsilon$ increase in actual rate of $T_{4}$. This will continue until one of the transitions reaches its nominal rate. In this example the total number of iterations would be $1 / \epsilon$, which can be large.

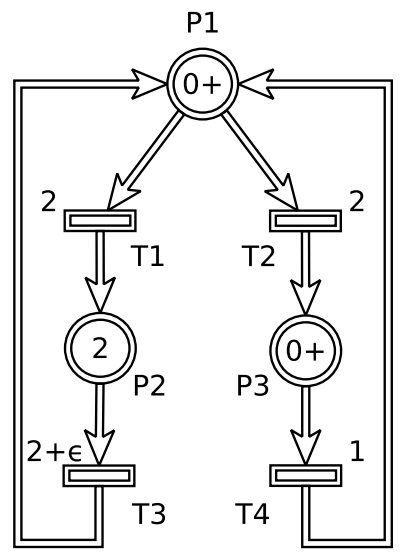

Figure 3.11.: Example of conflict resolution using only priority.

To avoid examples like the above, and also to remove the extra mentioned conditions for termination, instead of the proposed iterative method in Algorithm 3.1, one can use linear programming. In this approach the sum of actual rates of transitions are maximized, with respect to a constraint set. This constraint set includes, for instance, the condition that actual rates of transitions should not exceed their nominal rates, and that places in underflow and overflow should have non-negative and non-positive drifts (Equation (3.2)), respectively. The latter constrains means both underflow and overflow are avoided. We do not explore resolution using linear programming in this dissertation, and refer to [34], for a complete treatment. However, we stress on the fact that the only assumption this approach needs to establish, is the unique priority among transitions involved in an actual conflict, and no presupposition or constrain on the structure of the network is needed. From now on we assume that this algorithm is employed for conflict resolution using only priorities, therefore no extra condition is imposed on the network. 


\subsubsection{Conflict resolution using priority and sharing}

We first consider and provide an algorithm for conflict resolution among a set of transitions connected to a single continuous place. After this we discuss how this conflict resolution will influence, and propagate through the entire network, and how the structure of the network is important in being able to perform the resolution. For simplicity, in the following we consider only the case of underflow, as overflow can also be handled similarly.

\subsubsection{Conflict in a single place}

We start the discussion of the rate adaptation algorithm, by first investigating the conflict resolution for only one place. The transitions connected via highest priority arcs are considered first, and if there is enough flow available (from the input transitions), all transitions with the highest priority can still work at their maximum rates. Otherwise, their actual rates are reduced according to the share of the connecting arcs. This idea is formalized in what follows.

In order to treat the nominal rates of static and dynamic continuous transitions in the same way, the function $\phi_{r}: \mathcal{T}^{C} \rightarrow \mathbb{R}^{+}$is defined as follows:

$$
\phi_{r}\left(T^{\mathcal{c}}\right)= \begin{cases}\phi_{S t}^{\mathcal{T}}\left(T^{c}\right) & T^{\mathcal{c}} \in \mathcal{T}^{S t}, \\ \phi_{D y}^{\mathcal{T}}\left(T^{\mathcal{C}}\right)\left(\mathbf{r}_{S t}\right) & T^{\mathcal{c}} \in \mathcal{T}^{D y},\end{cases}
$$

where $\mathbf{r}_{S t} \in \mathbb{R}^{\left|\mathcal{T}^{S t}\right|}$ is the current vector of actual rates of static continuous transitions. Therefore, function $\phi_{r}$ returns the nominal rate, i.e., the maximum working speed of the given transition, depending on its type. We also define the set function $\phi_{r}: 2^{\mathcal{T}^{C}} \rightarrow \mathbb{R}^{+}$over a set of continuous transitions which returns the sum of their nominal rates:

$$
\phi_{r}(Q)=\sum_{T^{c} \in Q} \phi_{r}\left(T^{c}\right) .
$$

We define the set function $\phi_{s}^{P^{c}}: 2^{\mathcal{T}^{C}} \rightarrow \mathbb{R}^{+}$, which returns the sum of the shares of the arcs connecting the continuous place $P^{c}$ to the continuous transitions:

$$
\phi_{s}^{P^{c}}(Q)=\sum_{T^{c} \in Q} \phi_{s}^{\mathcal{A}}\left(\left\langle P^{c}, T^{c}\right\rangle\right) .
$$

Let $Q_{l}\left(P^{c}\right) \subseteq \mathcal{O}_{P}\left(P^{c}\right)$ be the set of continuous output transitions connected to $P^{c}$, via arcs of priority level $l$, i.e., $Q_{l}\left(P^{c}\right)=\left\{T^{c} \in \mathcal{O}_{P}\left(P^{c}\right)\right.$ : $\left.\phi_{p}^{\mathcal{A}}\left(\left\langle P^{c}, T^{c}\right\rangle\right)=l\right\}$, and let $l_{\text {max }}=\max _{T^{c} \in \mathcal{O}_{P}\left(P^{c}\right)} \phi_{p}^{\mathcal{A}}\left(\left\langle P^{c}, T^{c}\right\rangle\right)$, be the maximum priority level of arcs connecting $P^{c}$ to output transitions. Algorithm 3.2, which is based on [26], demonstrates how the available flow $f_{\text {in }}\left(P^{c}\right)$, can be distributed among connecting output transitions. 


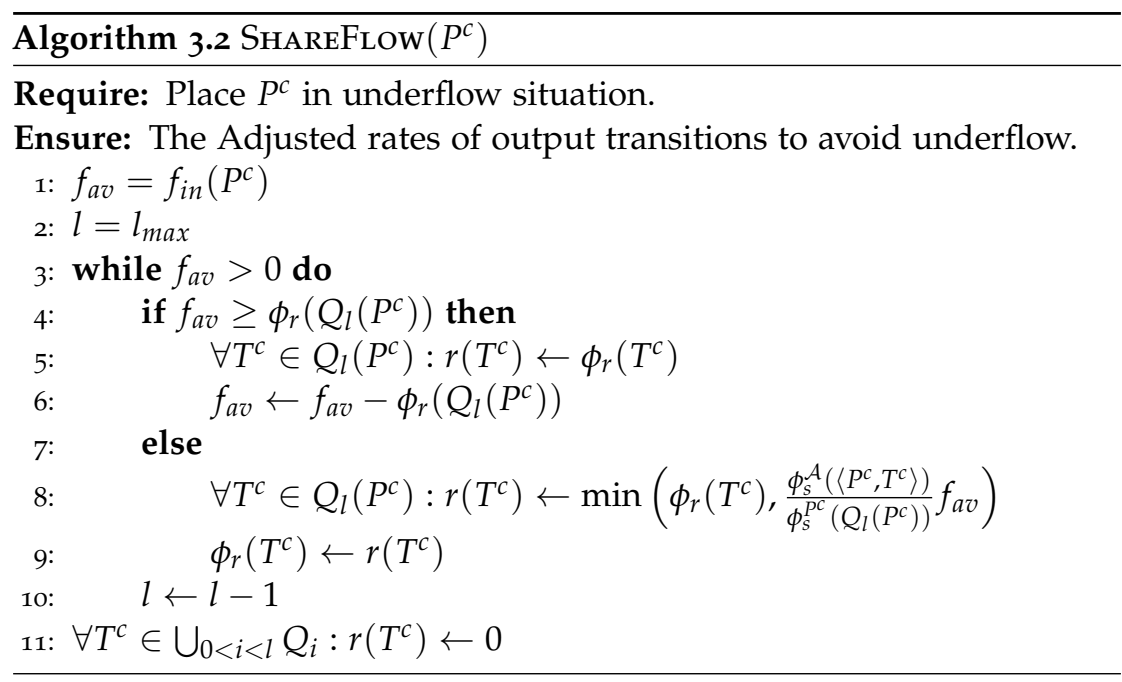

Algorithm 3.2, assigns the available flow $f_{a v}$ (which initially equals $\left.f_{\text {in }}\left(P^{c}\right)\right)$ to the transitions, starting with the highest priorities. The process goes on until no available flow remains (line 3 ). If $f_{a v}$ is enough such that all transitions in priority level $l$ can fire at their maximum speed (condition in line 4 ), then we assign all the transitions in $Q_{l}\left(P^{c}\right)$ with their nominal rates (line 5). After this we reduce the available flow by the already assigned flow (line 6). If we reach a priority level such that the remaining available flow is not enough so that all transitions can fire at their nominal speed, i.e., the condition in line 4 is not satisfied, then the available flow is distributed proportional to the share of the connecting arcs (line 8). Note the minimum operator in line 8, since we need to make sure $r\left(T^{c}\right) \leq \phi_{r}\left(T^{c}\right)$. Finally, when we are sure that no input flow remains $\left(f_{a v}=0\right)$, we set the rate of all the transitions belonging to lower priority levels to zero (line 10).

We illustrate the above algorithm for the conflict case in Figure 3.12. Nominal rates of transitions are shown besides each of them. As can be seen place $P_{1}$ satisfies the conditions for underflow: it is empty and its input flow (9) is less than the demand which is the sum of all connected output transition rates(15). There are four priority levels as illustrated in the figure by dashed boxes. All the characteristics related to transitions, including nominal rate, priorities, and shares are given in Table 3.1.

The steps which are performed by Algorithm 3.2, for this example, are given in Table 3.2. Algorithm 3.2, starts with $f_{a v}=9$. Transitions $T_{2}$ and $T_{3}$, have the highest priority level, which is 4 . Since $f_{a v} \geq \phi_{r}\left(Q_{4}\left(P_{1}\right)\right)$ (condition in line 4) both transitions can work at their full speed. By subtracting the assigned flow from the available flow, i.e., the new $f_{a v}=6$, the algorithm proceeds with priority level 3, which includes transitions $T_{4}$ and $T_{5}$. For this priority level the condition in line 4 is no longer 


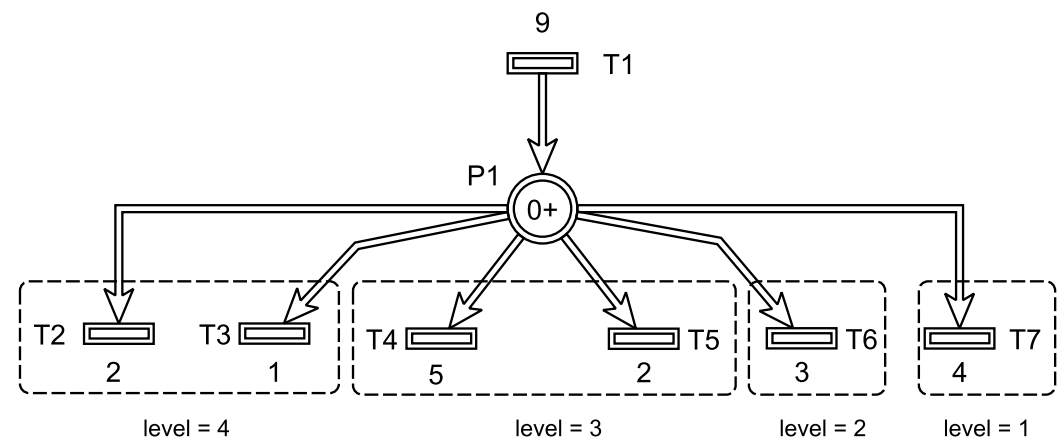

Figure 3.12.: Example of conflict resolution.

\begin{tabular}{l|cc|cc|c|c} 
& $T_{2}$ & $T_{3}$ & $T_{4}$ & $T_{5}$ & $T_{6}$ & $T_{7}$ \\
\hline \hline$\phi_{p}^{\mathcal{A}}\left(\left\langle P^{c},.\right\rangle\right)$ & \multicolumn{2}{|c|}{4} & \multicolumn{2}{|c|}{3} & 2 & 1 \\
\cline { 2 - 7 }$\phi_{s}^{\mathcal{A}}\left(\left\langle P^{c},.\right\rangle\right)$ & $\mathbf{1}$ & $\mathbf{1}$ & $\mathbf{1}$ & $\mathbf{2}$ & $\mathbf{1}$ & $\mathbf{1}$ \\
\cline { 2 - 7 }$\phi_{r}()$. & $\mathbf{2}$ & $\mathbf{1}$ & 5 & 2 & 3 & 1 \\
\hline Computed $r()$. & 2 & $\mathbf{1}$ & $\mathbf{2}$ & $\mathbf{2}$ & $\mathbf{2}$ & $\mathrm{O}$
\end{tabular}

Table 3.1.: Characteristics of the transitions in Figure 3.12 in rows 2-4, and their computed actual rates with Algorithm 3.2 given in row 5 .

satisfied. Using shares of transitions, their actual rates are computed according to the formula in line 8 of the algorithm. This is also given in the third row of Table 3.2. Now for the priority level 2, we have $f_{a v}=2$, which goes to transition $T_{6}$, and priority level 1 , receives no flow.

\begin{tabular}{c|c|c|c} 
& $f_{a v}$ & $Q_{l}\left(P_{1}\right)$ & Assignments \\
\hline \hline$l=4$ & 9 & $\left\{T_{2}, T_{3}\right\}$ & $r\left(T_{2}\right)=2, r\left(T_{3}\right)=1$ \\
\hline$l=3$ & 6 & $\left\{T_{4}, T_{5}\right\}$ & $\begin{array}{c}r\left(T_{4}\right)=\min \left(\frac{1}{3} \times 6,5\right)=2, \\
r\left(T_{5}\right)=\min \left(\frac{2}{3} \times 6,2\right)=2\end{array}$ \\
\hline$l=2$ & 2 & $\left\{T_{6}\right\}$ & $r\left(T_{6}\right)=2$ \\
\hline$l=1$ & 0 & $\left\{T_{7}\right\}$ & $r\left(T_{7}\right)=0$
\end{tabular}

Table 3.2.: Demonstration of steps of Algorithm 3.2, for the example of Figure 3.12.

\subsubsection{The entire network}

Being able to resolve the conflict for one place, one may think of iterating over all continuous places and resolve the conflicts one by one. Unfortu- 
nately, this is not always possible, because once we are adjusting the rate for a transition it may cause an overflow or underflow, hence, a conflict in another place, and resolving the conflict in the new place may cause a new conflict in the previously resolved place. We explain this in the following two examples.

Example 1. This example demonstrates the problem of having one transition being present in two conflict. Figure 3.13, shows two places $P_{1}$ and $P_{2}$ in state of underflow, therefore there are two conflict structures, present at the same time. Moreover, in the conflict structure regarding $P_{1}$, let the priorities being assigned to the output transitions through arcs, be such that, $T_{1}$ has priority over $T_{2}$. With slight abuse of notation, we denote this by $T_{1}>T_{2}$. In the conflict regarding $P_{2}$, we have $T_{2}>T_{3}$. Now assume that the conflict resolution as demonstrated in Algorithm 3.2 is performed first on $P_{1}$. The resulting rate for both $T_{1}$ and $T_{2}$, is 1 . Now performing the algorithm on $P_{2}$ to resolve the conflict, will compute the rates for $T_{2}$ and $T_{3}$, as 2 and 1 , respectively. This will cause underflow again in $P_{1}$. Therefore, as the example shows, the iterative application of Algorithm 3.2 may not terminate.

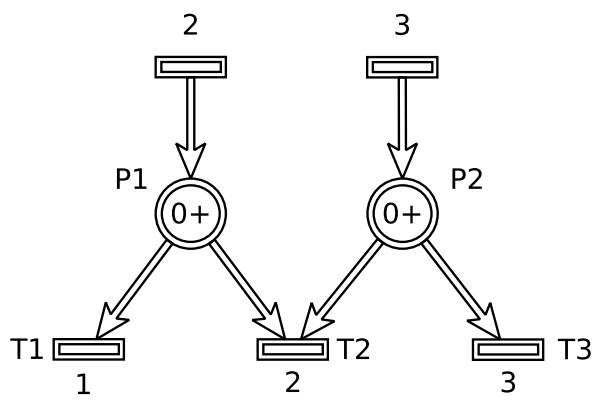

Figure 3.13.: Example of conflict resolution.

Example 2. This example shows how a feedback to a place in a conflict structure, may cause the iterative application of Algorithm 3.2 not to terminate. In Figure 3.14 places $P_{1}$ and $P_{3}$, are in state of underflow. Assume transitions $T_{1}$ and $T_{2}$ are connected via arcs assigning equal sharing to both of them, hence, both receive half of the available flow from $P_{1}$. In the begining, the only transition which can fire at full speed is $T_{3}$, since its input place is not empty. Therefore, the inflow to $P_{1}$ is 1 , which is shared equally among $T_{1}$ and $T_{2}$, i.e., $r\left(T_{1}\right)=r\left(T_{2}\right)=1 / 2$. Now the inflow of $P_{3}$ is positive $\left(f_{\text {in }}=1 / 2\right)$, which means $T_{4}$ can fire, hence, $r\left(T_{4}\right)=1 / 2$. This will add the amount $1 / 2$ to the inflow to $P_{1}$, which again needs to be distributed equally among $T_{1}$ and $T_{2}$, therefore, $r\left(T_{1}\right)=r\left(T_{2}\right)=1 / 2+1 / 4$. This in turns adds the value of $1 / 4$ to the inflow of $P_{3}$, which needs to be added to the rate of $T_{4}: r\left(T_{4}\right)=1 / 2+1 / 4$. 
So again, $P_{1}$ will have available inflow, and this process continues, and will never converge to a stable value. However, the stable solution for this example is: $r\left(T_{1}\right)=r\left(T_{2}\right)=r\left(T_{3}\right)=r\left(T_{4}\right)=1$, as the algorithm is also trying to converge to. ${ }^{6}$ This example is inspired by one of the eamples in [34].

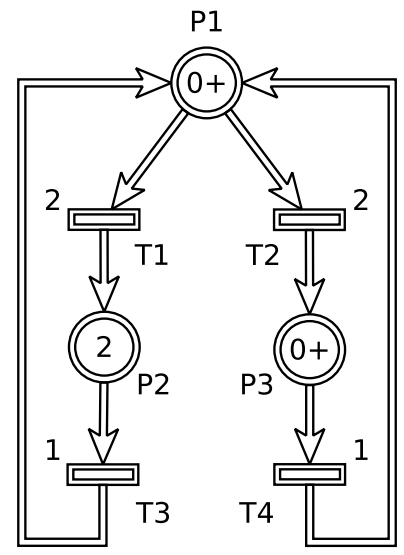

Figure 3.14.: Example of conflict resolution.

The above two examples show that we need to make specific assumptions about the structure of the network before devising the iterative algorithm. We state and formalize two hypotheses in the following.

The first hypothesis states that no transition is involved in more than one conflict:

Hypothesis 3.1 ( [34]). For any conflict structure $C_{P^{c}}=\left(P^{c}, \mathcal{O}_{P}\left(P^{c}\right)\right)$, let $\mathcal{I}_{P}\left(C_{P^{c}}\right)=\cup_{T \in \mathcal{O}_{P}\left(P^{c}\right)} \mathcal{I}_{T}(T)$, be the set of all input places to transitions involved in conflict. Then, the only place in $\mathcal{I}_{P}\left(C_{P^{c}}\right)$ which is in conflict is $P^{c}$.

The next hypothesis states a condition on the feedbacks to conflicting structures. Informally the hypothesis states that none of the components of a conflicting structure should involve in a feedback. This means the data for resolving a conflict does not depend on the result of the conflict resolution. Therefore, with respect to a conflict structure $C_{P^{c}}=\left(P^{c}, \mathcal{O}_{P}\left(P^{c}\right)\right)$, the entire network can be divided into three disjoint sub-networks:

(1) the components "above" the conflict structure denoted $\mathcal{U}\left(C_{P^{c}}\right)$,

(2) the components "below" the conflict structure denoted $\mathcal{D}\left(C_{P^{c}}\right)$, and

6 Note that the actual rates of transitions $T_{1}, T_{2}$, and $T_{4}$, form the geometric series, $\frac{1}{2}+$ $\frac{1}{4}+\frac{1}{8}+\cdots$. Although these series converge, however, the algorithm will never terminate assuming unlimited precision. 
(3) the components the conflict structure $C_{P^{c}}$ itself.

These are illustrated in Figure 3.15.

Hypothesis 3.2 ( [34]). The given HPnG can be be divided into three subnetworks, such that:

1. There is no arc from components of $C_{P^{c}}$ to the components of $\mathcal{U}\left(C_{P^{c}}\right)$, there is no arc from $P^{c}$ to $\mathcal{D}\left(C_{P^{c}}\right)$, and there is no arc from conflicting transitions in $\mathcal{O}_{P}\left(P^{c}\right)$ to $P^{c}$.

2. There is no arc from elements in $\mathcal{D}\left(C_{P^{c}}\right)$ to the elements of $\mathcal{U}\left(C_{P^{c}}\right)$, and $C_{P^{c}}$.

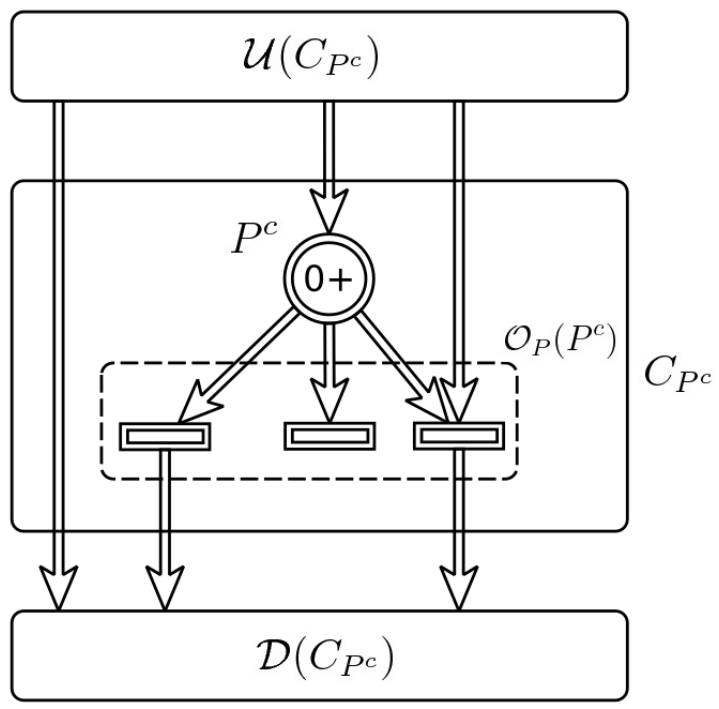

Figure 3.15.: Demonstration of Hypothesis 3.2.

Checking whether the above hypotheses are satisfied, is based on polynomially decidable graph traversal algorithms, which are beyond the scope of this dissertation. However, although we allude to the verification process in what follows, we refer to Appendix L of [34], for complete treatment of the algorithm.

Having formalized the above two hypotheses, we are ready to devise the algorithm for the rate adaptation in the entire network. We first investigate the algorithm for a network in which there is only one actual conflict, for which sharing is used for resolution. We then generalize the algorithm for a network with several sharing in different actual conflicts. The algorithms presented in the following sections can be seen as summary of the speed calculation algorithms presented in [34]. 


\section{Conflict resolution for only one sharing}

In this section we assume there is only one conflict structure, say $C_{P^{c}}=$ $\left(P^{c}, \mathcal{O}_{P}\left(P^{c}\right)\right)$, in the entire network, for which resolution is done using sharing among transitions. Moreover, we assume both Hypotheses 3.1 and 3.2 hold. In this case the network has the structure given in Figure 3.15. This means that all the data needed to adapt the rates of transitions in $\mathcal{O}_{P}\left(P^{c}\right)$ are available, if one knows the status of the sub-network $\mathcal{U}\left(C_{P^{c}}\right)$. Since it is assumed that $C_{P^{c}}$ is the only conflict for which sharing is used, to compute the rates of transition in $\mathcal{U}\left(C_{P^{c}}\right)$, one can use the resolution only using priority ( AdAPTRATESWITHPRIORITY), as presented in Section 3.5.1. After this, we can safely apply Algorithm 3.2 on $P^{c}$, to compute the rates of transitions in $\mathcal{O}_{P}\left(P^{c}\right)$. Now all the data for computing rates of transitions in $\mathcal{D}\left(C_{P^{c}}\right)$ are available, hence, we can use AdaptRatesWithPriority again. This idea is given in Algorithm 3.3.

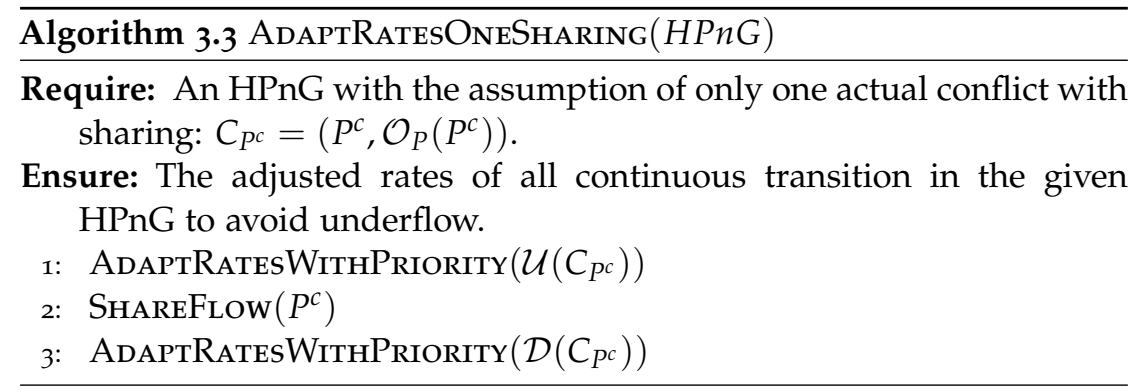

The complete rate adaptation algorithm

Now we consider the general case, i.e., when there are several conflict structures among which sharing is used for conflict resolution. In this section we also assume that both Hypotheses 3.1 and 3.2 hold. The idea is to adapt the rates of all transitions "above" each conflict structure, before adapting the rates of transitions in conflict. This is indeed possible because of Hypothesis 3.2. Moreover, Hypothesis 3.1 assumes that after adapting rates of transitions involved in a sharing, their rates are not going to change due to the resolution of other conflicts.

In order to do this ordered rate adaptation, we need to come up with an ordering of components of the given network. For this we use the graph of relations among conflicts [34], which is built based on the given original network. Each vertex in this graph represents a maximal set of transitions in structural conflict (if a transition is not in a conflict it is represented singularly by a vertex). There is an edge between two vertices $v_{1}$ and $v_{2}$ of this graph if there is path of length at least two (with an intermediate place) in the original network, between a transition in $v_{1}$ and a transition in $v_{2}$. This is formalized as follows. 


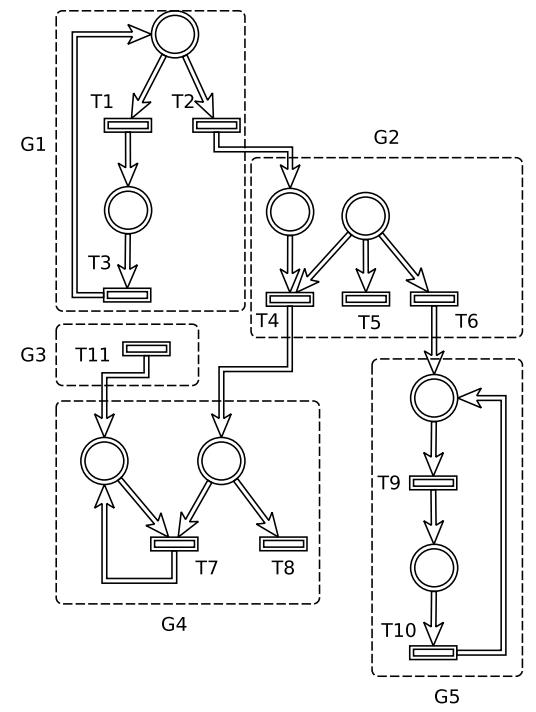

(a) An example HPnG.

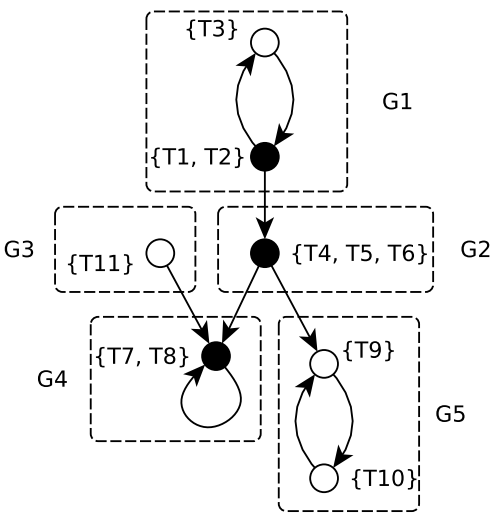

(b) The graph of conflict relations.

Figure 3.16.: An example of building the graph of relations among conflicts for a given network.

Definition 3.10. The graph of relations among conflicts, is the directed graph $G=(V, E)$ such that:

- Each $v_{i} \in V$ is the maximal set of transitions in a structural conflict, or is a singular transition not involved in a conflict.

- $\left(v_{1}, v_{2}\right) \in E$ iff there exist a continuous place P such that there is a path $T_{1} \rightarrow P \rightarrow T_{2}$ in the original network, for $T_{1} \in v_{1}$ and $T_{2} \in v_{2}$.

Note that because of Hypothesis 3.1, each transition belongs only to one vertex of $G$, therefore, $V$ forms a partition of $\mathcal{T}^{C}$.

Figure 3.16a shows an example HPnG. Only the continuous part is shown here, since the discrete part is irrelevant to the content of this section. Figure 3.16b, depicts the corresponding graph of relations among conflicts. As can be seen, two types of vertices are distinguished: black vertices are those transitions involved in a structural conflict, and white vertices are singular transitions which are not in any conflict.

Now consider the strongly connected components 7 (SCC) of G. One can easily prove the following proposition as the necessary and sufficient condition for Hypothesis 3.2 to hold.

7 A strongly connected components in a directed graph is a maximal subset of its vertices such that there is a path from each of the vertices to the others. 
Proposition 3.1. Hypothesis 3.2 is satisfied, if and only if, for each actual conflict $C_{P^{c}}=\left(P^{c}, \mathcal{O}_{P}\left(P^{c}\right)\right)$, the vertex associated with $\mathcal{O}_{P}\left(P^{c}\right)$ in the graph of relations among conflicts, is the only element of an SCC, and it does not contain a self-loop.

This property is easy to see, however, we refer to [34] (Appendix L) for the proof. One can use the above proposition to verify whether Hypothesis 3.2 holds in the given network using polynomial algorithms.

The graph in Figure $3.16 \mathrm{~b}$ consists of 5 different SCCs. These are also depicted in the original network of Figure 3.16a. Note that SCC $G_{1}$ and $G_{4}$ are violating Hypothesis 3.2, since in the former a conflict is present in a feedback, and in the latter there is self-loop to a conflict.

Now we can state the idea for the ordering in the original network, that we were looking for at the beginning of the section. First we need to compute the graph of relations among conflicts (using which, one can also verify Hypotheses 3.1 and 3.2), then we have to find all the SCCs of this graph, which forms a directed acyclic graph (DAG), with each SCC as its nodes. Now performing a topological sort on this DAG, we have an ordering over different sub-networks of the original network. For the example given in Figure 3.16, there are several possible ordering, for instance:

- $\left(G_{1}, G_{3}, G_{2}, G_{4}, G_{5}\right)$ or

- $\left(G_{3}, G_{1}, G_{2}, G_{5}, G_{4}\right)$.

Now we can apply rate adaptation on these ordered components, one after the other. We can do this, since the data needed for conflict resolution in each SCC, is completely determined by SCCs in its upper stream, i.e., those that appear earlier in the topological sort. Note that any of the possible orderings can be used for rate adaptation. For instance, in the example given in Figure 3.16, what is important is that rate adaptation for $G_{1}$ should be done before $G_{2}$, and the rates in $G_{2}$ should calculated before $G_{4}$, and $G_{5}$. This idea is formalized in the Algorithm 3.4, which is the analogous version of Algorithm 3.3, with the difference that it can handle multiple sharing.

In lines 1-3 the above explained process for ordering SCCs in the graph of relations among conflicts, is performed. In line 4-8 we iterate over the ordered set of SCCs. In line 5 it is checked whether this SCC contains an actual conflict for which sharing should be used for resolution. If not, rate adaptation using priorities can be performed (Section 3.5.1). If there is a conflict structure with sharing (line 7-8), then we have to use the flow sharing algorithm, given in Algorithm 3.2. Note that because of Proposition 3.1, this SCC contains only one vertex, which is associated with all the output transitions connected to the continuous place $P^{c}$. Therefore, invoking ShareFlow on this place is enough to adapt the rates of all transitions in this SCC. 


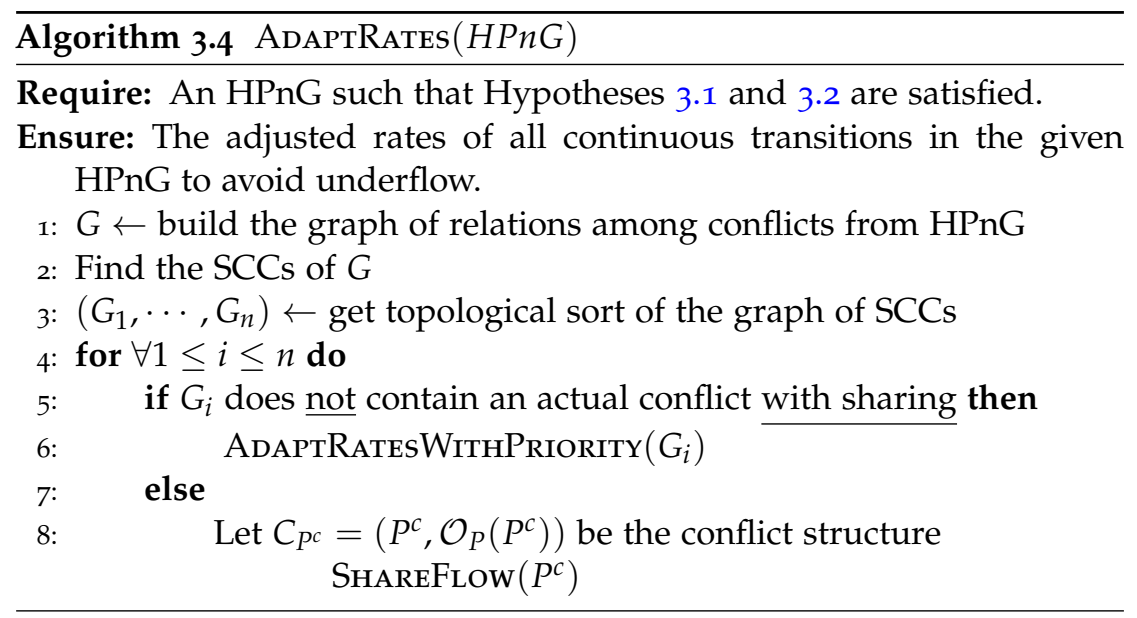

Note that in the above algorithm, feedback in general is allowed. In case a conflict structure with sharing is involved in a feedback, i.e., Hypothesis 3.2 is violated, the designer can overcome this problem by resolving the conflict using priority instead of sharing.

\subsection{RELATED WORK}

There are several formalism of Petri nets that include stochasticity in one way or the other. Each of these formalism are imposing a limitation on a certain aspect of the model, tailoring it toward a specific application area. Stochastic Petri nets (SPN) introduced in [29], are built upon of discrete Petri nets and do not allow continuous places and transitions. In SPNs each transition is associated with an exponential distribution, therefore due to the memmoryless property, they are equivalent to continuous time Markov processes [30]. Generalized Stochastic Petri nets (GSPN) [21,28] are an extension of SPNs by allowing immediate transition. GSPNs are also shown to be equivalent to Markovian models.

In order to include non-exponential distributions Markov regenerative Stochatic Petri nets (MRSPNs), and the method of supplementary variables $[23,32]$ are introduced. The idea of the supplementary variable method is to include age variables into the state description which represent the time since enabling of the transitions with generally distributed firing times (similar to the concepts of clocks in the contexts of HPnGs). Thus, a mixed discrete and continuous state space is defined. Note that continuity in this state space description is related to passage of time, and not the content of continuous places.

Fluid Stochastic Petri nets (FSPNs) introduced in $[16,24]$ are an extension of stochastic Petri nets with continuous places and transitions. The solution techniques for this model are based on solving differential 
Table 3.3.: Comparison of different formalism and solution methods of Petri net for handling continuity and stochasticity.

\begin{tabular}{|c|c|c|}
\hline Models & \# Continuous places & Stochasticity \\
\hline SPN, GSPN [21,28-31] & No & Only exponential \\
\hline $\begin{array}{c}\text { MRSPN, Method of supplementary } \\
\text { variables }[22,23,32,33]\end{array}$ & No & Arbitrary \\
\hline FSPN $[16,24,27]$ & Yes, but limited & Only exponential \\
\hline $\begin{array}{l}\text { FSPN with method of supplementary } \\
\text { variables [25] }\end{array}$ & Yes, but limited & Arbitrary \\
\hline
\end{tabular}

equations regarding the underlying stochastic process, hence, because of intrinsic limitations of such solution methods the number of continuous places in these models are limited. Numerical methods based on simulation are proposed in [27] to overcome this limitation. Moreover, [25] has tackled the inadequacy of exponential distributions, by using the supplementary variable method for the age variables of transitions with generally distributed firing times, and also allowing normally distributed rates for continuous transitions. The latter means that continuous places can be filled and emptied with randomly chosen rates from a normal distribution. However, the solution method in this work is limited to deriving state equation which are again in need of numerical methods, hence exactness is lost.

Table 3.3 summarizes the different approaches and solution methods using Petri nets formalism, based on their ability to support continuity and stochasticity.

The HPnG model proposed in this chapter is intended to overcome two limitations:

1. removing the constraints on the number of continuous places, such that still efficient analytical methods are possible,

2. overcoming the insufficiency of exponential distribution, by allowing general transitions with arbitrary probability distributions. 


\section{4}

STATE SPACE AND LOGIC

The content of this chapter is based on:

[51] H. Ghasemieh, A. Remke, B.R. Haverkort, and M Gribaudo. Region-based analysis of hybrid Petri nets with a single general one-shot transition. In Formal Modeling and Analysis of Timed Systems, volume 7595 of LNCS, pages 139-154. Springer, 2012

[52] H. Ghasemieh, A. Remke, and B. R. Haverkort. Survivability evaluation of fluid critical infrastructures using hybrid Petri nets. In 19th Pacific Rim International Symposium on Dependable Computing, pages 152-161. IEEE, 2013.

In this chapter we introduce the main idea, which serves as the backbone of most of the methods being introduced in this thesis. We provide a graphical representation of the state space of HPnGs, the so-called Stochastic Time Diagrams (STD). The evolution of an HPnG can be represented by an STD that illustrates the system state at each time conditioned on the firing time of general transitions.

In Section 4.1 we will first intuitively explore the idea of STDs as state representation using a simple example for the case of only one stochastic variable. After this, we formalize the important concept of partitioning of state space to regions, and will discus briefly how these regions could be employed to compute some measures of interest. Moreover, Section 4.2 introduces and formalizes the Stochastic Time Logic (STL) which will be used to express measures of interest about HPnGs. Finally, in Section 4.3 we conclude by reviewing the related works to the content of this chapter.

\subsection{STOCHASTIC TIME DIAGRAMS}

In this section we first introduce the idea of STD for the case when only one general transition fires only once, i.e., when there is only one active stochastic variable. Later, in Subsection 4.1.2, we will formalize this idea for the case of multiple stochastic variables. 


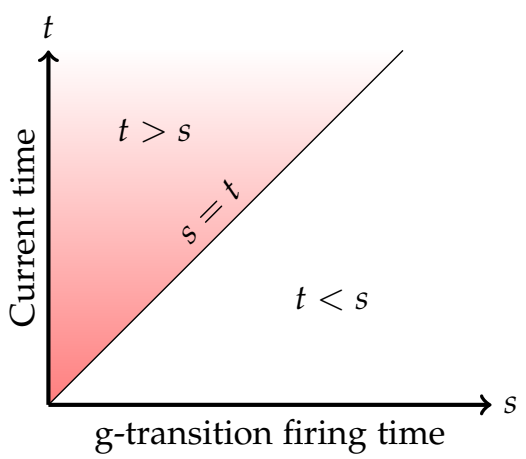

Figure 4.1.: Generic presentation of STD.

\subsubsection{One Stochastic Variable}

Given an initial state of an HPnG and a predefined value for the firing time of the general transition (denoted $s$ ) the state of the system can be determined for all the future times $t$ starting from a given initial state. Hence, in order to characterize the system state, we consider a two-dimensional diagram with time $t$ on the vertical axis and the values (realizations) of stochastic variable $s$ on the horizontal axis. Each point in this diagram is associated with a unique system state. A generic version of this diagram is shown in Figure 4.1. The area above the line $t=s$ contains all states for which we assume that the general transition has fired already, i.e., the current system time $t$, is larger than the firing time $s$ of the general transition, $t>s$. The area below the line $t=s$, in contrast, represents all states where the general transition has not fired yet, i.e., $t<s$. In this area the evolution of the system is independent of parameter $s$.

The idea behind this state space representation, is that instead of dealing with infinitely many points in the ts-plane, we can partition it into several regions, such that all points inside one region can be associated with the same system state. These regions exist, because the state of the system does not change until an event occurs, e.g., a continuous place reaching its lower/upper boundary or an enabled transition fires. So, an event imposes a change in the state of the system. We demonstrate these concepts using an example in the following.

\section{Reservoir Example}

In order to illustrate the mentioned concepts, we construct the STD for the reservoir example given in Section 3.3.1. This example is repeated in Figure 4.2. We start with the initial condition that the reservoir $P_{m}$ is 
empty, and the places $P_{p}$ and $P_{d}$ each contain a token, hence, the pumps are working.

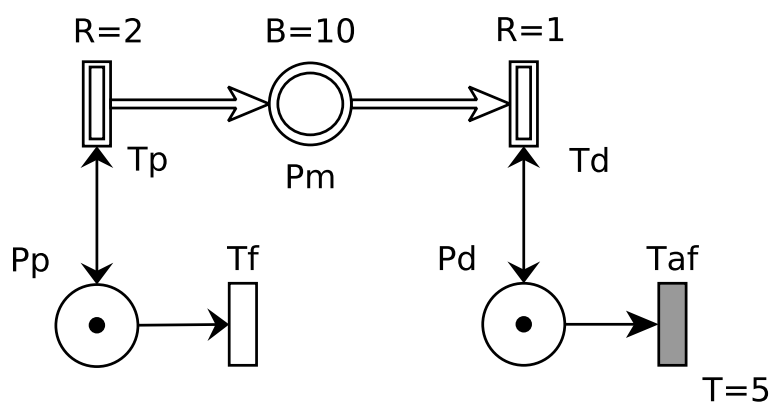

Figure 4.2.: Reservoir model.

Assuming that the general transition has not fired, i.e., $s>t$, there are two possible events: either transition $T_{a f}$ fires at time 5 or reservoir $P_{m}$ reaches its upper boundary. Since the overall rate of change (drift) of fluid into $P_{m}$ is 1 (the difference of rates of $T_{d}$ and $T_{p}$ ) in this sense, it takes 10 time units to become full. So the first event to occur is $T_{a f}$, firing at time 5. Figure 4.3a illustrates the occurrences of these events, by two horizontal lines $t=5$ and $t=10$, relating to the firing of $T_{a f}$, and to $P_{m}$ reaching its upper boundary, respectively. The line associated with the firing of $T_{a f}$ is indicated in blue as it is the event which is going to happen first. After this, since transition $T_{d}$ is no longer enabled, due to the firing of $T_{a f}$, the token in $P_{d}$ is removed, and the drift at $P_{m}$ becomes 2. Since the reservoir contains 5 units of fluid at time $t=5$, it takes 2.5 time units to reach its upper boundary (it takes 2.5 time units to fill in the remaining 5 units capacity with rate 2 ). Therefore, this event occurs at time $t=5+2.5=7.5$. In Figure $4.3 \mathrm{~b}$, this is shown by line $t=7.5$, labeled $P_{m}$. After entering the area above this line, no other event is possible anymore, i.e., we reach an absorbing region.

After partitioning the area below the line $t=s$, this line is divided into three segments, as is illustrated in Figure $4.3 \mathrm{~b}$. Then, in order to partition the area above the line $t=s$, the area above each of these segments has to be considered separately since the marking of the system is different below each of them. Starting from the initial state of the system, first consider that the general transition fires at time $s \in[0,5)$. When the general transition fires, i.e., the system passes the line $t=s$, two events are possible: either $T_{a f}$ fires or $P_{m}$ reaches its lower boundary. Before the general transition fired, place $P_{m}$ had drift 1 and since $s$ time units are elapsed, it now contains $s$ units of fluid. After the general transition has fired, the transition $T_{p}$ is disabled, and the drift at $P_{m}$ becomes -1 . 


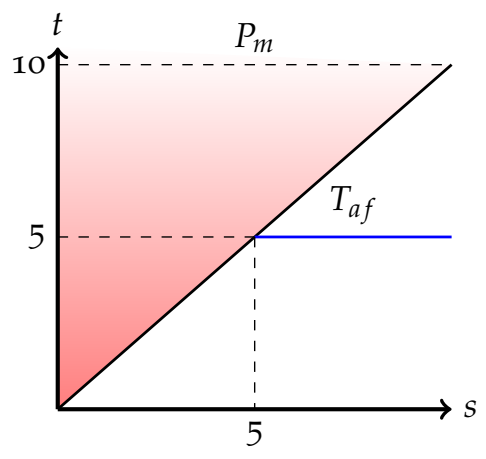

(a)

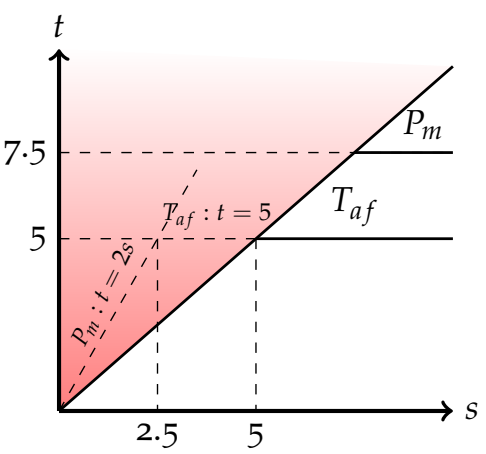

(c)

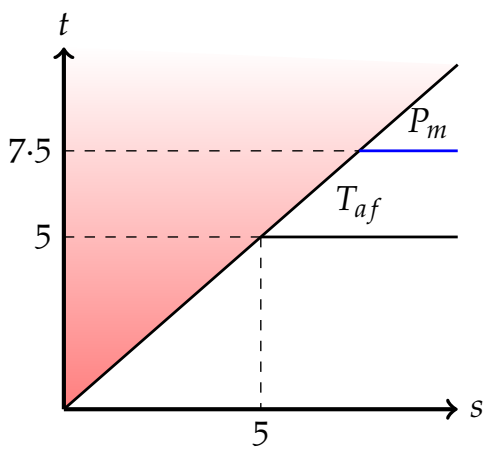

(b)

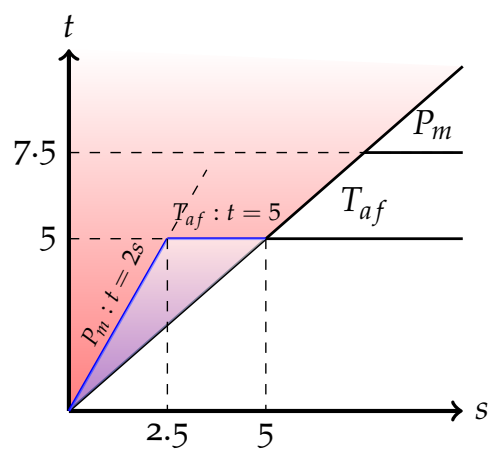

(d)

Figure 4.3.: Formation of the region over the interval $[0,5)$.

Now, either the reservoir becomes empty or the deterministic transition fires, which stops the demand. To find out which of these events is going to occur first, we have to compare their occurrence times, which may depend on $s$. Let $\Delta t$ be the time needed for $P_{m}$ to become empty, we have: $\Delta t=s$. The previous event has occurred at time $s$, so the reservoir becomes empty at $t=\Delta t+s=2 s$. Since transition $T_{a f}$ fires at time 5 , the occurrence time equation of this event is simply $t=5$ and does not depend on $s$. These two possible events are illustrated in Figure $4.3 \mathrm{c}$. The minimum of both equations then determines the next event, as shown in the shaded area in Figure $4.3 \mathrm{C}$, by the blue segment. The procedure forms a region, i.e., a set of $(s, t)$ points, over the segment $t=s$ for $s \in[0,5)$. We will formalize the definition of regions in the next section.

Note that each edge of this region represents the occurrence time of an event, hence, the procedure can be repeated recursively for each of them, i.e., we can form another region over each edge, and continue this procedure until we have obtained the complete partitioning of the 


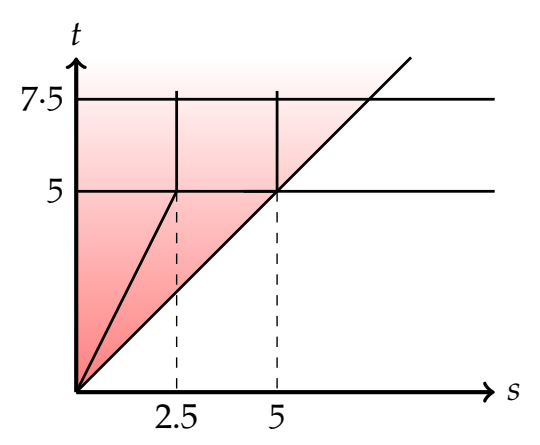

Figure 4.4.: Formation of the region over the interval $[0,5)$.

stochastic area, up to the maximum analysis time. Figure 4.4 shows the complete STD for the reservoir example with nine different regions.

The partitioning of the state space into regions, i.e., the shape of the STD as in Figure 4.4, depends on the structure of the model at hand. These regions exist, because the state of the system does not change until an event occurs. Therefore, the boundary between two regions represents the occurrence of an event. We will later prove that for a given HPnG all boundaries are straight lines.

\section{A glimpse into later computations}

Recall that each general transition fires according to the probability density function $g(s)$. To compute measures of interest for HPnGs, the state space needs to be deconditioned according to $g(s)$. In order to compute the probability to be in a specific system state at time $\tau$, it suffices to find all regions intersecting the line $t=\tau$ that correspond to the specific system state and integrate $g(s)$ over the intersection. This idea is illustrated for a given partitioning in Figure 4.5. As can be seen three regions are intersecting with the line $t=\tau$, and in one of them (the one on the left) a given system property holds; and the probability of holding that property is shown by the red area beneath the shown PDF $g(s)$. We will later explore this idea more formally in Chapter 5 .

\subsubsection{Multiple Stochastic variables: formalization}

In this section we formalize the idea of partitioning the STD into regions for an arbitrary number of stochastic variables. Recall from Section 3.2.2, that the firing of each general transition $T_{i}^{d}$ introduces a random variable denoted by $S_{i} \in \mathcal{S}_{i}$. Moreover, as mentioned earlier, we assume prior to the analysis that the number of these firings are known (by using isolated places connected to general transitions, see Figure 3.2). Let $n$ be the total number of firings of general transitions, and $\mathbf{S}=\left(S_{1}, \cdots, S_{n}\right) \in$ 


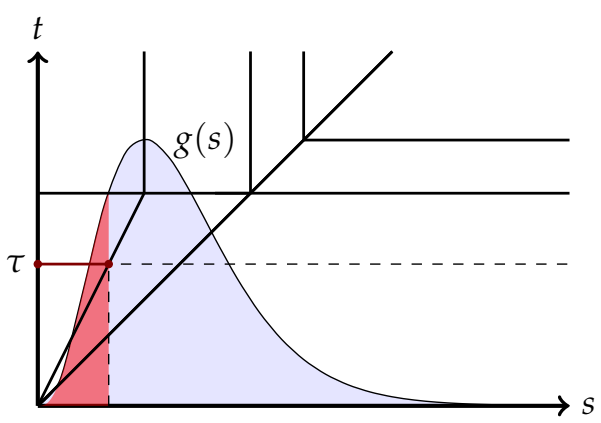

Figure 4.5.: Deconditioning according to the probability density function $g(s)$.

$\mathcal{S}_{1} \times \cdots \times \mathcal{S}_{n}=\mathcal{S}$ be the vector of $n$ random variables, representing the firing times of the general transitions.

We should note that support of stochastic variables could be dependent on the value of other stochastic variables. This may happen when firing of a general transition may enable or disable the other general transitions. For instance in the example given in Figure 4.6, $T_{2}$ is only enabled after $T_{1}$ has fired, and $T_{4}$ is enabled only if $T_{2}$ and $T_{3}$ both have fired. Therefore, the potential supports of the stochastic variables are as follows:

$$
\mathcal{S}_{1}=[0, \infty), \mathcal{S}_{2}=\left[S_{1}, \infty\right), \mathcal{S}_{3}=[0, \infty), \mathcal{S}_{4}=\left[\max \left\{S_{2}, S_{3}\right\}, \infty\right) .{ }^{1}
$$

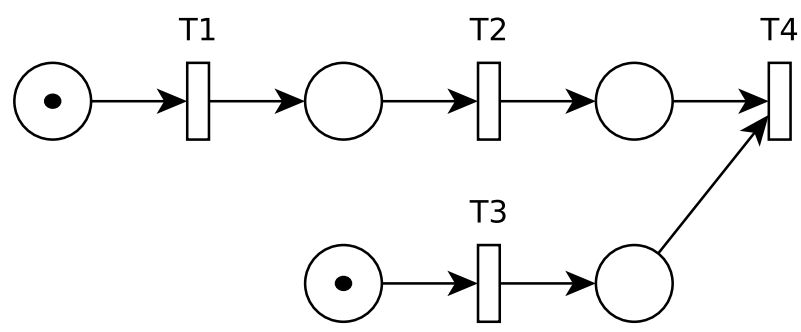

Figure 4.6.: Demonstration of firing time dependence of general transitions on each other.

Addition of each stochastic variable to the system will add one dimension to the STD. If $n$ stochastic variables are present in a system, the

1 Note that determining the support of stochastic variables in general, needs a more involved treatment. However, we do not discus this, since at the end our exact algorithm is going to be limited in number of stochastic variables. 
STD will have $n+1$ dimensions, $n$ of which are associated with stochastic variables and the $(n+1)$ th dimension is associated with the time $t$. Each point in the STD is associated with a unique HPnG system state, denoted by $\Gamma(\mathbf{s}, t)$, which is defined in Section 3.2.3. Figure 4.7 illustrates a generic STD with two stochastic variables.

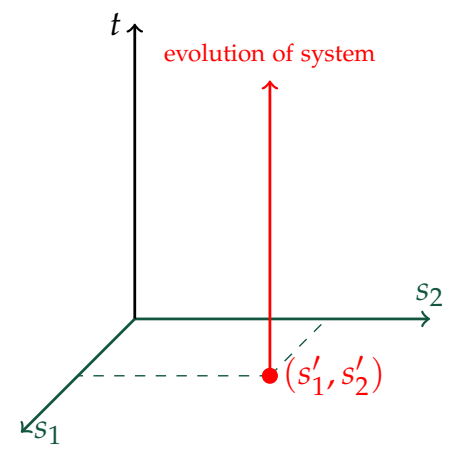

Figure 4.7.: Generic presentation of STD in 3 dimensions.

As mentioned earlier, the idea is to partition the infinitely many points in the $t s$-space, into several regions. These regions exist, because the state of the system does not change until a so-called event occurs. In each system state, three types of potential events can occur:

(i) a continuous place reaching its lower/upper boundary,

(ii) a continuous place content reaches the threshold of a connected guard arc,

(iii) an enabled transition, either deterministic or general, fires.

Event type (i) imposes a change in the drift of the continuous place, due to rate adaptation, as described in Section 3.5, and event type (ii) will enable or disable a transition. In case of an immediate transition, it will fire and alter the discrete marking immediately, and if it is a deterministic transition its clock drift will be set to one, thereby changing a continuous variable. Finally, event type (iii) alters the discrete marking $\mathbf{m}$, or the general transitions vector $\mathbf{g}$. In any case, an event may cause a change in the discrete marking, a change in drift (either for clocks or fluid levels) or a change in the vector indicating the number of firings of each general transition.

We define a region as a maximal set of states that while system reside in it no event occurs, i.e., discrete marking, drift of continuous variables and general transitions vector remain unchanged within a region. Moreover, by the occurrence of an event the system enters another region. This leads to the following definition. 
Definition 4.1. A region $\mathcal{R}$ is a maximal connected set of $(\mathbf{s}, t)$ points in a given $S T D$, for which we have:

$$
\forall\left(\mathbf{s}_{1}, t_{1}\right),\left(\mathbf{s}_{2}, t_{2}\right) \in \mathcal{R}:\left\{\begin{array}{l}
\Gamma\left(\mathbf{s}_{1}, t_{1}\right) \cdot \mathbf{m}=\Gamma\left(\mathbf{s}_{2}, t_{2}\right) \cdot \mathbf{m}, \\
\Gamma\left(\mathbf{s}_{1}, t_{1}\right) \cdot \mathbf{d}=\Gamma\left(\mathbf{s}_{2}, t_{2}\right) \cdot \mathbf{d}, \\
\Gamma\left(\mathbf{s}_{1}, t_{1}\right) \cdot \mathbf{g}=\Gamma\left(\mathbf{s}_{2}, t_{2}\right) \cdot \mathbf{g} .
\end{array}\right.
$$

Note that vector $\mathbf{d}$ contains both drifts of continuous places and clocks for discrete transitions, as defined in Section 3.2.3. The reason for this is that because of guard arcs, a discrete transition can be enabled or disabled for the same discrete marking, due to a change in the continuous marking. This is an event type (ii), hence, represents a move to another region.

The shape of the regions depends on the structure and the parameters of the model at hand. However, inside a region all continuous variables, i.e., the amount of fluid and the clock values, can be represented by simple linear equations in s and $t$. Intuitively, this is because in a region, all continuous variables clocks or continuous places contents, are associated with a constant drift, as shown in Corollary 3.1. Using this, we infer that the boundaries between regions, which represent the occurrence of an event, are characterized by linear functions of $\mathbf{s}$ and $t$, which represents a hyperplane in $n+1$ dimensions. Hence, each region in the STD can be considered a polyhedron, in $n+1$ dimensions. These are later explored in detail in Chapter 5.

\subsection{STOCHASTIC TIME LOGIC}

In this section we introduce the logic for expressing properties and measures of interest, at a certain time. The logic is called Stochastic Time Logic (STL); it is used to reason about the underlying state space of an HPnG, namely the STD. As an STD explicitly takes into account all possible values of $\mathbf{s}$ and $t$, hence, with the proposed logic it is possible to reason whether an STL formula holds for a certain system state $\Gamma(\mathbf{s}, t)$. However, note that STL reasons on the deconditioned state-space of an HPnG, i.e., on the regions of an STD, which does not yet take into account the distribution of general transitions. To lift this to the level of the HPnG we also introduce a probability operator that explicitly takes into account the density function of the general transitions.

An STL formula is divided in two main categories: state-based and Until formula. The former reason about holding of a certain property at a given time, and the latter, expresses the properties to hold during passages of time, using the Until operator. 
Definition 4.2 (Stochastic Time Logic). An STL formula $\Psi$ is defined as

$$
\begin{aligned}
& \Psi:=\Theta \mid \Omega \\
& \Theta:=\mathrm{tt}\left|x_{P} \geq c\right| m_{P}=a|\neg \Theta| \Theta \wedge \Theta, \\
& \Omega:=\Theta \mathcal{U}^{\left[T_{1}, T_{2}\right)} \Theta
\end{aligned}
$$

(State-based)

(Until)

where $T_{1}, T_{2} \in \mathbb{Q}^{+}\left(T_{2}>T_{1}\right), x \geq c$ and $m=a$, with $a \in \mathbb{N}, c \in \mathbb{R}^{+}$, are called continuous and discrete atomic properties, respectively.

Note that, the above definition does not allow nesting of Until formulas, i.e., nested Until operators are not allowed. In the following we define two different satisfaction relations,

(i) $\models \mathbf{s}, t$ between a single system state $\Gamma(\mathbf{s}, t)$ and an STL formula $\Psi$,

(ii) $\models{ }^{t}$ between a subset of $\mathcal{S}$, i.e., the Cartesian product of the support of stochastic variables as introduced in Section 4.1.2, and an STL formula $\Psi$.

The satisfaction relation $\models$ s,t takes into account a single point in the STD, which corresponds to a single system state. The superscript "s, $t$ " is to emphasis that this relation is defined over a system state for a specific values of vector $\mathbf{s}$, and time $t$. This satisfaction relation is defined as follows:

Definition 4.3 (Satisfaction on system states).

$$
\begin{array}{ll}
\Gamma(s, t) \models s, t & \forall t, s, \\
\Gamma(s, t) \mid \models^{s, t} m_{P}=a & \text { iff } \Gamma(s, t) \cdot m_{P}=a, \\
\Gamma(s, t) \models{ }^{s, t} x_{P} \geq c & \text { iff } \Gamma(s, t) \cdot x_{P} \geq c, \\
\Gamma(s, t) \models{ }^{s, t} \neg \Theta & \text { iff } \Gamma(s, t) \not \Theta, \\
\Gamma(s, t) \models{ }^{s, t} \Theta_{1} \wedge \Theta_{2} & \text { iff } \Gamma(s, t) \models \Theta_{1} \wedge \Gamma(s, t) \models \Theta_{2}, \\
\Gamma(s, t) \mid{ }^{s, t} \Theta_{1} \mathcal{U}^{\left[T_{1}, T_{2}\right]} \Theta_{2} & \text { iff } \exists \tau \in\left[t+T_{1}, t+T_{2}\right]: \\
& \Gamma(s, \tau) \models{ }^{s, t} \Theta_{2} \wedge\left(\forall \tau^{\prime} \in[t, \tau]: \Gamma\left(\tau^{\prime}, s\right) \models{ }^{s, t} \Theta_{1}\right) .
\end{array}
$$

The first 5 items in the definition are self explanatory. For the last item, i.e., the STL until operator $\Theta_{1} \mathcal{U}^{\left[T_{1}, T_{2}\right]} \Theta_{2}$ and a system state $\Gamma(\mathbf{s}, t)$ we have to check, whether starting from time $t$ and for a given sample $\mathbf{s}$, the evolution of the system is such that a time point $\tau$ exists at which $\Theta_{2}$ holds and before which $\Theta_{1}$ holds. Note that for a given system state $\Gamma(\mathbf{s}, t)$ the evolution over time is deterministic and coincides with a vertical line in the STD, starting at point $(\mathbf{s}, t)$. Hence the analysis of the STL until operator for a given system state boils down to checking, whether this line only intersects with regions where $\Theta_{1}$ holds until a region is hit where $\Theta_{2}$ holds within the defined time interval. This is discussed in depth in Section 6.2. 
As mentioned earlier, STD combines system states with the same characteristics into regions. This can be exploited for checking sets of states instead of individual system states. Hence, we introduce a satisfaction relation $\models^{t}$ over subsets of the joint support of stochastic variables, $\mathcal{S}$. The superscript " $t$ " is added to stress the fact that this satisfaction relation depends on a given time $t$. This allows for more efficient model checking procedures than checking each system state individually.

Definition 4.4. Let $A \subseteq \mathcal{S}$, and $\Psi$ an STL formula:

$$
A \models{ }^{t} \Psi \quad \text { iff } \forall s \in A: \Gamma(s, t) \models{ }^{s, t} \Psi .
$$

Definition 4.5. The satisfaction set of $\Psi$ at time $t$, denoted $S a t^{t}(\Psi)$, is defined as,

$$
\begin{aligned}
\operatorname{Sat}^{t}(\Psi) & =\left\{s \in \mathcal{S}: \Gamma(s, t) \models^{s, t} \Psi\right\} \\
& =\left\{A \subseteq \mathcal{S}: A \models^{t} \Psi\right\} .
\end{aligned}
$$

The second equation in the definition is for the cases when the satisfaction set can be decomposed into a family of connected sets in $\mathcal{S}$. We call these connected sets areas. As we will see later, this decomposition is particularly useful for computational reposes.

While the explicit dependency on s (or sets of s-values) is used for the efficient computation of properties, in the end we want to know whether a given STL formula holds at time $t$ for the HPnG model of interest with a certain probability. Hence, we introduce a probability operator $\mathbb{P}_{\sim p}(\Psi)$ which is wrapped around an STL formula, where $p \in[0,1]$ is a real number and $\sim \in\{\leq,<,>, \geq\}$ a comparison operator. It abstracts from the possible values of $\mathbf{s}$ by deconditioning with the joint CDF $G(\mathbf{s})$ , as follows.

Definition 4.6. Let $\Gamma(t)=\{\Gamma(s, t) \mid s \geq 0\}$ be the set of possible system states at time $t$, then the satisfaction relation for the probability operator $\mathbb{P}_{\sim p}$ is defined as:

$$
\Gamma(t) \models \mathbb{P}_{\sim p}(\Psi) \quad \text { iff } \operatorname{Prob}^{t}(\Psi) \sim p,
$$

where

$$
\begin{aligned}
\operatorname{Prob}^{t}(\Psi) & =\int_{\mathcal{S}} \mathbb{1}_{\psi}(\Gamma(s, t)) d G(s) \\
& =\int_{\text {Sat }^{t}(\Psi)} d G(s)=\sum_{A \in \operatorname{Sat}^{t}(\Psi)} \int_{A} d G(s) .
\end{aligned}
$$

Note that in the last term in the above integration, a decomposition of the satisfaction set into areas, mentioned in Definition 4.5, is used. This indeed makes the computations possible using standard integration tools. Moreover, one can exclude null sets, i.e., sets of measure zero, like singular values, as they are not contributing to the final integration. We will elaborate on the efficient computation of the above integration in Chapter 6. 


\section{$4 \cdot 3$ RELATED WORK}

\section{State space partitioning}

The idea of a partitioning the underlying state-space of hybrid systems is not new, e.g., the underlying state-space of Timed Automata (TA) $[18,53]$ can be partitioned into zones, where each zone represents a symbolic system state. However, due to the fact that all real-valued clocks have an identical drift of 1 the shape of a zone is restricted comparing to the shape of the regions resulting for HPnGs. Similarly, a partitioning of the state-space of hybrid systems has been introduced in [17]. Also for Dense-Time Reactive Systems [54,55] that are a generalization of Timed Petri Nets [56] a partitioning of the state space into state classes has been introduced. Although, such systems do not include continuous variables they allow to equip timed transitions with an interval indicating their firing time. Again, since time evolves linearly with derivative 1, the shape of state classes is similarly restricted as zones for TA.

Dynamical Systems having Piecewise-Constant Derivatives (PCDs) [57] represent a class of hybrid systems where the evolution of the continuous variables is piecewise-linear and the control component of a state is fully determined by the values of the continuous variables. This also results in a set of regions, where each region is associated with a constant vector which identifies the rates at which the continuous variables evolve in time. Similar to HPnGs, PCDs allow different slopes for the continuous variables within one region and more general guards for discrete transitions. However, in contrast to PCDs, the discrete state of an HPnG is not fully described by the values of the continuous variables (due to rate adaptation of continuous transitions), hence, allows for a more general discrete component.

An important comment here is in order. In all the mentioned approaches above, the partitioning of state space is done according to the values of the continuous variables. In contrast, in STDs the partitioning is done over the time and the support of stochastic variables.

\section{Logics}

Algorithmic verification of reactive systems ${ }^{2}$ is centered around modelchecking of temporal logics. This includes both discrete and dense time systems. Linear Temporal logic (LTL) [59], and Computation Tree Logic (CTL) [6o] are both used to express properties regarding systems with discrete timing. LTL, as its name suggests, considers a linear notion of time, which means that at each moment there is a single successor moment. On the other hand, CTL is used for the branching nature of

2 A reactive (responsive) system is known as a system which maintains a continuous interaction with its environment [58]. 
time, where each moment may be followed by different successors, i.e., a tree-like structure of time is assumed.

The logic STL, introduced in this chapter, mostly resembles LTL, since no branching in the course of time is involved. This is because once the values of stochastic variables are fixed, the evolution of the system is deterministic. This can be seen as a fixed path on which linear temporal properties can be investigated. However, we should emphasis that for HPnGs, a continuous time evolution is involved, i.e., we are dealing with a dense time system, instead of discrete jumps among states of a transitions system.

It is also important to note that the satisfaction sets for both LTL and CTL are defined over the states of a transition system under consideration. However, for STL a satisfaction set is defined over a set of realizations of values of stochastic variables. This means the verification is done by providing the set of values of stochastic variables, for which the given STL formula is satisfied over the course of time.

Moreover, note that although we have decomposed the definition of STL into two categories (of state-based and until formulas), this should not be confused with the state- and path-based formulas used in CTL. The main reason for this decomposition of STL is to avoid nesting of Until formula. Moreover, we will treat state-based and Until formulas differently, since they need different model checking algorithms.

The Metric Interval Temporal Logic (MITL) $[61,62]$ is a linear temporal logic that is interpreted over timed state sequences. MITL is used for verification of timing properties of real-time systems with a dense semantics. A standard way of introducing a real dense time into the syntax of temporal languages is to constrain the temporal operators, such as Until, with time intervals. This is similar to the approach we have taken in the definition of STL.

MITL uses a linear or trace semantics for reactive systems. The linear semantics of a system is a set of possible behaviours, each of which is represented by a sequence of system states. This naturally incorporates real time by associating every state with a time interval, indicating the period during which the system is in that state. The possible behaviours of a real-time system are represented by such timed state sequences. The approach we took in defining the semantics of STL is similar, because when we condition the system on specific values of stochastic variables, the result is a deterministic hybrid Petri net, which is known to be reducible to a hybrid automata [34].

Since we are also considering a system with dense time semantics, STL resembles MITL in its expressiveness of properties. Also note that, STL like other time dense logics, e.g., MITL, and unlike discrete time logics, e.g., LTL and CTL, does not allow the next operator.

As a final remark we should note that STL is verified at a specific time instance $t$. This together with the assumption that the Until operator is 
always bounded by a time interval, certifies that the verification process is always done for a finite time horizon. Therefore, the undecidability results regarding verification of hybrid systems do not apply here. Moreover, the use of bounded temporal properties is justified from the pragmatic point of view where the behaviour of a system is observed for a finite time interval. 

Part II

ANALYSIS AND ALGORITHMS 



\section{EFFICIENT STATE SPACE GENERATION}

The content of this chapter is based on:

[51] H. Ghasemieh, A. Remke, B.R. Haverkort, and M Gribaudo. Region-based analysis of hybrid Petri nets with a single general one-shot transition. In Formal Modeling and Analysis of Timed Systems, volume 7595 of LNCS, pages 139-154. Springer, 2012

[63] H. Ghasemieh, A. Remke, and B. R. Haverkort. Hybrid Petri nets with multiple stochastic transition firings. In 8th International Conference on Performance Evaluation Methodologies and Tools, pages 217-224. ICST, 2014.

This chapter establishes the algorithms for the generation of the state space, i.e., the Stochastic Timed Diagram (STD), as introduced in Chapter 4 . The main challenge in generation of STDs is grouping points in the $(\mathbf{s}, t)$-space into regions. This becomes more demanding when we allow multiple stochastic variables, because as mentioned in Section 4.1.2, addition of each stochastic variable adds to the dimensionality of STD.

In this chapter we first discuss the STD generation algorithm for the case of one general one-shot transition, i.e., a general transition which can fire only once. This means we assume there is only one active stochastic variable present in the system during the time of analysis. We follow this approach because dealing with only one stochastic variable makes the explanation, hence, grasping the essence of the algorithms for generation of STD, much easier. Moreover, it provides the basis for the generation of STDs with multiple stochastic variables.

The proposed method in this chapter for the generation of state space highly depends on algorithms from computational geometry, especially for the case of multiple stochastic variables. Specifically, the generation of the STD is reduced to two well-known problems: half space intersection [64] and hyperplane arrangement [65]. For both of these problems feasible algorithms and solutions exist. However, to the best of our knowledge, there is no implementation of hyperplane arrangement for more than 
two dimensions, which corresponds to the presence of two stochastic variables in our case.

For generation of STDs in this chapter two important comments are in place. Firstly, for conflict resolution of discrete transitions, we use prioritizing as introduced in Section 3.4. This ensures that the only probabilistic behaviour in the analysis of HPnGs is due to firing of general transitions. Secondly, the generation of the STDs, are being done up to a maximum time, to which we refer as the maximum time of analysis. This time is usually determined by the the time at which we want to verify whether a certain property, i.e., STL formula is satisfied.

This chapter is organized as follows. First we introduce and formalize the essence of the state space generation for the case of only one stochastic variable in Section 5.1. After this, the formalism is extended to the case when multiple stochastic variables are present in Section 5.2. Moreover, in this section we discus the complexity and limitation of this generation method for multiple stochastic variables. Finally, in Section 5.3, we briefly discuss the predecessor method to the generation of STDs.

\subsection{ONE STOCHASTIC VARIABLE}

This section should be considered as a prelude to Section 5.2, since it considers the special case of having only one firing of a general transition. Therefore, in this section we refer to some algorithms which are going to be discussed in the next section. However, these algorithms are easy to understand intuitively for the case of one stochastic variable. We will also leave the complexity analysis, and the discussion of finiteness of algorithms for the general case, which is going to be treated in Section 5.2.

In this section we present the algorithm for the generation of the STD in two main phases: when the general transition has not fired and when it has fired. We follow this approach because it simplifies the explanation and shows how the firing of a general transition (hence, the existence of a stochastic variable) will influence the structure of the STD.

Recall from Chapter 4 that the idea is to parametrize the firing time of the general transition, i.e., the value of stochastic variable. Since in this section we assume that there is only one stochastic variable we denote its value by $s$. Moreover, let $t$ be the current time of the analysis. When the general transition has not fired yet, we have $t<s$, and when it has fired we have $t \geq s$. Therefore the line $t=s$ separates the two areas for partitioning as shown in Figure 4.1 in Chapter 4 (and also in Figure 5.1 in the following section). 


\subsubsection{General transition has not fired yet}

Since the assumption is that the general transition has not fired yet, in this phase the evolution of the system is purely deterministic. In other words, while $s>t$ the occurrence time of events does not depend on $s$, i.e., by changing $s$ the shape of regions do not change. Therefore, the regions, partitioning the area below the line $t=s$, are constructed with lines parallel to the s-axis, as shown in Figure 5.1. This means each region $R_{i}$ is determined uniquely by the time at which the region is entered and and the time it is exited. So, each of these regions are associated with an interval $\left[h_{i}, h_{i+1}\right)$, where $h_{i}$ is the occurrence time of the event that changes the state of the system from region $R_{i-1}$ into region $R_{i}$, and $h_{i+1}$ is the occurrence time of the event that causes the system to go from region $R_{i}$ to $R_{i+1}$. We refer to these regions as deterministic regions. These regions and their corresponding intervals on the $t$-axis are shown on Figure 5.1.

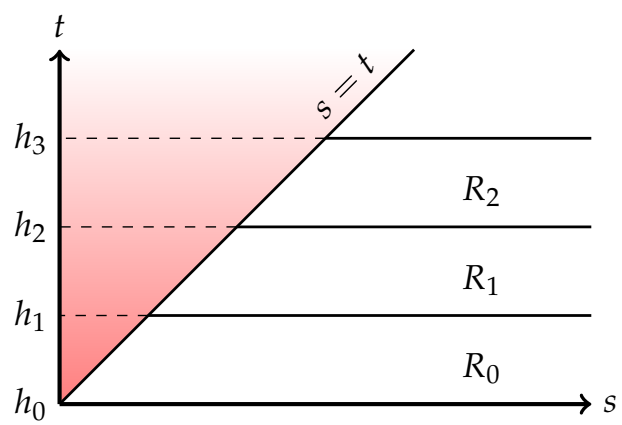

Figure 5.1.: Deterministic regions.

In order to partition the area below the line $t=s$, one needs to determine the next event at each time point, update the marking of the HPnG after the occurrence of that event, advance the time to that point, and continue this process until reaching the maximum time of analysis. Recall from Section 4.1.2, that during the evolution of the system at each point, three types of events are possible: an enabled discrete transition reaches its firing time and fires, or a continuous place reaches either its boundaries or threshold of a guard arc connected to it. So, for all continuous places we need to find the time at which they may reach a lower or an upper boundary. These boundaries are either specified by 
the capacity of the continuous place, or by the threshold of the guard arcs connecting it to discrete transitions, as formalized in the following:

$$
\begin{aligned}
& B_{u}\left(P_{i}\right)=\min \left\{\phi_{b}^{\mathcal{P}}\left(P_{i}\right), \min _{T \in \mathcal{G}_{P}\left(P_{i}\right)}\left\{q:(\triangleright, q)=\phi_{u}^{\mathcal{A}}\left(\left\langle T^{d}, P_{i}\right\rangle\right), q \geq x\left(P_{i}\right)\right\}\right\}, \\
& B_{l}\left(P_{i}\right)=\max \left\{\phi_{b}^{\mathcal{P}}\left(P_{i}\right), \max _{T \in \mathcal{G}_{P}\left(P_{i}\right)}\left\{q:(\triangleright, q)=\phi_{u}^{\mathcal{A}}\left(\left\langle T^{d}, P_{i}\right\rangle\right), q \leq x\left(P_{i}\right)\right\}\right\},
\end{aligned}
$$

where $\mathcal{G}_{P}\left(P_{i}\right)$ is the set of connected discrete transitions via guard arcs to $P_{i}$. In words, $B_{u}\left(P_{i}\right)$ is the closest upper boundary that the continuous place $P_{i}$ may reach, if it has positive drift. The same is true for $B_{l}\left(P_{i}\right)$, however, it is the closet lower boundary, which may happen if the place has negative drift.

The procedure FINDNextDtrmEvent, given in Algorithm 5.1, provides the next event for each state of the system. Using the above notations, we iterate over all continuous places in lines 2-8, and determine the closest possible event regarding continuous places. Also for all enabled deterministic timed transitions we have to find the remaining time to fire (lines 9-13). Note the exclusion of general transitions in line 9. Further recall that the function $\Delta\left(T^{d}\right)$ (used in line 11) returns the firing time of the deterministic timed transition $T^{d}$, as defined in Section 3.1, Equation (3.1). Finally, the next event, denoted $e$, is the one with the shortest remaining time to occur, denoted $\Delta t_{e}$.

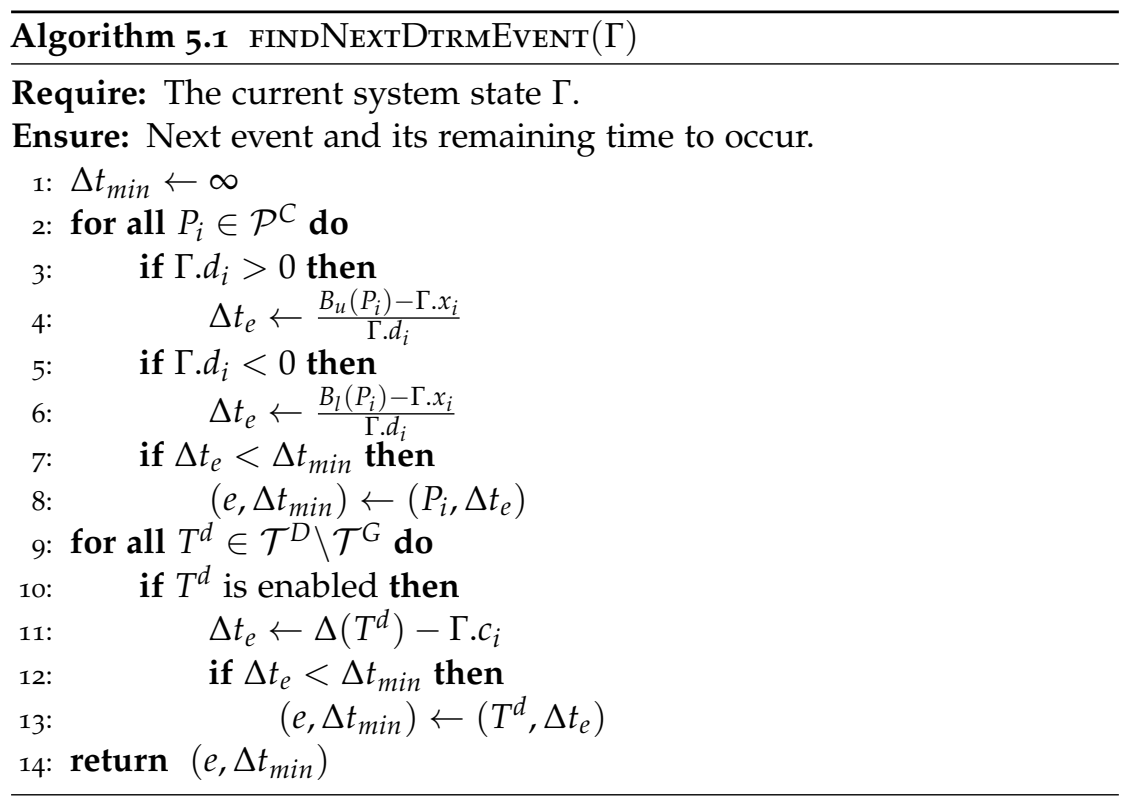

Algorithm 5.2 describes how the area below the line $t=s$ can be partitioned into a set of deterministic regions, denoted by $\mathcal{R}^{D}$. We start at 
time point $t=0$, and continue the process until reaching the maximum time of analysis, denoted by $t_{\max }$. At each time point $t=h_{i}$, using the the the procedure FINDNextDTRMEvent, the next event $e$ along its relative occurrence time $\Delta t_{e}$ is determined. A new region $R_{i}$ is created and added to the set of deterministic regions $\mathcal{R}^{D}$ (lines 6-8, Algorithm 5.2). Then the current system state is updated (Algorithm 5.5, which will be discussed in the next subsection) and the current system time is advanced by $\Delta t_{e}$ time units (lines 9-10).

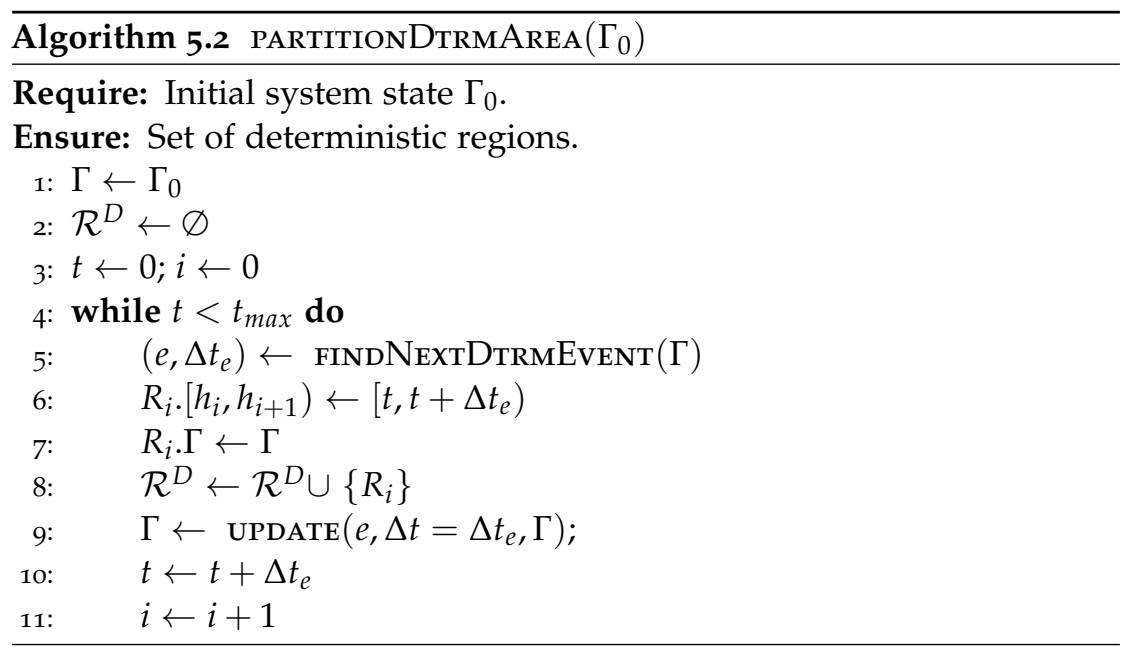

\subsubsection{General transition has fired}

When the general transition has fired we have $t>s$, therefore this phase involves the partitioning the area above the line $t=s$. As shown in Figure 5.1, in the previous phase the line $t=s$ has been split into several line segments, by deterministic regions. We iterate over all these line segments, and if the general transition was enabled in the system state of the corresponding deterministic region, it is fired, and the area above the corresponding line segment needs to be further partitioned. In generation of an STD, line segments describe the occurrence of events, therefore we refer to them as event segments.

Definition 5.1. An event segment $\delta$ is defined as the following structure:

- The equation: $\delta . e q: t=\alpha s+b$

- End points: $\delta .\left[s_{l}, s_{r}\right)$.

Each segment $\delta$ corresponds to an event, and the equation $\delta . e q$ represents its occurrence time as a linear function of $s$. Moreover, the corresponding event occurs at the time determined by the equation $\delta . e q$ if the 
value of the stochastic variable is inside the interval $\delta .\left[s_{l}, s_{r}\right)$. In other words, this event occurs at time $t=\alpha s+b$ if $s_{l} \leq s<s_{r}$. For example if the general transition fires when the system is in the deterministic region $R_{i}$, we enter the area above the segment with equation $t=s$ and endpoints $\left[h_{i}, h_{i+1}\right)$.

Proposition 5.1. After firing the general transition at time s, for each system state, the occurrence time of all next events are linear functions of $s$.

Proof. We prove the proposition by structural induction on associated segments of events, with the segment associated with firing of the general transition as the basis. Without loss of generality, assume that before firing the general transition, the system is in the deterministic region $R_{i}$ (with entry and exit time of $h_{i}$ and $h_{i+1}$, respectively). Consider $P_{k}^{c} \in \mathcal{P}^{C}$ and $T_{k}^{d} \in \mathcal{T}^{D} \backslash \mathcal{T}^{G}$, and let $x_{k}$ and $c_{k}$ be the content of $P_{k}^{c}$, and the value of the clock of $T_{k}^{d}$ at the time of entering $R_{i}$, respectively. When the general transition fires at time $s$, we have been in this region for $\Delta t=s-h_{i}$ time units, so at the firing time of the general transition the fluid level of continues place $P_{k}^{c}$ linearly depends on $s$, as follows $x_{k}^{\prime}=d_{k}\left(s-h_{i}\right)+x_{k} ; h_{i} \leq s \leq h_{i+1}$. Also the clock value of an enabled deterministic timed transition $T_{k}^{d}$ is $c_{k}^{\prime}=\left(s-h_{i}\right)+c_{k} ; h_{i} \leq s \leq h_{i+1}$. Therefore, at the very moment after firing the general transition, all fluid levels and clock values are linear functions of $s$, and hence the occurrence time of the next events are linear function of $s$ too, as shown for the general case below.

As the inductive step, with respect to the induction hypothesis, suppose that an event has occurred at time $t=\alpha s+\beta$ for $s \in\left[s_{l}, s_{r}\right)$. Let the fluid level in a continuous place $P_{k}^{c}$ be $x_{k}=a_{k}^{p} s+b_{k}^{p} ; s_{l} \leq s \leq s_{r}$. The amount of time this place needs to reach one of its boundary is denoted by $\Delta t_{k}^{p}$ and can be calculated as follows:

$$
\Delta t_{k}^{p}= \begin{cases}\left(B_{u}-x_{k}\right) / d_{k} & \text { if } d_{k}>0 \\ \left(B_{l}-x_{k}\right) / d_{k} & \text { if } d_{k}<0\end{cases}
$$

in which $B_{u}$ and $B_{l}$ are upper and lower boundaries for place $P_{k}^{c}$, as defined in Equation (5.1). According to the occurrence of the previous event, we have $\Delta t_{k}^{p}=t_{k}^{p}-(\alpha s+\beta)$ for $s \in\left[s_{l}, s_{r}\right)$, where $t_{k}^{p}$ is the occurrence time of the event corresponding to continuous place $P_{k}^{c}$. As a result, above the line segment $t=\alpha s+\beta ; s \in\left[s_{l}, s_{r}\right)$, the considered event occurs at time $t_{k}^{p}$ which is obtained as follows:

$$
t_{k}^{p}= \begin{cases}\left(\alpha-\frac{a_{k}^{p}}{d_{k}}\right) s+\left(\frac{-b_{k}^{p}+B_{u}}{d_{k}}+\beta\right) & \text { if } d_{k}>0, \\ \left(\alpha-\frac{a_{k}^{p}}{d_{k}}\right) s+\left(\frac{-b_{k}^{p}+B_{l}}{d_{k}}+\beta\right) & \text { if } d_{k}<0 .\end{cases}
$$


The firing time of a deterministic timed transition can be derived in a similar way. Let the clock value of the deterministic timed transition $T_{k}^{d}$ be $c_{k}^{t}=a_{k}^{t} s+b_{k}^{t}$. The firing time can then be calculated as follows:

$$
t_{k}^{t}=\left(\alpha-a_{k}^{t}\right) s+\left(\Delta\left(T_{k}^{d}\right)+\beta-b_{k}^{t}\right)
$$

where $\Delta\left(T_{k}^{d}\right)$ is the firing time of transition $T_{k}^{d}$, as defined in Equation (3.1).

Therefore, the occurrence time of both types of events linearly depend on $s$. Now, following the same argument, we set $\alpha=1$ and $\beta=0$, and the basis of the induction is also satisfied, hence the proof is complete.

As an immediate result of the above proposition we can state that all regions in the area above the line $t=s$ are polygons.

We now proceed to present the algorithm for partitioning the area above an arbitrary underlying segment $\delta$. In order to do this we first need to describe the process for computing the next potential events. The procedure for finding all potential events, at each system state, is called FindPotentialEvents and shown in Algorithm 5.3. It uses the same arguments as in the proof of Proposition 5.1. In lines 2-7 we iterate over all continuous places, and for each with a non-zero drift the time for reaching its boundary is computed according to Equation (5.2). Note that $\Gamma \cdot x_{i}$ is a linear equation in $s$, and $B_{u}\left(P_{i}\right)$ and $B_{l}\left(P_{i}\right)$ are defined in Equation (5.1). Also in lines 8-11 the iteration is performed over all enabled deterministic transitions, and their corresponding firing times are computed according to Equation (5.3). Finally, the set of all events and the equations that specify their occurrence time is returned.

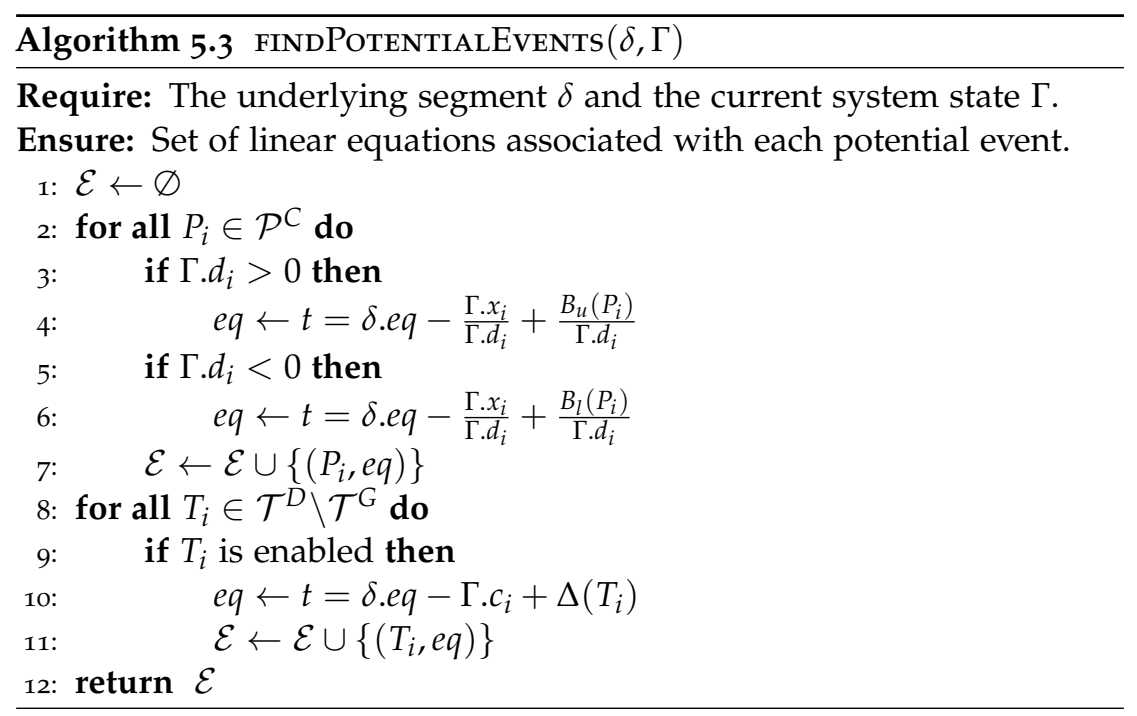


The pseudo-code for partitioning the area above the line $t=s$ is outlined in Algorithm 5.4. First, we check whether the maximum analysis time has not been reached (line 1 ). Then we identify the potential events that can occur in the current marking of the system by calling FINDPOTENTIALEvENTS, which returns the set of linear equations associated with each potential event. In order to find the occurrence times of the next events, we have to take the minimum over all these linear equations, for the values of $s \in \delta .\left[s_{l}, s_{r}\right)$. Taking the minimum over a set of lines results in several convex polygon(s) over the underlying segment $\delta$. Note that these equations are only valid in the area above the underlying segment $\delta$. An example with three possible events: $e_{1}, e_{2}$ and $e_{3}$ is presented in Figure 5.2. Event $e_{2}$ intersects with the underlying segment at point $p$, so $e_{2}$ cannot occur for $s>p$ and in the minimum taking procedure after this point $e_{2}$ does not have to be considered any more. As a result two polygons will be formed over the underlying segment.

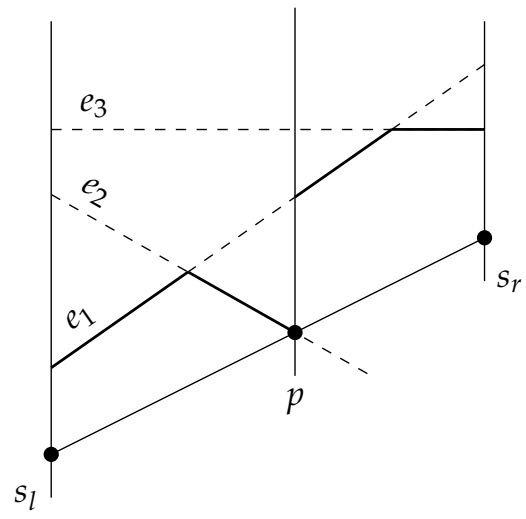

Figure 5.2.: Formation of a region.

The procedure that identifies the set of next events over an underlying segment, $\delta_{0}$, is called FINDNExTEvents, and simply iterates over all lines indicating the firing time of potential events and for all $s \in$ $\delta_{0} \cdot\left[s_{l}, s_{r}\right)$ finds the minimum line. It returns a set of segments from which the polygon(s), i.e., regions, over the underlying segment can be formed by iteration over the set of segments, this is done in procedure CREATEREgIONS. We will formalize these processes for the general case of multiple stochastic variables in the next section.

The procedures FIndPotentialEvents, FINDNextEvents and CREAtePolygons, are called in lines 3-5, in Algorithm 5.4. Now, having obtained the set of segments of all next events, we can partition the area above each of these segments. Through line 6-8 we iterate over all these segments, and recursively call the function PARTITIONABOVESEGMENT for each segment, after updating the system state. 


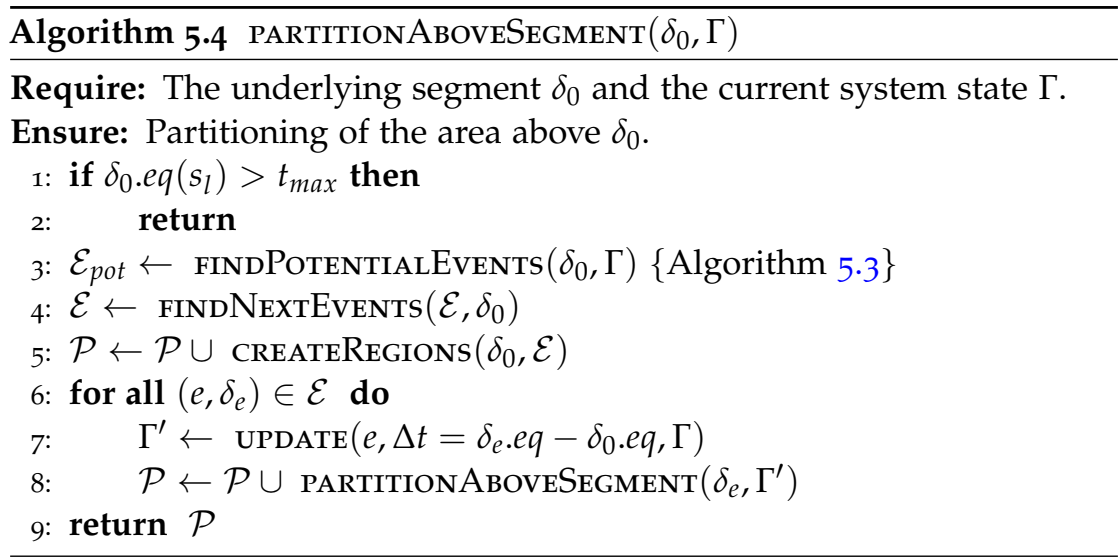

The procedure for updating the system state is provided in Algorithm 5.5. This procedure needs the event $e$ and the amount of time $\Delta t$ that can possibly depend on $s$, to advance the marking. In lines 2-3, for each continuous place $P_{i}$ it alters the fluid level in that place according to $\Delta t$ and the fluid drift $d_{i}$. Also in lines $4-5$, it adds $\Delta t$ to the clock value of each enabled deterministic transition. Moreover, if the event $e$ is a transition it is fired, in line 8 , to update the discrete marking. Finally we update the fluid drifts for the new marking, by calling the procedure AdAptRates, given in Algorithm 3.4, Section 3.5. ${ }^{1}$

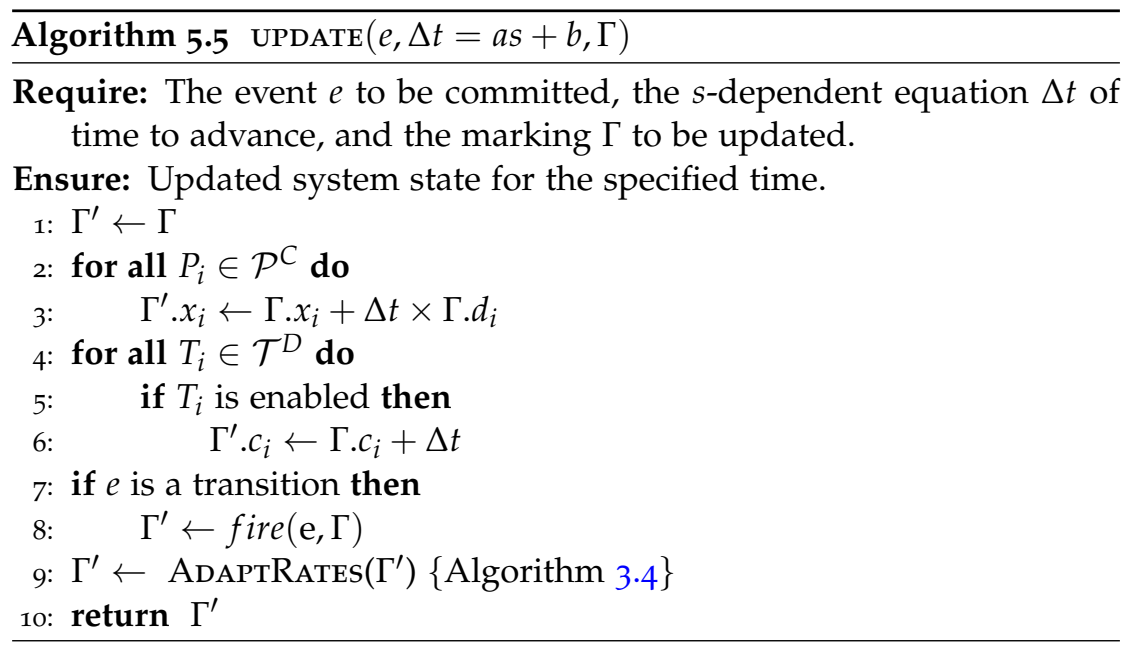

Finally, Algorithm 5.6 generates the STD. First the deterministic regions are stored in the set $\mathcal{R}^{D}$, by calling PARTitionDtrmAreA. Then

1 Note that the signature of the process ADAPTRATEs used here does not match strictly to the one given in Algorithm 3.4. This is because in context of Section 3.5, the structure of HPnG, being analysed, was important, and the marking was assumed to be known implicitly. However, here the vice versa is true. 
for each region $R_{i}$, if the only general transition, denoted by $T^{g}$, is enabled, the segment with equation $t=s$ with the interval $\left[h_{i}, h_{i+1}\right)$ is created (lines 4-6). The marking of the system is updated in line 7 , by calling procedure UPDATE. Since the general transition should be fired we pass $T^{g}$ as argument. Also the time elapsed after entering the deterministic region $R_{i} \in \mathcal{R}^{D}$ before firing the general transition, is $\Delta t=s-h_{i}$; this is passed as the second argument. Finally the procedure PARTITIONABOvESEGMENT is called with two arguments the created segment $\delta$ and the updated system state $\Gamma^{\prime}$.

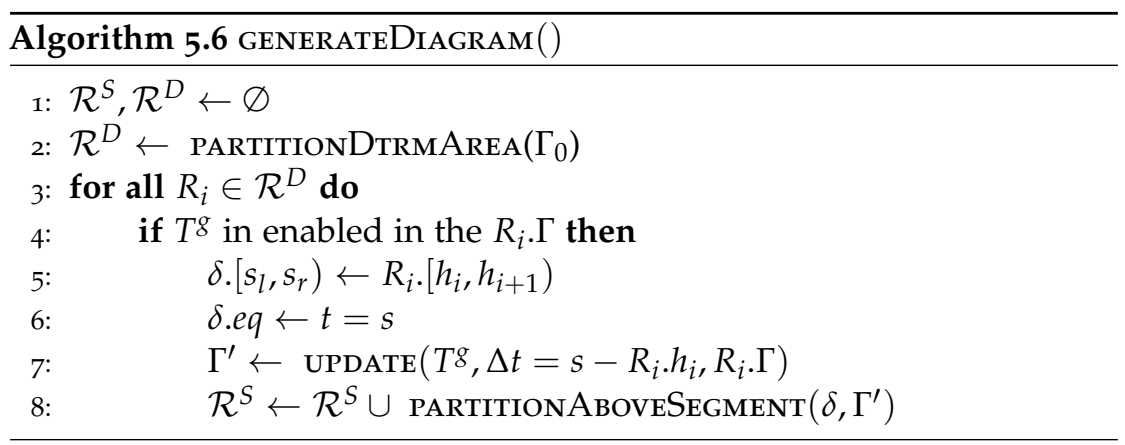

\subsection{MULTIPLE GENERAL TRANSITIONS}

In this section we generalize the idea of generation of STD for one stochastic variable to the case of multiple stochastic variables. As mentioned earlier in Section 4.1 adding an extra stochastic variable will increase the dimension of STD by one, and hence will add to the complication of the computational geometry algorithms for generation of STD. More specifically, instead of dealing with line segments and polygons in two dimensions as in Section 5.1, now we have to deal with hyperplanes and polytopes, respectively.

\subsubsection{Preliminaries}

We show the above claim holds for higher dimensions by proving the analogous version of the Proposition 5.1. More formally, we show that the value of a continuous variable ${ }^{2}$ is a linear function of vector of value of stochastic variables, and the current time of evolution. In other words, let $n$ be the arbitrary number of stochastic variables present in the system $^{3}$, and $\mathbf{s}=\left(s_{1}, \ldots, s_{n}\right)$ be a value vector for stochastic variables, and

2 Recall that a continuous variable is either clock of a discrete transition, or content of a continuous place

3 Recall from Section 4.1.2 that we know the number of stochastic variables prior to the generation of STDs. 
let $x(\mathbf{s}, t)$ be an abstract realization of a continuous variable, then we have:

$$
x(t, \mathbf{s})=a_{n+1} t+\sum_{k=1}^{n} a_{k} s_{k}+a_{0}=a_{n+1} t+\mathbf{a . s}+a_{0} .
$$

Where $a_{i} \in \mathbb{R}^{\prime}$ s, for $0 \leq i \leq n+1$ are constant coefficients, and $\mathbf{a}=$ $\left(a_{1}, \ldots, a_{n}\right)$.

Proposition 5.2. At each time point $t$ during the evolution of the system, the value of the continuous variables and the occurrence time of the next events can be characterised as a linear equation of $t$ and vector $\mathbf{s}$.

Proof. We use induction on the number of events. Assume the previous event has occurred at time $t_{0}=\alpha . \mathbf{s}+\alpha_{0}$, in which $\alpha$ is a vector of $n$ scalars. If no general transition has fired yet, $t_{0}$ is a constant. Moreover, $t_{0}=s_{k}$ corresponds to the firing time of the $k$-th general transition. We calculate the occurrence time of the next event due to a continuous place reaching its upper boundary (other event types can be seen as simpler version of this type). For this case, the place with $x$ amount of fluid, which changes with drift $d>0$, will reach its upper boundary $B$, according to the following equation:

$$
B=x\left(\mathbf{s}, t_{0}\right)+d \Delta t_{e}=x\left(\mathbf{s}, t_{0}\right)+d\left(t_{e}-t_{0}\right),
$$

in which $\Delta t_{e}=t_{e}-t_{0}$ is the relative occurrence time of this event with respect to the previous event occurred at $t_{0}$, and $t_{e}$ is the absolute occurrence time of the event. Hence, we have:

$$
t_{e}=-(1 / d) x\left(\mathbf{s}, t_{0}\right)+t_{0}+B / d,
$$

which is a linear equation of $\mathbf{s}$. Now each continuous variable can be updated based on $\Delta t_{e}$ and their constant drift, which again results in a linear equation.

\subsubsection{Facets and regions}

As a result of the Proposition 5.2, like the case of single stochastic variable, occurrence of events which characterize the boundaries between regions are specified by linear functions of $\mathbf{s}$ and $t$. This means in a STD of $n$ stochastic variables, events and regions are represented by hyperplanes and polytopes of $n+1$ dimensions, respectively.

In order to partition the state space, after the occurrence of each event, we have to find the equations for all potential next events, and take the minimum over them. Geometrically, this corresponds to finding the lower envelope of a set of hyperplanes (visualized in Figure 5.5), which results in a set of facets. A facet is a confined version of a (hyper)plane, 
i.e., it is limited by its set of borders. Facets in higher dimensions, correspond to segments in two dimensions. Whereas a segment is represented by a line and an interval, a facet is characterized by a hyperplane and a set of boundaries. Each event is associated with an event facet as shown in Figure 5.3 for a model with two stochastic variables.

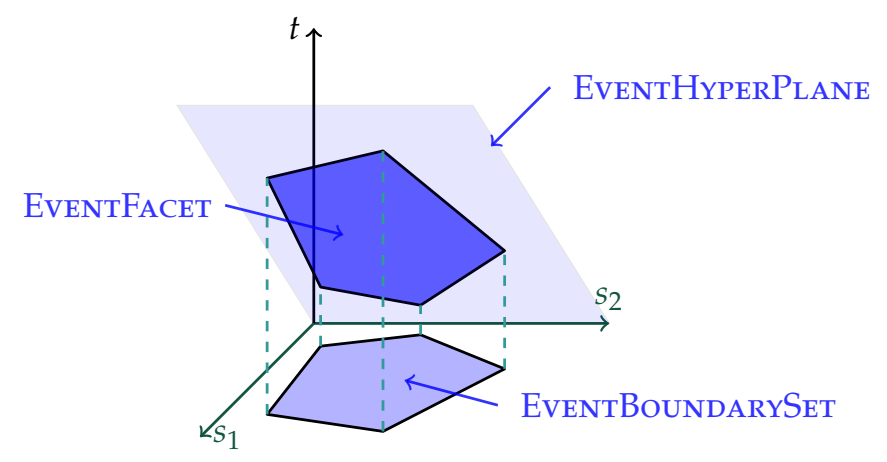

Figure 5.3.: Demonstration of an event facet, in 3 dimensions.

Definition 5.2. An event facet is defined as the following structure:

EvENTFACET:

EVENTHYPERPLANE: $t=\sum_{k=1}^{n} a_{k}^{\prime} s_{k}+a_{0}^{\prime}$

EvEntBoundarySET: $\left\{\sum_{k=0}^{n} b_{k} s_{k}+b_{0} \leq 0\right\}$

The EventHyperPlane shows the time $t$ at which the event corresponding to this facet is happening. The EventBoundarySET defines the volume containing values of vector $\mathbf{s}$. This is analogous to the intervals in case of having one stochastic variable. This means the corresponding event to the facet will happen at time $t$ given by equation in EventHyperPlane, while $\mathbf{s}$ is in the volume given in EventBoundARYSET. These concepts are visualized in Figure 5.3 for the case of two stochastic variables.

A region as defined in Definition 4.1, is now the maximal volume surrounded by a set of neighbouring event facets. Similar to the case of one stochastic variable, for all points in a region the possible values of a continuous variable can be represented by a linear equation of time and values of stochastic variables, as given in Equation (5.4).

\subsubsection{Partitioning and Generation}

The algorithm for partitioning the state space above a given facet $\mathcal{F}$, with prior marking $\Gamma$ is given in Algorithm 5.7. The function computeNexTEvents is the analogous version of FIndPotentialEvents (Algorithm 5.3) for higher dimension. It solves a set of linear equations, as suggested 
in Proposition 5.2, and returns a set of hyperplanes, where each of its elements corresponds to the occurrence time of a potential next event. Since we are interested in finding the next occurring events, which depend on $\mathbf{s}$, we have to find the minimum over all occurrence times of these potential events. The function CREATEREGIONs, finds this minimum over the given hyperplane set, which is returned by the function COMPUteNextEvents, and then creates the set of regions formed above the given event facet (we will provide a detailed description of this function below).

Subsequently, in lines 4-8, Algorithm 5.7 iterates over the sets of facets forming each returned region. If the new event facet does not correspond to reaching the maximum time of analysis, $t_{\max }$ (line 6 ), the algorithm updates the system state $\Gamma$, by calling the function UPDATE (given in Algorithm 5.5), which updates the values of all the continuous variables, based on the time difference of $\mathcal{F}$ and the new event facet $f_{j}\left(f_{j}\right.$.e denotes the event associated with facet $f_{j}$ ). Note that, if the new event facet corresponds to the firing of a general transition, i.e., $f_{j} . e \in \mathcal{T}^{G}$, the vector $\mathbf{g}$ defined in Section 3.2.3, needs to be updated (this is taken care of in line 8 of Algorithm 5.5). Finally, the algorithm recursively calls itself over each new facet with the updated system state.

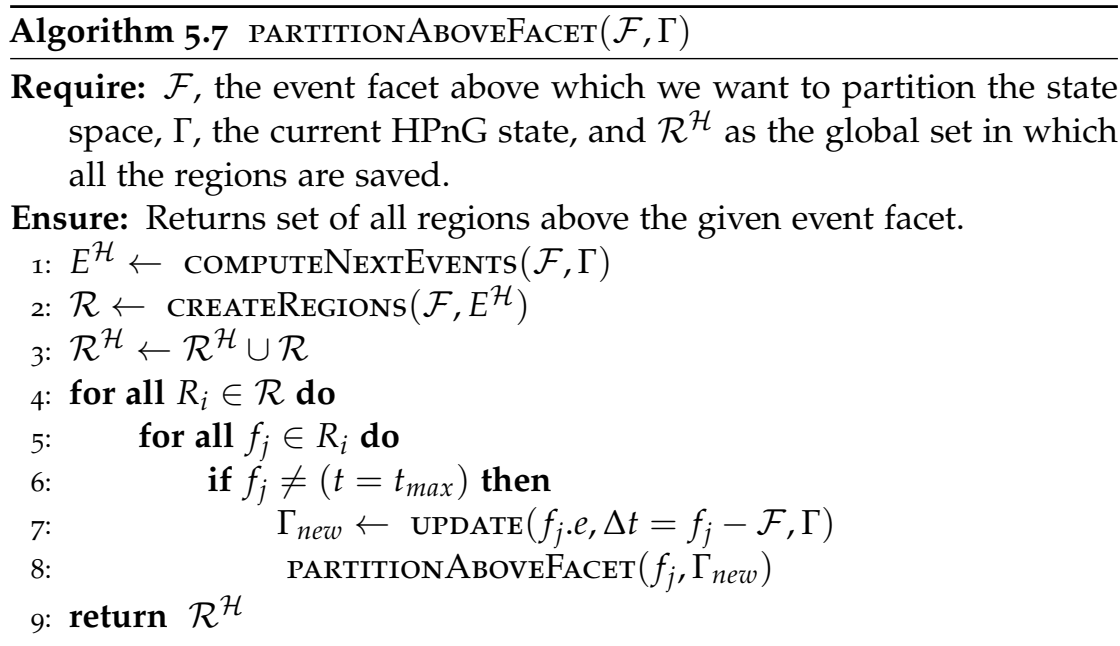

Define $\mathcal{F}_{0}$ as the initial event facet, with EventHyperPlane : $t=0$, and EventBoundarySet : $\left\{0 \leq s_{i} \leq t_{\max }\right\}_{i=0}^{n}$, and let $\Gamma_{0}$ be the initial marking of the HPnG. Now if we call the function PARTITIONAвOvEFACET given in Algorithm 5.7, with $\mathcal{F}_{0}$ and $\Gamma_{0}$ as arguments, the entire state space is partitioned, i.e., the STD is generated.

The function CREATEREgIONS embodies the implementation of the main challenge for handling multiple general transitions, which is presented in Algorithm 5.8. Since each hyperplanes associated with event 
may intersect with the underlying event facet, there is the possibility of having a set of regions, like the analogous 2-dimensional case shown in Figure 5.2. For the case of two stochastic variables (3 dimension), this possibility is depicted in Figure 5.4. Note that, the intersection of a hyperplane with a facet in 3 dimensions is a line. As illustrated in the figure, the facet intersects three hyperplanes, and as a result forms six sub-facets. Above each of these sub-facets we have to form a region. The problem of forming these sub-facets in 2 dimensions is known as arrangement of lines, and in higher dimension as arrangement of hyperplanes [65]. This is an essential problem in computational geometry, since many other problems can be reduced to it [66].

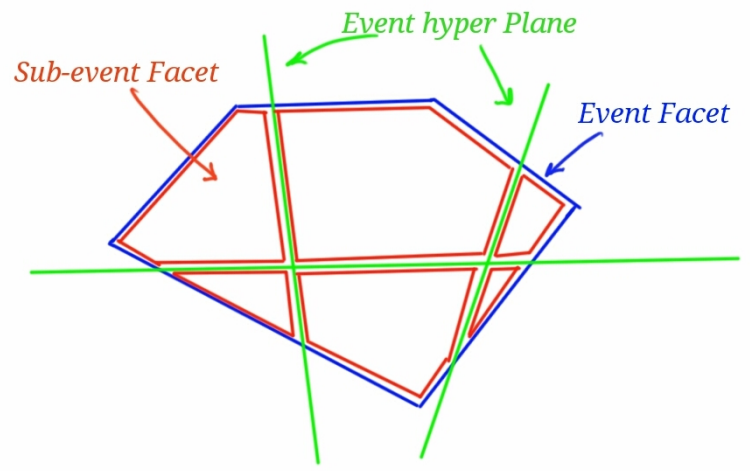

Figure 5.4.: Top view of possible intersection of an event hyperplane with event facets, and formation of sub-facets.

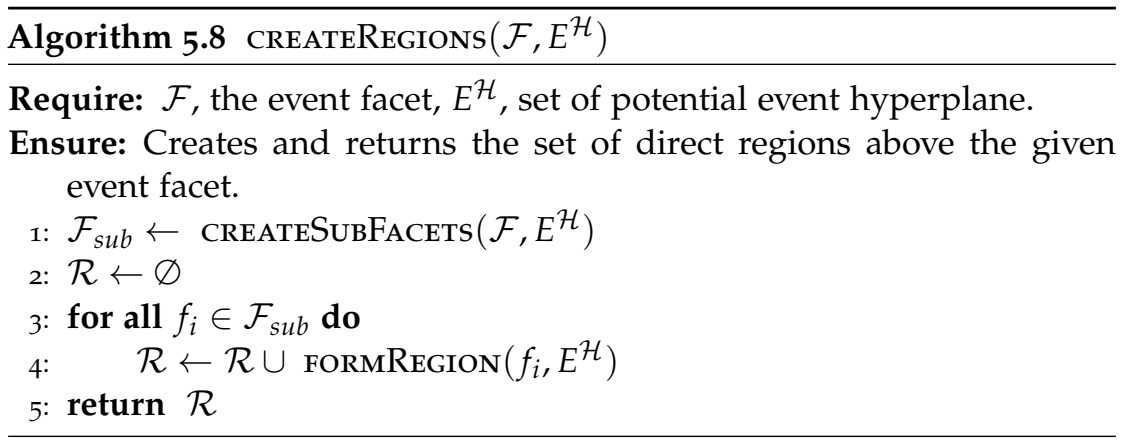

After having determined all sub-facets, we have to form the regions above each of them. This is done by taking the minimum over all the potential event hyperplanes. This problem can be interpreted as the intersection of a set of half spaces. More formally, let $\mathcal{F}$ be a sub-facet with hyperplane $t=\mathbf{a} . \mathbf{s}+a_{0}$, and the set of $m$ boundaries $\left\{\mathbf{b}^{i} . \mathbf{s}+b_{0}^{i}=\right.$ $0\}_{i=1}^{m}$. Moreover, let $\left\{t=\mathbf{c}^{j} . \mathbf{s}+c_{0}^{j}\right\}_{j=1}^{l}$ be the set of $l$ potential event 
hyperplanes. Then the region formed above the event facet $\mathcal{F}$ is the intersection of the following half spaces:

$$
\begin{cases}t-\mathbf{a . s}+a_{0}>0 & \\ \mathbf{b}^{i} . \mathbf{s}+b_{0}^{i} \leq 0, & 1 \leq i \leq m \\ t-\mathbf{c}^{j} . \mathbf{s}+c_{0}^{j}<0, & 1 \leq j \leq l .\end{cases}
$$

This can be determined while creating the sub-facets in the previous phase. The formation of a region is visualised in Figure 5.5. As can be seen, a region (the transparent volume), is formed as the interior space of an event sub-facet, sub-facet boundaries, and potential event hyperplanes. Informally, these can be interpreted as, floor, columns, and roofs, respectively.

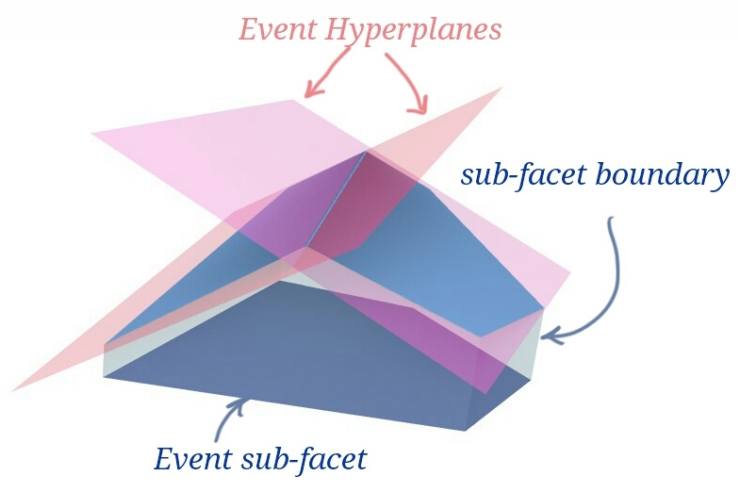

Figure 5.5.: Formation of a region over an event facet.

\subsubsection{Finiteness and termination}

Even though generation of STD is always performed for a given and finite time bound, $t_{\max }$, there is still the possibility of having an infinite number of regions in the STD before a finite time bound. This is known as Zeno behaviour as discussed in Section 2.2.2. This happens whenever an infinite sequence of vanishing markings occurs (Definition 2.5). This problem is well-known for all Petri net formalisms that allow immediate transitions. However, if we require that models to be bounded, as defined in Definition 2.3, infinite sequences of vanishing markings can only take place in the form of cycles of vanishing markings, which can be detected and removed [67-69]. This ensures that we can always reach a tangible marking in a finite number of steps. Therefore we can make sure that the number of regions in the STD before a finite time bound is also finite. Hence, for a bounded model, a finite number of general 
transition firings, and a finite time bound our algorithm for generation of STD will always terminate.

\subsubsection{Complexity}

The presented algorithm for generation of STD approaches the problem for the general case of $n$ stochastic variables. However, as mentioned earlier, the introduced algorithm depends on two well-known computational geometry problems, namely half-space intersection and hyperplane arrangement. The function FORMREGION embodies the former problem, and the latter is presented in the function CREATESubFACETS. The problem of half-space intersection, is dual to the convex-hull problem, which can be solved in order $O(m \log m)$, where $m$ is the number of half-spaces [64].

However, the complexity of the existing algorithm for hyperplane arrangement, is exponential in dimension $d$, i.e., $O\left(m^{d}\right)$, where $m$ is the number of hyperplanes [70]. In our case, $d$ is equal to the number of stochastic transition firings plus an extra dimension for time. To the best of our knowledge, there is no implementation of hyperplane arrangement for more than two dimensions. Hence, we are currently restricted to the case, where only two stochastic variables are available in the system. For both of the above problems for case of $d \leq 3$, there are robust implementations and libraries, among which we have used Computational Geometry Algorithm Library (CGAL), which provides extensive implementations for most of the existing algorithms for solving halfspace intersection and hyperplane arrangement [71-73].

\subsubsection{Reservoir Example: revisited}

Figure 5.6, shows an extended version of the reservoir example given in Section 4.1.1, with two general transitions. The continuous place $P_{r}$, models a water reservoir with capacity 10. This place is being filled using two different producer pumps, modelled as continuous transitions $T_{1}$ and $T_{2}$, with nominal rates 1 and 2 , respectively. The water in the reservoir is being consumed by the demand pump $T_{d}$, at rate 2 . We know that after 5 hours, the demand will stop, i.e., the deterministic transition $T_{S}$ will fire at $t=5$. Two general transitions $G_{1}$ and $G_{2}$, are modelling the possibility of failure in the production. They fire according to predefined probability distributions (not provided here, because we are now only concerned with generation of STD).

Figure 5.7, shows the 3-dimensional Stochastic Time Diagram for this example, with the initial empty reservoir. Each region is depicted with a different color. Figure 5.7a, illustrates the phase in which no general transition has fired yet. Note that that this is similar to the 2-dimensional 


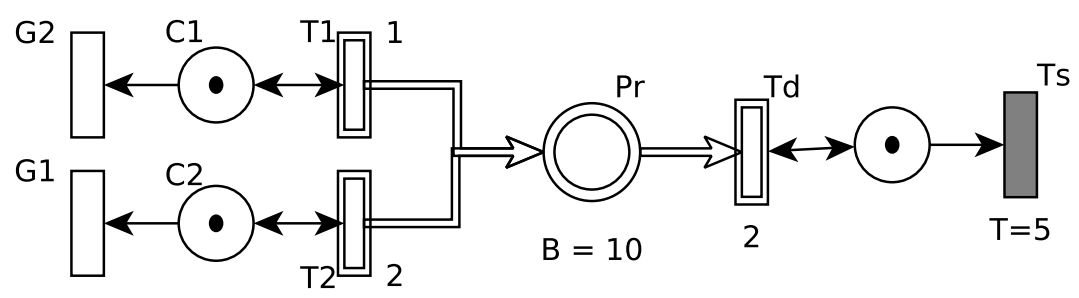

Figure 5.6.: A reservoir model with two different production rates.

Figure 5.1. The boundary between red and green regions, corresponds to the firing of deterministic transition $T_{s}$, i.e., the plane $t=5$. After firing of $T_{S}$ the reservoir contains 5 units of water, and is being filled with rate 3 . Hence it reaches its upper boundary at time $t=5+5 / 3$, which is the plane representing the boundary between green and purple regions. After this nothing happens until reaching $t_{\max }$ As can be seen the formation of these regions, while the the general transitions has not fired, is independent of the value of $s_{1}$ and $s_{2}$, i.e., they can be characterized by planes parallel to $\left(s_{1}, s_{2}\right)$-plane. Moreover, the planes $t=s_{1}$ and $t=s_{2}$, which correspond to the firing of general transitions, are clearly visible.

Figures $5.7 \mathrm{~b}-5.7 \mathrm{e}$, represent later phases in generation of STD. For instance Figure $5.7 b$, is the formation of regions after firing of $G_{2}$. In this case the only possible event to happen is the firing of $G_{1}$, and after that reaching the maximum time $t_{\max }$. Therefore only two regions are formed. Finally, Figure 5.7c, depicts the complete STD of the extended reservoir example. From these pictures it is apparent how the shape of regions above the two planes $t=s_{1}$ and $t=s_{2}$ depends on the values of $s_{1}$ and $s_{2}$.

\section{$5 \cdot 3$ RELATED WORK}

There are many systems in the real world which encompass both stochastic and hybrid characteristics, therefore from a practical point of view it is crucial to integrate stochasticity into hybrid models. Because of this, there is an extensive literature on formalization and development of the so-called Stochastic Hybrid Models (SHMs). There are different approaches to integrating stochastic behaviours into systems with hybrid characteristics., and the difference between these approaches mainly lies on how the the stochasticity is included in the system.

Generally speaking, all hybrid systems consist of different modes in each of which each continuous variable is associated with a specified rule, governing its evolution, e.g, a differential equation. Moreover, it 


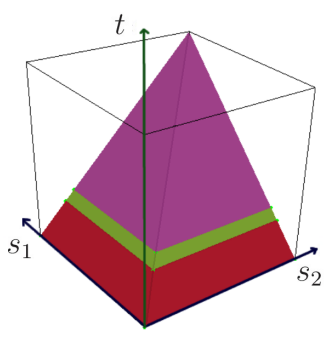

(a) No g-trans. fired.

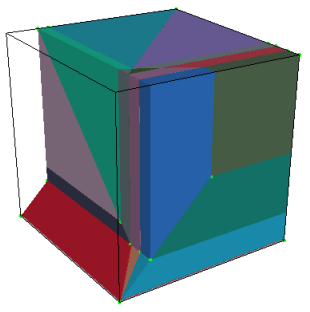

(d) A middle phase.

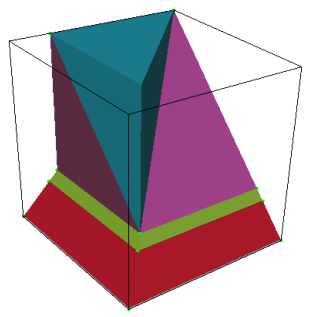

(b) A middle phase.

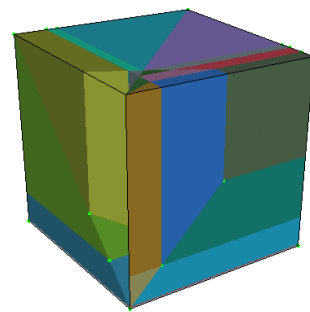

(e) A middle phase.

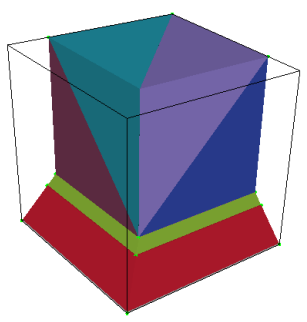

(c) A middle phase.

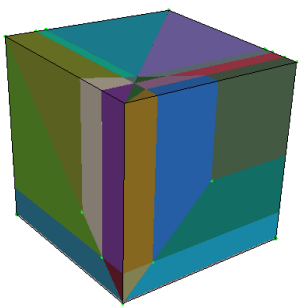

(f) Complete STD

Figure 5.7:: STD for the reservoir example.

is specified how to move between each of these modes. Now, roughly speaking, in order to integrate stochasticity into these hybrid models one can take two approaches. One approach is to replace the differential equations describing the evolution of continuous variables in each mode of hybrid models with stochastic differential equations, such as Switched Diffusion Processes [9], and the so-called Stochastic Hybrid Systems [10]. These approaches are usually theoretical and development of computational tools and numerical methods for them is crucial. These include, for instance, approximating the infinite state problem with a finite one [74], or performing simulation methods [75]. There are also methods for carrying out reachability analysis using temporal logic, based on idea of converting stochastic hybrid systems to finite state Markov chains, which is later analyzed using model checkers [76].

Another approach for integrating randomness in hybrid systems, is to replace transitions between modes of hybrid systems with probabilistic jumps, examples include Piecewise-deterministic Markov processes [8], Probabilistic Hybrid Automata [14], and Fluid Stochastic Petri nets [16].

For hybrid Petri nets, modes of system is defined as the state of the system in which evolution of content of continuous places and clock values, which include all the continuous variables, are determined by simple differential equations (cf. Corollary 3.1). Moreover, firings of discrete transitions cause the system to make a transition among these 
modes. One should be able to tell that, in HPnGs we have undertaken the second approach, because the stochasticity is included in the system by firings of general transitions.

The idea of the conditioning the state space of Petri nets with generally distributed transitions, is first introduced in [26]. The method is called Parametric Reachability Analysis, and is done by computing all reachable locations, by separating the deterministic and the stochastic evolution of the system. The main contribution of this work is to provide the practical possibility of handling arbitrary number of continuous places. The concept of parametric reachability analysis resembles the state-classes presented in [54], which excludes continuous variables.

Parametric Reachability Analysis suffers in two different aspects. Firstly, it is limited to the case in which only one general one-shot transition can be present in the system, i.e., only one stochastic variable is allowed into the analysis. Secondly, at the end computing measures of interests is based on discretization of support of stochastic variables, which results in loosing exactness. Moreover, for each discretized value of stochastic variable one traverse of state space is needed, which makes it costly to get a near exact result for a complex system. The method of generating Stochastic Time Diagrams is aimed to overcome these two mentioned limitations. In the next chapter (Section 6.3) after introducing the model checking algorithms, we compare the performance of the parametric reachability method with the method introduced in this thesis. 



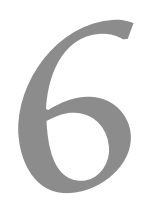

MEASURE COMPUTATION AND MODEL CHECKING

The content of this chapter is based on:

[51] H. Ghasemieh, A. Remke, B.R. Haverkort, and M Gribaudo. Region-based analysis of hybrid Petri nets with a single general one-shot transition. In Formal Modeling and Analysis of Timed Systems, volume 7595 of LNCS, pages 139-154. Springer, 2012

[52] H. Ghasemieh, A. Remke, and B. R. Haverkort. Survivability evaluation of fluid critical infrastructures using hybrid Petri nets. In 19th Pacific Rim International Symposium on Dependable Computing, pages 152-161. IEEE, 2013

[63] H. Ghasemieh, A. Remke, and B. R. Haverkort. Hybrid Petri nets with multiple stochastic transition firings. In 8th International Conference on Performance Evaluation Methodologies and Tools, pages 217-224. ICST, 2014.

In this chapter we investigate how one can compute some measures of interest related to system properties, using the generated STD. We use Stochastic Time Logic (STL) as introduced in Section 4.2, to specify properties of interest. We follow the same scheme as in the previous chapters, by first discussing the concepts for the case of one active stochastic variable and then extending it to the case of multiple stochastic variables.

We consider two types of properties separately, the properties with, and without the Until operator. As given in definition of STL (Definition 4.3) we call the former state-based formulas, and the latter Until formulas. This distinction is considered because investigating properties with Until operator is more involved, hence, needs different algorithms.

At the end of each section in this chapter, whenever we have introduced a method for computing a measure, we will also provide a detailed example in order to show the feasibility of the approach. We reserve real world applications for Chapter 8 and Appendix A.

This chapter is further organized as follows: Section 6.1 investigates how the probability for a state-based formula to hold can be computed. First, the case of one stochastic variable is discussed, and later it is for- 
malized for the case of multiple stochastic variables. Moreover, two detailed examples are provided to show the feasibility and scalability of computations. Section 6.2 introduces algorithms for model checking Until formulas. At the end of this section also a detailed example with interpretation of the results of algorithms is provided. Finally, in Section 6.3, we review the related work on model-checking (stochastic) hybrid systems.

\subsection{MODEL CHECKING STATE-BASED FORMULAS}

In this section we confine ourselves to STL formulas without the Until operator, and later in the next section we will consider Until formula separately. Therefore, in essence, only conjunction and negation of atomic formulas are discussed in this section:

$$
\Psi:=\mathrm{tt}\left|x_{P} \geq c\right| m_{P}=a|\neg \Psi| \Psi \wedge \Psi \text {. }
$$

We start in Section 6.1.1 with the case of one active stochastic variable, and consider only atomic properties. Later, in Section 6.1.2 we extend and formalize the state-based model checking for the case of multiple stochastic variables.

\subsubsection{One stochastic variable: atomic properties}

Having generated the STD, the state of the system depends on the distribution of the firing time of the general transition, and on the system time. By deconditioning $s$, the firing time of the general transition, over the values of it distribution, $g(s)$, the state probability distribution at a given time $\tau$ can be derived, as briefly sketched at the end of Section 4.1.1. To compute the probability of being in a system state for which property $\Psi$ holds at time $\tau$, we first have to identify all regions the system can be in, i.e., all regions intersecting line $t=\tau$. Then we verify whether property $\Psi$ holds for any of these regions, and if so, determine the intervals in which the property is satisfied. Finally, $g(s)$ is integrated over all these intervals.

Based on the definition of regions given in Definition 4.1, an atomic discrete property $\left(m_{p}=a\right)$, either holds in the entire area of a region or not at all. Recall, that the content of a continuous place depends linearly on $s$. Hence, an atomic continuous property $\left(x_{k} \leq b\right)$, may be valid only in a certain part of the considered region. More specifically, let the content of a continuous place $P_{k}$ be $x_{k}=\alpha s+\beta$. For the computation of the probability to be in a system state for which $x_{k} \leq b$ holds, $s^{*}=(b-\beta) / \alpha$ defines the threshold value of $s$ where the validity of the property changes. In case the point $\left(\tau, s^{*}\right)$ lies inside the considered 
region, depending on the sign of $\alpha$ the property is satisfied either before or after $s^{*}$.

Figure 6.1, illustrates the above explanation. An arbitrary region, $R$ is intersected with line $t=\tau$, and the interval in which the line $t=\tau$ intersect the region $R$ is denoted by $\left(s_{l}, s_{r}\right)$. The line representing the content $x_{k}$ of place $P_{k}$, is shown in blue color, and also $s^{*}$ which is the result of solving the inequality $x_{k} \leq b$ is shown. Finally, the validity interval of for the continuous atomic property $x_{k} \leq b$, is illustrated, for bath cases $\alpha>0$ and $\alpha<0$.

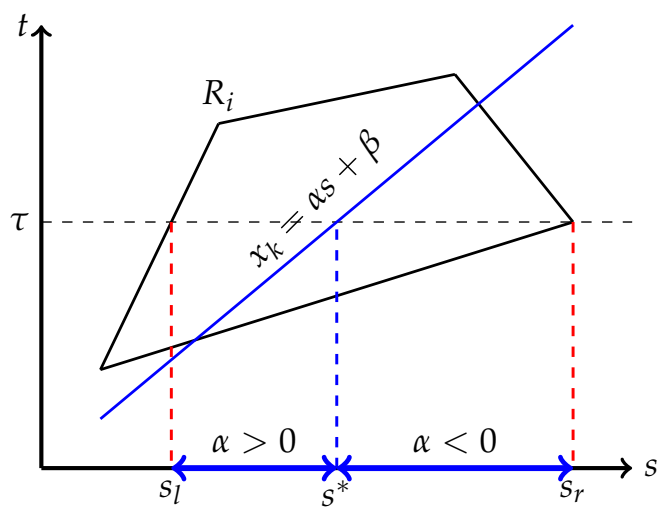

Figure 6.1.: Validity interval for a continuous atomic property $x_{k} \leq b$.

Now let $\mathcal{R}_{\tau}^{S}$ be the set of all regions above line $t=s$, intersecting the line $t=\tau$. For a given region $R_{i} \in \mathcal{R}_{\tau}^{S}$, the set of intervals in which the property $\Psi$ holds is indicated by $\mathcal{I}_{\Psi}\left(R_{i}\right)$ and each interval in this set is denoted $\left[s_{l}^{i}, s_{r}^{i}\right)$. Trivially, $\mathcal{I}_{\Psi}^{i}$ is empty if property $\Psi$ is not satisfied in $R_{i}$. Moreover, Let $R_{\tau}^{D}$ denote the deterministic region, i.e., the region under the line $t=s$ for which $\tau \in\left[h_{1}^{\tau}, h_{2}^{\tau}\right)$, where $h_{1}^{\tau}$ and $h_{2}^{\tau}$ are the enter and exit time to the region $R_{\tau}^{D}$, as introduced in Section 5.1. The probability distribution to be in a system state for which property $\Psi$ is satisfied at time $\tau$ can now be computed as follows:

$$
\begin{aligned}
\operatorname{Prob}^{\tau}(\Psi) & =\int_{0}^{\infty} \mathbb{1}_{\Psi}(\Gamma(s, \tau)) d G(s) \\
& =\int_{0}^{\tau} \mathbb{1}_{\Psi}(\Gamma(s, \tau)) d G(s)+\int_{\tau}^{\infty} \mathbb{1}_{\Psi}(\Gamma(s, \tau)) d G(s) \\
& =\sum_{R_{i} \in \mathcal{R}_{\tau}^{S}} \int_{\mathcal{I}_{\Psi}} d G(s)+\mathbb{1}_{\Psi}\left(R_{\tau}^{D}\right) \int_{\tau}^{\infty} d G(s) \\
& =\left(\sum_{R_{i} \in \mathcal{R}_{\tau}^{S}\left[s_{l}^{i}, s_{r}^{i}\right) \in \mathcal{I}_{\Psi}\left(R_{i}\right)}\left(G\left(s_{r}^{i}\right)-G\left(s_{l}^{i}\right)\right)\right)+\mathbb{1}_{\Psi}\left(R_{\tau}^{D}\right)(1-G(\tau))
\end{aligned}
$$


The above set of equations shows how the partitioning into regions can be used for smarter deconditioning. The last line in Equation (6.1) consists of two terms. The first term expresses the probability of holding $\Psi$ at time $\tau$, when the general transition has fired. This term is computed by simply iterating over all regions above the line $t=s$, intersecting the line $t=\tau$ and accumulating the probability over all intervals in which the property holds. The second term expresses the probability of being in a deterministic region $R_{\tau}^{D}$ in which the property $\Psi$ holds. Note that in a deterministic region since there is no dependency on $s$, either property $\Psi$ holds in the entire region, or does not hold at all. Therefore, $\mathbb{1}_{\Psi}\left(R_{\tau}^{D}\right)$ is 1 if $\Psi$ holds in $R_{\tau}^{D}$ and 0 otherwise.

\subsubsection{Multiple stochastic variable: model checking state-based formula}

In this section we consider the case that $n$ stochastic variables are present in the system, and formalize the general case for model checking statebased formulas. The process is almost the same as in the previous section, except that by moving to higher dimensions, regions become polytopes and their intersections have to be determined by the horizontal hyperplane $t=\tau$. Then we project the intersection results over the s-plane and integrate all CDFs $G_{i}\left(s_{i}\right)$ over the resulting area. Note that here we again assume that random variables corresponding to the stochastic variables are all independent, hence, we can use the product form for the joint distribution.

Like before, an atomic discrete property $\left(m_{p}=a\right)$, either holds in the entire region or not at all. The validity of an atomic continuous property $\left(x_{k} \leq b\right)$, depends on the value of the vector s. More specifically, at a given time $\tau$, the value of continuous variable $x$ is a linear function of vector $\mathbf{s}$. Therefore, the validity space of an atomic continuous property is the half space $x(\tau, \mathbf{s}) \geq b$, i.e., all the $(\tau, \mathbf{s})$ points inside the considered region for which their associated system state $\Gamma(\mathbf{s}, \tau)$, satisfies the given property.

More formally, let $\mathcal{R}_{\tau}$ be the set of regions intersecting with hyperplane $t=\tau$, and for $R_{i} \in \mathcal{R}_{\tau}$, let $A^{\tau}\left(R_{i}\right)=\left\{\mathbf{s}:(\tau, \mathbf{s}) \in R_{i}\right\}$ be the area of s-points which lies in the intersection of $t=\tau$ and $R_{i}$. Then the area of s-points satisfying discrete and continuous atomic properties at time $\tau$ in $R_{i}$ are defined as:

$$
\begin{aligned}
& A_{m_{P}=a}^{\tau}\left(R_{i}\right)= \begin{cases}A^{\tau}\left(R_{i}\right) & \text { if } \exists(t, \mathbf{s}): \Gamma(t, \mathbf{s}) \models\left(m_{p}=a\right), \\
\varnothing & \text { otherwise. }\end{cases} \\
& A_{x_{P} \geq b}^{\tau}\left(R_{i}\right)=\left\{\mathbf{s}: \mathbf{s} \in A^{\tau}\left(R_{i}\right) \wedge x(\tau, \mathbf{s}) \geq b\right\} .
\end{aligned}
$$

Note that, since regions are polytopes, $A^{\tau}\left(R_{i}\right)$ also characterizes a polytope (with one less dimension) in the s-plane, for instance a polygon in the case of two stochastic variables. Because of the linear nature of in- 
equality $x(\tau, \mathbf{s}) \geq b, A_{\Psi}^{\tau}\left(R_{i}\right)$ also defines a polytope. Now conjunction and negation of atomic properties correspond to boolean set operations on these polytopes. More specifically, we have:

$$
\begin{aligned}
A_{\neg \Psi}^{\tau}\left(R_{i}\right) & =A^{\tau}\left(R_{i}\right) \backslash A_{\Psi}^{\tau}\left(R_{i}\right), \\
A_{\Psi_{1} \wedge \Psi_{2}}^{\tau}\left(R_{i}\right) & =A_{\Psi_{1}}^{\tau}\left(R_{i}\right) \cap A_{\Psi_{2}}^{\tau}\left(R_{i}\right) .
\end{aligned}
$$

When two or three stochastic variables are present, the problem of finding validity area of conjunction and negation of atomitc properties in region, reduces to boolean operations on polygons or $3 \mathrm{D}$ polyhedrons, for which efficient implementations exist $[77,78]$.

Finally, the validity area of a certain property at a given time $\tau$ is defined as:

$$
A_{\Psi}^{\tau}=\bigcup_{R_{i} \in \mathcal{R}_{\tau}} A_{\Psi}^{\tau}\left(R_{i}\right) .
$$

Having defined the validity areas, we need to integrate the distribution functions over the validity area in order to find the probability of the given property:

$$
\operatorname{Prob}^{\tau}(\Psi)=\int_{A_{\Psi}^{\tau}} d G(\mathbf{s}),
$$

where $G(\mathbf{s})$ is the joint probability distribution for which, because of the independence assumption, we have: $G(\mathbf{s})=\prod_{k=1}^{n} G_{k}\left(s_{k}\right)$.

As mentioned earlier, $A_{\Psi}^{\tau}$ is a collection of arbitrary polytopes, including convex or concave polytopes. We elaborate on the above integration for the case of two stochastic variables. In this case $A_{\Psi}^{\tau}$ is a set of polygons in the s-plane. By proper partitioning of each polygon $A_{\Psi}^{\tau}$, e.g., triangulation, one can rewrite Equation (6.2) as:

$$
\operatorname{Prob}^{\tau}(\Psi)=\int_{A_{2}} \int_{A_{1}\left(s_{2}\right)} d G_{1}\left(s_{1}\right) d G_{2}\left(s_{2}\right) .
$$

Now using the fact that the internal integral $\int_{A_{1}\left(s_{2}\right)} d G_{1}\left(s_{1}\right)=G_{1}^{\prime}\left(s_{2}\right)$ is a function of $s_{2}$ (since the area $A_{1}$ could be dependent on $s_{2}$ ), each multiple integration can be converted to a sequence of single definite integrations, for which robust and nearly exact numerical methods exist [79].

\subsubsection{Case studies}

\section{One general transition: scalability and efficiency}

The complexity and efficiency of measure computations clearly depend on the structure of the model. The process of computing measures of interest also linearly depends on the number of regions in the STD. In the following we experimentally show the scalability and efficiency of the proposed method when only one general transition is active in the system, using the case study in [26]. We scale the number of transitions 


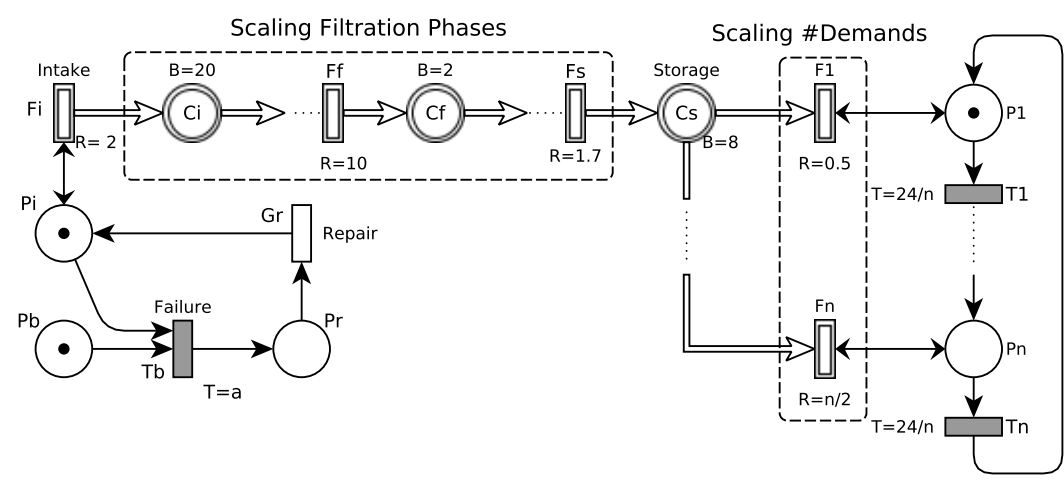

Figure 6.2.: HPnG model for an imaginary water treatment facility.

and continuous places in the case study, and discuss its influence on the number of regions and the computation time for the generation of the STD and the model checking.

Figure 6.2, presents a model of a water treatment facility with different phases. The continuous place $C_{i}$ represents the storage of a water softening phase. By design this is a slow process with large storage. The continuous place $C_{f}$ and transition $F_{f}$, represent a generic water filtering phase. Opposed to the softening phase this filtration phase is a fast process with small storage. The continuous place $C_{s}$, represents the final storage from which water is distributed to the customers with different rates, depending on the time of the day. The deterministic transition $T_{b}$ represents a failure at time $\alpha$ in the softening phase. When it fires, the continuous transition $F_{i}$ is disabled and the general transition $G_{r}$ becomes enabled. $G_{r}$ models the time it takes to repair the system failure according to the arbitrary probability density $g(s)$. Note that discrete place $P_{b}$ restricts the model such that the failure can occur only once.

The model presented in Figure 6.2 is made scalable in two ways. First, by cascading more filtration phases, and second by dividing the day into more intervals with different demand rates. In order to show the efficiency of our algorithm, we scale the model in these two ways, and for each instance, compute the probability distribution for the amount of fluid in place $C_{s}$. More specifically we compute the probability of $x_{C_{s}} \leq c$ by parametrizing $c$ from 0 to 8 (from being empty to full) with discretization step 0.2. This is an important measure of interest, because an empty final storage $C_{s}$ means failure to deliver water to the consumers. Moreover, we perform the analysis for a day, i.e., $t_{\max }=24$. All the computations have been performed on a machine equipped with a $2.0 \mathrm{GHz}$ intel $^{\mathbb{B}}$ CORE i7 processor, and $4 \mathrm{~GB}$ of memory. The results are shown in Tables 6.1 and 6.2. 


\begin{tabular}{c|ccc} 
\#Filters & \#Regions & STD $(\mathrm{ms})$ & MCT $(\mathrm{ms})$ \\
\hline 1 & 327 & 43 & 161 \\
2 & 433 & 80 & 239 \\
3 & 539 & 69 & 294 \\
4 & 663 & 77 & 373 \\
5 & 769 & 86 & 461 \\
6 & 903 & 95 & 509 \\
7 & 1026 & 106 & 586 \\
8 & 1159 & 121 & 662
\end{tabular}

Table 6.1.: Scaling the filtration phases. All times in milliseconds.

\begin{tabular}{c|ccc} 
\#Demands & \#Regions & STD $(\mathrm{ms})$ & MCT $(\mathrm{ms})$ \\
\hline 2 & 202 & 26 & 104 \\
3 & 403 & 76 & 202 \\
4 & 909 & 72 & 431 \\
5 & 1204 & 82 & 538 \\
6 & 1624 & 91 & 711 \\
7 & 1797 & 90 & 681 \\
8 & 2225 & 115 & 1004 \\
9 & 2776 & 125 & 1195 \\
10 & 3457 & 143 & 1451
\end{tabular}

Table 6.2.: Scaling the demand rates. All times in milliseconds.

Scaling in both dimensions increases the number of regions, as shown in the second column of both tables. The time needed to construct the STD is given in the third columns of both tables. The time needed to compute the measures of interest is denoted MCT (Measure Computation Time), and is given in the forth column of the tables. As can be seen scaling in any direction, with fixed maximum analysis time $\left(t_{\max }=24\right)$, does not cause the STD generation to take more than 200 millisecond. Moreover, although the measure computation is more time consuming than generation of STD, it only reaches a second for three last cases of scaling demand rates.

Note that once the STD is generated for a specific HPnG with a fixed initial state, it can be used for several measure computations. Therefore each row of the above tables corresponds to one STD generation and several computations of the probability of $x_{C_{s}} \leq c$ for different values of $c$. The same is true for different probability distribution, $g(s)$, associated with the general transition.

\section{Multiple General Transitions}

In this subsection we illustrate the feasibility of the proposed method for the case of multiple active general transitions. We investigate the survivability of a so-called GOOD (Given the Occurrence of Of Disaster) model, where the occurrence of a disaster is assumed at a certain point in time and the focus is on the recovery process, after that point in time. Figure 6.3, depicts the model of a water cleaning facility, where raw water is taken in via two entries $f_{1}$ and $f_{2}$ with rates 4 and 2 , respectively, and then cleaned in two separate cleaning streets. This set up increases the dependability of the system: in case the upper cleaning street fails, half of its input is rerouted to the lower cleaning street, which is then able to operate at a higher speed, modelled via the static fluid transition $f_{e}$ and the dynamic fluid transition $f_{4}$, respectively. However, since transition $f_{4}$ is handling twice of its normal load while the system is not repaired yet, it may fail as well. This is modelled by the general transition $T_{f}$. Note that the guard arc connecting $T_{f}$ and $D_{1}$ ensures that 
the failure can occur only if the repair process is not accomplished yet. Also note that, both the general transitions can fire only once, which is guaranteed through places $D_{1}$ and $D_{2}$.

We start with the initial state that the pump $f_{3}$ has failed and the repair process has been initiated (modelled by the general transition $T_{r}$, which is now enabled) and all the tanks are full initially. While the upper cleaning street is not repaired yet, there is a token in place $D_{1}$, transition $f_{e}$ is enabled and pumps additional water to the lower cleaning street at rate 2 . The rate of the dynamic transition $f_{4}$ is then equal to the sum of the rates of $f_{e}$ and $f_{2}$.

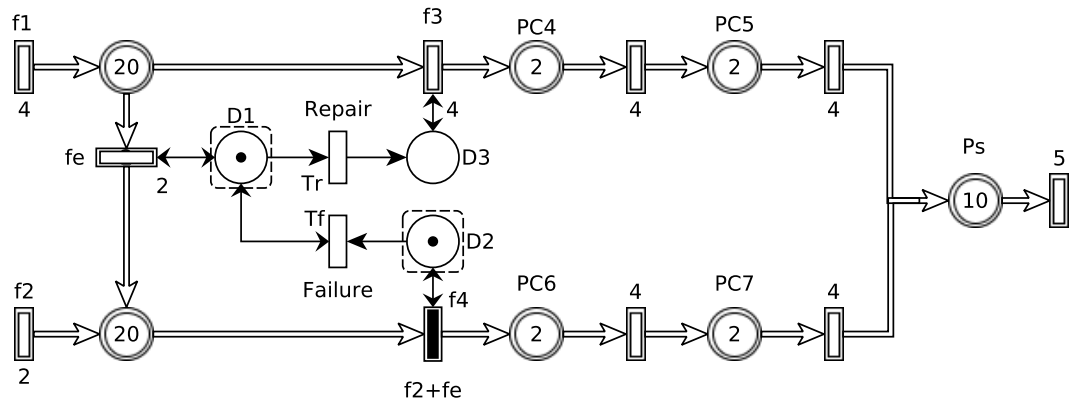

Figure 6.3.: HPnG model of a water treatment facility with two parallel cleaning streets.

Since we want to ensure that customers can reliably be served also in the presence of a failure in the system, we will compute the probability of having at least a certain amount of fluid in place $P_{S}$, which models a cleaned water storage, i.e.,

$$
x_{P_{S}} \geq b,
$$

for varying values of $b$. Figure 6.4 shows the probability that the above property holds at different times $\tau$ and for fluid levels, $b$, and different probability distribution for failure and repair, as provided in Table 6.3. The horizontal axis depicts the time of operation $(\tau)$, while the vertical axis is the probability of having more water in the reservoir $\left(x_{P_{s}}\right)$ than the given value, $b$ (color coded, see the legend, and each curve corresponds to a specific fluid level). In Figure 6.4a the repair process will take place earlier than the failure with a high probability (repair mean is 2, and failure mean is 6 hours). In contrast, Figure $6.4 \mathrm{~b}$, shows the results for a situation where a failure of the second cleaning street is likely to occur before the system is repaired (repair mean is 5, and failure mean is 2 hours). Finally, in Figure 6.4c the expected time of occurrence for repair and failure are almost at the same time, namely 3 and 4 hours after start time, respectively. 


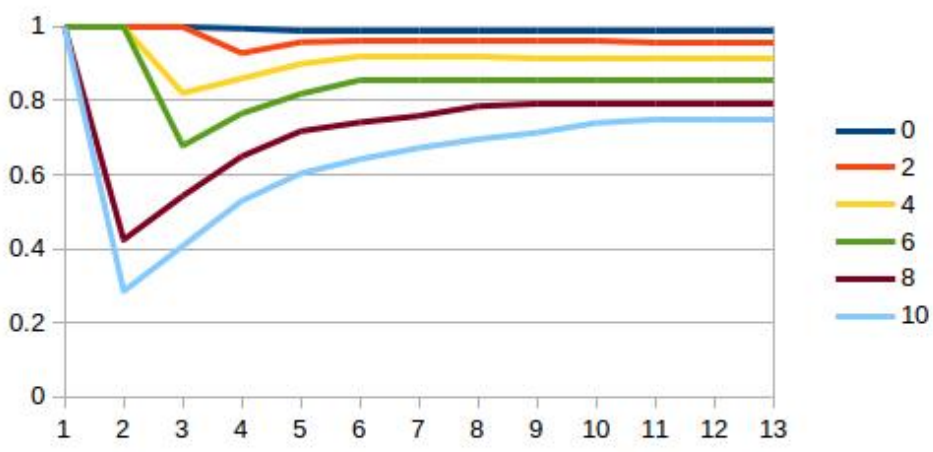

(a) Early repair.

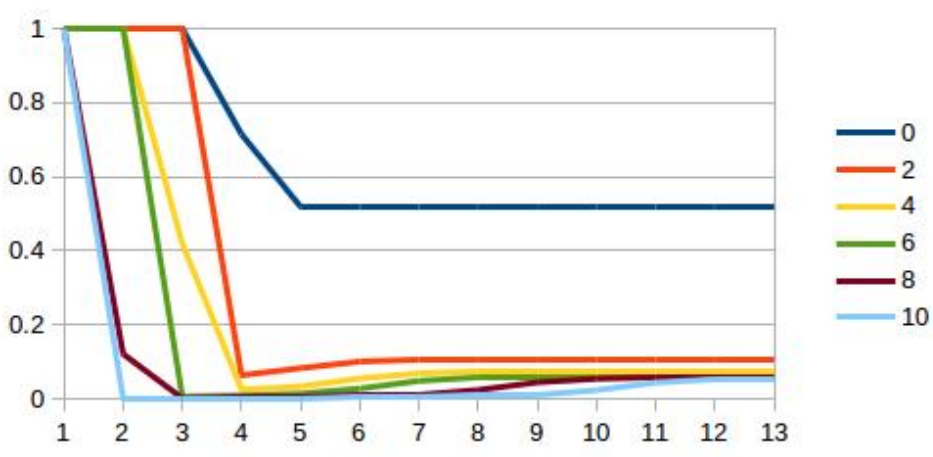

(b) Late repair.

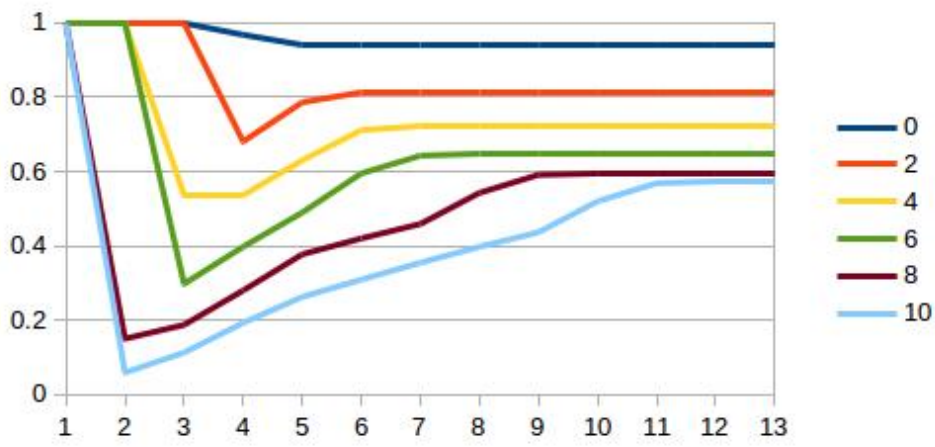

(c) When failure is likely to happen slightly after repair.

Figure 6.4.: Probability of having more than a given water level for a certain time. 
Table 6.3.: Different parameters of probability distributions.

\begin{tabular}{c|cc|cc} 
& \multicolumn{2}{|c|}{ Repair } & \multicolumn{2}{c}{ Failure } \\
\cline { 2 - 5 } & folded normal & mean & gamma & mean \\
\hline (a) & $\mu=2, \sigma=1$ & 2 & $k=3, \theta=2$ & 6 \\
(b) & $\mu=5, \sigma=1$ & 5 & $k=2, \theta=1$ & 2 \\
(c) & $\mu=3, \sigma=1$ & 3 & $k=2, \theta=2$ & 4
\end{tabular}

These figures show that the ability of the system to recover from the former disaster highly depends on the chosen probability distributions for repair and failure. In case (a) the system is highly survivable, since it quickly refills the water storage. However, the setting in case (b) cannot be considered survivable, as it reaches a predefined storage level only with a probability of approximately 0.1 . In case (c), where the expected time of failure to happen is slightly after the expected time of repair (4 and 3, respectively), the system recovers to a certain degree, however, a positive probability of keeping an empty final storage remains.

These experiments show how the analysis of Hybrid Petri nets with two general one-shot transitions helps to obtain insight in the recovery process of a system with stochastic failure and repair processes. Our analysis, in agreement with the common intuition, shows how important is the maintenance of the pump which receives the additional load after the first assumed failure. Since as can be seen, if this pump is expected to fail before the repair is being done, there is no possibility of recovery from the first failure.

The STD for this model has 85 regions, up to maximum time of one day $\left(t_{\max }=24\right)$, and its generation takes less than half a second. Each diagram in Figure 6.4, consists of 6 curves, each consists of 25 points. For each point a separate probability computation and integration needs to take place. In total, the generation of each curve has taken less than 5 seconds. All computations have been performed on a machine with a 2.o GHz intel ${ }^{\circledR}$ CORE i7 processor, and 4GB of memory.

\subsection{MODEL CHECKING UNTIL FORMULAS}

In this section we consider the general case of an STL formula, i.e., we focus mainly on model checking the Until operator. We emphasize that, due to the time-inhomogeneity of an HPnG, the validity of a certain formula depends on time. Model checking STL is based on computing the satisfaction sets as defined in Definition 4.5.

An STL formula without Until operator, as considered in the previous section, only depends on the current time of analysis and is independent of the further evolution of the system. Hence, it is enough to identify those regions where the system can be in at a given time $t$, and find out in which part of those regions the given formula is satisfied. In contrast 
to state-based properties, the validity of Until formula depends on the future system evolution. We have to find those areas of s-values for which the evolution of the system from a given starting time satisfies the formula.

Once we have these areas, in order to find the satisfaction probability of an Until formula, we can use the same integration methods given in Equations (6.1) and (6.2), for the case of one and multiple stochastic variables, respectively.

In this section we formalise the model checking algorithm for the case of multiple stochastic variables. However, for simplicity we illustrations the case of one active stochastic variable. In this case the areas of svalues will be reduced to intervals of $s$-value, and the satisfaction set in Definition 4.5 will be a set of intervals.

This section is further organized as follows. Section 6.2.1 introduces special notation for operations on families of sets that is needed in the algorithms. Section 6.2.2 explains the main idea of model checking the Until operator, and Section 6.2.3 presents the model checking algorithms in detail. In Section 6.2.4 we discuss the complexity of the model-checking an algorithm, and finally in Section 6.2.5, we provide a case study for the Until model-checking algorithm.

\subsubsection{Notation}

The algorithm presented in this section involves a special set of operations on sets of areas and on sets of system states. Hence, we introduce a new intersection and complement operator between families of sets.

Definition 6.1. Let $\mathcal{S}_{1}$ and $\mathcal{S}_{2}$ be two families of sets, the intersection operator between these two, and the complement operator of a family of sets, are defined as follows:

$$
\begin{array}{ll}
S \in \mathcal{S}_{1} \sqcap \mathcal{S}_{2} & \text { iff } \exists S_{1} \in \mathcal{S}_{1}, S_{2} \in \mathcal{S}_{2}: S=S_{1} \cap S_{2}, \\
S \in \neg \mathcal{S}_{1} & \text { iff } \forall S^{\prime} \in \mathcal{S}_{1}: \\
& S^{\prime} \cap S=\varnothing \wedge \nexists S^{\prime \prime} \in \neg \mathcal{S}_{1}: S^{\prime \prime} \subset S .
\end{array}
$$

According to the above definition we can derive the union and the relative complement operator as $\mathcal{S}_{1} \sqcup \mathcal{S}_{2}=\neg\left(\neg \mathcal{S}_{1} \sqcap \neg \mathcal{S}_{2}\right)$ and $\mathcal{S}_{1} \backslash \mathcal{S}_{2}=\mathcal{S}_{1} \sqcap \neg \mathcal{S}_{2}$, respectively. Intuitively, the union operator $\sqcup$, merges two families of sets and ensures that the results are maximal and there is no duplication (this is taken care of through the definition of complement). Also the intersection operator $\sqcap$, computes the intersection of all the set members of two given families of sets. For instance if we define two sets of intervals on real number as $\mathcal{S}_{1}=\{[1,3],[4,5]\}$ and $\mathcal{S}_{2}=\{[0,2],[3,5],[6,7]\}$, then we have, $\mathcal{S}_{1} \sqcap \mathcal{S}_{2}=\{[1,2],[4,5]\}$ and $\mathcal{S}_{1} \sqcup \mathcal{S}_{2}=\{[0,5],[6,7]\}$. These operations are also shown in Figure 6.5. ${ }^{1}$

1 Note that $[a, b]$ is the notation for an interval, which is indeed a dense set. 


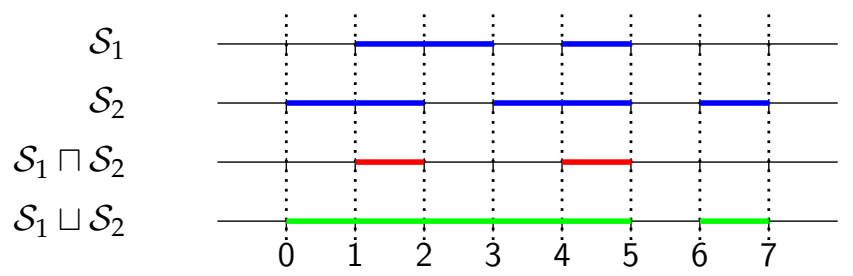

Figure 6.5.: Presentation of intersection and union operators over sets of intervals.

Finally, since the result of the algorithm are sets of satisfaction areas, we introduce a projection operator as follows:

Definition 6.2. The operator Prj projects a set of points $B$ in the $(\mathbf{s}, t)$-space on the s-space:

$$
\operatorname{Prj}(B)=\{\boldsymbol{s} \mid(s, t) \in B\} .
$$

If the given set is a connected set, the result of the projection is also a connected set. If $\mathcal{B}$ is a set of connected sets its projection results in a set of connected sets: $\operatorname{Prj}(\mathcal{B})=\bigsqcup_{B \in \mathcal{B}} \operatorname{Prj}(B)$. This operator is used later in the model checking algorithms.

\subsubsection{Model checking the until operator: preliminaries}

Recall that the evolution of the system for a fixed value of vector s, corresponds to a vertical line in the STD. The main idea behind checking formula $\Psi_{1} \mathcal{U}^{\left[T_{1}, T_{2}\right]} \Psi_{2}$ is to find all areas of s-values which satisfy $\Psi_{1}$ at the starting time. Then by moving upwards in time the STD, we refine the initial areas by omitting those parts which violate $\Psi_{1}$. We continue this until reaching either the time bound $T_{2}$ or a time point in $\left[T_{1}, T_{2}\right]$ for which $\Psi_{2}$ is satisfied. Due to the partitioning of the state space into regions, this can be done efficiently by traversing the regions in the STD. In order to model check the Until operator at the starting time $\tau$, for each possible value of vector s, i.e., different possible firing times of general transitions, we have to find a time point $t$ between $\tau+T_{1}$ and $\tau+T_{2}$, where $\Psi_{2}$ holds and before which $\Psi_{1}$ is not violated. Hence, the satisfaction set for a Until is defined as:

$$
\begin{aligned}
& \operatorname{Sat}^{\tau}\left(\Psi_{1} \mathcal{U}^{\left[T_{1}, T_{2}\right]} \Psi_{2}\right)= \\
& \bigsqcup\left\{A \subseteq \mathcal{S} \mid \exists t \in\left[\tau+T_{1}, \tau+T_{2}\right]:\right. \\
& \left.\quad A \models^{t} \Psi_{2} \wedge\left(\forall t^{\prime} \in[\tau, t]: A=^{t^{\prime}} \Psi_{1}\right)\right\} .
\end{aligned}
$$

The algorithm for model checking formula $\Psi_{1} \mathcal{U}^{\left[T_{1}, T_{2}\right]} \Psi_{2}$ at a given time $\tau$, is based on recursively traversing all regions in the underlying 
state space of an HPnG, starting from time $\tau$. Depending on the position of each region, relative to time $\tau$ and the interval $\left[T_{1}, T_{2}\right]$, we investigate (i) what are the areas in which $\Psi_{1}$ holds, and (ii) whether $\Psi_{2}$ holds in this region. This is explained in more detail in the following.

As sketched in Section 6.1.1, a discrete atomic property either holds in an entire region or not at all. However, atomic continuous properties need more care. Let $x(\mathbf{s}, t) \geq c$ be the continuous property, and recall that $x(\mathbf{s}, t)$ linearly depends on $\mathbf{s}$ and $t$. Then the property might be valid in only a part of the region. This part in the region can be indicated by intersection of half spaces specifying the boundary of the region and the half space characterized by $x(\mathbf{s}, t) \geq c$. Therefore, the half space $x(\mathbf{s}, t) \geq c$ splits the region in two parts, a part in which the property is valid and a part in which it is not valid.

Considering the linear boundaries of the region, the validity area of an atomic property in a region is a polytope. The validity area of the conjunction of two continuous atomic properties is the intersection of the corresponding polytopes, and the negation results in the complement of the validity area with respect to the considered region. Hence, in order to find the validity area of a formula, which consists of negation and conjunctions of atomic properties, first we need to form the parse tree of the formula. Then, by repeated application of the respective operators the validity area is formed. Moreover, if we consider a polytope as a set of points, then any set theoretical operation between two polytopes, results in a set of polytopes (convex or non-convex). Therefore, the validity area of a formula $\Psi$ is also a set of polytopes.

More formally, denote the set of polytopes in region $R$, in which formula $\Psi$ is satisfied by $\mathcal{P}_{\Psi}(R)$. Then, for the state-based STL formula (i.e., Until operator excluded) the validity areas are defined as follows:

Definition 6.3. The set of polytopes within region $R$, where a state-based $S T L$ formula holds is given by:

$$
\begin{aligned}
\mathcal{P}_{m_{P}=a}(R) & =\left\{(s, t) \in R \mid \Gamma(s, t) \cdot m_{P}=a\right\}, \\
\mathcal{P}_{x_{P} \geq a}(R) & =\left\{(s, t) \in R \mid \Gamma(s, t) \cdot x_{P} \geq a\right\}, \\
\mathcal{P}_{\Psi_{1} \wedge \Psi_{2}}(R) & =\mathcal{P}_{\Psi_{1}}(R) \sqcap \mathcal{P}_{\Psi_{2}}(R), \\
\mathcal{P}_{\neg \Psi}(R) & =R \backslash \mathcal{P}_{\Psi}(R) .
\end{aligned}
$$

Note that if $\Psi$ is an atomic property, then $\mathcal{P}_{\Psi}(R)$ is a single polytope. Figure 6.6, schematically, shows the validity areas of two properties $\Psi_{1}$ and $\Psi_{2}$ in a hypothetical region, for the case of only one stochastic variable. This figure illustrate arbitrary nature of validity areas, as they can be non-convex, and unconnected. For intance, as can be seen, the area corresponding to $\Psi_{1}$ is a non-convex polygon, and the area satisfying $\Psi_{2}$ consists of two polygons. 


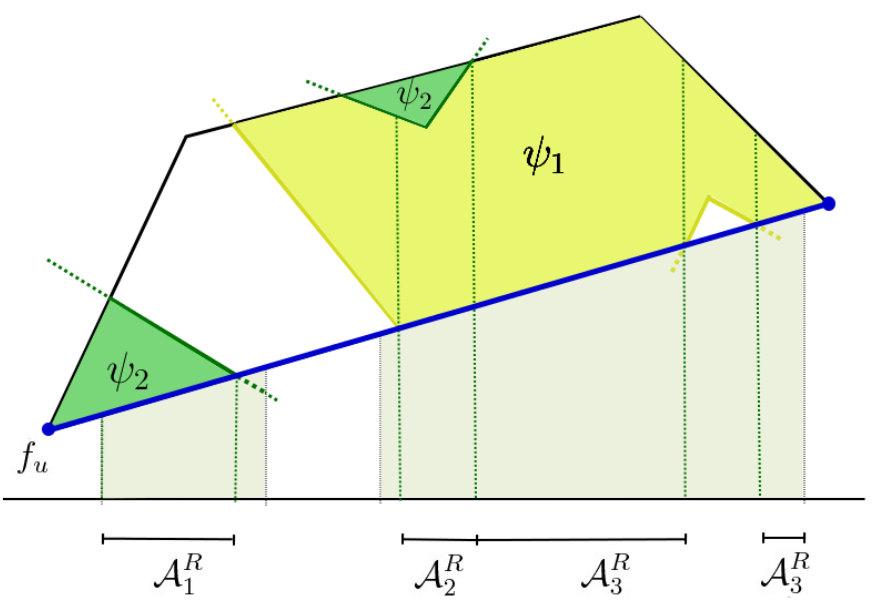

Figure 6.6.: Determining intervals that satisfy formula $\Psi_{1} \mathcal{U}^{\left[T_{1}, T_{2}\right]} \Psi_{2}$, in a generic region.

\subsubsection{Model checking the until operator: algorithm}

The procedure for model checking the Until formula, $\Psi_{1} \mathcal{U}^{\left[T_{1}, T_{2}\right]} \Psi_{2}$, at a given time $\tau$, is presented in Algorithm 6.1. Note that $\Psi_{1}$ and $\Psi_{2}$ can be negation and conjunction of any atomic formulas. At first we determine all starting regions, i.e., all regions we can be in at time $\tau$. This is done by finding the set of regions, $\mathcal{R}_{\tau}$, intersecting with hyperplane $t=\tau$. Then we iterate over all these regions and determine the areas in s-space which satisfy the Until formula, by calling the recursive function VISIT for each region. This function is the core of the model checking procedure and is presented in Algorithm 6.2.

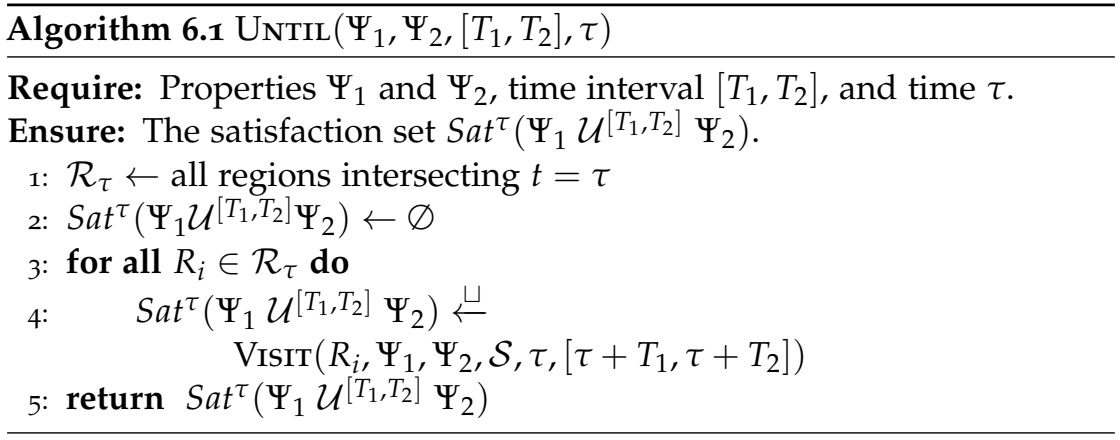

Function VIsIT takes as input the region to be visited, denoted $R$, formulas $\Psi_{1}$ and $\Psi_{2}$, starting time $\tau$ and interval $\left[T_{1}, T_{2}\right]$ of the Until for- 
mula. Furthermore, it receives the set of areas $\mathcal{A}_{\Psi_{1}} \subseteq \mathcal{S}$, which satisfy $\Psi_{1}$ just before entering region $R$. Hence, $\mathcal{A}_{\Psi_{1}}$ is the set of potential areas in s-space which may satisfy the Until formula. This set is determined based on the regions which are the predecessor to the region being visited, as will be explained below. For the initial call of VISIT, this set contains the biggest area in which the Until formula may hold, which is $\mathcal{S}$.

At first (lines 2-3) we form the set of polytopes corresponding to $\Psi_{1}$ and $\Psi_{2}$, as defined in Definition 6.3. Then, for each region we have to find three sets of areas in s-space:

(i) $\mathcal{A}_{1}^{R}$ : the set of areas which satisfy the Until formula upon entering the region, because upon arrival $\Psi_{2}$ is satisfied;

(ii) $\mathcal{A}_{2}^{R}$ : the set of areas that satisfy $\Psi_{1}$ and reach a $\Psi_{2}$ polytope in the region in time, therefore they also satisfy the Until formula;

(iii) $\mathcal{A}_{3}^{R}$ : the set of areas in which $\Psi_{1}$ holds, but a $\Psi_{2}$ polytope cannot be reached in time within the region. These are the areas for which the successive regions need to be considered to check whether they reach a $\Psi_{2}$ polytope in the future (but within the time bound).

These three sets of areas are illustrated in Figure 6.6, for the case of one stochastic variable. Note that in this case areas are reduced to intervals on the s-axis. Below we discus the characterization and construction of these areas in details .

As pointed out before, the position of a region with respect to the starting time $\tau$, and interval $\left[T_{1}, T_{2}\right]$ should be taken into account during the computation. If a region intersects the hyperplane $t=\tau$, only the part above this hyperplane needs to be considered, i.e., the halfspace $t \geq \tau$. If a region is above the line $t=\tau$, but below the hyperplane $t=T_{1}$, according to the definition of the Until operator, we only need to consider those areas in s-space, which satisfy $\Psi_{1}$. If a region intersects the hyperplane $t=T_{1}$, we have to split it into two parts. The part below this hyperplane only needs to be considered if $\Psi_{1}$ holds, and for the upper part we have to apply the general algorithm, as explained before. The same applies, if the region is between the hyperplane $t=T_{1}$ and $t=T_{2}$. However, for simplicity, Algorithm 6.2, only discusses the general case where the region is placed inside the interval $\left[T_{1}, T_{2}\right]$. The other cases are the easily the subset of the operations being done in this general case.

First set of areas: $\mathcal{A}_{1}^{R}$

For finding the first area set, $\mathcal{A}_{1}^{R}$, we have to check if the polytopes that form the validity area of $\Psi_{2}$ (Definition 6.3) intersect the underlying facet of the region $R$ (as defined in Section 5.2.2), denoted R. $f_{u}$ (and shown 


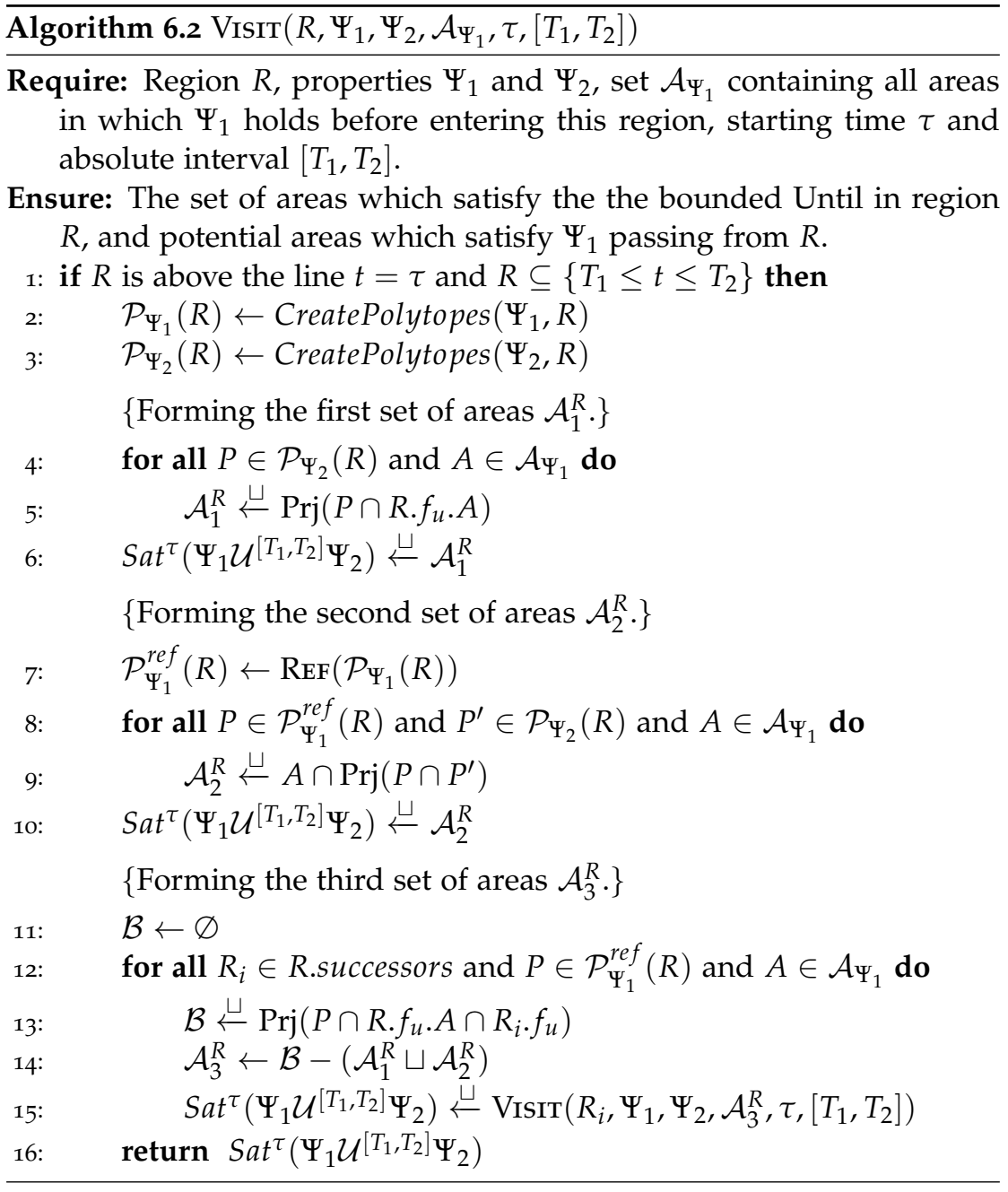

in Figure 6.6), and whether the intersection (or part of it) lies in one of the areas that belongs to $\mathcal{A}_{\Psi_{1}}$. More formally, we denote the part of the underlying facet in which $\Psi_{1}$ holds, i.e., the part which lies in $\mathcal{A}_{\Psi_{1}}$ as

$$
R . f_{u} \cdot \mathcal{A}_{\Psi_{1}}=\left\{(\mathbf{s}, t) \in R \cdot f_{u} \mid \exists A \in \mathcal{A}_{\Psi_{1}}, \mathbf{s} \in A\right\} .
$$

Then, the first area set is computed as the projection of the intersection of the validity area of $\Psi_{2}$ and the $\Psi_{1}$ part of the underlying facet:

$$
\mathcal{A}_{1}^{R}=\operatorname{Prj}\left(\mathcal{P}_{\Psi_{2}}(R) \sqcap R \cdot f_{u} \cdot \mathcal{A}_{\Psi_{1}}\right) .
$$

This is done in lines 4-6 of Algorithm 6.2, and illustrated in the left part of Figure 6.6 for the case of one stochastic variable. As can be seen, a $\Psi_{2}$ polytope intersects the underlying facet in one of the areas 
which satisfies $\Psi_{1}$. Hence, the resulting intersection areas satisfy the Until formula, therefore it is merged inside the satisfaction set.

Note that, since the polytopes corresponding to an STL formula are not necessarily convex, they may have several intersections with the underlying facet. We emphasize again in case of Figure 6.6, since there is only one stochastic variable, polytopes are polygons, facets are segments, and areas on s-space are intervals.

Second set of areas: $\mathcal{A}_{2}^{R}$

The next step is to find the second set of areas, $\mathcal{A}_{2}^{R}$. For this first the set of $\Psi_{1}$ polytopes $\mathcal{P}_{\Psi_{1}}(R)$ needs to be computed. However, since the polytopes are not necessarily convex, we only consider that part of the region where $\Psi_{1}$ continuously holds over time. This is illustrated in Figure 6.7, where for any value of $s_{1}<s<s_{2}, \Psi_{1}$ may be violated, since part of the evolution lies outside the depicted $\Psi_{1}$ polytope. For instance for the depicted value of $s=s^{*}, \Psi_{1}$ does not hold between time $t_{1}$ and $t_{2}$.

Hence, the polytopes are reformed to ensure that the $\Psi_{1}$ polytope only contains system states $\Gamma(\mathbf{s}, t)$ where in the respective polytope the evolution until time $t$ continuously satisfies $\Psi_{1}$. In other words, we make the polytopes convex with respect to the vertical lines. More specifically this means for any vertical line, if two points on this line lies inside the polygon, all the points between them also lies inside the polygon. For this the operator REF over a polytope $P$ is introduced as follows:

$$
\begin{gathered}
(\mathbf{s}, t) \in \operatorname{REF}(P) \Leftrightarrow \\
\forall\left(\mathbf{s}, t^{\prime}\right) \in P, t^{\prime} \leq t: \nexists t^{\prime \prime} \in\left[t^{\prime}, t\right]:\left(\mathbf{s}, t^{\prime \prime}\right) \notin P .
\end{gathered}
$$

A reformed polytope is illustrated in Figure 6.7, for a case of one stochastic variable, by shaded blue area. Then $\mathcal{A}_{2}^{R}$ can be computed by projecting the intersection of the reformed $\Psi_{1}$ polytope with the $\Psi_{1}$ part of the underlying polytope, i.e., $R \cdot f_{u} \cdot \mathcal{A}_{\Psi_{1}}(R)$, and with a $\Psi_{2}$ polytope. The resulting intersection areas then satisfy the bounded Until formula:

$$
\mathcal{A}_{2}^{R}=\operatorname{Prj}\left(\operatorname{ReF}\left(\mathcal{P}_{\Psi_{1}}(R)\right) \sqcap R \cdot f_{u} \cdot \mathcal{A}_{\Psi_{1}} \sqcap \mathcal{P}_{\Psi_{2}}(R)\right) .
$$

This is done in lines 7-10 of Algorithm 6.2. First all the $\Psi_{1}$ polytopes are reformed. Then we iterate over all reformed $\Psi_{1}$ polytopes, the set of $\Psi_{2}$ polytopes and the set of areas in $\mathcal{A}_{\Psi_{1}}$, and add their intersection to the second area set i.e., $\mathcal{A}_{2}^{R}$. Finally, this set is also merged inside the satisfaction set, since it satisfies the Until formula.

Third set of areas: $\mathcal{A}_{3}^{R}$

We also need to consider those area in the s-space, through which we can enter and leave a region without violating $\Psi_{1}$, which corresponds to 


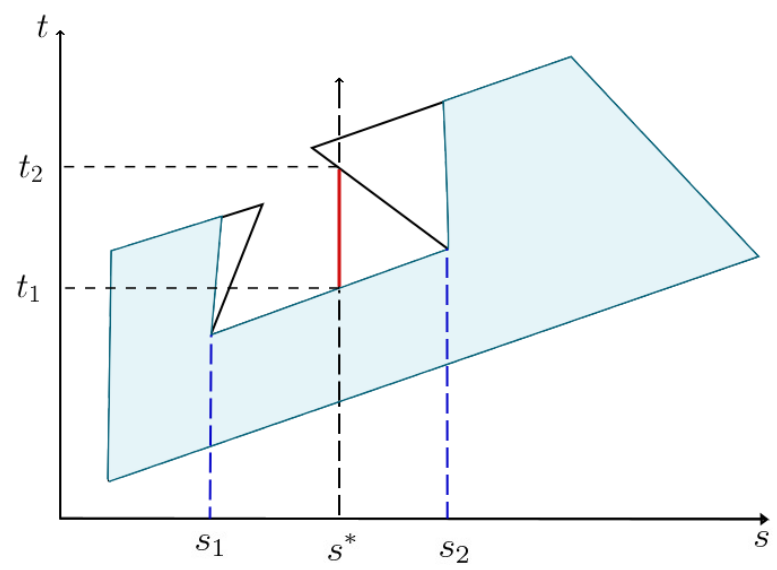

Figure 6.7.: Reforming a polygon over a given interval.

the third category of areas mentioned earlier. This is done in lines 11-15 of Algorithm 6.2. We iterate over all possible successors of the region $R$ and check for each successor region whether a reformed $\Psi_{1}$ polytope intersects with its underlying facet, in order to find all potential areas that can be used to enter the new region. However, only those areas that have non-empty intersection with the former set $\mathcal{A}_{\Psi_{1}}$ are valid. We also only consider those areas which have not already satisfied the overall Until formula. The latter point is considered in line 14, by subtracting the two computed sets $\mathcal{A}_{1}^{R}$ and $\mathcal{A}_{2}^{R}$. Finally, in line 15 , we call the VISIT function recursively for the successive regions, with $\mathcal{A}_{3}^{R}$, as input for the potential satisfaction set.

\subsubsection{Complexity}

In this section we presented a detailed algorithm for model checking time-bounded Until properties on HPnGs. The algorithm is based on grouping, per region, all system states that satisfy a given STL formula into sets of polygons. Note that, due to negation and conjunction operations, the resulting validity area is not necessarily a convex polygon. In computational geometry this problem is referred to as polygon clipping [8o-82], and there is robust implentation of that in CGAL (Computational Geometry Algorithm Libray) [77,83]. The worst case complexity for finding intersection of two polygons is $O(n \cdot m)$, in which $n$ and $m$ are the number of polygons edges. Note that the number of edges of polygons in our algorithm corresponds to the number of continuous atomic properties in the given STL formulas.

In the worst case in order to find the final validity area for a formula, we may have to visit all regions. Therefore, the final complexity of the 
algorithm for model checking a formula is dependent on the number of regions multiplied with the number of continuous atomic properties. The number of regions in the STD depends on both the structure and the initial marking of the HPnG. More precisely, the way that model components are connected to each other and the initial distribution of tokens and fluid levels in discrete and continuous places, determine the number of regions. This means that a priori bound on the number of regions based on only the network structure is not possible since for different initial states we may have different number of regions. Therefore, the number of regions in a STD cannot be determined a priori.

\subsubsection{Case study: Model Checking Until operator}

In this section we reconsider a simplified version of the case study as in Section 6.1.3, to investigate true survivability properties, which previously was not possible. This is depicted in Figure 6.9. Here again we use a so-called Given the Occurrence Of Disaster (GOOD) model in a way that we assume that a failure (disaster) has just happened. This allows us to evaluate the recovery process and study the impact of the disaster on the system operation.

The model consists of a water storage tank (place $C_{S}$ ) with a constant inflow at rate 1.7 (transition $F_{S}$ ) and an outflow during day with rate 2 units/h (transition $F_{d}$ ) between 6:00 and 21:00, and a lower outflow during night, with rate 1 units / $h$ between 21:00 and 6:00 the next day. The day-night pattern is governed by transitions $T_{n}$ and $T_{d}$.

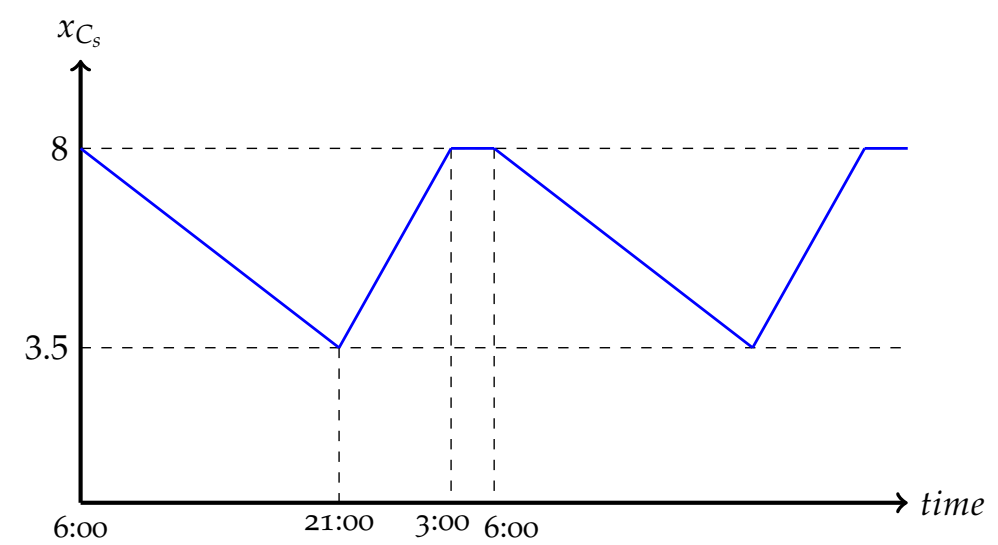

Figure 6.8.: Evolution of content of place $C_{s}$ over time, in normal condition, i.e., no failure occurred.

Under normal operation the amount of water in the storage fluctuates between 3.5 and 8 units and stays at its maximum between 3:00 and 6:00. 
During this time span the inflow is reduced to 1 units/ $h$ in order to match the outflow and to prevent the storage from overflowing, due to rate adaptation process (refer to Section 3.5). Figure 6.8, illustrated the evolution of content of the storage over time in normal operation. By introducing a single failure, occurring at different times of the day, we turn off the inflow to the tank, via the firing of the deterministic transition $T_{b}$. The repair period that follows has stochastic length, modelled by transition $G_{r}$, of which the duration can follow any possible distribution.

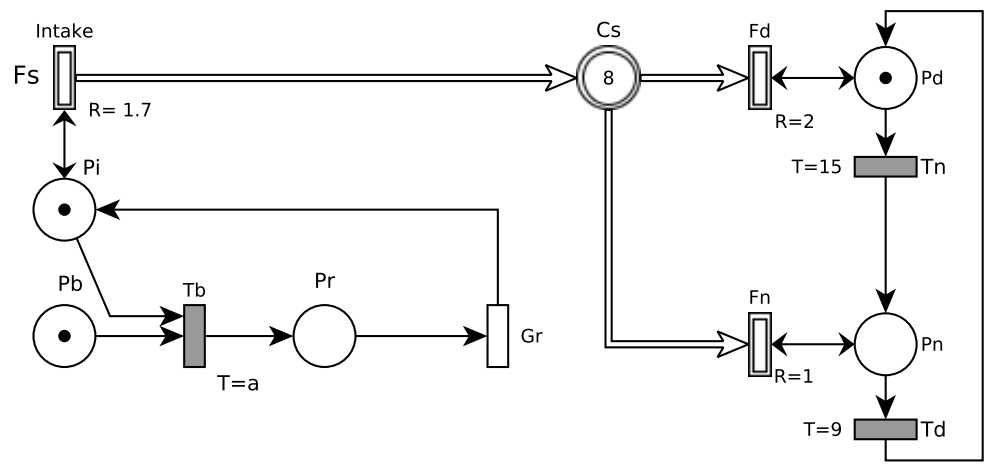

Figure 6.9.: Model of a water storage with different demands during night and day.

To analyze the survivability of the system, we check whether the system recovers within a time bound $T$, after the occurrence of the disaster, to a predefined service level without violating a preset safety condition with probability at least 0.7 . The system is defined as being recovered when the storage holds at least 3 units of water and the inflow is restored, i.e., the discrete place $P_{i}$ holds a token. We require that during the recovery process the amount of water never drops below 0.1 units, which can be seen as a safety threshold. This translates to checking whether the following formula, expressing the system survivability, holds:

$$
\mathbb{P}_{\geq 0.7}\left(\left(x_{C_{s}} \geq 0.1\right) \mathcal{U}^{[0, T]}\left(x_{C_{s}} \geq 3 \wedge m_{P_{i}}=1\right)\right) .
$$

Figure 6.10 shows the probability for the system to recover (the probability of holding above formula) before a certain time bound $T$ (depicted on the $x$-axis), for four different failure occurrence times. The repair process is assumed to follow an exponential distribution with mean 3 . Note that we start the model checking at the time of failure, so due to the definition of the Until operator, time on the horizontal axis is relative to the failure occurrence time. As can be seen in the figure, the survivability of the system highly depends on the time of the occurrence of the failure; a failure which occurs later during the day recovers with smaller probability. This is due to the fact that during the day the buffer is emptied, 


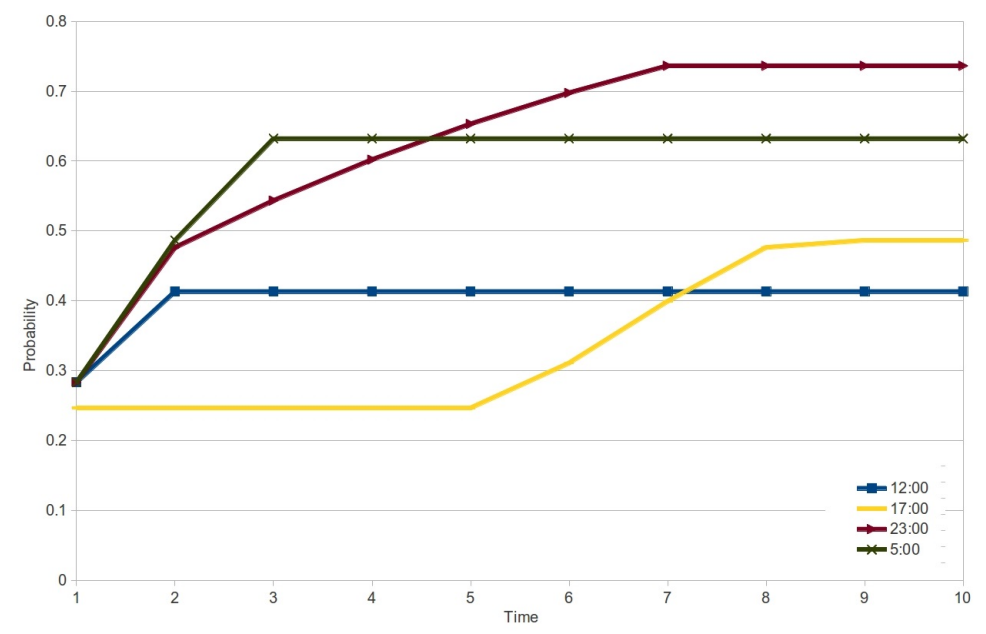

Figure 6.10.: Survivability probability for different failure times with negative exponential distributed repair $($ mean $=3)$.

hence, the probability to hit the safety threshold in the storage becomes larger. However, in case the failure occurs during the night, the survivability is higher, since the time window between 21:00 and 24:00 is used to refill the storage. Note that, for the presented occurrence times of failure the probability to recover is only larger than $70 \%$ when the failure occurs at 23:00; hence, the overall formula is not generally satisfied.

Figure 6.11 shows the probability for the system to recover for different repair distributions, namely, Gamma, Normal and Exponential distributions. Note that the Normal distribution is truncated for the values smaller than zero and renormalized, and we use the Gamma distribution with shape parameter 4 , and scale parameter 0.5. All the distributions have the same mean of 2 hours, but with different variances. As can be seen, the exponentially distributed repair time yields a lower probability for the survivability property to hold than the Normal and Gamma-distributed repairs. Figure 6.11 clearly shows the importance of being able to model general transitions with different distributions.

For computing the probabilities, according to Definition 4.6, the model checking algorithm first computes the set of satisfaction intervals $\operatorname{Sat}^{t}(\Psi)$. Here these intervals can be interpreted as the maximum available time window for repair, such that the survivability condition is not violate. Table 6.4 summarizes these intervals for the four different failure occurrence times and six different time bounds. For instance, for a failure occurrence time of 23:00, these time-dependent intervals are $(0,2.76)$ and $(0,3.58)$ for $4 \mathrm{~h}$ and $6 \mathrm{~h}$, respectively. This means that, in case of occurrence of failure at time 23:00, the system should be repaired in less 2.76 hours if we want to be sure that the system is going to recover $\left(x_{C_{s}} \geq 3\right)$ 


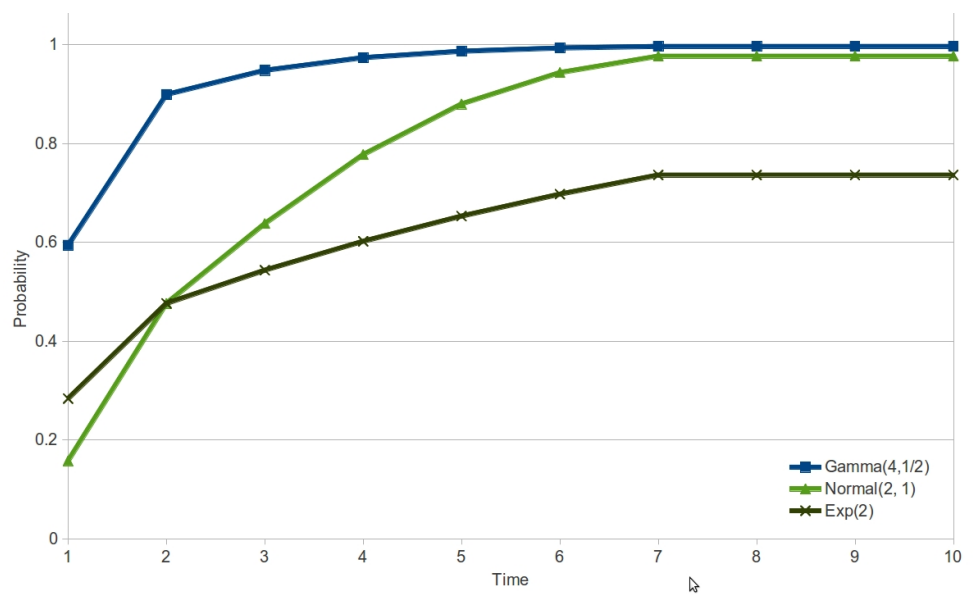

Figure 6.11.: Survivability probability for different repair distributions for a failure at 23:00

in 4 hours without violating safety condition $\left(x_{C_{s}} \geq 0.1\right)$. This is indeed a very useful information for scheduling repairs.

Table 6.4.: Satisfaction intervals for different failure times (leftmost column) and time bounds for repair (columns 2-6)

\begin{tabular}{r|cccccc}
$\mathrm{T}$ & $\mathrm{Th}$ & $2 \mathrm{~h}$ & $4 \mathrm{~h}$ & $6 \mathrm{~h}$ & $8 \mathrm{~h}$ & $1 \mathrm{oh}$ \\
\hline $5: 00$ & $(\mathrm{o}, 1)$ & $(\mathrm{o}, 2)$ & $(\mathrm{o}, 3)$ & $(\mathrm{o}, 3)$ & $(\mathrm{o}, 3)$ & $(\mathrm{o}, 3)$ \\
$12: 00$ & $(\mathrm{o}, 1)$ & $(\mathrm{o}, 1.6)$ & $(\mathrm{o}, 1.6)$ & $(\mathrm{o}, 1.6)$ & $(\mathrm{o}, 1.6)$ & $(\mathrm{o}, 1.6)$ \\
$17: 00$ & $(\mathrm{o}, 0.85)$ & $(\mathrm{o}, 0.85)$ & $(\mathrm{o}, 0.85)$ & $(\mathrm{o}, 1.12)$ & $(\mathrm{o}, 1.94)$ & $(\mathrm{o}, 2)$ \\
$23: 00$ & $(\mathrm{o}, 1)$ & $(\mathrm{o}, 1.94)$ & $(\mathrm{o}, 2.76)$ & $(\mathrm{o}, 3.58)$ & $(0,4)$ & $(0,4)$
\end{tabular}

One can use these satisfaction set of intervals to schedule optimal repair strategies. For instance the Gamma distribution has most of its probability mass in the corresponding intervals, hence, yields the highest survivability. This is depicted in Figure 6.12. It can be seen, that the probability mass accumulated in the interval $(0,2.76)$ is smaller for the Exponential distribution comparing to the Normal and, the Gamma distribution. This specifically is observable in Figure 6.12b, in which CDF functions are shown.

To obtain the results presented in this case study the algorithms have been been executed on a machine equipped with a $2.0 \mathrm{GHz}$ intel ${ }^{\circledR} \mathrm{CORE}$ i7 processor, $4 \mathrm{~GB}$ of memory. Computation times, for generation of STD and investigating survivability, vary between $10 \mathrm{~ms}$ and $50 \mathrm{~ms}$, depending on the time bound. 


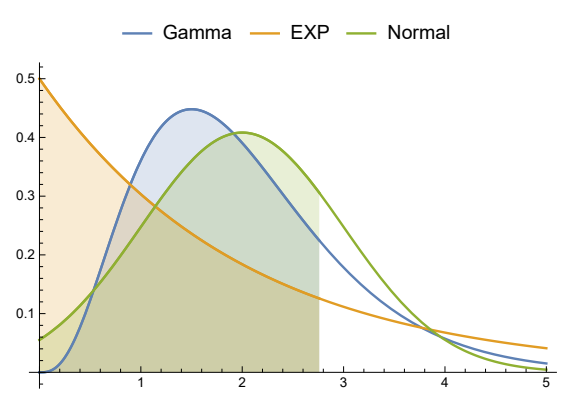

(a) PDFs.

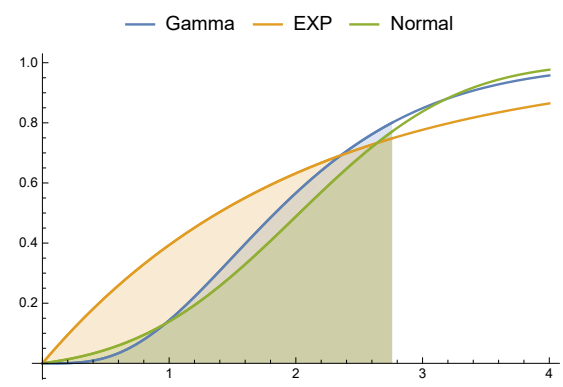

(b) CDFs.

Figure 6.12.: Representation of probability mass on the interval $(0,2.76)$, for three different probability distributions.

\subsection{RELATED WORK}

For model checking linear hybrid systems, different approaches exist, such as linear abstraction, used in HyTech/PHAVer [84, 85] and flow pipe construction, as used, e.g., in SpaceEx [86]. However, our work differs from these approaches, since we partition the state-space according to time and the support of stochastic variables, instead of the values of continuous variables. Hence, regions presented in this dissertation do not correspond to modes in hybrid automata. Due to the partitioning of the state-space into regions with the same characteristics, we are able to provide exact results for time-bounded reachability within few seconds.

This considerably differs from related work on Stochastic Hybrid systems (with deterministic continuous behaviour), where mostly over approximation $[76,87,88]$ is used to compute results. The practical applicability of these approaches, however, seems to be limited to special cases. Another option is to use Monte-Carlo simulations, as done in $[89,90]$.

For model checking Fluid Stochastic Petri nets [91] two comments are in place. First, the model class is different, since their analyzability is restricted to only a few fluid places. Second, we are not aware of a unifying approach that incorporates both discrete and continuous properties, in a same logic, as we do in STL. However, model checking of temporal logics in a restricted class of probabilistic hybrid automata, i.e., a hybrid automata with discrete probabilistic jumps, has been shown to be decidable [14]. In the next chapter we discuss this work in more details.

Finally at the end of this section we provide a comparison between model checking using STDs and parametric reachability method [26] which was briefly discussed at the end of Section 5.3. We do consider this method specifically because the idea of conditioning the state space 


\begin{tabular}{|c|c|c|c|c|c|c|c|c|c|}
\hline & \multicolumn{2}{|c|}{ Region based } & \multicolumn{2}{|c|}{ Param. reach. } & & \multicolumn{2}{|c|}{ Region based } & \multicolumn{2}{|c|}{ Param. reach. } \\
\hline \#Filters & STD & MCT & Tree & MCT & \#Demands & STD & MCT & Tree & MCT \\
\hline 1 & 43 & 161 & 10 & 11106 & 2 & 26 & 104 & 15 & 32348 \\
\hline 2 & 80 & 239 & 19 & 13153 & 3 & 76 & 202 & 21 & 43874 \\
\hline 3 & 69 & 294 & 19 & 15415 & 4 & 72 & 431 & 23 & 52526 \\
\hline 4 & 77 & 373 & 22 & 17188 & 5 & 82 & 538 & 38 & 66793 \\
\hline 5 & 86 & 461 & 25 & 19352 & 6 & 91 & 711 & 49 & 79479 \\
\hline 6 & 95 & 509 & 26 & 21501 & 7 & 90 & 681 & 30 & 69484 \\
\hline 7 & 106 & 586 & 30 & 23385 & 8 & 115 & 1004 & 99 & 115542 \\
\hline 8 & 121 & 662 & 31 & $\begin{array}{l}23505 \\
25875\end{array}$ & 9 & 125 & 1195 & 102 & 120129 \\
\hline & & & & & 10 & 143 & 1451 & 133 & 136896 \\
\hline
\end{tabular}

Table 6.5.: Scaling the filtration phases. Table 6.6.: Scaling the demand rates. All All times in milliseconds. times in milliseconds.

on the support of general transitions is introduced with this method. For this we consider the first case study of Section 6.1.3, given in Figure 6.2. We compute the same measures of interest as described for the mentioned case study using parametric reachability. The comparison results is given in Tables 6.5, 6.6, juxtaposing the computation times using both STDs and parametric reachability.

For both methods two computation times are given. The first one is the time for generation of the state space, which is the generation of the STD for the method introduced in this thesis, and parametric location tree, for parametric reachability method. The second computation time is for computing the same measure of interest for both methods (the probability of $x_{C_{s}} \leq c$ by parametrizing $c$, as described for case study of Figure 6.2). This is abbreviated as MCT for Measure Computation Time.

As one can see, when scaling the number of filters the generation of the STD takes about 3 to 4 times longer than the construction of the parametric tree. This is due to the more involved computations that are necessary to construct the polygons in the STD. When scaling the number of demands the generation of the STD takes about 2 to 3 times longer than the construction of the parametric locations. This is, however, more than compensated for when the measures of interest are computed. Model checking using STDs, depending on the size of the model, is between 20 and 100 times faster than the parametric reachability method. Moreover, as can be seen, for smaller models the speed up is larger than for bigger models. This is because the complexity of the old algorithm is logarithmic in number of parametric locations.

As mentioned in Section 5.3, the time for computing measures for the parametric reachability algorithm depends on the chosen discretization step. The results presented in the tables have been obtained for a discretization step of 0.005 . A larger discretization step reduces the MCT, but also decreases the accuracy of the results. For a discretization step of 0.005 , the maximum difference between the results from both algorithms is $0.5 \%$. Running the parametric location algorithm with a discretization 
step of 0.2 leads to approximately the same MCT with both algorithms, the resulting maximum relative error, however is $3 \%$.

In conclusion, in order to compare both methods, one should consider both computation times, i.e., the one regarding generation of state space (STDs and parametric location trees), and the one related to computation of measures of interest. The former is smaller for parametric reachability method (especially for very complicated HPnGs), and the latter is smaller for region based method. However, one should note that once the state spaces is generated, several measure computations may take place. Hence, if several properties for a system needs to be investigated, region based method is favourable. Having said this, we should also mention that in the example case study considered here (Figure 6.2) we have considered one specific network structure, i.e., the scaling is done by serializing continuous places, and continuous transitions. It is possible that for other network structures, e.g., parallelizing instead of serializing, the computation time for generation of the state space for region based method increases more than what is observed here. Therefore, there may be cases that parametric reachability method be favourable in overall. At the end, we have to stress the fact that parametric reachability method, is an approximation techniques, and we will discuss approximation techniques built upon region based method in the next chapter. 



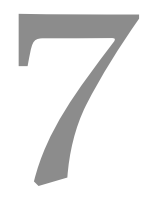

\section{APPROXIMATION TECHNIQUES}

The content of this chapter is based on:

[92] H. Ghasemieh, A. Remke, and B. R. Haverkort. Survivability analysis of a sewage treatment facility using hybrid Petri nets. Performance evaluation, 97:36-56, 2016

[93] H. Ghasemieh, A. Remke, B. R. Haverkort, and G. Ciardo. Approximate analysis of hybrid Petri nets with probabilistic timed transitions. In 9th International Conference on Performance Evaluation Methodologies and Tools, pages 127134. ICST, 2016.

In previous chapters we proposed methods for computing exact probabilities for various measures of interests. However, as we saw, the proposed methods are limited in the number of stochastic variables they can handle. In this chapter we will tackle this limitation, by confining ourselves to approximation of probabilities instead of their exact computation. For this purpose we introduce approximation techniques.

We will consider two methods. Both methods are based on discretizing the firing times of general transitions, i.e., confining the support of one or more stochastic variables to finite sets. In the first approach, we allow one stochastic variable with dense support as we had so far, and will discretize the support of the remaining stochastic variables. In this chapter we confine ourselves to only theoretical aspects of this approach and provide a simple example. In Chapter 8, we will investigate and discuss in more details the implications of using this method.

For the second approach we limit the support of all stochastic variables to only finite sets (unlike the previous method in which one of them was allowed to have dense support). Assuming a finite support for stochastic variables instead of dense sets, permits us to undertake a completely different solution method. More specifically, the state space of the system will collapse to a tree like structure and finding the satisfaction set is reduced to well-known tree search problems.

This chapter discussed these two approaches in two main sections. Section 7.1 discusses the discretization of all stochastic variables but 
one. Section 7.2, investigates the approach of limiting the support of all stochastic variables to finite sets, and provides a case study to show its feasibility. In Section 7.3, the new methods are compared with each other. Finally, Section $7 \cdot 4$, discusses related work.

\subsection{DISCRETIZATION}

In this section we propose to discretize the firing times of all general transitions, except for one. The idea is to select a set of firing times (discretization points) for each general transition and treat them as deterministic transitions for those firing times, and generate an STD for the remaining general transition (the one left to be continuous), for each of these discretization points.

\subsubsection{Approach and algorithm}

We discuss the algorithm for the case of only two stochastic variable. Like before, let $S_{1}$, and $S_{2}$ be the real valued random variable representing the firing time of the first and the second general transitions, i.e., the time that they will fire relative to their enabling time. Assume that we want to proceed with discretization of second general transition. Let the set $\mathcal{S}_{2}^{D}=\left\{s_{2}^{(1)}, \cdots s_{2}^{(n)}\right\}$ be a discretization of the support of $S_{2}$ denoted by $\mathcal{S}_{2}$, as before. Moreover, let $P_{S_{2}}$ be a probability measure defined on $\mathcal{S}_{2}^{D}$. Then the probability that an STL formula $\Psi$ holds at time $\tau$ can be computed using the law of total probability, as:

$$
\operatorname{Prob}^{\tau}(\Psi)=\sum_{s_{2}^{(i)} \in \mathcal{S}_{2}^{D}} \operatorname{Prob}^{\tau}\left(\Psi \mid S_{2}=s_{2}^{(i)}\right) P_{S_{2}}\left(S_{2}=s_{2}^{(i)}\right),
$$

where $\operatorname{Prob}^{\tau}\left(\Psi \mid S_{2}=s_{2}^{(i)}\right)$ is the probability that $\Psi$ is satisfied at time $\tau$, conditioned on the second general transition to fire at time $s_{2}^{(i)}$.

Now assume we have $n$ stochastic variables, and let $\mathcal{S}_{j}^{D}$ be a discretization of support of $j$ th random variable for $2 \leq j \leq n$. By a recursive application of the above equation we have:

$$
\begin{aligned}
& \operatorname{Prob}^{\tau}(\Psi)= \\
& \sum_{s_{n}^{(i)} \in \mathcal{S}_{n}^{D}} \cdots \sum_{s_{2}^{(i)} \in \mathcal{S}_{2}^{D}}\left(\operatorname{Prob}^{\tau}\left(\Psi \mid S_{n}=s_{n}^{(i)}, \cdots, S_{2}=s_{2}^{(i)}\right) \prod_{j=2}^{n} P_{S_{j}}\left(S_{j}=s_{j}^{(i)}\right)\right) .
\end{aligned}
$$

For simplicity, for the rest of this section, we assume only two stochastic variables.

If the second transition follows a discrete probability distribution with bounded support, then we can define $\mathcal{S}_{2}^{D}=\mathcal{S}_{2}$. Hence it is possible to compute $\operatorname{Prob}^{\tau}(\Psi)$ exactly, since the probability measure $P_{S_{2}}\left(S_{2}=s_{i}\right)$ is 
already defined. However, if the support of the firing time is unbounded, the summation has to be truncated for a certain value $s_{2}^{\max } \in \mathcal{S}_{2}^{D}$. In case of a continuous probability distribution, we need to construct $\mathcal{S}_{2}^{D}$ by discretizing the support of the firing times of the second general transition. Moreover, we define the probability measure $P_{S_{2}}\left(S_{2}=s_{2}^{(i)}\right)$ as the total mass of probability between two consecutive discretization points:

$$
P_{S_{2}}\left(s_{2}^{(i)}\right)=G_{2}\left(s_{2}^{(i+1)}\right)-G_{2}\left(s_{2}^{(i)}\right)=\int_{s_{2}^{(i)}}^{s_{2}^{(i+1)}} g_{2}(s) d s
$$

in which, as before, $g_{2}$ is the continuous probability distribution of the second general transition. Both truncation and discretization clearly result in an approximate computation of the desired probability.

The discretization, i,e., construction of the set $\mathcal{S}_{2}^{D}$, can be done in two different ways, namely using a predefined discretization step, or using the Monte Carlo method [94]. For the former we use discretization step $\Delta s_{2}$ to build the set $\mathcal{S}_{2}^{D}$ :

$$
\mathcal{S}_{2}^{D}=\left\{s_{2}^{(i)}=a+i . \Delta s_{2}\right\}_{i=0}^{i=m},
$$

where, $a$ is the starting point, and $m$ is the total number of discretization points. In contrast, in the Monte Carlo method, we choose the discretization points based on the probability distribution of $S_{2}$, i.e., those points with higher probability are more likely to be chosen. This is indeed more reasonable as we are choosing points which are likely to contribute more probability to the final probability. We will investigate and compare the effect of these two methods in detail, in Chapter 8 (Section 8.3.2) for a real world application.

Algorithm 7.1 shows the process of computing the probability given by Equation (7.1) for the case of two general transitions. As input it receives property $\Psi$, time point $\tau$ for which we want to know whether the property holds, and the discretized support $\mathcal{S}_{2}^{D}$. Through lines 24 , we iterate over all possible values of firing times of the second general transition, $s_{2}$, and generate an STD for each value of $s_{2}$. Function generateSTD $\left(s_{2}\right)$, describes the process of generating an STD for which it is assumed that the second general transition is going to fire at time $s_{2}$. Particularly, the second general transition, for this case, can be replaced by a deterministic transition with firing time at $s_{2}$. In line 4 , the probability of $\Psi$ holding at time $\tau$ for the generated STD, is computed through function $\operatorname{Prob}^{\tau}(s t d, \Psi)$. Finally, this probability is added to the final probability with weight $P_{S_{2}}\left(S_{2}=s_{2}\right)$, according to (7.1).

By using this approximate solution we clearly lose the advantage of computing exact probabilities; however, we are able to overcome the restriction to one general transition for the analysis of HPnGs. In Chapter 8 (Section 8.3), we will illustrate how this method can effectively be 
Algorithm 7.1 ComputeProb $\left(\Psi, \tau, \mathcal{S}_{2}^{D}\right)$

Require: Property $\Psi$, investigation time $\tau$, and discretized support of the second second stochastic variable, $\mathcal{S}_{2}^{D}$.

Ensure: Probability of holding formula $\Psi$

1: $\pi^{\tau} \leftarrow 0$

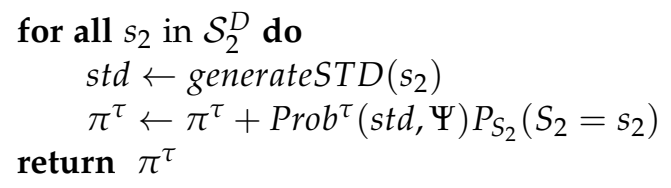

used for the analysis of an HPnG model of a real world application with two general transitions.

\subsubsection{Control Example}

In order to illustrate the discretization idea, in this section we reconsider the control example from Section 3.3.2, which is presented again in Figure 7.1 for convenience. Suppose, we want to check whether even after the failure (transition $T_{f}$ firing) of the producer pump (transition $T_{p}$ ) the fluid level in the reservoir (place $P_{m}$ ) always stays between one and ten. For this purpose we model check the following STL formula at the starting time 0 :

$$
\Phi=t t \mathcal{U}^{\left[\alpha, \alpha+t_{\max }\right]}\left(x_{P_{m}} \leq 1 \vee x_{P_{m}} \geq 10\right)
$$

In this formula variable $x_{P_{m}}$ represents the amount of fluid in the reservoir $P_{m}, \alpha$ is the time of occurrence of the failure at the producer pump $\left(T_{p}\right)$, and $t_{\max }$ is the maximum time of analysis, within which we want to check that a state with less than 1 amount of fluid or more than 10 amounts of fluid is only reached with a very small probability. We investigate this STL formula for the case of $\alpha=3$.

For this example we discretize the the firing time of the general transition $T_{a f}$, and for each possible realization of its firing time we compute a new STD. This is illustrated in Figure 7.2 which shows four different STDs for different firing times of the general transition $T_{a f}$, namely for a realization of the failure at $0.5,1.5,2.5$ and 3.5 minutes after the demand has been turned on. Note that we start the analysis with 6 litres of water in the tank.

Regions in which property $\left(x_{P_{m}} \leq 1 \vee x_{P_{m}} \geq 10\right)$ does not hold and that are within the time interval $\left[\alpha, \alpha+t_{\max }\right]$ are outlined in green. Moreover, red regions indicate that the formula $\left(x_{P_{m}} \leq 1 \vee x_{P_{m}} \geq 10\right)$ holds, before reaching the maximum time, $t_{\max }=10$. It is not desirable to be in a red region before the maximum time $t_{\max }=10$, because in such 


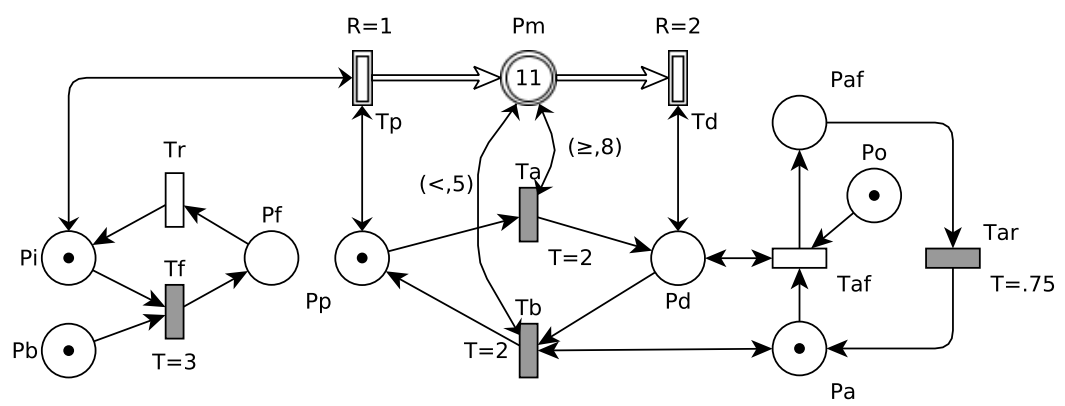

Figure 7.1.: A simple control example. The amount of fluid in the reservoir $P_{m}$ is supposed to remain between 1 and 10 .

regions the control condition is violated. Hence, in practice one would check whether formula $\Phi$ holds with a very small probability.

When the actuator $T_{b}$ fails 0.5 minutes after the demand has been turned on, i.e., the actuator $T_{a}$ has fired, and $P_{m}$ is now decreasing at rate 2, the amount of water in the tank at the time of the failure is 9 litres and since the repair only takes 0.75 minutes, the failure will be repaired when the amount of water in the tank is 7.5 litres $(9-2 \times 0.75)$. Since this is still more than 5 litres which is the condition for switching off the demand and the input back on, the failure has no impact on the system, hence, the probability that $\Phi$ holds, given a failure of $T_{b}$ after 0.5 minutes, is zero. This can be seen in the corresponding STD, as shown in Figure 7.2a, as it is not possible to reach a red region in the described time interval. Note that this scenario is independent of the other failure that switches off the input directly in three minutes after the start of analysis.

However, the impact of a failure that occurs later, i.e., 1.5 minutes after the demand has been turned on, depends on how quickly the failure at the input is repaired (modelled by general transition $T_{r}$ ). In case the failure at the input has not been repaired the amount of water in the tank is 6 litres at the moment of the failure at $T_{b}$. During the repair of $T_{b}$ the amount of water further decreases to 4.5 litres $(6-2 \times 0.75)$ and when $T_{b}$ becomes enabled and fires after 2 minutes the amount of water has dropped to 0.5 liter $(4.5-2 \times 2.0)$, which violates the control condition, hence property $\Phi$ holds. However, in case the failure at the input is repaired quickly, the amount of water in the tank is larger at the moment $T_{b}$ fails and this prevents a violation of the control condition. The corresponding STD for failure of the actuator at 1.5 minutes is shown in Figure 7.2b. One can observe that for small values of the repair time at the input (firing time of $T_{r}$ ), the system reaches the maximum 


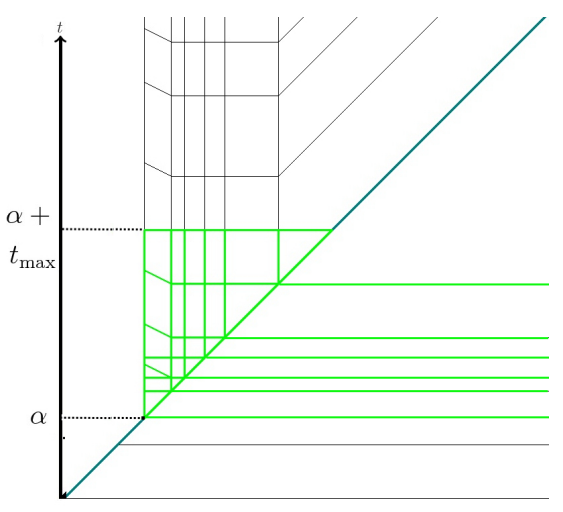

(a) Failure at 0.5 minute.

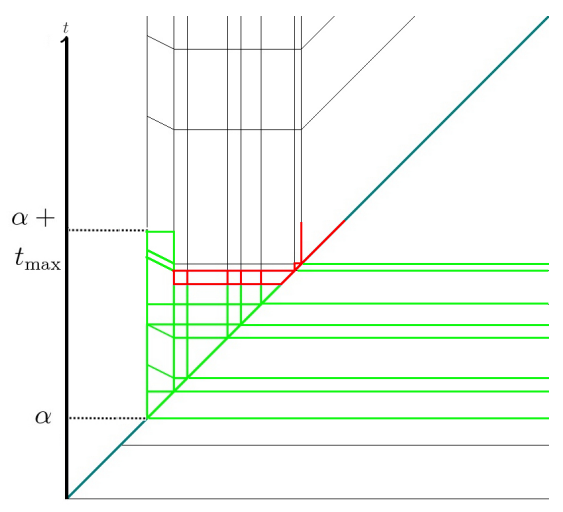

(c) Failure at 2.5 minutes.

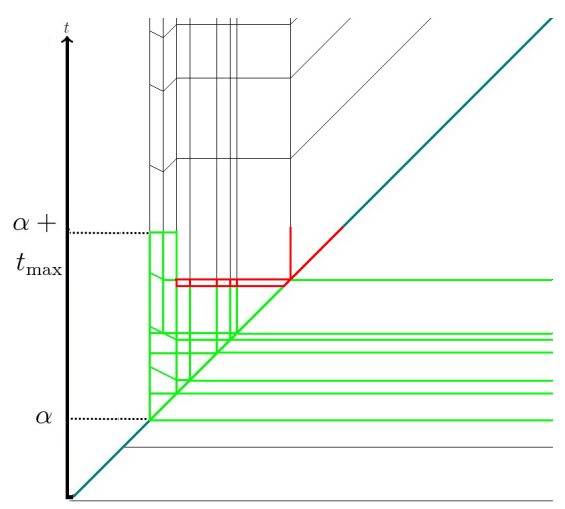

(b) Failure at 1.5 minute.

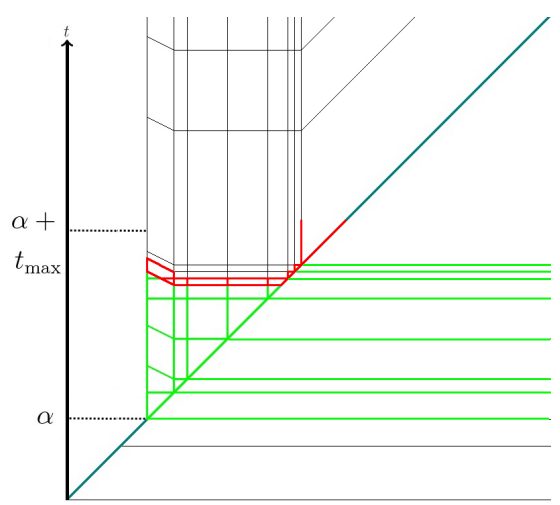

(d) Failure at 3.5 minutes.

Figure 7.2.: Four instances of STD for different firing times of the second general transition $T_{a f}$. Green and red regions domonstrate desirable and undesirable regions according to Formula 7.4, respectively. 
time without hitting red regions, while for larger values does hit the red regions.

A similar reasoning holds for a failure 2.5 minutes after the demand has been turned on. As shown in Figure 7.2C, again, the right part of the system evolutions, representing a late repair of the input, ends in a red region in the specified time interval.

A failure that takes place 3.5 minutes after the demand is switched on inevitably leads to a violation of the control condition, hence $\Phi$ will hold with probability 1 . This means that the system will always violate the control condition no matter how fast the failure at the input is repaired. This is due to the fact that the failure at actuator $T_{b}$ happens when there is very little water in the tank and the input should be switched on quickly. However, due to the repair delay of 0.75 minutes, we surely have less than 1 litre water in the tank, once $T_{b}$ fires. This can be seen in Figure $7.2 \mathrm{~d}$, as there is no possibility of reaching the maximum time, $t_{\max }+\alpha$, without passing a red region.

The shown STDs, illustrate the idea of discretizing the firing times of the second general transition. Once all the STDs for all possible firing times are obtained, the probability that a given formula $\Phi$ holds can be computed according to Equation (7.1), as described in Algorithm 7.1. The feasibility of the overall idea for more complex cases will be demonstrated in Section 8.3, accompanied with more theoretical analysis. Moreover, over there, we discuss and compare different discretization methods, namely fixed steps and Monte Carlo method. We postpone this because the effect of these two methods can be seen more clearly for a larger example.

\subsection{PROBABILISTIC TIME TRANSITIONS}

In this section, we confine the model to the case that the associated probability distribution of all discrete transitions have discrete and finite support. We call transitions of this sort Probabilistic Timed Transitions (PTTs). This is because PTTs fire probabilistically at a given finite realvalued set. This finiteness for values of stochastic variables, allows us to undertake a completely different approach than the one we have so far investigated.

In this new setting, like before, one state of the system can have several successor states, which are chosen according to the probability distribution of the respective PTT. But now the number of these successors states are by assumption finite, hence, no grouping (like the concept of regions) is needed to overcome the infiniteness. As a result, the state evolution over time resembles a tree with a finite branching factor. To investigate whether a certain system property holds with a certain probability at a given time, we can generate the state evolution tree up to that time, 
and accumulate the probability that the given property holds among the leaves of the generated tree.

Unfortunately, since the number of successors of each state equals the total number of possible firing times of the enabled PTTs, the state evolution tree grows exponentially. Hence, in the presence of many of such probabilistic choices, it is often infeasible to investigate the full state evolution tree (even if only up to a certain time) and to compute the exact probability for a given property. Instead, we propose to compute both, an under- and over-approximation of the probability that a given property holds and refine these approximations by iteratively taking a part of the state evolution tree into account, until we can decide whether the probability that the property of interest holds exceeds a certain threshold.

Clearly, it is important to use efficient search algorithms to decide, iteratively, which parts of the state evolution tree to explore. For this, we assign scores to nodes (to be difined in Section 7.2.2) of the state evolution tree, based on current knowledge, i.e., how much probability they attribute, and based on a prediction for the future, i.e., an estimation based on the property of interest. Then, we use an instance of a bestfirst search algorithm to expand nodes with a high score early in the computation [95].

The remainder of this section is organized as follows. In Section 7.2.1 we introduce needed notations and definitions. Section 7.2.2 discusses the generation process of the state evolution tree, and Section 7.2.3 investigates how to efficiently explore and expand it to check system properties. Finally, in Section 7.2.4 we provide a case study to show the feasibility of the approach.

\subsubsection{Notation and definitions}

In this section in order to prevent confusion among different indexing we use lower case letters to refer to PTTs. Each PTT is defined by its potential firing times and their corresponding probabilities. Recall from Section 3.1 that each discrete transition was being assigned a CDF via mapping $\phi_{G}^{\mathcal{T}}$. As mentioned before PTTs are special type of discrete transitions with finite support for their firing times. More precisely, a PTT $a$ through mapping $\phi_{G}^{\mathcal{T}}$ is assigned to a CDF $G_{a}: \mathcal{S}_{a} \rightarrow[0,1]$, where $\mathcal{S}_{a}=\left\{t_{1}^{a}, \ldots, t_{m_{a}}^{a}\right\}$, is a finite set of real numbers, and $m_{a}$ is the number of possible firing times for $a$. Each $t_{i}^{a}$ specifies a possible firing time for PTT $a$, which is associated with a probability $\alpha_{i}^{a} \in[0,1]$. Therefore we have: $G_{a}\left(t_{i}^{a}\right)=\sum_{j=1}^{i} \alpha_{j}^{a}$. Note that we have $0 \leq t_{1}<t_{2}<\cdots<t_{m_{a}}$, and $\sum_{i=1}^{m_{a}} \alpha_{i}^{a}=1$. Moreover, the possibility that the PTT $a$ never fires can be be included by assigning $t_{m_{a}}^{a}$ to infinity. Alternatively, we may 
characterize a PTT by the sequence $\mu=\left(t_{1}^{a}: \alpha_{1}^{a}, t_{2}^{a}: \alpha_{2}^{a}, \ldots, t_{m_{a}}^{a}: \alpha_{m_{a}}^{a}\right)$. We call $\mu$ the firing density of the PTT.

In this section for conflict resolution among PTTs we use probabilistic selections, as introduced in Section 3.4. This means that whenever there is a conflict between a set of PTTs, we make a selection among them based on Equation (3.3). This means that their firing probabilities are modified. We will discuss this in following.

\subsubsection{State evolution tree}

In the approach using STDs, the value of stochastic variable(s) characterizing the firing time of general transitions was chosen from a dense set and the state space was generated by partitioning and forming regions with similar properties, over the values of these dense sets. The advantage of using PTTs is that the firing times are chosen from discrete sets. Hence, going from one state to another can be interpreted as a probabilistic jump. In this section, we analyse the state evolution of the system over time, and provide a method based on effective search algorithms for generating and exploring the state evolution.

Like before in each state of the system, three types of potential events can occur: (i) a continuous place reaches its lower or upper boundary, (ii) a continuous place reaches the weight of the guard arc connected to it, and (iii) an enabled discrete transition fires. Among these, the third type, since we are only dealing with PTTs now, is a probabilistic choice from a finite set of events. Therefore the next state is determined probabilistically, i.e., each state may have several successors, each of them chosen with a certain probability. This suggests that the state evolution of the system over time forms a directed tree structure where each node represents a state and each edge an event leading from the current state to the next state of the system. Each node embodies the state $\Gamma$ of the system which is the same as the system state introduced in Chapter 3, together with the time at which the system does enter that state. Moreover, each edge is associated with the probability of occurrence of the corresponding event. For each node $u$, we denote its state and time of entry by $\Gamma(u)$ and $t(u)$, respectively.

Figure 7.3 shows a schematic state evolution of the system, where $u_{0}$ is the root of the tree, i.e., the node with the initial state $\Gamma_{0}$, at time $t=0$, i.e., $\Gamma\left(u_{0}\right)=\Gamma_{0}$ and $t\left(u_{0}\right)=0$. For each node $u_{i}$ the probability of going from that node to node $u_{j}$ is denoted by $p\left(u_{i} \rightarrow u_{j}\right)$. This is the probability associated with the event that takes the system from $\Gamma\left(u_{i}\right)$ to $\Gamma\left(u_{j}\right)$.

As stated earlier, event types (i) and (ii) are deterministic and occur with probability 1 . Hence, a node for which the next occurring event is deterministic has only one successor. For event type (iii), instead, 


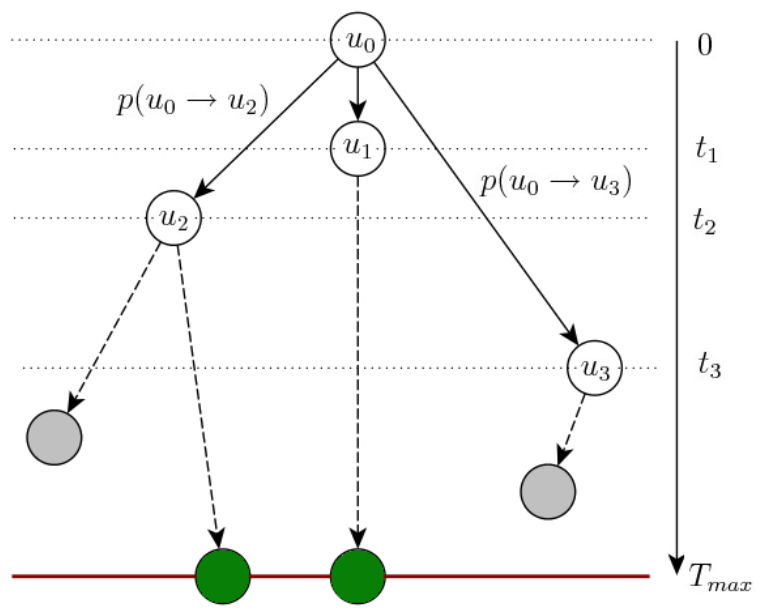

Figure 7.3.: A state evolution tree w.r.t. a maximum time $T_{\max }$.

we have several successors, each of which occurs according to the probability of the corresponding event. To resolve deterministic events, we iterate over all continuous places and check whether they can reach their upper or lower limits, type (i), or the weight of a corresponding guard arc, type (ii), before occurrence of an event of type (iii). In the following, we focus on probabilistic events.

With each node, we associate the time when the system entered the corresponding state. The occurrence of each event advances the system in time. Figure 7.3 shows the evolution of time vertically, each horizontal dashed line depicts the time associated with the node(s) on it.

We now calculate the probability of moving from one node to another by firing a PTT. Assume that, at node $u$, the set $\mathcal{T}_{E}^{D}(u)$ contains all enabled transitions, and, for each $a \in \mathcal{T}_{E}^{D}(u)$, let $\left(t_{1}^{a}: \alpha_{1}^{a}, \cdots, t_{k}^{a}: \alpha_{k}^{a}\right.$, $\left.\cdots, t_{m_{a}}^{a}: \alpha_{m_{a}}^{a}\right)$ be the associated firing density. Let $m_{a}(t)$ be the index of the last time point at which transition $a$ may fire before time $t$, i.e., $m_{a}(t)=\max \left\{1 \leq k \leq m_{a}: t_{k}^{a}<t\right\}$. Then the probability that an arbitrary $b \in \mathcal{T}_{E}^{D}(u)$ fires at time $t_{i}^{b}$ and takes the system to node $u_{i}^{b}$ equals the probability that none of the enabled transitions fires before time $t_{i}^{b}$ and that transition $b$ fires at time $t_{i}^{b}$ (with probability $\alpha_{i}^{b}$ ). To compute the probability that no transition fires before time $t_{i}^{b}$, we need to multiply all the complementary probabilities of firing of all the enabled transitions. Hence we have:

$$
p\left(u \rightarrow u_{i}^{b}\right)=\alpha_{i}^{b} \cdot \prod_{a \in \mathcal{T}_{E}^{D}(u)-\{b\}}\left(1-\sum_{k=1}^{m_{a}\left(t_{i}^{b}\right)} \alpha_{k}^{a}\right)
$$


The product considers all enabled transitions $a \in \mathcal{T}_{E}^{D}(u)$, except $b$ itself, and computes the probability that none of these fire before time $t_{i}^{b}$.

As mentioned earlier, if several transitions can fire at the same time, we use probabilistic selection to resolve conflicts. Therefore, the computed probability in the above equation is distributed among the conflicting PTTs, with the strategy given in Equation (3.3). Hence the jump probabilities are modified as follows:

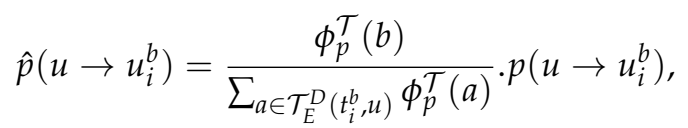

where, $\mathcal{T}_{E}^{D}\left(t_{i}^{b}, u\right)$ is the set of transition that can fire at the same time as $b$, i.e., at $t_{i}^{b}$.

Now, let node $u$ be reachable from $u_{0}$ along path $u_{0} \rightsquigarrow u$. Then, since the path between each two nodes is a unique, the probability of being in $u, p(u)$, is given by the product of the probabilities of all the edges on the path:

$$
p(u)=\prod_{\left(u_{i}, u_{i+1}\right) \in u_{0} \rightsquigarrow u} \hat{p}\left(u_{i} \rightarrow u_{i+1}\right) .
$$

Algorithm 7.2 shows the procedure to expand a given node $u$, i.e., generate the set of its successors $\mathcal{N}_{u}$, until a maximum analysis time $T_{\max }$. Lines 1-2 initialize the final set $\mathcal{N}_{u}$ to the empty set and the time to the next event $\Delta t_{n e x t}$ to the distance to the maximum analysis time. Lines 3-4 iterate over all continuous places to find deterministic events of type (i) and (ii). The next event of one of these types is the one that can occur first, at time $\Delta t_{\text {next }}$. Note that $\Delta t_{P}$, the time to the event associated with place $P$, can be computed based on the drift of $P$ and its current fluid content by solving a simple linear equation. Then, we iterate over all enabled PTTs (line 6), and for each one we consider those firing times that can occur before $\Delta t_{\text {next }}$, the time of occurrence of the next deterministic event (line 7). As discussed earlier, each possible firing time of a PTT introduces a new successor to $u$. For each transition $a$ and its possible firing time $t_{i}^{a}$, lines 8-9 create a node $u_{i}^{a}$ and update its state and entry time based on the state and the entry time of $u$ (as given in Algorithm 5.5, Chapter 5). Line 10 computes the probability of being in $u_{i}^{a}$ based on Equations (7.5), (7.6) and (7.7). Finally, the new node is added to $\mathcal{N}_{u}$, the set of successors of $u$.

As mentioned earlier, it is possible that none of the PTTs fires before time $\Delta t_{\text {next }}$, although given the chance. In this case the next deterministic event occurs at the previously computed time $\Delta t_{\text {next }}$. If no deterministic event can occur before time $T_{\max }$, a special node $u_{\max }$ is created, which corresponds to reaching the maximum time horizon. This is done in lines $13-16$, and probability $1-p_{\text {total }}$ (where $p_{\text {total }}$ is the total probability of firing of PTTs) is assigned to the edge leading to $u_{\max }$. Note the 


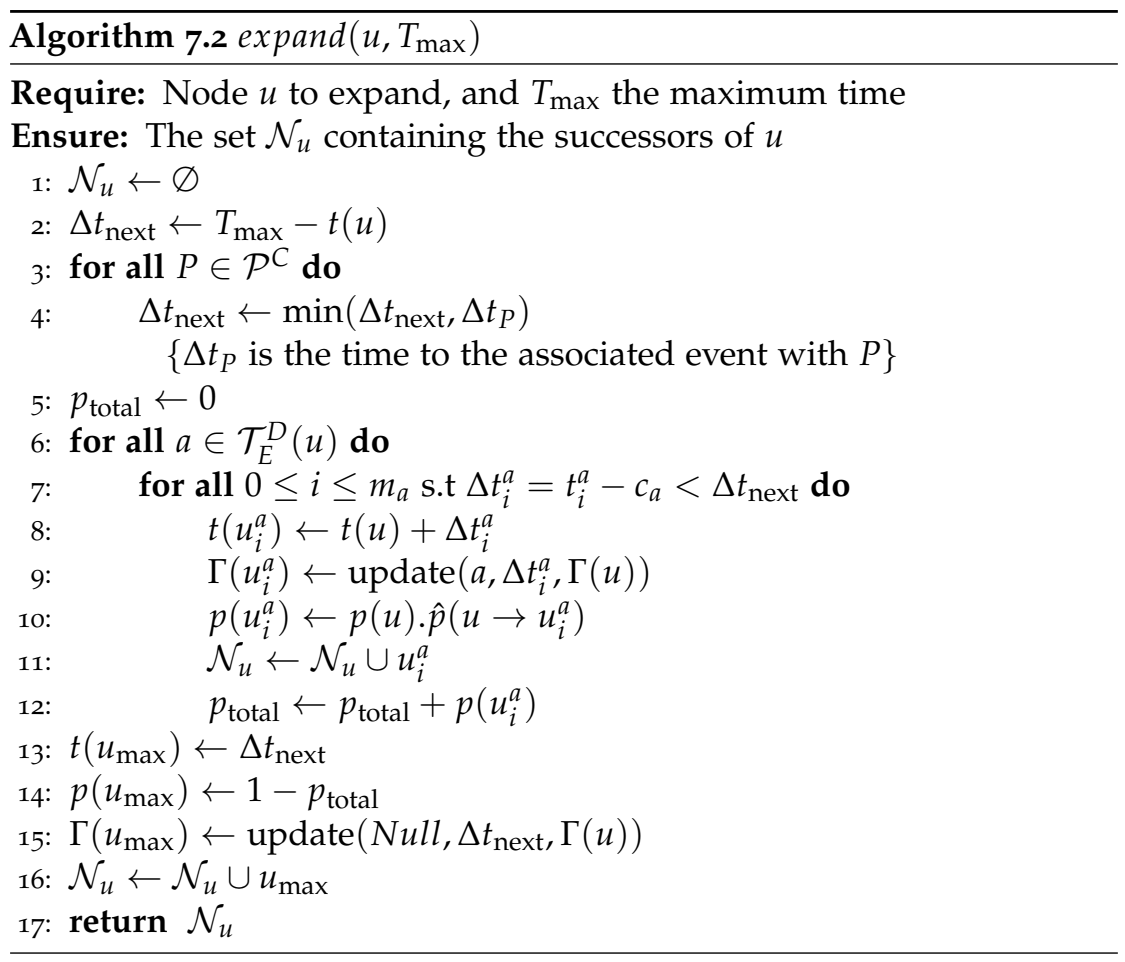

first argument to the function update, in line 15, indicating the firing transition, is set as Null, since no transition is fired to enter $u_{\max }$.

Note that $T_{\max }$ determines the depth of the state evolution tree, hence influences its shape since, for each node, only those events that can happen before $T_{\max }$ are going to be considered. In Figure $7 \cdot 3$, for instance, if $T_{\max }$ is less than $t_{3}$, then node $u_{3}$ will not be generated and is replaced by $u_{\text {max }}$. Hence, the leaves of the tree are going to be those nodes corresponding to reaching the maximum time, except for the case that an event occurs exactly at time $T_{\max }$.

Finally we note that like before, in order to avoid Zeno behaviour (Section 2.2.2), we require the models to be bounded (Definition 2.3). Hence, infinite sequences of vanishing markings can be detected and removed [67-69]. This ensures that we always reach a tangible marking in a finite number of steps. In other words, the number of nodes in the state evolution tree with a finite time bound is always finite; therefore, for a bounded model and a finite time bound, the algorithm always terminates. 


\subsubsection{Exploring the state evolution}

In the following we discusses the generation and exploration of the state evolution tree in an effective way with respect to the given measure of interest. In this section for simplicity we only consider state-based formulas, i.e., the Until operator is excluded:

$$
\Psi:=\mathrm{tt}\left|x_{P} \geq c\right| m_{P}=a|\neg \Psi| \Psi \wedge \Psi .
$$

Investigating Until formulas, as in Section 6.2, needs moving ahead in time, hence is involved with more technicalities, which we like to avoid in this section.

Let $\mathcal{U}^{\tau}$ be the set of all nodes the system can be in at time $\tau$. Hence, the probability of formula $\Psi$ being satisfied at time $\tau$, can be written as:

$$
\pi^{\Psi}(\tau)=\sum_{u \in \mathcal{U}^{\tau}} \mathbb{1}_{\Psi}(u) p(u)=\sum_{u \in \mathcal{U}^{\tau}} \pi_{u}^{\Psi}(\tau),
$$

where $\mathbb{1}_{\Psi}(u)$ is the indicator function, which equals one if $\Psi$ holds for $u$ and zero otherwise. Moreover, we use $\pi_{u}^{\Psi}(\tau)=\mathbb{1}_{\Psi}(u) p(u)$ to show the amount of probability contributed by node $u$ to the property $\Psi$ at time $\tau$.

Note that the above equation is the analogous version of Equations (4.1) and (6.2). However, since here the support of stochastic variables is discrete and finite, instead of being a dense set, the integration is replaced by a countable summation over set of states the system can be in, at a given time $\tau$.

The exact computation of $\pi^{\Psi}(\tau)$ is only possible if we determine the set $\mathcal{U}^{\tau}$, which in turn is only possible if we explore the entire state evolution tree. In the following we show how we can instead approximate its value by visiting only a portion of the state evolution tree.

\section{Exploration}

The problem of finding the possible states of the system at a given time $\tau$ can be reduced to a tree search problem where the goal nodes are those where the system can reside in at time $\tau$. In our framework, this is equivalent to generating the state evolution until $T_{\max }=\tau$. Consequently, $\mathcal{U}^{\tau}$, in (7.8) contains leaves of the generated state evolution tree. Figure $7 \cdot 3$ depicts these goal nodes in green.

Unfortunately, the state evolution tree grows exponentially in the number of PTTs and their corresponding number of firings. More precisely, the branching factor at node $u$ is given by:

$$
b_{u}=\left(\sum_{a \in \mathcal{T}_{E}^{D}(u)} m_{a}\right)+1 \leq\left(\sum_{a \in \mathcal{T}^{D}} m_{a}\right)+1=b_{\text {max }},
$$


i.e., the total number of possible firings of enabled transitions plus one, to include the possibility that no transition fires. The upper bound of this branching factor occurs when all the PTTs are enabled. Let $\delta$ be the minimum time distance between occurrence of two consecutive events. Then, the corresponding tree has at most $\tau / \delta$ "levels". Hence, the total number of nodes in the state evolution tree is $O\left(b_{\max }^{\tau / \delta+1}\right)$. Without an effective method for choosing and expanding the nodes, exploring the entire state evolution for a large number of firing times is infeasible.

Since, in practice, because of the state space explosion, only a portion of the state evolution can be traversed, we compute an approximation of the probability that a given formula holds as follows. Denote the accumulated probabilities that formula $\Psi$ and its negation $\neg \Psi$ hold at time $\tau$ by $\hat{\pi}^{\Psi}(\tau)$ and $\hat{\pi}^{\Psi}(\tau)$, respectively. We have $\hat{\pi}^{\Psi}(\tau) \leq \pi^{\Psi}(\tau)$ and $\hat{\pi}^{\neg \Psi}(\tau) \leq \pi^{\neg \Psi}(\tau)$, where the equality holds if we explore the entire state evolution until time $\tau$. Thus, we have $\hat{\pi}^{\Psi}(\tau)+\hat{\pi}^{\neg \Psi}(\tau) \leq 1$. This means that $\hat{\pi}^{\Psi}(\tau)$ and $1-\hat{\pi}^{\neg \Psi}(\tau)$ provide a lower and upper approximation for $\pi^{\Psi}(\tau)$, respectively:

$$
\hat{\pi}^{\Psi}(\tau) \leq \pi^{\Psi}(\tau) \leq 1-\hat{\pi}^{\neg \Psi}(\tau) .
$$

If we seek to investigate whether the probability that $\Psi$ holds at time $\tau$ is at least a given threshold $q$, we can stop the exploration if either $\hat{\pi}^{\Psi}(\tau) \geq q$ or $\hat{\pi}^{\neg \Psi}(\tau)>1-q$. In other words, we cannot stop as long as:

$$
\hat{\pi}^{\Psi}(\tau) \leq q<1-\hat{\pi}^{\neg \Psi}(\tau) .
$$

Algorithm 7.3 shows the generic procedure for traversing the state evolution tree. It starts with the root of the tree, $u_{0}$, i.e., the node with the initial state of the system, property $\Psi$, time of interest $\tau$, and the probability threshold $q$. During the exploration process we try to expand the most promising node at each time, hence we try to use a best-first search policy. For this, we assign to each node a score indicating its importance, and expand nodes in the order of their scores. As we will address later, this value can depend on the probability of being in that node, its associated state, or the formula under investigation. We keep the nodes that have been generated but not yet expanded in set $Q$, stored as a sorted data structure based on the node scores, e.g., a priority queue. At the beginning, both $\hat{\pi}^{\Psi}(\tau)$ and $\hat{\pi}^{\Psi}(\tau)$ are initialized to zero since no node has been visited yet, and $Q$ contains only $u_{0}$ (lines 1-2). While $Q$ is not empty or the threshold conditions for $\Psi$ holding are not met, the algorithm continues (line 3). In line 4, the node with the highest score is chosen by calling Q.pop() and expanded in line 5 , based on Algorithm 7.2. If any of the new nodes is a goal node, i.e., its entry time point is equal to $\tau$, it contributes to either $\hat{\pi}^{\Psi}(\tau)$ or $\hat{\pi}^{\Psi}(\tau)$, depending on whether its marking satisfies $\Psi$ or not (lines 7-9). Otherwise, if the new node is not a goal node, it is added to $Q$, so that it can be expanded later (line 11). 


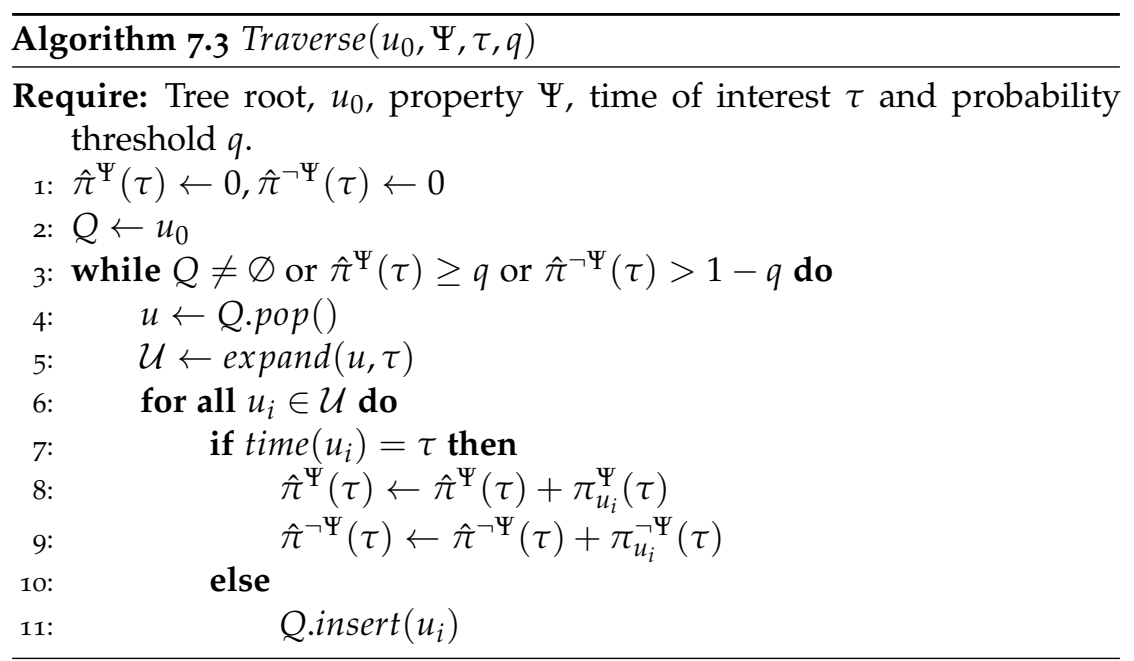

\section{Expansion policies}

As mentioned earlier, we use a best-first search [95] to traverse the state evolution tree, therefore it is important to assign a score to each visited node. This section discusses different scoring policies. In the most general form, as is represented in the $A^{*}$ search method [96], each node $u$ is assigned a score function, $f(u)=g(u)+h(u)$, where $g(u)$ is the actual gain of reaching node $u$ and $h(u)$ is a heuristic function estimating the future gain of reaching a goal node from $u$. Therefore, $f(u)$ is an estimation for the score of reaching a goal node through node $u$.

The algorithm introduced in the previous section accumulates the probability that $\Psi$ holds (or does not hold) at each iteration, and we are interested in reaching a probability threshold as soon as possible. Hence, nodes that contribute more probability mass to either $\hat{\pi}^{\Psi}(\tau)$ or $\hat{\pi}^{\urcorner \Psi}(\tau)$, should be chosen with preference over the others. The most obvious choice would be $g(u)=p(u)$, as given in Equation (7.7), and $h(u)=0$. This, resembles a greedy approach [97], since only our current knowledge of the state evolution tree is used to choose the next node. However, if the probability distribution of nodes follows (or is close to) a uniform distribution this is no different from choosing nodes randomly, as we are not imposing any preference over nodes. On the other hand, choosing a node that leads to a goal node sooner may also be preferable since it reduces the number of intermediate nodes. This implies that nodes with lower distance to the time of interest $t=\tau$ are also advantageous. Moreover, depending on the given formula $\Psi$ and the probability threshold $q$, one may choose $h(u)$ with the goal of steering the search procedure to the portion of the state space that leads to accumulating $\hat{\pi}^{\Psi}(\tau)$ or $\hat{\pi}^{\neg \Psi}(\tau)$ more effectively. 


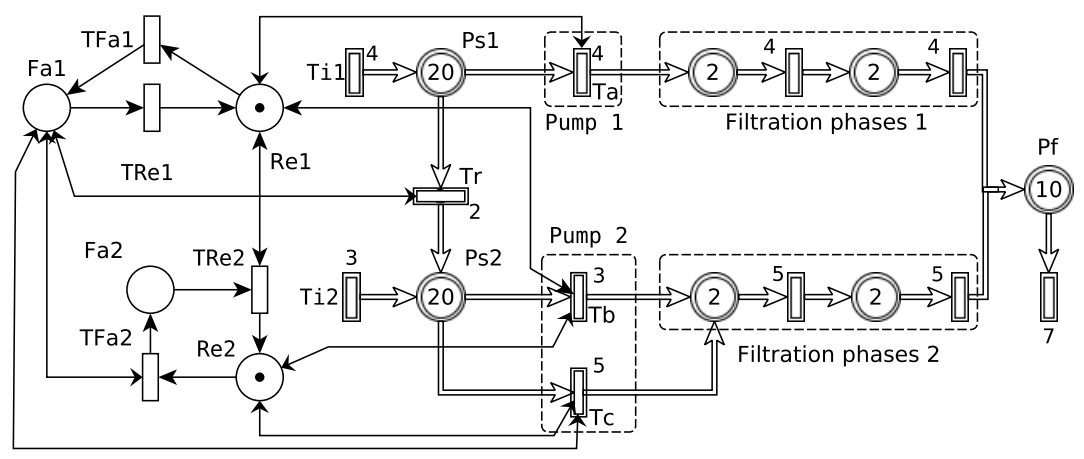

Figure 7.4.: A Petri net model for a water treatment facility with possible cascading failure.

Based on these considerations, one may choose different gain and heuristic functions depending on the structure of the state evolution tree, the probability distribution of nodes, and the given property to investigate. We empirically discuss these ideas further in the next section.

\subsubsection{Case study}

This section shows the feasibility of the proposed approach. Figure $7 \cdot 4$ shows a modified version of the case study from Section 6.1.3, for the case of multiple general transitions. This model consists of two cleaning streets (or filtration phases) providing the content of the final storage, represented by the continuous place $P f$, from which water is distributed to customers with a constant rate of 7 . Each production line starts with a large water softening tank $\left(P_{s_{1}}\right.$ and $\left.P_{S_{2}}\right)$. These two tanks are followed by different filtration phases to remove contaminants from the input water. Each filtration phase is modelled by a continuous transition and place. The total water input to each of these lines is modelled by continuous transitions $T i_{1}$ and $T i_{2}$, with rates 4 and 3 , respectively. Therefore, under normal operation, the total intake of the system matches the total requests.

Continuous transition $\mathrm{Ta}$ models pump 1, responsible for transporting water from the softening tank to the filtration tanks in the first production line. In the second production line, continuous transitions $T b$ and Tc together model pump 2, with the same role as in the first production line. This is because this pump may work with different rates depending on the conditions of the entire system. The pumps connecting the softening tanks to the rest of the phases are the vulnerable points of the system, and the aim of this analysis is to investigate the resilience of the system when there are failures in these pumps. 
PTT $T F a_{1}$ models the probabilistic failure of pump 1 , i.e, transition Ta. If a failure occurs in the first production line, a portion of its load is forwarded to the second production line. This is done through transition $\operatorname{Tr}$ with rate 2, and causes extra load for the second production line. At the time of $T a^{\prime}$ s failure, transition $T b$, which was working with rate 3 , is disabled and instead transition $T c$, with rate 5 , is enabled. This is simulating the fact that pump 2 is working under extra load. This extra load may, however, result in a cascaded failure in pump 2, modelled by PTT $\mathrm{TFa}_{2}$. These two failures are also accompanied by probabilistic repairs, modelled by PTTs $T R e_{1}$ and $T R e_{2}$, respectively.

This model contains four PTTs, which may fire several times, by being enabled and disabled consecutively. Since these PTTs model failure and repair, one may expect that the firing probability distribution is increasing, i.e., the probability of firing grows for larger firing times. To model this we use a linear increase scheme, i.e., we consider a discretized version of a uniform cumulative distribution for their probability density function. In other words, we assign each PTT an interval $\left[0, T_{m}\right]$ and an integer $n$, where $T_{m}$ is the latest time at which the PTT will fire, and $n$ is the number of equally distanced discretization points, i.e., the number of possible firing times.

More formally, if a PTT is supposed to fire at the latest at time $T_{m}$, with $n$ possible firing times, then the difference between each consecutive firing times is $T_{m} / n$. Therefore the probability of a firing at time $t_{i}=i \cdot T_{m} / n$ is $\alpha_{i}=i / n \cdot 2 /(n+1)$, in which $2 /(n+1)$ is a normalization factor to ensure $\alpha_{i}$ 's sum up to one. With this probabilistic distribution, the expected firing time of a PTT is $T_{m}(2 n+1) / 3 n$. Figure $7 \cdot 5$ illustrate this probability distribution for the case of $T_{m}=12$ and $n=12$, i.e., discretization step of one. As one can see the probability of firing increases linearly.

In this model, for PTTs $T F a_{1}, T R e_{1}, T F a_{2}$, and $T R e_{2}$, we assume maximum firing times of $12,6,15$, and 6 , which for large $n$ results in average firing times of, 8, 4, 10, and 4, respectively. With this setting, there will be a failure and repair for sure, at most at the respective maximum times. It is possible to assign each PTT with different probability distributions, but we have considered the same distributions for all PTTs in this model, for simplicity.

During the evolution of the system, at most two PTTs are enabled simultaneously. So, assuming $n$ possible firing times, the maximum branching factor is $b_{\max }=2 n$. Among the four PTTs present in the system, the minimum time difference between two possible firings is $\delta=\min \{12,6,15\} / n=6 / n$. Hence, for the time of interest $\tau$, the total number of nodes in the state evolution tree is at most equal to:

$$
N=(2 n)^{\frac{n \tau}{6}}
$$




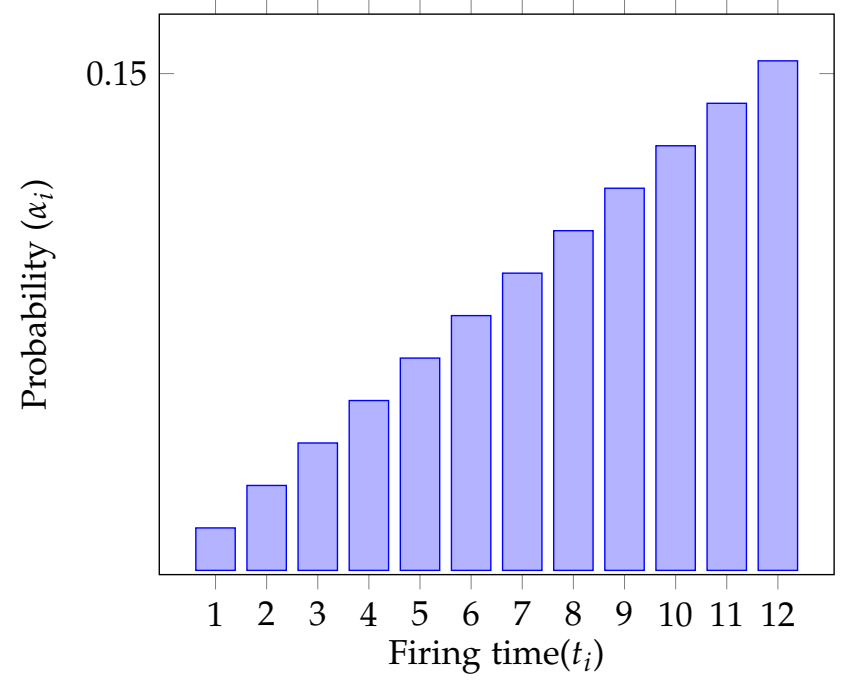

Figure 7.5.: Probability distribution of a PTT for the case of $T_{m}=12$ and $n=12$.

We investigate the probability of not having an empty final storage, that is the probability of not being able to deliver water to the place $P_{f}$ :

$$
\Psi_{1}=x_{P f}>0 .
$$

For the first scenario, as discussed in Section 7.2.3, we favour nodes contributing higher probability, hence we set $f_{1}(u)=p(u)$. We can think of $p(u)$ as the total information we have so far, i.e., $g(u)$, which is our gain by choosing $u$. Hence, this score function implies that the heuristic function $h_{1}(u)$ is zero. Table 7.1 shows the scalability of the approach for different values of $n$, the number of firing times of the PTTs. The estimation of the state space size is computed based on (7.12), for the time of interest $\tau=24$. The unassigned probability is defined as $\pi_{u n}(24)=1-\left(\hat{\pi}^{\Psi_{1}}(24)+\hat{\pi}^{\neg \Psi_{1}}(24)\right)$; this is the probability that still is available for the portion of state space that we have not seen, therefore, we do not know how it contributes to either $\Psi_{1}$ or $\neg \Psi_{1}$. Moreover, the range providing upper and lower approximation for $\pi^{\Psi_{1}}(24)$ is based on (7.10).

As can be seen in Table 7.1, for $n=30$, by generating around 12 million nodes $\left(10^{-111} \%\right.$ of the total state space), we face a $17 \%$ unassigned. According to inequality (7.11), for the case of $n=30$, we cannot reach a conclusion if the given threshold $q$ satisfies: $0.692 \leq q<0.863$.

Even though the computed range in the last row of Table 7.1 is large, we are able to reach a conclusion, especially when checking for thresholds close to zero or one. This is often the case for the verification of safety properties, which either express to have a very low probability of 
Table 7.1.: Approximated probability of $\Psi_{1}$ holding at time $\tau=24$, for different values of $n$ (rounded to 3 decimal places).

\begin{tabular}{r|c|c|c|c|c|c}
$n$ & $N(7.12)$ & \# seen nodes & $\hat{\pi}^{\Psi_{1}}(24)$ & $\hat{\pi}^{\neg \Psi_{1}}(24)$ & $\pi_{u n}(24)$ & $\pi^{\Psi_{1}}(24)$ range \\
\hline 5 & $10^{20}$ & $1.5 \times 10^{5}$ & 0.841 & 0.158 & $10^{-5}$ & {$[0.841,0.842]$} \\
10 & $10^{40}$ & $7.0 \times 10^{6}$ & 0.809 & 0.189 & $10^{-3}$ & {$[0.809,0.812]$} \\
30 & $10^{120}$ & $1.2 \times 10^{7}$ & 0.692 & 0.137 & 0.170 & {$[0.692,0.863]$}
\end{tabular}

service failure, or a high probability of recovery from a failure. Indeed, the proposed algorithm is aimed toward this case. More specifically, the final range is "pushed away" from o and 1, by accumulating both the probability of the given property and of its negation.

Next, we examine the effectiveness of the heuristics. As mentioned in the previous section, this can be done by considering the structure of the model and the given property. We choose a heuristic which favours nodes more likely to lead to a state with fewer failed pumps. For this, we can count the number operational pumps, so we propose $h_{2}(u)=p(u) \cdot\left(n_{R e_{1}}+n_{R e_{2}}\right) / 2$. This means that nodes that contribute higher probability and with less potential for leading to states where the property $\Psi_{1}$ is violated are chosen first. We do not change $g(u)$ from the previous case, so we have $f_{2}(u)=p(u)+h_{2}(u)$.

Figure 7.6 shows the results for the proposed heuristic. The vertical axis represents the number of generated nodes, and the horizontal axis is the probability for different aspects of the property $\Psi_{1}$. The solid and dashed lines are the values of $\hat{\pi}^{\Psi_{1}}(24), 1-\hat{\pi}^{\neg \Psi_{1}}(24)$, and $\pi_{u n}(24)$, for $f_{1}$ and $f_{2}$, i.e., the score functions with or without heuristic functions, respectively. The shaded area depicts the upper and lower approximation of $\pi^{\Psi_{1}}(24)$, which becomes narrower as more nodes are generated. One can see that the heuristic function embodied in $f_{2}$, which guides the process towards nodes with less potential of violating $\Psi_{1}$, results in a slightly faster accumulation of $\hat{\pi}^{\Psi_{1}}(24)$ (green, solid), hence, less unassigned probability, $\pi_{u n}(24)$ (red, solid).

One may notice that in the beginning the probability mass is accumulated faster for both $f_{1}$ and $f_{2}$. This is because, as we first choose nodes contributing high probabilities, after a while the unassigned probability $\pi_{u n}(\tau)$ is distributed among many nodes, each contributing little probability mass. So, the algorithm must traverse many more nodes than at the beginning of the process to accumulate the remaining probability.

\subsection{NOTES ON THE DIFFERENCES OF TWO METHODS}

Both approximation techniques introduced in this chapter, are derived with the idea of confining the support of stochastic variables. However, in the first technique, the method of discretization, one stochastic vari- 

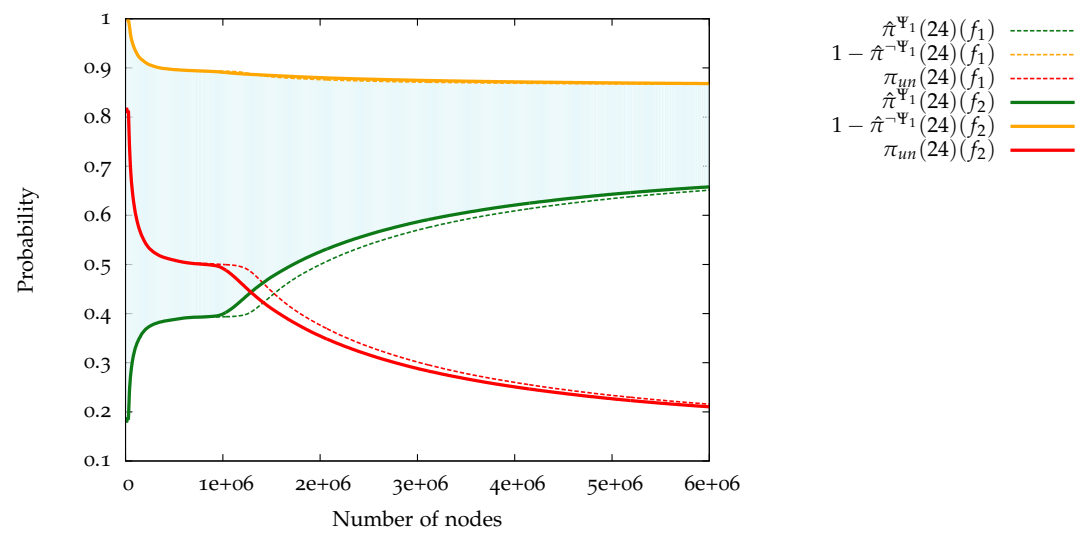

Figure 7.6.: Illustration of performance of different score functions, $f_{1}$ and $f_{2}$ for accumulating $\hat{\pi}^{\Psi_{i}}(24)$ and $\hat{\pi}^{\urcorner \Psi_{i}}(24), i \in\{1,2\}$, for up to 12 million nodes.

able is allowed to have dense support, while in the second, the method of PTTs, all of the stochastic variables are restricted to have discrete supports. This extra restriction in the latter, allowed us to approach the problem in a completely different way. In the following we count the pros and cons of these two methods.

For the method of discretization, generally it is not possible to provide an upper or a lower bound on the error. This is because the formation of STDs depends on many parameters related to structure of the HPnG and its initial state for which the analysis is being performed. However, the method of PTTs, is based on providing lower and upper bounds for the error, which is possible because we choose which part of the state evolution tree is to be generated. This is in contrast with the generation of STDs, which is "blind" to the state of system in each generated region. The provisioning of lower and upper bounds, makes the method of PTTs, suitable for safety analysis, as mentioned before. This is because for safety analysis one is usually interested to know if a certain property is not violated with high (or low) probability. Hence, being able to provide lower and upper approximations for the probability of a measure of interest, we can answer this sort of questions.

The method of PTTs, is scalable with respect to the number of stochastic variables, whereas, the method of discretization is not. This is observable in Equation (7.2), as the complexity of this equation is increasing, as the number of stochastic variables is increased. However, for PTTs, the structure of the state space does not change by increasing the number of stochastic variables. On the other hand, the method of discretization is scalable with respect to the discretization steps, i.e., the size of the discretized support of the stochastic variables. However, as illustrated in 
the case-study of Section 7.2.4 (Table 7.1), the computation power of this method is extremely sensitive to the size of the support of the stochastic variables. This is because the size of the support of stochastic variables determines the branching factor of the state evolution tree. Therefore, if we have a system with several stochastic variables with limited support, the method of PTTs, appears to be favorable. On the other hand, if we have a limited number of stochastic variables, with a large support, e.g., in case of approximation of a dense support, one has to use the method of discretization.

As a final remark we have to mention that, the method of discretization has no limitation on the STL formula, and it includes both statebased and Until formulas. However, the method of PTTs, as is introduced in this thesis, does not include the Until operator. However, we stress that there is no theoretical limitation on this, and future works may overcome this constrain.

\subsection{RELATED WORK}

As mentioned before Hybrid Petri nets form a restricted subclass of hybrid automata where all occurrences of non-determinism are resolved by probabilities [20]. There are several approaches in extending hybrid automata with probabilistic jumps [11,14,88]. One important aspect of our approach is that, although the drifts are restricted to be piecewise deterministic, still it is allowed for discrete and continuous parts of the system to control each other. When adding random behaviour through PTTs, we were able to compute approximate results for this model class. This is often useful since the verification of (probabilistic) hybrid automata has been shown to be undecidable except for some restricted subclasses $[14,17]$.

Abstraction techniques, for obtainining over approximation for probabilities of specific properties, are proposed for the restricted class of probabilistic timed automata [II], and safty verification of probabilistic hybrid automata [88]. This resembles to what we did when analysing hybrid Petri nets with PTTs. The idea of abstraction is based on grouping sets of concrete states into fewer abstract states, and checking safety properties on this abstraction. If unsafe states are unreachable on the abstraction the original system is also safe, and if they are reachable, a refinement of the current abstraction needs to be performed. We have to stress that, the abstraction approach presented in aforementioned works, is quite different from ours, which is based on guided expansion policies.

The idea of choosing firing times of transitions from a finite set is not new. Integer Timed Petri nets [98] are ordinary Petri nets where transition firing times are chosen from a finite integer-valued set. As 
this framework does not include continuous variables, and since state changes take place at integer times, it is possible to use decision diagrams for state representation and reachability analysis. However, the same does not hold for hybrid systems. Moreover, the idea of generating relevant portions of the state space with respect to the measure of interest has also been exploited for analytical solution of large Markov models [99-101]. 
Part III

REAL-WORLD APPLICATIONS 



\section{8}

ENSCHEDE SEWAGE TREATMENT FACILITY

The content of this chapter is based on:

[102] H. Ghasemieh, A. Remke, and B. R. Haverkort. Analysis of a sewage treatment facility using hybrid Petri nets. In 7 th International Conference on Performance Evaluation Methodologies and Tools, pages 165-174. ICST, 2013

[92] H. Ghasemieh, A. Remke, and B. R. Haverkort. Survivability analysis of a sewage treatment facility using hybrid Petri nets. Performance evaluation, 97:36-56, 2016.

Any water that has been affected in quality either by households or by industries is considered waste water. It is usually conveyed in the sewage system of the community to the nearest waste water treatment company. The treatment process consists of several physical, chemical and biological cleaning steps. The goal of the process is to separate the clean water from the so-called sludge, that can later be safely disposed or used as fertilizer. The cleaned water is usually released to surface water in the area.

In the Netherlands, communities normally have contracts with waste water treatment facilities about the maximum amount of waste water that needs to be taken in by the treatment facility. Hence, these facilities are dimensioned to accommodate the treatment of a maximum amount of sewage, often without taking into account the possibility of unforeseen events. However, in the case of very heavy rainfall, which is hard to predict, and actually may occur more often due to climate change, it may happen that the amount of waste water in the community sewage exceeds the available storage capacity. In such cases, the sewage system of the community overflows and waste water is spilled on the streets. Recently, this happened in the city of Enschede, the Netherlands [103, 104] and caused hindrance to citizens and traffic.

Using the modelling tools introduced in this thesis, in this chapter we investigate under which circumstances flooding occurs and what can possibly be done to reduce the probability of such flooding. For this purpose we have employed data like the capacity of tanks and the average 


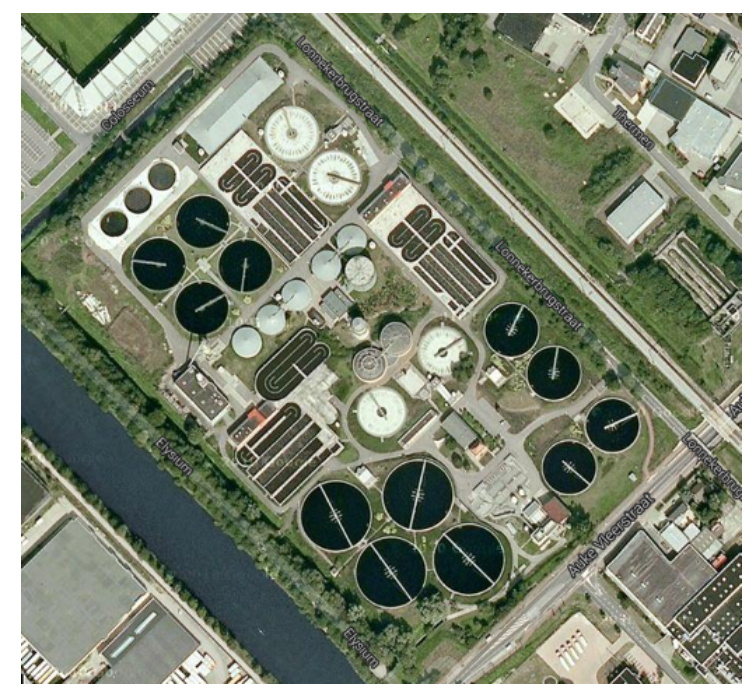

Figure 8.1.: A bird's eye view picture of the sewage treatment facility in Enschede, the Netherlands. The picture is retrieved using Google Maps.

residence time of water in the different cleaning stages from the treatment facility in the city of Enschede. A bird's-eye view of this facility is shown in Figure 8.1.

This chapter is organized as follows. In Section 8.1 we show how the sewage treatment facility can be modelled using the HPnG formalism introduced in this thesis. For this we use the overflow structure provided already in Section 3.3.3. In Section 8.2, we discuss two scenarios for evaluation of the model of sewage treatment facility. Namely, we consider a failure at a vulnerable pump in the network, and a sudden change of weather which affects the intake to the system. In Section 8.3 we use the discretization method introduced in Section 7.1, to combine the two mentioned scenarios. In this section, since we have a concrete example, we investigate the discretization method in details and will provide insight about how it can efficiently be employed. Finally, Section 8.4, discusses the related work.

\subsection{SYSTEM AND MODEL}

This case study models the various stages of the sewage treatment process in an abstract fashion. We are mainly interested in the capacity of each phase and the average amount of time the waste water stays in the different phases. We, however, do not aim at modelling the physical, chemical and biological processes in detail. Then, for a given failure of 
the system at a certain time, we analyse the survivability of the system for changing weather conditions. Fixing the failure to a specific time of the day results in a so-called Given the Occurrence Of Disaster (GOOD) model. Since our evaluation method is so quick, it is easily possible to parametrize the failure time, hence, analyse the system thoroughly.

The main goal of waste water treatment is to separate the input into water that can be safely released into the environment and into thickened sludge which is either used as fertilizer [105] or can be safely disposed [106]. This is done in several stages, where the primary stage mostly involves physical purification, the secondary stage involves chemical and biological treatment, and finally the sludge treatment phase aims at reducing the amount of sludge.

A key component in water treatment facilities is the sedimentation tank. A sedimentation tank physically separates suspended solids from water using gravity [106]. The process is such that the contaminated sewage containing solids enter the tank and stays there, so that solids can sediment at the bottom of the tank, while the water is being removed from top of the tank. In the HPnG formalism as presented before, rate adaptation, as introduced in Section 3.5, prevents both overflow and underflow of a tank, being modelled by a continuous place. However, for sedimentation tanks we need to allow places to overflow. For this we use the overflow structure given in Section 3.3.3.

The HPnG model of the case study is depicted in Figure 8.2; volumes of tanks (continuous places) are indicated in $1000 \mathrm{~m}^{3}$, pump rates (continuous transitions) in $1000 \mathrm{~m}^{3} / h$, and delays (timed transitions) in hours. The capacity of the community sewage system is modelled by an overflow place denoted $P_{c}$, which has input rates that depend on the weather conditions. From this tank the water is pumped into the treatment facility with a maximum rate 12 and in case the input exceeds the capacity of the place and the intake of the treatment facility, the waste water flows into overflow place $P_{o}$ which models the amount of water in the streets. The primary stage of the sewage treatment consists of two phases, namely the sand interceptor and the primary sedimentation tank. The first, as the naming suggests, is responsible for filtering solids like sand from the water. Then the sewage flows in a large tank, which is used to settle the sludge, while the lighter material, like oils, rise on the surface and are removed, and the remaining overflows. In the model the sand interceptor is abstracted through pump $T_{z}$, and the primary sedimentation tank is modelled through overflow place $P_{p s}$.

As mentioned before a sedimentation tank physically separates suspended solids from water using gravity. While the dirt settles at the ground, cleaned water is forwarded to the second cleaning stage. This stage consists of several phases for removing chemical and biological contaminations, modelled by a sequence of continuous transitions and places, before a secondary sedimentation tank separates the biological 


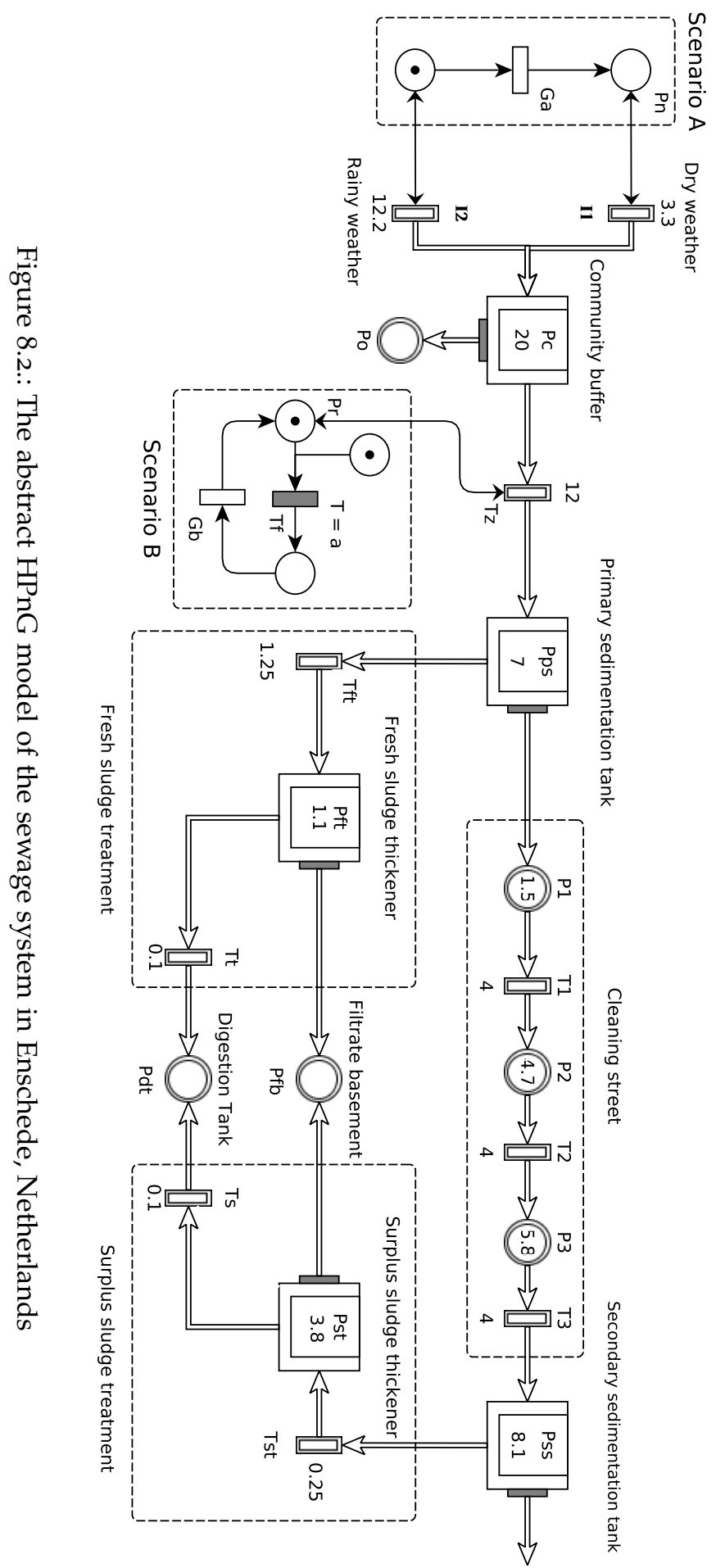


material from the now environmentally friendly sewage water, that can safely be disposed to surface water. The second sedimentation tank is modelled by overflow place $P_{s s}$. The sludge that settles at the primary and secondary sedimentation tank is accumulated and forwarded to the sludge treatment stage. There it is thickened to reduce its volume for easier off-site transport. The sludge from the primary tank is pumped out and forwarded to the fresh sludge thickener. This is also modelled by an overflow place, denoted $P_{f t}$. Sludge is pumped out of the place with a small rate and discharged to the digestion tank which is considered a very large tank. The overflow is directed to the filtrate basement. The same procedure is repeated for the accumulated sludge in the second sedimentation tank.

\subsection{EVALUATIONS}

In the following, we analyse the model in two different ways, namely, by changing the rate of the produced waste water after a random amount of time, and by introducing a stochastic failure at the sand interceptor $T_{z}$, which according to the plant operators is one of the most vulnerable components of the whole process. In Figure 8.2, these two scenarios are depicted by the dashed boxes, labelled as "Scenario A" and "Scenario B". Note that we start the analysis assuming that all tanks in the treatment facility (fluid places and overflow places) are full but the overflow place modelling the community sewage system, $P_{c}$, is empty.

\subsubsection{Scenario A: Heavy rain}

For the first scenario, we assume that the analysis starts with a rainy weather condition, i.e., the production rate of waste water is 12.2, which is slightly more than the capacity of the system, which is 12. Hence, if it continues to rain long enough, the capacity of the community sewage is exceeded and waste water will flood the streets. We assume that the duration of the rain is normally distributed. ${ }^{1}$ This is modelled by the general transition $G_{a}$, which will fire according to the given normal distribution. When $G_{a}$ fires, the production of sewage will switch to normal weather condition, i.e., a production rate of 3.3 .

We would like to analyse how long it may continue to rain without having water in the streets. Using the logic presented in Section 4.2, we

1 This is just an arbitrary assumption. However, the advantage of using HPnGs as modelling formalism is that any arbitrary probability distribution could be used without imposing any additional costs on the analysis. 
want to ensure that the amount of water in the streets is very low until the rain stops, i.e.,

$$
\Phi_{A}=\left(x_{P_{o}}<\epsilon\right) \mathcal{U}^{[0,30]}\left(m_{P_{n}}=1\right),^{2}
$$

where $m_{P_{n}}=1$ means that the rain has stopped and we are back to normal weather conditions. Formula $\Phi_{A}$ is a typical expression of a survivability measure: the first term, before the Until operator is called the safety condition, whereas the one after the Until operator is called the recovery condition. In other words, as defined in Definition 4.3 , for a specific system evolution, $\Phi_{A}$ is satisfied if and only if the safety condition holds until we reach the recovery condition, before the given time bound. We have chosen time bound 30, which is considered to be large enough for this analysis, since it is reasonable to assume that the rain stops within 30 hours.

In the following we investigate the influence of four different parameters, all for varying average duration of rain $\mu$; we consider the capacity of the community buffer $\left(P_{c}\right)$, the rate of the fresh sludge pump $\left(T_{f t}\right)$, the rate of the cleaning street $\left(T_{1}, T_{2}\right.$ and $\left.T_{3}\right)$ and the rate of the surplus sludge pump $\left(T_{s t}\right)$. Figure 8.3 shows the probability that $\Phi_{A}$ holds for varying mean durations of rain between o.1 hour (6 minutes) and 4 hours. This parameter is the same for all four 3 D-plots, and is depicted on the $x$-axis, while the parameters on the $y$-axis are different, as mentioned above. All the other characteristics of the model, which are not explicitly parametrized, keep their values according to Figure 8.2.

Figure 8.3a shows the influence of the capacity of the community buffer $\left(P_{c}\right)$, by varying its value from 5 to 30 (from right to left on the $y$-axis). By increasing this capacity, the probability that formula $\Phi_{A}$ holds increases. We observe that this increase is non-linear, especially for larger values of the capacity, we see a faster improvement. Furthermore, we observe that for long rain duration, even if we increase the buffer capacity to 30 , still we have more than 20 percent probability of not satisfying the survivability property $\Phi_{A}$. This means that enlarging the buffer capacity alone is not enough for avoiding the flood in the area.

Figure $8.3 \mathrm{~b}$ shows how the system survivability depends on the rate of the fresh sludge thickener pump, $T_{f t}$ which is parametrized from 0.25 to 5 (from right to left on the $y$-axis). It can be seen that, by increasing the rate of this pump, the probability for $\Phi_{A}$ to hold increases. Specially for long rain duration $(\mu=4)$ this increase can be observed well. The increase is steeper than in Figure 8.3a, hence, this pump plays a significant role; for larger values of its rate, e.g., larger than 3, even if it rains for more than four hours, formula $\Phi_{A}$ holds with probability one. The reason for this is that the overflowed sewage from the primary tank, $P_{p s}$ could be handled with a rate of at most 4 , the rate of intake into the

2 For computational purpose, we use $\epsilon$ as a positive close to zero constant. 


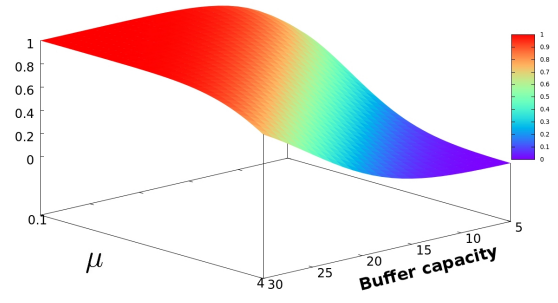

(a) The capacity of community buffer $\left(P_{c}\right)$

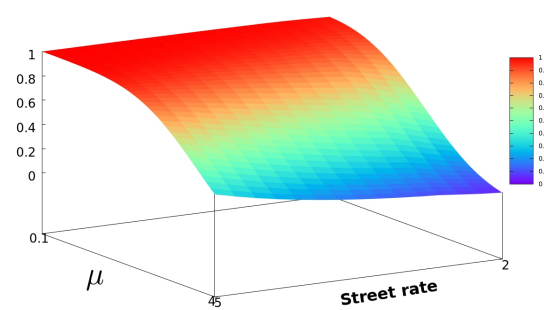

(c) The rate of the cleaning street pumps $\left(T_{1}, T_{2}\right.$ and $\left.T_{3}\right)$.

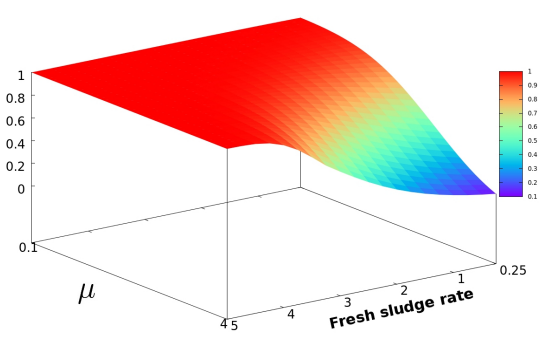

(b) The rate of fresh sludge pump $\left(T_{f t}\right)$.

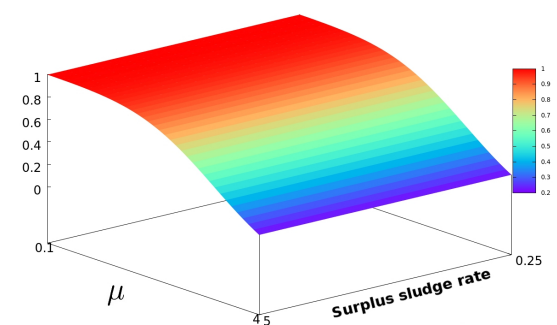

(d) The rate of surplus sludge pump $\left(T_{s t}\right)$.

Figure 8.3.: Probability for the survivability property $\Phi_{A}$ to hold, while varying different parameters of the model. $\mu$ is the mean rain duration, distributed according to a truncated Normal distribution with variance one.

cleaning street. So, the more we pump out of the primary tank, the more sewage intake the system can handle. However, since increasing the rate of this pump means pumping out sludge with more mixed water, this could be a disadvantage or even an obstacle for the next stage, i.e., sludge treatment.

Figure 8.3C shows the importance of the cleaning street pump rates, i.e., pumps $T_{1}, T_{2}, T_{3}$, of which we vary the rate from 2 to 5 (right to left). These pumps play a similar role as pump $T_{f t}$, but with lower impact. As can be seen, for long rain duration $(\mu=4)$ the survivability probability remains low. Like in the previous case, also here increasing the pump rates could be problematic, because raising the rates involves pumping out water mixed with more dirt, since there may not have been enough time for the dirt to settle down in the primary sedimentation tank.

Finally, Figure 8.3d, shows the influence of surplus sludge thickener pump $T_{s t}$. As can be seen, the rate of this pump has no effect on the survivability probability of the system. This can be explained by the fact that this pump plays a secondary role compared to the cleaning street pumps. Since the rates of pumps $T_{1}, T_{2}, T_{3}$ are constant, increasing 
any pump rate which is placed after them does not change the overall capacity of the system.

In order to generate each $3 \mathrm{D}$ graph in Figure 8.3, for each combination of parameters, one STD has to be generated, followed by a model checking procedure. Table 8.1 , shows the number of points in each $3 \mathrm{D}$ graph in Figure 8.3 and the overall computation time for producing that $3_{\mathrm{D}} \mathrm{graph}$, i.e., generating all the STDs and the model checking. As can be seen, even for this large case study, generating and model checking Iooo STDs takes less than a second! This clearly shows the value and efficiency of our method. 3

Table 8.1.: Overall computation time for generating results depicted in the $3 \mathrm{D}$ graphs of Figure 8.3.

\begin{tabular}{c|c|c} 
& Number of points & Computation time $(\mathrm{ms})$ \\
\hline Figure 8.3a & 1000 & 522 \\
Figure 8.3b & 800 & 573 \\
Figure 8.3c & 600 & 269 \\
Figure 8.3d & 800 & 459
\end{tabular}

\subsubsection{Scenario B: Failure in sand interceptor}

For the second scenario, we consider a failure in the sand interceptor pump, $T_{z}$, modelled by the deterministic transition $T_{f}$, firing at time $\alpha$, which again could be parametrized for any arbitrary value. After the occurrence of a failure, a repair crew will repair the pump with a duration distributed according to an exponential distribution, with mean 2 hours. For this case we investigate almost the same formula as before, only now the recovery condition is that the pump should be repaired:

$$
\Phi_{B}=\left(x_{P_{o}}<\epsilon\right) \mathcal{U}^{[\alpha, \alpha+30]}\left(m_{P_{r}}=1\right),
$$

where, $m_{P_{r}}=1$, means that the sand interceptor pump is repaired. Here, we have chosen the time bound $[\alpha, \alpha+30]$ for the Until operator, since the pump is supposed to be repaired within 30 hours after its failure.

For this scenario, we consider two parameters, the time of failure and the intake rate. The result is shown in Figure 8.4. On the $x$-axis the intake rate is parametrized from 6 to 13 , and the $y$-axis represents different times of failure, from 30 minutes to 5 hours (right to left). As expected, for larger rates of intake, the probability that survivability property $\Phi_{B}$ holds decreases. However, it is interesting that for a late occurrence of the failure, the probability is lower, especially for high intake rates. The

3 All computations in this section are done on a laptop equipped with a $2.0 \mathrm{GHz}$ intel ${ }^{\mathbb{R}}$ $\mathrm{CORE}^{\mathrm{TM}}$ i7 processor, and $4 \mathrm{~GB}$ of RAM. 
reason for this is that the capacity of the system is equal to the sum of the cleaning street rate pumps (4) and the fresh sludge thickener pump rate (1.25), which is 5.25. Therefore, the buffer is filling up for intake rates greater than 5.25 , and a late failure will cause a quicker violation of the safety condition. On the other hand, for early failures, we have a non-zero survivability probability, even for high intake rates.

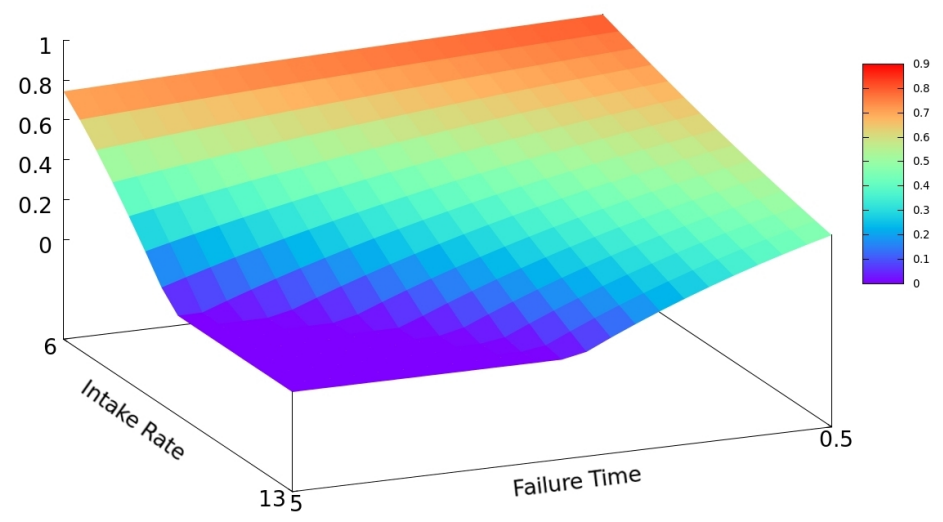

Figure 8.4.: Probability of survivability property $\Phi_{B}$ to hold for varying intake rate ( $x$-axis) and failure time ( $y$-axis).

Figure 8.5 provides a better understanding of this case. Each curve in this figure represents the survivability probability (y-axis) for a given fixed intake rate (color) to the system; the horizontal axis depicts the failure occurrence times. The time that the probability hits zero is the very moment that the community buffer has become full, hence, if the failure occurs at any time after that, the surrounding area will be flooded immediately. This is the reason that this probability equals zero for any time of failure after this point.

The two last figures show the importance of fast maintenance in bad weather conditions, otherwise, soon after the occurrence of a failure, flooding of the surrounding area is inevitable.

In addition to these results, the model checking algorithms can compute the set of satisfaction intervals, as also explained in Section 6.2.5. For the current case-study these satisfaction intervals represent the time intervals in which the repair can be done without violating the STL property $\Phi_{B}$. These intervals arise naturally from the model checking procedure and are used to compute the probability for a certain STL property to hold, as explained in Definition 4.6.

Table 8.2 shows these intervals for different intake rates, $f_{i}$, and failure times, $\alpha$. Note that these intervals are given relative to the failure time $\alpha$. This means, for instance, if there is a failure in the system at 


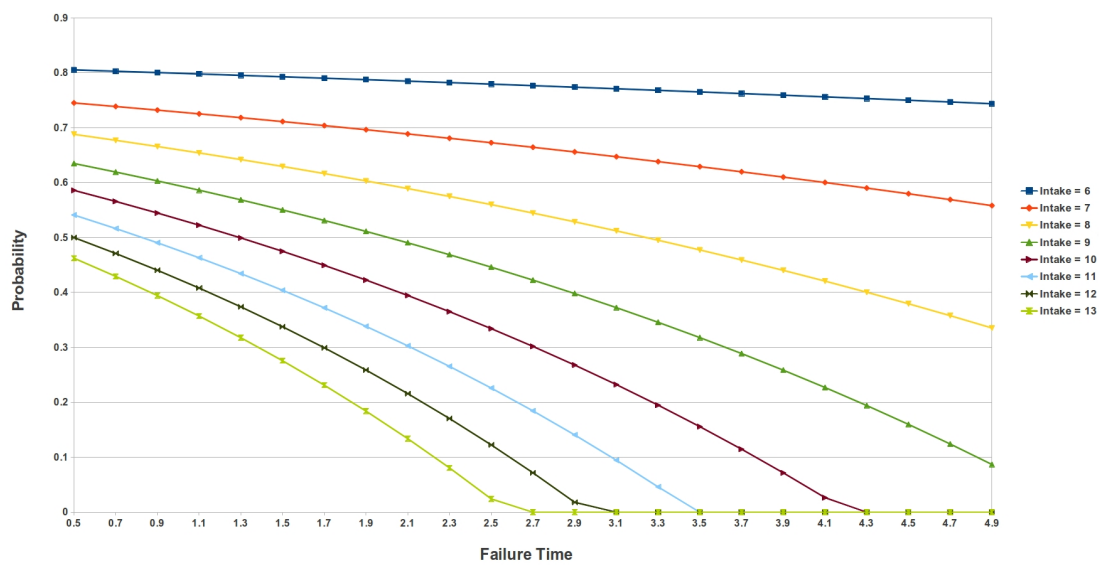

Figure 8.5.: Probability for survivability property $\Phi_{B}$ to hold. Each curve represents a specific intake rate, and the horizontal axis depicts the failure occurrence time.

$\alpha=3$, while the intake rate is 7 , the repair crew will have 2.109 hours to repair the pump, otherwise the safety condition in formula $\Phi_{B}$ is violated and the water is spilled on the street. Moreover, as can be seen, as the intake rate increases the repair crew will have less time to conduct the repair, especially for later failure times, if at all. This knowledge is specifically useful for scheduling repair crews, and planning emergency maintenance processes.

\begin{tabular}{|c|c|c|c|c|c|c|c|c|}
\hline$\alpha$ & 6 & 7 & 8 & 9 & 10 & 11 & 12 & 13 \\
\hline 1 & {$[0,3.210]$} & {$[0,2.609]$} & {$\left[0,2.15^{8}\right]$} & {$[0,1.807]$} & {$[0,1.526]$} & {$[0,1.296]$} & {$[0,1.105]$} & {$[0,0.943]$} \\
\hline 2 & {$[0,3.085]$} & {$[0,2.359]$} & {$[0,1.814]$} & {$[0,1.390]$} & {$[0,1.051]$} & {$[0,0.774]$} & {$[0,0.542]$} & {$[0,0.347]$} \\
\hline 3 & {$[0,2.960]$} & {$[0,2.109]$} & {$[0,1.470]$} & {$[0,0.973]$} & {$[0,0.576]$} & {$[0,0.251]$} & $\varnothing$ & $\varnothing$ \\
\hline 4 & {$[0,2.835]$} & {$[0,1.859]$} & {$[0,1.126]$} & {$[0,0.557]$} & {$[0,0.101]$} & $\varnothing$ & $\varnothing$ & $\varnothing$ \\
\hline 5 & {$[0,2.710]$} & {$[0,1.609]$} & {$[0,0.782]$} & {$[0,0.140]$} & $\varnothing$ & $\varnothing$ & $\varnothing$ & $\varnothing$ \\
\hline
\end{tabular}

Table 8.2.: Satisfaction intervals for different failure occurrence times $\alpha$ and intake rate $f_{i}$.

\subsection{MULTiple GENERAL TRANSitions: DiscRETizATION}

In this section we exploit the idea of discretization, as explained in Section 7.1 for the analysis of HPnG models with more than one general transitions to analyse a setting where both scenarios, namely A and B, are present simultaneously. Firstly, we consider an overall investigation of the system to show the feasibility and efficiency of the proposed method, and secondly we will discuss discretization of each general tran- 
sition separately to provide an insight in how one can decide discretization of which of the general transitions leads to a better performance. Moreover, as mentioned in Section 7.1 we will investigate performance of different methods of discretization, namely naive discretization and Monte Carlo method.

\subsubsection{Feasibility and efficiency}

We start the system analysis in bad weather conditions (sewage production with rate 12.2) and the sand interceptor pump, $T_{z}$, fails at a certain point in time $\alpha$, followed by a stochastic repair. Again, we want to know the probability that $\Phi_{A}$ (Equation 8.1) holds, since we are interested to not have sewage water in the streets during bad weather.

We discretize the general transition $G_{b}$ which models the stochastic repair process, with discretization step o.1. We investigate the influence of two parameters, i.e., the mean duration of rain, and the failure time of the sand interceptor which is modelled by the firing of the deterministic transition $T_{f}$. Again we assume that the duration of the rain is distributed according to the normal distribution with standard deviation $\sigma=1$, and mean $\mu$ between 0.1 and 4 , as in Scenario A. The repair process is modelled by a Gamma distribution with parameters, $K=4$ and $\theta=1 / 2$, which means that the repair process has a mean duration of 2 hours. ${ }^{4}$ The result of the analysis is shown in Figure 8.6.

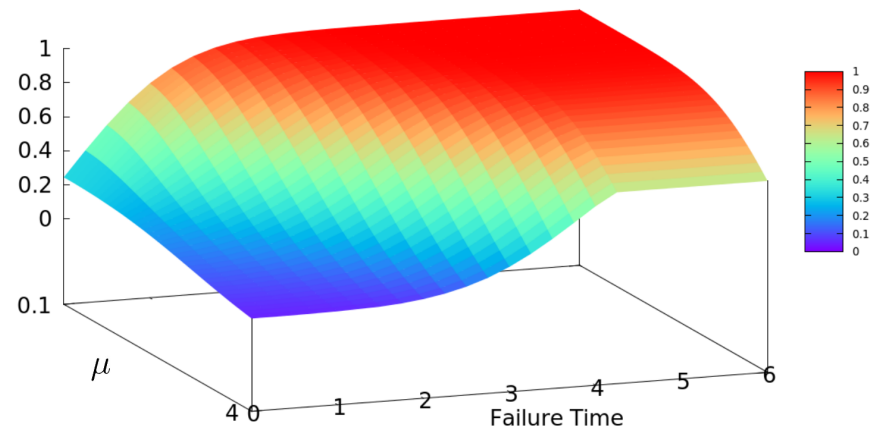

Figure 8.6.: Probability of survivability property $\Phi_{A}$ to hold for varying mean duration rain $\mu$ ( $x$-axis) and failure time ( $y$-axis).

Figure 8.6, like scenario B, shows that the later the failure takes place, the more vulnerable is the system. The reason for this is that the com-

4 Again the choice of probability distributions are arbitrary, and can be replaced by any other distribution. 
munity buffer fills up quickly in case of bad weather, hence, late failures often have to cope with an already full community buffer. Moreover, it can be seen that slightly after 4 hours, the failure time has no impact on the probability. This is because at that time the sewage has already slipped into the streets, since the community buffer is already filled by the increased sewage production of rain.

This diagram consists of 960 data points, for each of which the discretization of the second general transition is performed. This means that for each point, 400 STDs (discretization step of 0.1 and maximum analysis time of 40) are generated and model checked. Hence, for the generation of this diagram, $960 \times 400=38000$ times the process of STD generation and model checking is performed. However, the total computation time does not exceed 50 seconds. This clearly shows the power of the proposed method.

\subsubsection{Discretization methods}

In this section we show how using the Monte Carlo (MC) method [94] for discretizing the support of general transitions increases the performance of the proposed method. We also investigate how one can choose one general transition over another for discretization, and how the shape of probability distributions influences the convergence rate.

For this section we again start the analysis in bad weather conditions (sewage production with rate 12.2), but with an already failed sand interceptor pump, $T_{z}$, i.e., $T_{f}$ fires at time zero. As in Section 8.3.1 we compute the probability for $\Phi_{A}$ to hold. For this section we assume that $G_{a}$ fires according to a Gamma distribution, with parameters defined later, in order to show how the shape of probability distribution influences the convergence rate. We here assume that $G_{b}$ fires according to a Chi-squared probability distribution with 2 degrees of freedom ${ }^{5}$.

Figure $8.7 \mathrm{a}$ and $8.7 \mathrm{~b}$ each shows four different discretization results. In each figure both $G_{a}$ and $G_{b}$ are discretized once with naive steps and once with the MC method. We have used a Gamma distribution with different parameters for $G_{a}$, namely $\operatorname{Gamma}(2,1)$ (Figure 8.7a) and $\operatorname{Gamma}\left(2^{7}, 2^{-6}\right)$ (Figure 8.7b), in which the first and second parameters are shape and scale parameters, resulting in the same average of 2 for both distributions. Figure 8.8, shows probability density function for these two distributions. As one can see although both have the same mean value, however, $\operatorname{Gamma}\left(2^{7}, 2^{-6}\right)$ depicts much lower variance that $\operatorname{Gamma}(2,1)$. We will later discuss how the shape of probability distribution influences the convergence of discretization methods.

5 The probability distributions are chosen to show the effect of their shape on the analysis, however, any probability distribution with positive support can replace them. 


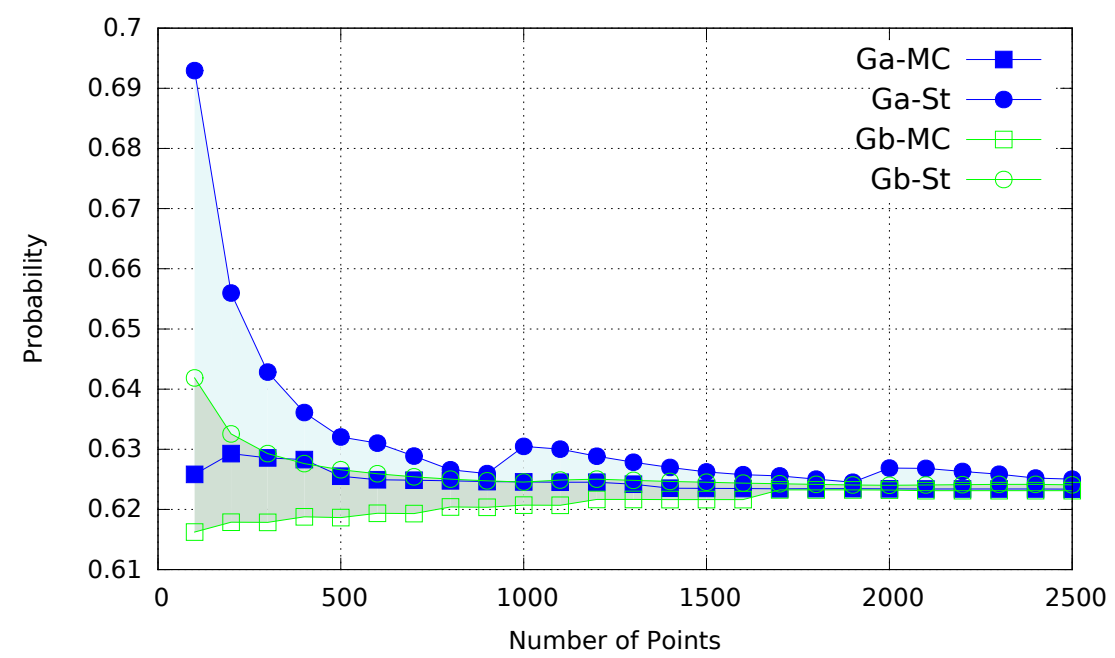

(a) $G_{a} \sim \operatorname{Gamma}(2,1)$

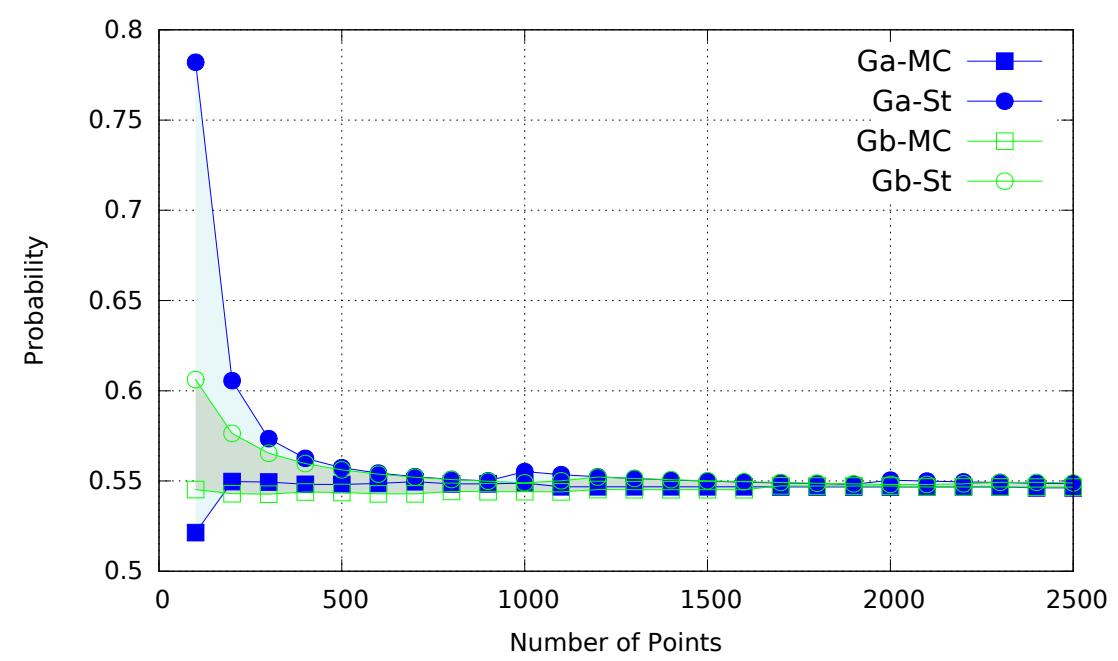

(b) $G_{a} \sim \operatorname{Gamma}\left(2^{7}, 2^{-6}\right)$

Figure 8.7.: Comparison of discretization methods, constant steps and Monte Carlo (abreviated as St and $\mathrm{MC}$ in the figures repecively), by computing the probability that property $\Phi_{A}$ holds. 


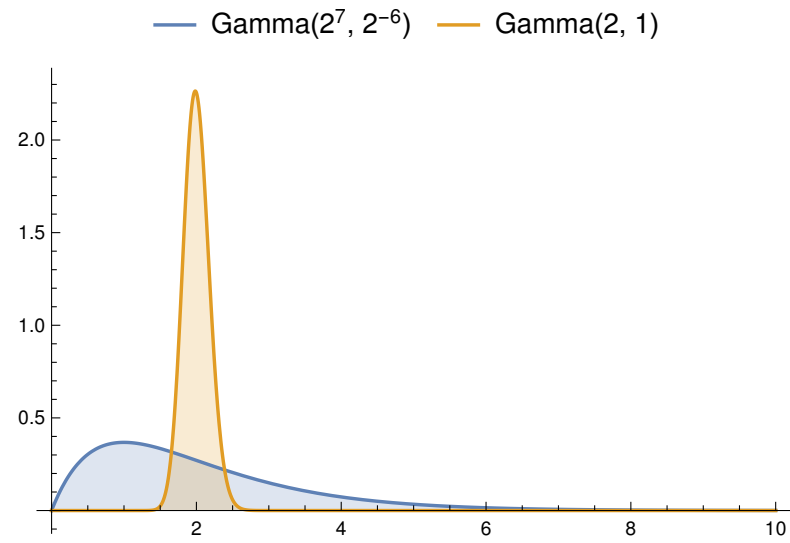

Figure 8.8.: Probability density function for $\operatorname{Gamma}(2,1)$ and $\operatorname{Gamma}\left(2^{7}, 2^{-6}\right)$

The horizontal axis in Figure 8.7, represents the number of discretization points, and the vertical axis shows the probability that formula $\Phi_{A}$ holds. One can see that in both cases the MC method results in faster convergence to the real value of the probability for both general transitions. However, we can also observe that discretizing $G_{b}$ performs better in comparison to $G_{a}$, as it is closer to the final value, for each number of discretization points. In order to understand this we need to take a closer look at the structure of the discretized area of integration.

As mentioned earlier, when we are discretizing the support of a general transition, say $G_{a}$, we treat it as a deterministic transition firing at each discretization point. Then for each of these points, we find the satisfaction interval for the other general transition $G_{b}$. According to Equations (7.1) and (7.3), when we have computed a satisfaction interval for one discretization point, say $s_{i}$, we assume that all points between $s_{i}$ and the next discretization point, $s_{i+1}$, have the same satisfaction intervals as $s_{i}$. This means that we are approximating the final integration area (for computing the probability of a given formula) with a set of rectangles. This is illustrated schematically in Figure 8.9, by showing the approximated rectangular areas (blue) and the real area (red). One can observe that by increasing the number of discretization points we will have a better approximation of the real area.

Figure 8.10 shows the approximation of the integration area, for the case-study, for three different numbers of discretization points of $G_{a}$. In each figure the discretization points are indicated by red crosses at the $x$ - 


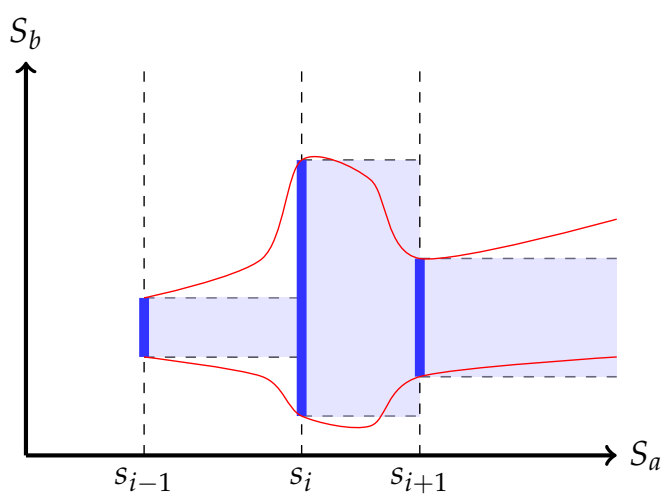

Figure 8.9.: Showing the approximation of the true integration area (between the red curves) by discretizing $S_{a}$. The satisfaction interval for each discretization point is shown by a thick blue line, and the resulting approximation as shaded blue.

axis. ${ }^{6}$ For instance, in Figure 8.10a at point o, i.e., $G_{a}$ firing at time o, the satisfaction interval is the entire support of $G_{b}$ (y-axis), while at the next points, i.e., 4 and above, the satisfaction intervals are empty. As can be seen by increasing the number of discretization points in Figures 8.10b and 8.10c, the resulting area shrinks to a more precise approximation of the real area. Moreover, one can observe that when $G_{a}$ fires after, approximately, time point 3, the satisfaction intervals of $G_{b}$ are empty, and $G_{a}$ has a smaller area of influence in comparison to $G_{b}$. This is the reason that discretizations of $G_{a}$ for small numbers of discretization points results in poor performance, since we need more points, i.e., a small discretization step, to observe this small area.

Knowing the above fact, we can conclude that if the probability distribution associated with $G_{a}$ takes most of its mass before time point 3, the convergence is fast. This is the case when we use $\operatorname{Gamma}\left(2^{7}, 2^{-6}\right)$ (Figure $8.7 \mathrm{~b}$ ). On the other hand, if we use $\operatorname{Gamma}(2,1)$, since considerable amount of probability concentrates after time point 3 , the rate of convergence is slow (Figure 8.7a). However, since the MC method chooses points before time 3 with higher probability, because of the shape of the density function $\operatorname{Gamma}(2,1)$, it results in a better convergence. Figure 8.11 illustrates these facts by showing both approximated integration areas (as have already been computed and shown in Figure 8.10) plus the considered probability density functions $\operatorname{Gamma}(2,1)$, and $\operatorname{Gamma}\left(2^{7}, 2^{-6}\right)$.

6 The maximum time of analysis for this case is 40 , however, we are showing the range of general transitions up to 8 , for sake of visibility. 


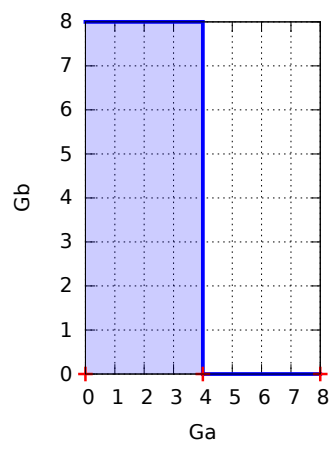

(a) 10 discretization pts

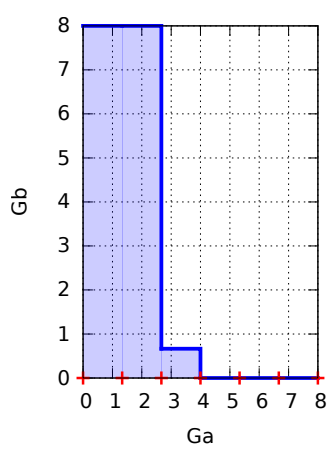

(b) 30 discretization pts

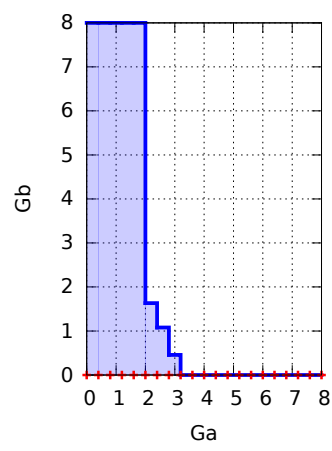

(c) 100 discretization pts

Figure 8.10.: Resulting areas by discretizing support of $G_{a} . x$-and $y$-axes are the supports of $G_{a}$ and $G_{b}$, respectively.

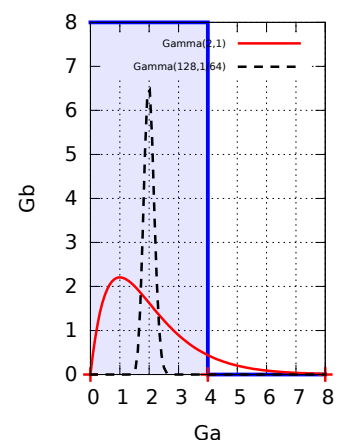

(a) 10 discretization pts

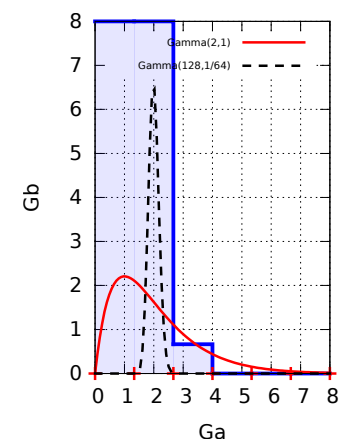

(b) 30 discretization pts

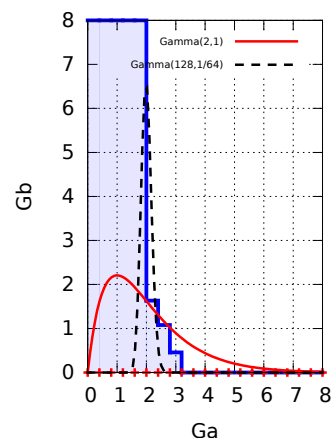

(c) 100 discretization pts

Figure 8.11.: Demonstrating how the shape of the probability density function and the number of discretization points can influence on the performance of the method. 


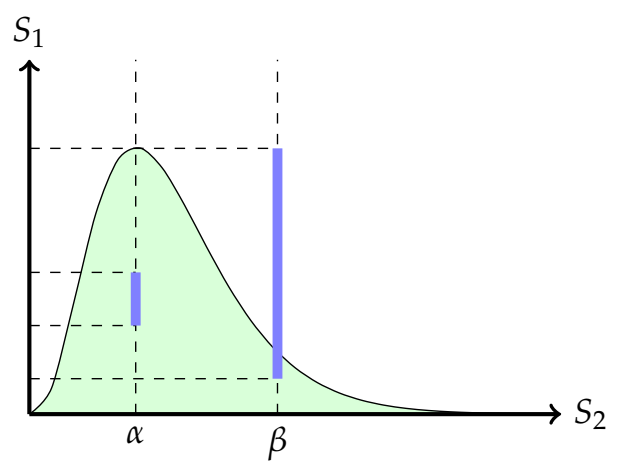

Figure 8.12.: Showing the satisfaction intervals for two different values of $S_{2}$.

This provides us with useful insight in order to decide which transition to choose for discretization, prior to the start of analysis. For this, one can generate the approximated integration area with few discretization points as in Figure 8.10. Then based on the structure of the area and the shape of the associated probability distributions, choose which general transition to discretize. This is indeed why, in Section 8.3.1, we have chosen $G_{b}$ for discretization.

Finally, it is important to note that the MC method will not always lead to better performance. This can be observed in Figure 8.7a, for discretization of $G_{b}$, since the MC method does not show an obvious increase in performance. The MC method guarantees that we are maximizing the term $P_{S_{2}}\left(S_{2}=s_{2}^{(i)}\right)$ in Equation (7.1), however, the other term, i.e., $\operatorname{Prob}^{t}\left(\Psi \mid S_{2}=s_{2}^{(i)}\right)$, depends on the resulting satisfaction intervals by fixing firing time $S_{2}$ for the second general transition. Figure 8.12, is schematically demonstrating this fact by showing the corresponding satisfaction intervals of $S_{1}$ by fixing $S_{2}$ to two values of firing times, namely, $\alpha$ and $\beta$. These satisfaction intervals are depicted by thick blue lines. Moreover, the probability distribution of $S_{2}$ is shown. Based on this assumed distribution, we have $P_{S_{2}}\left(S_{2}=\alpha\right)>P_{S_{2}}\left(S_{2}=\beta\right)$. However, although for firing time $S_{2}=\alpha$ we have the highest probability, the resulting satisfaction interval is smaller compared to the satisfaction interval of firing of second general transition at $S_{2}=\beta$. Therefore (depending on the shape of probability distribution of $S_{1}$ ), one may have $\operatorname{Prob}^{t}\left(\Psi \mid S_{2}=\alpha\right)<\operatorname{Prob}^{t}\left(\Psi \mid S_{2}=\beta\right)$. This means preferring points like $\alpha$ over points like $\beta$ does not necessarily lead to maximization of summation (deconditioning) in Equation 7.1. 


\subsection{RELATED WORK}

There are plenty of simulation packages and tools for analysis of water treatment facilities [107, 108]. The difference of our approach in this chapter relies on the conventional difference of simulation methods and analytical methods. Although the former provides the possibility of treatment of a wider range of application areas and imposes less constraint on the model, the advantage of the latter is that it equips us with exact analysis, e.g., exact values for probabilities of certain properties. If some rare events are associated with very high costs/risks, such as the failures that we considered in this chapter, analytical methods are highly advantageous since they can compute small probabilities of occurrence of such events. However, rare events in a simulation are usually not observed even after a very large repetitions of simulations.

We should mention that, there are techniques for simulation of rare events, such as extreme value theories [109], which provide the possibility of estimating the probability of rare events. However, these methods are in nature different from exact analysis. 


\section{CONCLUSIONS}

In this dissertation we considered hybrid Petri nets as a high level modelling tool for systems with hybrid characteristics. As we mentioned throughout this thesis, one of the main application areas we were aiming for is dependability analysis of critical infrastructures. We counted key characteristics of such systems, including the possibility of occurrence of discrete random events with arbitrary probability distributions, and also the complexity of these systems in terms of number of continuous variables. The first characteristic demands for integrating stochasticity inside hybrid Petri nets, and the second characteristic imposes the requirement for high performance of solution methods. Furthermore, we discussed that since usually a high cost is involved in failure of critical infrastructure, it is important to be able to provide exact analysis results for safety questions regarding these systems.

Having said this, we tackled two restrictions in analysis methods using hybrid Petri nets. The first restriction is related to arbitrary nature of occurrence of discrete events, and the second restriction relates to the number of possible continuous variables (modelled by continuous places). As we discussed in Section 3.6, the existing solution methods for Petri net formalisms, suffer from either of these limitations. For the first restriction, i.e., in order to allow the occurrence of random discrete events, we included general transitions in the modelling formalism of hybrid Petri nets. The firing times of these transitions is sampled according to arbitrary probability distributions. The second restriction is related to the performance of the analysis methods, (the model checking algorithms). To overcome this restriction we introduced Stochastic Timed Diagrams (STDs). The main idea behind STD is to transform the state space of infinitely many points into finitely many regions, for which model checking algorithm over a finite time horizon can be performed. As we discussed, by providing several case studies, STDs provide the possibility of computing exact probability for given system properties, in an efficient way.

HPnGs also suffer from some limitations. The most important one is the limitation of the number stochastic variables, i.e., discrete random events. This was (partly) due to lack of implementation for some of 
the underlying computational geometry algorithms. However, we also tackled this limitation by providing two approximation techniques. Both of these techniques are based on discretizing the support of probability distributions according to which the firing times of general transitions are being sampled.

Furthermore, we used our proposed solution method for two real world applications. We analyzed the survivability of a sewage treatment facility, and considered energy resilience modelling for smart houses (included in the appendix).

In the remainder of this short concluding chapter we revisit the research questions given in Chapter 1 , and address where and how each of them has been answered in this thesis. After that, we conclude by providing possible lines for future work.

RESEARCH QUESTIONS REVISITED

The first research question was formulated as:

$\mathcal{Q} 1$ How can arbitrary probability distributions that describe the occurrence of discrete events, be incorporated in hybrid Petri nets, and yet be accompanied by efficient and exact techniques?

For answering this question, in Chapter 3 we discussed in detail the formalism of Hybrid Petri nets with General transitions (HPnGs), which is indeed the extension of hybrid Petri nets, by providing the possibility of occurrence of random discrete events, with arbitrary probability distributions. For the solution method and for computing measures of interests, we introduced Stochastic Timed Diagrams (STDs) as a smart representation of their underlying state space. The idea of generating STDs has been based on grouping infinitely many points into finitely many partitions, in a given finite time horizon. This smart compact representation followed by a deconditioning argument, provided us with the possibility of efficient and exact computation of measures of interest, as was the requirements stated in $\mathcal{Q} 1$. The theoretical developments for this approach were done mainly in Chapters 5 and 6, and supported by Chapter 8, and Appendix A, for real-world applications.

The second research question, $\mathcal{Q} 2$, was about the limitations of the proposed method: 
Q2 What are the limitations of the modelling formalism and analysis methods devised in response to $\mathcal{Q}_{1}$ ? In other words, how far can the complexity of the proposed model, in terms of the number of stochastic and continuous variables be pushed, considering efficiency and exactness?

Although the mentioned restrictions regarding existing Petri nets solution methods are removed, the analysis methods proposed for HPnGs do still impose restrictions on other aspects of the modelling power, as follows:

1. The law governing the evolution of continuous variables in HPnGs is described by constant differential equations, i.e., continuous variables evolve linearly in time.

2. The number of stochastic variables, i.e., random discrete events, for which exact analysis can be provided is restricted.

Therefore, $\mathcal{Q} 2$ is answered by the fact that the complexity of the provided solution method for HPnGs, is not limited by the number of continuous variables, however, it is limited by the number of discrete random events. Having said this, placing HPnGs in the context of Table 3.3, from Section 3.6, to be compared with the existing formalisms, results in Table 9.1. For description of solution methods mentioned in this table we refer to Section 3.6.

Table 9.1.: Comparison of HPnGs with different formalism and solution methods of Petri net for handling continuity and stochasticity.

\begin{tabular}{c|c|c} 
Models & \# Continuous places & Stochasticity \\
\hline \hline SPN, GSPN & No & Only exponential \\
\hline $\begin{array}{c}\text { MRSPN, Method of } \\
\text { supplementary variables }\end{array}$ & No & Arbitrary \\
\hline $\begin{array}{c}\text { FSPN } \\
\text { supplementary variables }\end{array}$ & Yes, but limited & Only exponential \\
\hline HPnG & Yes, no limitation & $\begin{array}{c}\text { Arbitrary, but limited } \\
\text { in number of events }\end{array}$
\end{tabular}

Important note regarding Table 9.1, is how HPnGs are compared to existing formalisms and solution method. As can be seen the main advantage of HPnGs is that it does not impose a limit on the number of continuous variable (second column) as all the existing methods do. However, when it comes to stochasticity (third column), although it allows arbitrary probability distributions for random events, it is limited in the number of occurrence of these events. 
In spite of these restrictions, as we discussed with several case-studies and real-world applications, HPnGs are suitable for certain applications, such as dependability analysis of critical infrastructures. This brings us to the third and the last research question, $\mathcal{Q} 3$ :

$\mathcal{Q}_{3}$ In light of $\mathcal{Q}_{2}$, can we develop algorithms to provide approximate results for more complex systems?

We have answered this question by tackling the limitation regarding random events, by providing two approximation techniques, both of them based on the merits of discretization. The first method allows us to keep one random event to have a continuous support, and is still based on the idea of deconditioning. The second method follows a completely different approach, based on graph search, and does not allow continuous support for discrete random events any more. These methods are discussed in Chapter 7, and their feasibility is investigated through case studies in the same chapter, and also in Chapter 8.

At the end of this section we conclude with a table summarizing all the model checking approaches introduced in this thesis. Table 9.2, is categorizing different methods into two main categories (exact and approximate), and demonstrates what sort of model checking for each of them can be carried out. Also, as discussed in Chapter 6, we developed model checking algorithm of HPnGs for two different cases: for statebased formulas, and for Until formula. In Table 9.2, for each model checking method, it is shown whether it is implemented for each of the two mentioned case. We stress that, those methods for which the model checking algorithm has not been implemented, can be considered as future work, as the theoretical development for all of them are discussed in this thesis.

\section{POSSIBLE LINES OF FUTURE WORK}

In general, analysis of stochastic hybrid systems encompassing both hybrid characteristics and stochasticity, is known to be computationally expensive, or even undecidable. Therefore, any solution method approaching these problems should have an eye towards the application domain it is aiming for, and based on that restrict the different aspect of modelling features. The solution method provided in this dissertation is no exception to that. As discussed throughout this thesis, one of the application domains we were aiming for, was dependability analysis of fluid critical infrastructures. For the analysis of such systems, restrictions on the number of continuous variables are not acceptable. However, the 
Table 9.2.: Comparison of different methods for model checking HPnGs.

\begin{tabular}{|c|c|c|c|}
\hline \multicolumn{2}{|c|}{1} & State-based & Until \\
\hline \multirow{3}{*}{ 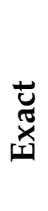 } & One stochastic variable & Yes & Yes \\
\hline & Two stochastic variable & Yes & No \\
\hline & Multiple stochastic variable & No & No \\
\hline \multirow{2}{*}{$\begin{array}{l}\frac{x}{0} \\
\frac{0}{2} \\
\frac{2}{2}\end{array}$} & Discretization & Yes & Yes \\
\hline & PTTs & Yes & No \\
\hline
\end{tabular}

number of random discrete events could be limited, since these usually model unknown failures or repair, whose occurrence over a given time span could be limited.

As we discussed before, formalization of HPnGs in Chapter 3 was general enough to support multiple general transitions, with multiple firings. However, the exact solution method introduced in Chapters 46 was limited in the number of firings of general transitions. Indeed, the proposed algorithm did allow only two firings. This was related to the lack of implementation for the underlying computational geometry algorithms (specifically, hyperplane arrangements). Technically, the number of regions in an STD grows exponentially with the number of firings of general transitions. But still, from a practical point of view, and considering the efficiency of the proposed algorithm in this thesis, and the importance of the application domain, having an exact solution method that can allow more than two firings is indeed advantageous. Therefore, one line for future work is to fill in the gap by implementing the hyperplane arrangement algorithm for higher dimensions.

As mentioned above, in HPnGs the evolution of continuous variables, i.e., the content of continuous places is linear. This is the main reason why the boundaries between regions in the generated STD are consisting of lines, planes, and hyperplanes in higher dimensions. Because of this limitation, HPnGs are mostly suitable for networks of fluid, for which usually constant differential equations are applicable for representing their evolution over time. However, analysis of networks allowing more complex evolution, e.g., gas networks, are no longer possible since the laws governing the evolution of the continuous variables are no longer described by constant differential equations. In this case the regions in the STD cannot be described by simple polytopes, and the boundaries would be curves in 2 dimensions, and general surfaces in higher dimen- 
sions. In the simplest possible form, for the case of linear first-order differential equations, we will see exponential curves. One line for future work is to see how these curves can be handled. It would be of interest to see if it is still possible to compute measures of interest exactly, and if not, in case of using numerical methods, e.g., for representation for curves and surfaces, what sorts of approximation techniques can be used. 
Part IV

APPENDIX 



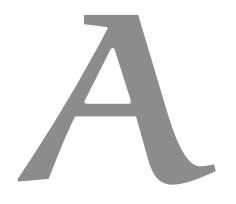

\section{ENERGY RESILIENCE MODELLING FOR SMART HOUSES}

The content of this chapter is based on:

[110] H. Ghasemieh, B. R. Haverkort, M. R. Jongerden, and A. Remke. Energy resilience modelling for smart houses. In 45th Annual IEEE/IFIP International Conference on Dependable Systems and Networks, pages 275-286. IEEE, 2015 .

Over the last decade, we have seen an increasing amount of locally generated energy in households. The decrease in costs of photovoltaic (PV) panels, and governmental subsidies for solar energy has lead to a huge increase of distributed generation with PV-systems. Especially in Germany, the national government has been very successful in increasing the number of installed PV-power [1II]. Even though this provides a large contribution to the country's generation of renewable energy, the large increase of distributed generation has its drawbacks as well, as it leads to enormous production peaks. At sunny days the amount of generated solar energy becomes so large that it is a serious thread to grid stability.

These problems can be overcome through grid balancing, which, however, requires the possibility to store locally generated energy. The German government, for example, subsidises investments in battery storage for solar energy [111]. Local storage reduces the amount of power that is exported to the grid at peak production times, hence, leads to a better balanced and more stable grid.

Furthermore, investing in local storage facilities also allows to increase the resilience of smart houses and neighbourhoods, since the local storage can provide back-up power to isolated units in case of grid failures. Commercial systems with both of these functionalities, increasing local usage and providing back-up power, are available on the market, e.g., Nedap's PowerRouter [112] or SMA's Sunny Island [113]. Although the back-up power may not always serve as an uninterrupted power sup- 
ply (UPS), in the context of smart houses it is already very important to ensure that power is available after a short switching period.

This chapter presents an HPnG model of a smart house with local storage, adaptable production and demand patterns per hour, and a battery management unit that allows to implement different strategies for balancing the use of battery and the grid. As a quantitative measure of resilience that focuses on the effect of interruptions over time, we analyse the survivability of the smart house, given the presence of a power outage. The survivability in this context specifies the probability that the house can be continuously powered from the locally produced and stored energy during a grid failure. Clearly, this probability highly depends on the current production and demand profile, the time of day the failure occurs, and the duration of the failure.

We analyse a wide range of scenarios with realistic production and demand patterns (obtained from [114] and [115]) and three different battery management strategies. Our analysis shows a clear trade-off between the improved resilience against grid failures on the one side and flexibility for balancing purposes on the other.

This chapter is structured as follows. Section A.1 describes the system we analyse, provides the HPnG model for that, and introduces the strategies being used for balancing grid and battery. Section A.2 provides and discusses the results of our analysis for different scenarios in case of a grid failure. In Section A.3 an overview of related work is given.

\section{A.I SYSTEM AND MODEL}

The system we consider is a house with PV-panels for energy generation and a battery for energy storage. Figure A.I shows the HPnG model of the house. The model consists of several components, which are marked by dashed boxes. We address these components in the following subsections.

\section{A.1.1 Battery}

Continuous place, $B$, in the center of Figure A.1, models the battery. The battery has two main functions. First, when the grid is available, it can be used for energy balancing. By smartly charging the battery with the locally generated energy and discharging at times when the local generation is low, the impact of the household on the grid can be reduced. The second function of the battery is to provide back-up power when the grid fails.

The partial usage of the battery, i.e., assuming that battery is not in its low state of charge reduces the impact of the non-linear properties [116]. Even at high discharge currents it will be possible to discharge the bat- 


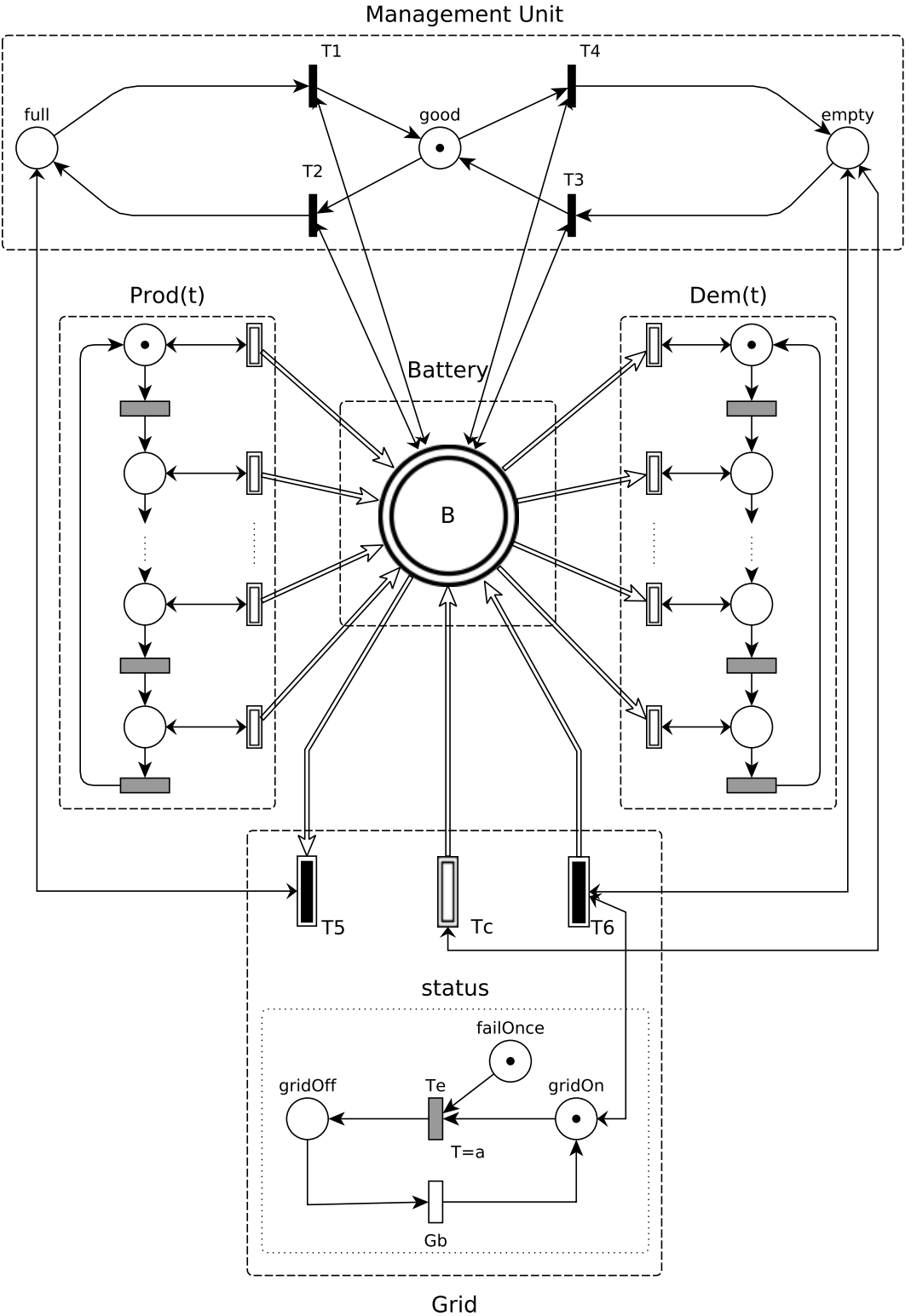

Figure A.1.: HPnG model of a resilient house. 
tery for the limited amount of energy. This means that we can approximate the battery with an ideal storage system without introducing large errors. We assume an ideal battery, where the change and discharge are always linear.

\section{A.1.2 Demand}

The energy demand of a household depends on the time of day, and the day of the year. Usually the electricity demand will typically be high in winter, and lower in summer. Figure A.2a, taken from [110], shows the average demand profile of a day both in summer and winter. However, to reduce the computational complexity for the HPnG model, we use a coarser approximation. The approximation profiles are given in Figure A.2b.

The demand profiles for a day are indicated with the dashed box $\operatorname{Dem}(\mathrm{t})$, in Figure A.1. As can be seen with use of deterministically timed transitions, the system switches between different demand rates for discharging the battery. As can be seen in the approximated demand profiles in Figure A.2b, (by counting the number of steps) we have 9 different demand rates, for both summer and winter. This is modelled using 9 continuous transitions in $\operatorname{Dem}(\mathrm{t})$ box, modelling different demand rates. Note that for each summer and winter demand profile, we have different HPnG model.

\section{A.1.3 Production}

The energy generation by the PV-panels, of course, mainly depends on the local weather. In general, the production will by high in summer and lower in winter. But large variations may occur on a day to day basis, since sunny days may be followed by days with many clouds. Figure A.3, taken from [110], shows four day profiles, two days in summer and two days in winter. For both seasons a profile of a sunny day with high production and a profile of a cloudy day with low production are shown.

In Figure A.1, the production profiles are modelled in the box $\operatorname{Prod}(\mathrm{t})$. Again by employing deterministic timed transitions the model switches between different production rates, to charge the battery. Note that for each production profile in Figure A.3, we need a different number of continuous transitions modelling the production rates. Hence, we need to consider a different HPnG model for each production profile.

\section{A.1.4 Grid and Management Unit}

The Management Unit (the top box in Figure A.1) controls the flow of power between the local generation, the battery, the house and the grid. 


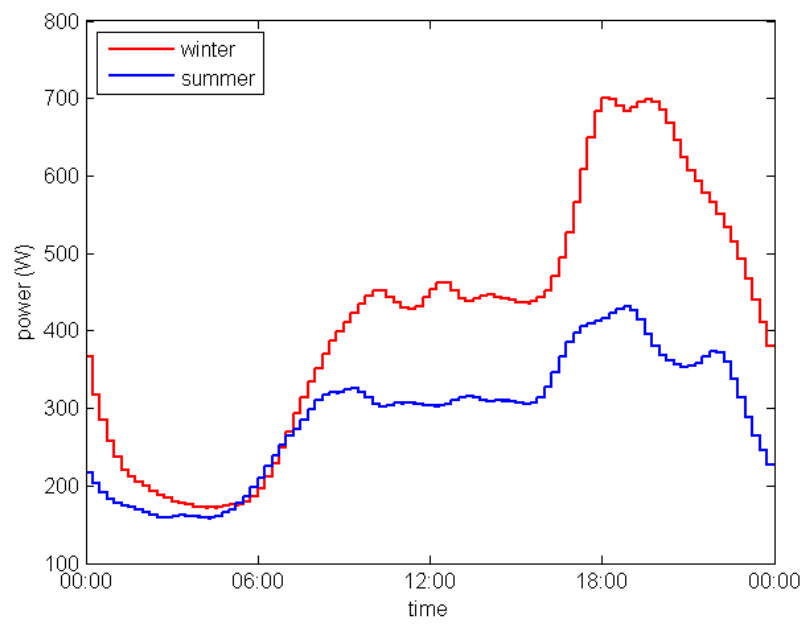

(a) Actual demand profiles.

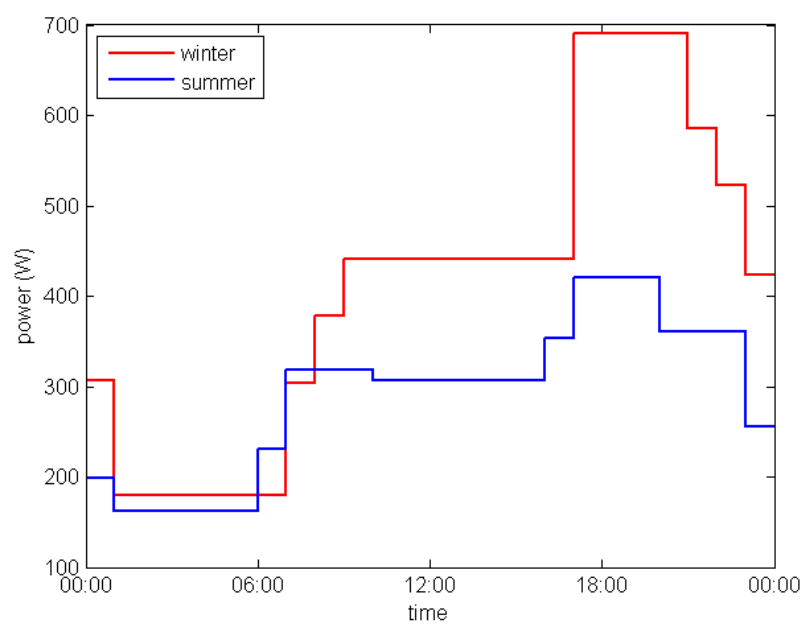

(b) Approximated demand profiles.

Figure A.2.: Summer and winter day demand profile. 


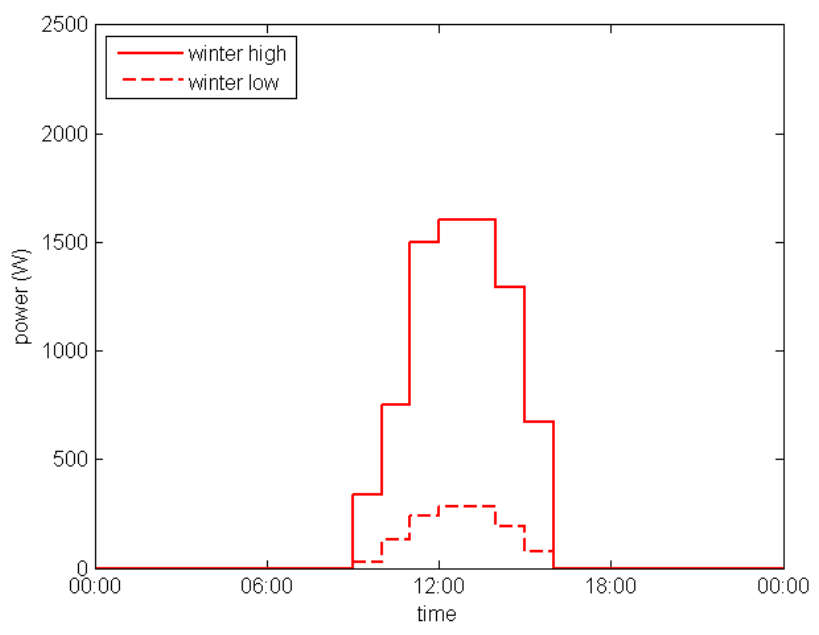

(a) Winter

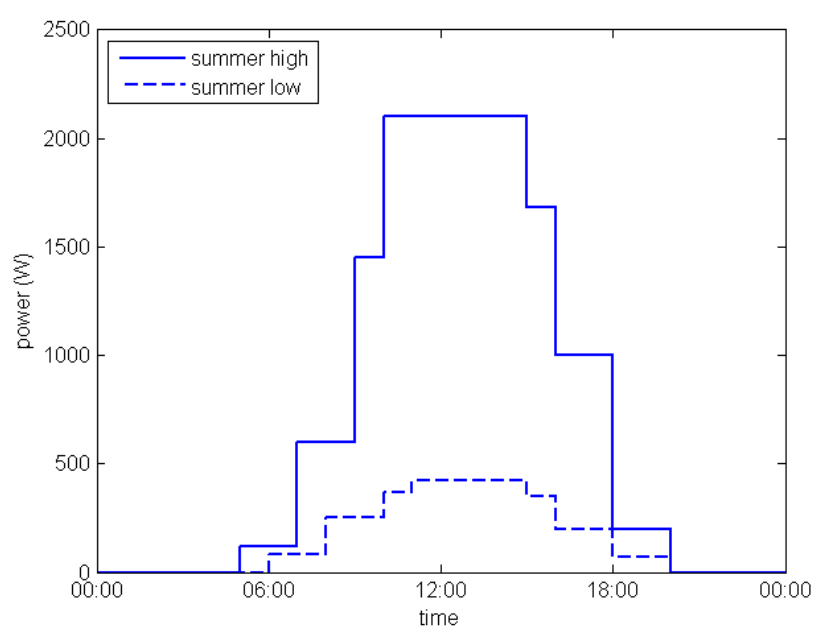

(b) Summer

Figure A.3.: Production profiles of winter and summer days. For both seasons a high and low production day are given. 
In case more energy is needed than is produced through local generation, it decides whether to take the additional power from the grid or from the battery, depending on the battery management strategy used. We consider three strategies. When the local production is smaller than the demand $(\operatorname{Prod}(t)<\operatorname{Dem}(t))$, the battery is used if possible. Only when really needed, the grid is used to supply the energy to the demand. The following strategies are considered:

i. Greedy: The battery is always discharged for its full available capacity. The battery is charged only with locally produced energy (no use of grid; which means the nominal rate of continuous transition $T_{\mathcal{C}}$ is set to zero, i.e., $\left.\phi_{S t}^{\mathcal{T}}\left(T_{\mathcal{C}}\right)=0\right)$.

ii. Smart: When the grid is available, the battery is never fully discharged. It is discharged only to a predefined state of charge, denoted $S_{o} C_{1}$. Part of its usable energy is kept as back-up energy. This energy is available when a grid failure occurs. Like the Greedy strategy, the battery is charged only with locally produced energy.

iii. Conservative: Like in the Smart strategy, the battery is discharged only to the level of $S_{o} C_{1}$ when the grid is available. However, when this state is reached, the grid is used to partially recharge the battery with a fixed rate $\phi_{S t}^{\mathcal{T}}\left(T_{\mathcal{C}}\right)=I$, to $S_{o} C_{2}$. This results in additional backup energy, when a grid failure occurs.

In order to implement these strategies, the model distinguishes between three states of the battery, it can either be full, good or empty, where the state empty can be interpreted relative to the overall capacity of the battery. The transitions $T_{i}$ for $i \in\{1,2,3,4\}$ coordinate the change of state via test arcs that enable the firing of transition $T_{i}$ according to some threshold that is compared to the available capacity of the battery. Note that whenever the system is in state good, no energy exchange with the grid takes place.

Static continuous transition $T_{\mathcal{C}}$ and dynamic transitions $T_{5}$ and $T_{6}$ are modelling the gird. $T_{5}$ is modelling the flow from the house to the grid, in case the battery is full (when there is a token in the place full) and production is larger than the demand:

$$
r\left(T_{5}\right)=\operatorname{Prod}(t)-\operatorname{Dem}(t) \text { if } \operatorname{Prod}(t)>\operatorname{Dem}(t),
$$

where $\operatorname{Dem}(t)$ and $\operatorname{Prod}(t)$ are the the rate of current working continuous transitions at time $t$, in $\operatorname{Dem}(\mathrm{t})$ and $\operatorname{Prod}(\mathrm{t})$ boxes of Figure A.I, respectively. $T_{6}$ is modelling the flow from the grid to the house in case the battery is in state empty (when there is a token in the place empty):

$$
r\left(T_{6}\right)=\operatorname{Dem}(t)-\operatorname{Prod}(t) \text { if } \operatorname{Prod}(t)<\operatorname{Dem}(t) .
$$


Note that $T_{6}$ is a dynamic transition which does not charge the battery, but only compensates the lack of production to meet the current demand. Finally, static continuous transition $T_{c}$ charges the battery with a constant rate from the grid, when the battery is in the empty state. This transition has a non-zero rate only in case of the conservative strategy.

All thresholds are chosen relative to the overall capacity of the battery $B$. The thresholds for transition $T_{1}$ and $T_{2}$ are used to check whether the current state of charge is smaller-equal or greater-equal than the capacity of battery, respectively. The threshold for $T_{4}$ is used to check whether $B \leq S o C_{1}$ and the threshold for $T_{3}$ checks whether $B>S o C_{1}$ for strategies Greedy and Smart, and checks whether $B>S_{0} C_{2}$ for strategy Conservative. The precise values for the state of charge $S_{o} C_{1}$ and $S_{o} C_{2}$ will be presented later.

The grid failure is modelled as a deterministic transition, $T_{e}$, which can be parametrized through variable $a$. This allows us to analyse the impact of different times of failure on the survivability. The firing of this deterministic transition moves the token to place gridOff and the house is then practically isolated from the grid. The grid returns from its failure according to a stochastic repair distribution that can be chosen arbitrarily.

Finally, we have to note that simultaneous inflow and outflow of energy from the battery in the model is, of course, in reality not possible. One cannot charge and discharge a battery at the same time. In practice, the simultaneous production and demand will bypass the battery. However, the presented model will yield the same results with respect to the chosen measures of interest.

\section{A.2 EVALUATION}

In this section we present the results of the analysis. We first introduce the measure of interest for the given model. Then we characterize the parameters being used for analysis, and finally, we discuss the results for different energy management strategies introduces in the previous section.

\section{A.2.1 Measures of interest}

We again consider the survivability of GOOD models, where a failure (the power outage) is assumed to occur at a certain time $a$. We consider the following Until formula:

$$
\Psi \equiv\left(x_{B} \geq \epsilon\right) \mathcal{U}^{[a, a+t]}\left(m_{\text {gridOn }}=1\right) .^{1}
$$

$\mathbb{\epsilon} \epsilon$ is a positive constant arbitrarily close to zero. 
The above formula then specifies that power is available from the battery continuously until the grid is back on within $t$ time units. We compute the probability to be in a survivable state for a wide range of failure times $a$.

\section{A.2.2 Parameter choices}

In our analysis we consider four different configurations of demand and production profiles: two summer and two winter configurations. The demand and production profiles are already given in Figure A.2b and A.3.

For the four combinations of production and demand, the three battery management strategies have been evaluated for varying usable battery capacities $\left(C_{u}\right)$, ranging from 500 Wh to 3000 Wh. Table A.1 provides the choice for the levels of $S_{o} C_{1}$ and $S_{o} C_{2}$ in the three strategies. The rate at which the battery is charged from the grid in the Conservative strategy, namely the actual rate of the static continuous transition $T_{\mathcal{C}}$, is set to $0.2 C_{u} / 8$. Thus, the battery will be charged by the grid from $0.3 C_{u}$ to $0.5 C_{u}$ in 8 hours. The battery is charged by the grid in such a relatively low rate in order to reduce the additional load on the grid, and to prevent a large number of partial charge-discharge cycles during the night.

Table A.1.: Choice of the threshold levels

\begin{tabular}{l|ccc} 
strategy & $\phi_{S t}^{\mathcal{T}}\left(T_{c}\right)$ & $S_{0} C_{1}$ & $S_{0} C_{2}$ \\
\hline \hline greedy & 0 & 0 & 0 \\
smart & 0 & $0.3 C_{u}$ & $0.3 C_{u}$ \\
conservative & $(.2 / 8) C_{u}$ & $0.3 C_{u}$ & $0.5 C_{u}$
\end{tabular}

\section{A.2.3 Survivability results}

In the following we consider the impact of a grid failure on the smart house for all three battery management strategies and the four production profiles. The grid may fail at different times of the day and come back after a random repair time, that is distributed according to a folded Normal distribution, with average 2 (hours) and standard deviation of 1 (hour). ${ }^{2}$

We show the survivability of the system, that is, the probability that the house can be powered continuously in the presence of a power out-

2 We stress again, that the choice of probability distribution is arbitrary, and can be replaced with any distribution in case of more knowledge about the system being analysed. 
age, for battery sizes between 500 and 3000 Wh. We start with a full battery at midnight (which corresponds to time 0 in the figures).

\section{Greedy strategy}

Figure A.4 presents the survivability of the system, when the Greedy strategy is used for the four different production profiles. The time of failure is depicted on the horizontal axis and the probability that the system is survivable, that is, the probability the demand can be fulfilled without interruptions, on the vertical axis. The time of failure corresponds to the firing time of the failure transition $T_{e}$ in the HPnG model. Clearly, the state of charge of the battery changes over time, hence, the time of failure has a direct impact on the survivability of the system.

In winter when the production is low the available energy from the battery is quickly consumed, since the Greedy strategy always first empties the battery before it imports energy from the grid. Together with the state of charge of the battery the survivability of the system drops rapidly, as shown in Figure A.4a. Depending on the size of the battery, the probability that the system is survivable reaches zero for grid failures occurring between 5 to 15 hours. As the production in this setting is always lower than the demand, the battery will never be recharged on a winter day with low production. Hence, the system can not recover, once the battery has been drained.

The situation changes when the production is higher, e.g., on a sunny winter day. The results for this setting are shown in Figure A.4b and one can see that the survivability is high after 12 p.m., but drops in the evening between 7 p.m. and 9 p.m., depending on the size of the battery. The reason is that the larger production during a sunny afternoon allows to recharge the battery and a full battery provides the means to survive a power outage for a couple of hours. However, during the night and the early morning the battery is always empty due to the Greedy strategy, hence, the probability to survive an outage at these times is zero. The described pattern repeats for consecutive days with this setting. The differences with the first 12 hours are due to the chosen initial condition of the full battery which increases the probability to survive in the beginning.

In summer when the production is low, e.g., on a rainy day, one can see in Figure A.4C that the initial transient phase takes a full day. During this first day the survivability highly depends on the size of the battery, since a larger capacity provides more backup in case of a grid failure. However, after the battery has been emptied once (after the first 24 hours) one can see that the survivability is independent of the battery capacity. The reason is that although the battery is charged during the day, due to the low production, it is never charged above $600 \mathrm{Wh}$. Hence, 

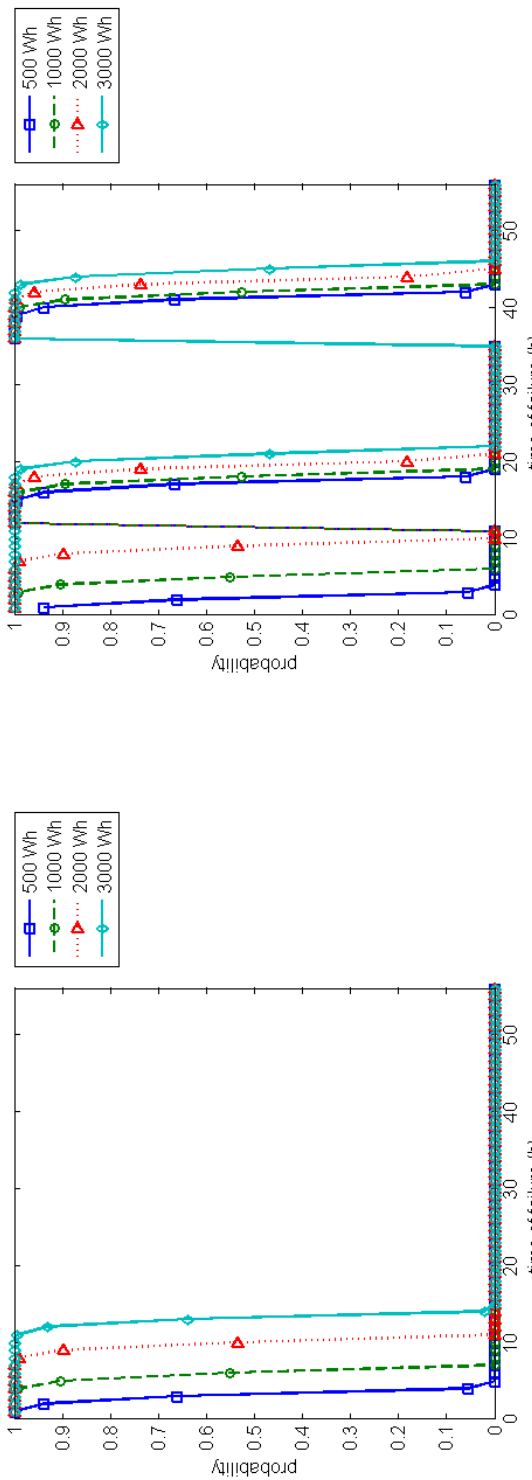
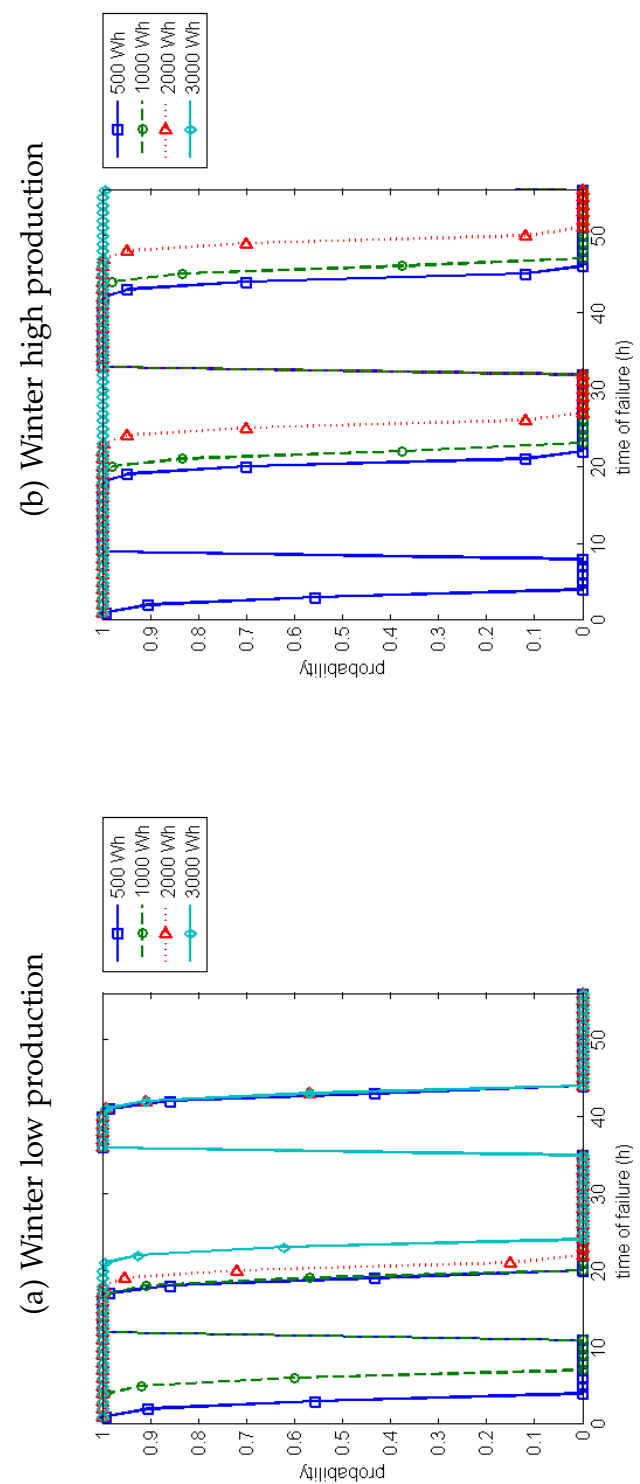



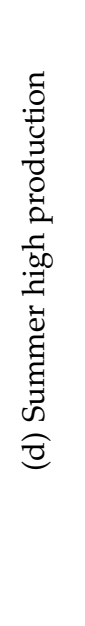

疋

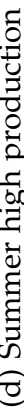

ర్ 
additional battery capacity does not have an advantage with respect to the survivability.

On a summer day with high production (Figure A.4d) the start-up phase is much shorter, i.e., less than 10 hours. The reason is that the production is so high that an empty battery is quickly charged in the morning. It is interesting to see that in this setting, with the highest battery capacity of $3000 \mathrm{Wh}$, the system is survivable with probability one for all considered failure times. With the battery of $2000 \mathrm{Wh}$ the system is not survivable for a couple of hours during the night. Hence, in this setting it is preferable to have a larger battery, while on a summer day with a low production the additional capacity does not increase the survivability in the long run.

Overall, the Greedy strategy results in a poor survivability for three out of four production profiles, i.e., with the exception of a summer day with high production. In all other cases the complete draining of the battery leads to a zero probability to survive a power outage for large parts of the day. In the following we will look at the two remaining strategies, Smart and Conservative, for the two settings Winter low and Summer high to contrast the impact of the production profiles on the battery management strategies.

\section{Smart strategy}

Figure A.5 shows the survivability of the system when the Smart strategy is used. Recall that this strategy never drains the battery completely while the grid is available and always reserves $30 \%$ of the battery capacity to survive power outages. On a winter day with low production, as depicted in Figure A.5a, the survivability highly depends on the overall capacity of the battery. With this strategy the largest battery ensures a survivability of at least $70 \%$ for failures occurring at all times of the day, which is a large improvement with respect to the greedy strategy, where the survivability was zero, once the battery had been drained. When a smaller battery, e.g., $500 \mathrm{Wh}$, is used, the survivability drops to $10 \%$ during the night, which is clearly very low. This figure also exhibits a start-up phase, which is, however, rather small (less then 10 hours), after which a pattern repeats with a high survivability during the day and a dip during the evening hours. It is interesting to see that the survivability increases during the night even though the battery cannot be charged with local energy and will not be charged from the grid due to the strategy employed. This is due to the drop of the demand during the night, hence, the system can survive longer on the remaining $30 \%$ battery capacity than during peak evening hours.

On a summer day with high production (cf. Figure A.5b) the two largest batteries lead to a survivability of $100 \%$ for all potential failure occurrence times. For the two smaller batteries a pattern emerges after 


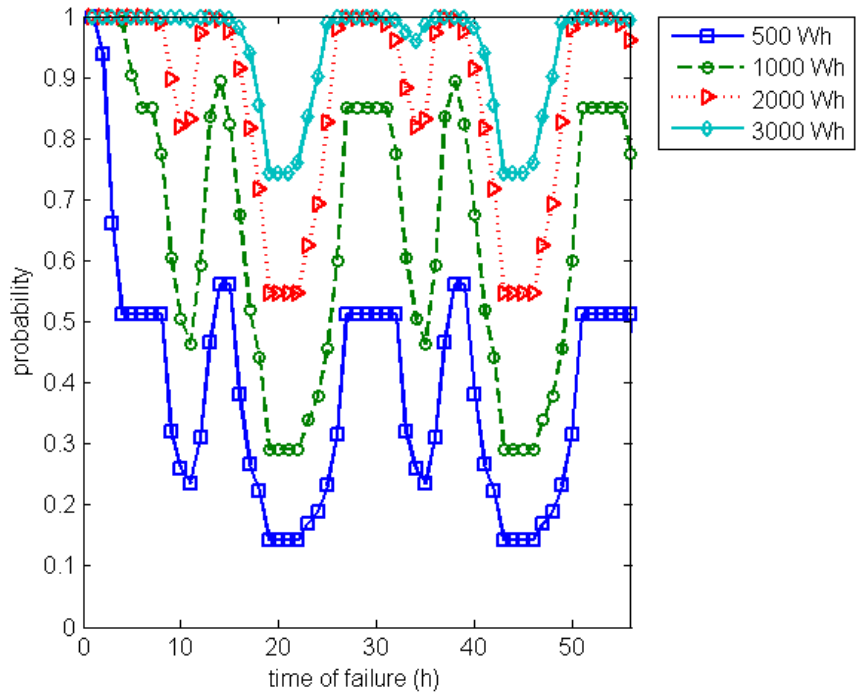

(a) Winter low production

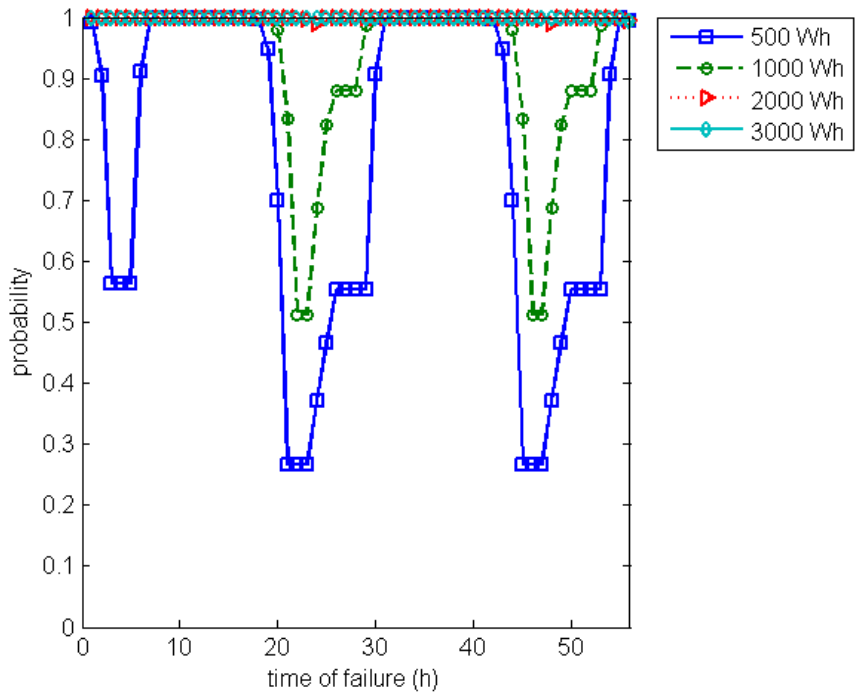

(b) Summer high production

Figure A.5.: Probability of surviving a grid failure as function of the time that the failure occurs for the Smart strategy. 
the initial start-up time of 10 hours, where the survivability is again high during the day but drops during the evening hours when the demand is very high and local energy is not available. During the night, the survivability then again increases due to the decreasing demand and returns to one as soon as the local generation produces energy during the day.

When comparing the two strategies presented so far, the minimum survivability with the Smart strategy is, depending on the size of the battery, much better than when Greedy is used. However, the time intervals where the survivability is less good remain the same.

\section{Conservative strategy}

Finally, we also analyse the Conservative strategy, where the resilience of the system is increased by additionally charging the battery from the grid, when its state of charge is lower than the predefined threshold $S_{o} C_{2}$. The resulting survivability is presented in Figure A.6. Especially on a winter day with low production, see Figure A.6a, the variability is much higher than in the other settings. The reason is that we observe a relatively high number of state changes between the states good and empty of the battery management unit. As soon as the state of charge is less than $30 \%$ of the overall battery capacity, the grid is used to power the house, and it also charges the battery until it reaches $50 \%$ of its capacity. If this occurs at a point in time where the local generation is still not producing enough energy, the house is then powered from the battery until again the state of charge hits the 30\% threshold. Especially for smaller battery sizes these state changes occur relatively often since the difference between $30 \%$ and $50 \%$ state of charge is smaller. As a result of the large amount of variability in the system, also the results do not reveal a clear pattern for the different battery sizes.

On a summer day with high production, the survivability is much more regular, as shown in Figure A.6b. The start-up phase takes about 10 hours and we observe a survivability of one for the two large batteries and a minimum survivability of $30 \%$ and $55 \%$ for battery sizes $500 \mathrm{Wh}$ and $1000 \mathrm{Wh}$, respectively, during the evening dips. When compared to the results for the Smart strategy one sees that the time intervals where the survivability is relatively low are much smaller for the Conservative strategy.

Overall one can conclude that achieving a high survivability is especially difficult on a winter day with low production. Having a larger battery only increases the survivability in this setting if one reserves backup power in the battery, that is only used in the case of a power outage. Additionally charging the battery from the grid in case of a low state of charge also improves the overall survivability and decreases the intervals of time where the survivability is low. 


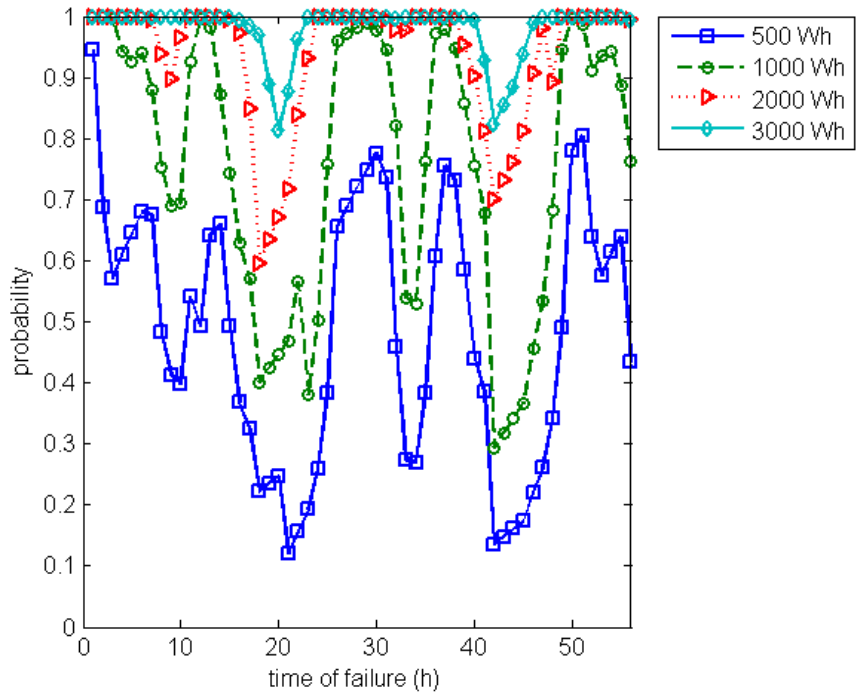

(a) Winter low production

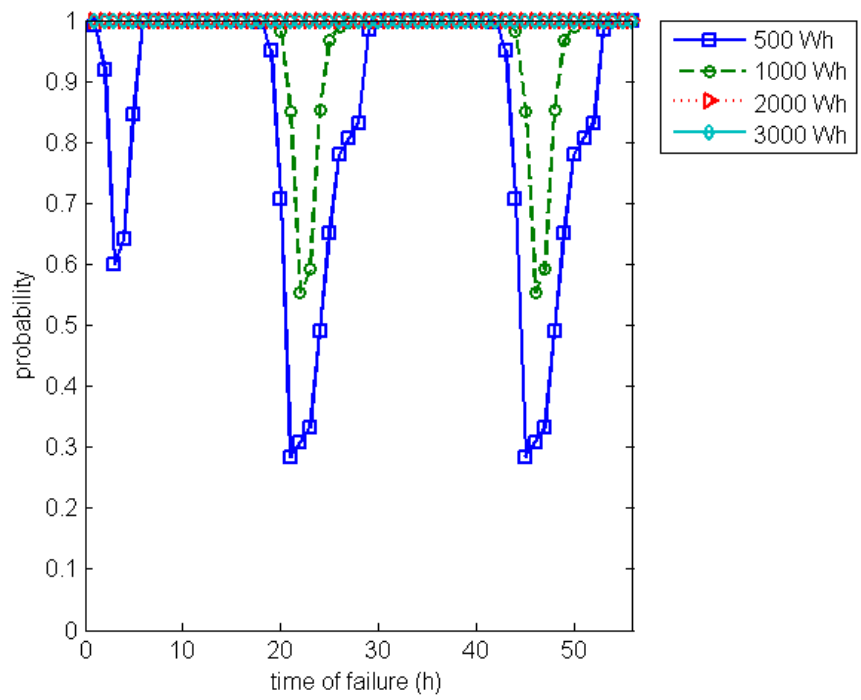

(b) Summer high production

Figure A.6.: Probability of surviving a grid failure as function of the time that the failure occurs for the Conservative strategy. 
In contrast, on a summer day with high production the two large batteries are enough to ensure a survivability of $100 \%$ for the strategies Smart and Conservative, and even for Greedy the largest battery capacity ensures the same. One can conclude that in order to ensure a relatively high survivability, a smart house with the presented parameter settings would need a battery with at least a capacity of $2000 \mathrm{Wh}$ and would employ at least strategy Smart. Note that, when the Conservative strategy is used, the thresholds $S_{o} C_{1}, S_{o} C_{2}$ and the additional charge from the grid have to be chosen carefully to reduce the number of state changes in the battery management unit, hence, to avoid cycling behaviour.

\section{A.2.4 Computation times}

To obtain the presented results, for each scenario, i.e., for each combination of production profile and battery management strategy, we have analyzed 248 different settings, each with a different overall battery capacity and failure time. For each of of these 248 combinations, a different STD needs to be generated and model checked in order to compute the required measure of survivability. The analyses have been performed on a machine equipped with a $2.0 \mathrm{GHz}$ intel CORE i7 processor and $4 \mathrm{~GB}$ of RAM.

The computations for each setting have taken at most half a second. To compute all 248 values for each scenario has taken a maximum of 194 seconds for the conservative strategy with a winter-low production profile (and less for all other scenarios). Moreover, the maximum number of generated regions for a STD has been slightly less that 7200. More details on the computation times and the number of generated regions are given in Table A.2.

\section{A.3 RELATED WORK}

Smart grids are highly complex systems [117], controlled by an ICT network that is used to exchange information. The electricity grid consists of multiple voltage levels and generated electricity is fed in on different voltage levels, where transformers convert energy between voltage levels. In order to model the stability of the grid, electricity demand, power generation, grid losses, the capacity and loss in transformers, as well as renewable generation need to be taken into account [118]. Most work on modelling of smart grids focuses on optimization [119, 120], stability issues [121] or presents relatively simple Petri net models for dependability assessment [122].

Work specifically on grid resilience has been done in the past, however, thereby primarily focussing on the resilience of the high- and mediumvoltage grid. In these studies, the impact of failures, e.g., due to thunder- 
Table A.2.: Computation times and model size.

\begin{tabular}{cc|ccc} 
strategy & $\begin{array}{c}\text { production } \\
\text { profile }\end{array}$ & $\begin{array}{c}\text { total comp. } \\
\text { time (s) }\end{array}$ & $\begin{array}{c}\text { max } \\
\text { \# regions }\end{array}$ & $\begin{array}{c}\text { average } \\
\text { \# regions }\end{array}$ \\
\hline \hline \multirow{4}{*}{ greedy } & winter low & 23.7 & 573 & 182 \\
& winter high & 43.4 & 1384 & 402 \\
& summer low & 29.4 & 1099 & 239 \\
& summer high & 46.9 & 1410 & 401 \\
\hline \multirow{5}{*}{ smart } & winter low & 24.7 & 563 & 189 \\
& winter high & 51.2 & 1607 & 402 \\
& summer low & 36.6 & 1365 & 293 \\
& summer high & 60.3 & 1735 & 524 \\
\hline \multirow{5}{*}{ conservative } & winter low & 194.0 & 7188 & 1927 \\
& winter high & 129.5 & 5245 & 1331 \\
& summer low & 134.8 & 6106 & 1266 \\
& summer high & 109.5 & 5844 & 1021
\end{tabular}

storms, is studied in terms of the (expected) energy not supplied per unit of time, see, e.g., [123,124]. The proposed models are primarily based on Markov chains, stochastic activity networks and Markov reward models; numerical analysis or simulation is used for evaluation purposes. The proposed models for grid resilience have a discrete state space and, typically, the expected energy not supplied (per unit time) is computed (or estimated) using a time-scale decomposition. A recent overview of this type of work, but also related work for the gas and water infrastructure can be found in [7].

All these works take the standpoint of the grid operator and/or the energy supplier. Instead, the analysis proposed in this chapter is focused on the viewpoint of the end-user. For this we studied the continuity of energy delivery to end-user appliances irrespective of what happens exactly in the grid. In our modelling approach, the grid is just seen as a source of energy delivery, albeit with possible interruptions. Our approach allows us to study the impact of grid disruptions, for whatever reason, on end-user perceived energy delivery continuity, and how we can improve the perceived continuity of energy delivery by installing local storage. The models we used are truly stochastic hybrid models, in that they combine deterministic events with probabilistic events on a mixed discrete/continuous state space. 

[1] M. De Bruijne and M. Van Eeten. Systems that should have failed: critical infrastructure protection in an institutionally fragmented environment. Journal of Contingencies and Crisis Management, 15(1):18-29, 2007.

[2] J. Johansson, H. Hassel, and E. Zio. Reliability and vulnerability analyses of critical infrastructures: comparing two approaches in the context of power systems. Reliability Engineering $\mathcal{E}$ System Safety, 120:27-38, 2013.

[3] C. Baier and J-P. Katoen. Principles of model checking. MIT press, 2008.

[4] L. Cloth and B.R. Haverkort. Model checking for survivability! In The second International Conference on the Quantitative Evaluation of Systems, 2005, pages 145-154. IEEE, 2005.

[5] P. E. Heegaard and K. S. Trivedi. Network survivability modeling. Computer Networks, 53(8):1215-1234, 2009.

[6] J. C. Knight and K. Sullivan. On the definition of survivability. Technical report, University of Virginia, 2000.

[7] A. Avritzer, L. Carnevali, H. Ghasemieh, L. Happe, B. R. Haverkort, A. Koziolek, D. Menasche, A. Remke, S. Sedigh Sarvestani, and E. Vicario. Survivability evaluation of gas, water and electricity infrastructures. Electronic Notes in Theoretical Computer Science, 310:5-25, 2015.

[8] M. H. Davis. Markov Models E Optimization. CRC Press, 1993.

[9] M. K. Ghosh, A. Arapostathis, and S. I. Marcus. Ergodic control of switching diffusions. SIAM Journal on Control and Optimization, 35(6):1952-1988, 1997 .

[10] J. Hu, J. Lygeros, and S. Sastry. Towards a theory of stochastic hybrid systems. In Hybrid Systems: Computation and Control, volume 1790 of LNCS, pages 160-173. Springer, 2000.

[11] M. Kwiatkowska, G. Norman, R. Segala, and J. Sproston. Automatic verification of real-time systems with discrete probability distributions. Theoretical Computer Science, 282:101-150, 2002. 
[12] M. Kwiatkowska, G. Norman, R. Segala, and J. Sproston. Verifying quantitative properties of continuous probabilistic timed automata. In International Conference on Concurrency Theory, volume 1877 of LNCS, pages 123-137. 2000.

[13] G. Pola, M.L. Bujorianu, J. Lygeros, and M.D. Di Benedetto. Stochastic hybrid models: An overview. In IFAC Conference on Analysis and Design of Hybrid Systems, pages 45-50, 2003.

[14] J. Sproston. Decidable Model Checking of Probabilistic Hybrid Automata. In Formal Techniques in Real-Time and Fault-Tolerant Systems, volume 1926 of LNCS, pages 31-45. Springer, 2000.

[15] A. Abate, M. Prandini, J. Lygeros, and S. Sastry. Probabilistic reachability and safety for controlled discrete time stochastic hybrid systems. Automatica, 44(11):2724-2734, 2008.

[16] G. Horton, V.G. Kulkarni, D.M. Nicol, and K.S. Trivedi. Fluid stochastic Petri nets: Theory, applications, and solution techniques. European Journal of Operational Research, 105(1):184-201, 1998.

[17] R. Alur, C. Courcoubetis, N. Halbwachs, T. Henzinger, S. Yovine, P.H. Ho, X. Nicollin, A. Olivero, and J. Sifakis. The algorithmic analysis of hybrid systems. Theoretical Computer Science, 138:3-34, 1995 .

[18] R. Alur, C. Courcoubetis, and T. Henzinger. Hybrid automata: An algorithmic approach to the specification and verification of hybrid systems. Hybrid Systems, 736:209-229, 1993.

[19] T.A. Henzinger. The theory of hybrid automata. In 11th Annual IEEE Symposium on Logic in Computer Science, pages 278-292. IEEE, 1996.

[20] R. David and H. Alla. On hybrid Petri nets. Discrete Event Dynamic Systems, 3:9-40, 2001.

[21] M. Ajmone Marsan, G. Conte, and G. Balbo. A class of generalized stochastic Petri nets for the performance evaluation of multiprocessor systems. ACM Transactions on Computer Systems, 2(2):93-122, 1984.

[22] G. Ciardo and R. German. A characterization of the stochastic process underlying a stochastic Petri net. IEEE Transactions on Software Engineering, 20:506-515, 1994.

[23] R. German and C. Lindemann. Analysis of stochastic Petri nets by the method of supplementary variables. Performance Evaluation, 20(1-3):317-335, 1994 . 
[24] K. S. Trivedi and V. Kulkarni. FSPNs: fluid stochastic Petri nets. In International Conference on Application and Theory of Petri Nets, volume 691 of LNCS, pages 24-31. Springer, 1993.

[25] K. Wolter, G. Horton, and R. German. Non-Markovian fluid stochastic Petri nets. Technical report, Technische Universität Berlin, 1996.

[26] M. Gribaudo and A. Remke. Hybrid Petri nets with general oneshot transitions for dependability evaluation of fluid critical infrastructures. In 12th International Symposium on High Assurance Systems Engineering, pages 84-93. IEEE, 2010.

[27] G. Ciardo, D.M. Nicol, and K.S. Trivedi. Discrete-event simulation of fluid stochastic Petri nets. IEEE Transactions on Software Engineering, 25(2):207-217, 1999.

[28] M. Marsan, G. Balbo, G. Conte, S. Donatelli, and G. Franceschinis. Modelling with Generalized Stochastic Petri Nets. John Wiley \& Sons, Inc, 1994.

[29] M. K. Molloy. Performance analysis using stochastic Petri nets. IEEE Transactions on Computers, 100(9):913-917, 1982.

[30] M. K. Molloy. On the integration of delay and throughput measures in distributed processing models. PhD thesis, University of California, Los Angele, 1981.

[31] M. Ajmone Marsan and G. Chiola. On Petri nets with deterministic and exponentially distributed firing times. In European Workshop on Applications and Theory in Petri Nets, volume 266 of LNCS, pages 132-145. Springer, 1986.

[32] H. Choi and V.G. Kulkarni. Markov regenerative stochastic Petri nets. Performance evaluation, 20(1):337-357, 1994.

[33] M. Telek and A. Horváth. Supplementary variable approach applied to the transient analysis of age-MRSPNs. In Computer Performance and Dependability Symposium, pages 44-51. IEEE, 1998.

[34] R. David and H. Alla. Discrete, Continuous, and Hybrid Petri Nets. Springer, 2010.

[35] M.K. Molloy. Discrete Time Stochastic Petri Nets. IEEE Transactions on Software Engineering, 11(4):417-423, 1985.

[36] C. A. Petri and W. Reisig. Petri net. Scholarpedia, 3(4):6477, 2008.

[37] C. A. Petri. Kommunikation mit Automaten. PhD thesis, Universität Hamburg, 1962. 
[38] C. A. Petri. Introduction to general net theory. In Advanced Course on General net Theory of Processes and Systems: Net Theory and Applications, pages 1-19. Springer-Verlag, 1980.

[39] C. Ramchandani. Analysis of asynchronous concurrent systems by timed Petri nets. Technical report, Massachusetts Institute of Technology, 1974.

[40] W. Zuberek. Timed Petri nets and preliminary performance evaluation. In $7^{\text {th }}$ annual symposium on Computer Architecture, pages 88-96. ACM, 1980.

[41] R. Razouk and C. Phelps. Performance analysis using timed Petri nets. In Protocol Specification, Testing, and Verification, volume 84, pages $561-576,1984$.

[42] J. Sifakis. Use of Petri nets for performance evaluation. Acta Cybernetica, 4(1978):185-202, 1980.

[43] T. Agerwala and M. Flynn. Comments on capabilities, limitations and "correctness" of Petri nets. ACM Computer Architecture News, 2(4):81-86, 1973 .

[44] M. Hack. Extended state-machine allocatable nets (ESMA): An extension of free choice Petri net results. Massachusetts Institute of Technology Project MAC, 1974 .

[45] Aristotle's Physics. MIT classics. R. P. Hardie and R. K. Gaye.

[46] R. Omnès. Quantum philosophy: understanding and interpreting contemporary science. Princeton University Press, 2002.

[47] R. David and H. Alla. Continuous Petri nets. In 8th European Workshop on Application and Theory of Petri nets, volume 340, pages 275-294, 1987.

[48] R. David and H. Alla. Autonomous and timed continuous Petri nets. In Advances in Petri Nets 1993, LNCS, pages 71-90. Springer, 1991.

[49] F. Balduzzi, A. Giua, and G. Menga. Hybrid stochastic Petri nets: firing speed computation and FMS modelling. In 4th Workshop on Discrete Event Systems, pages 432-438, 1998.

[50] F. Balduzzi, A. Giua, and G. Menga. First-order hybrid Petri nets: a model for optimization and control. IEEE Transactions on Robotics and Automation, 16(4):382-399, 2000. 
[51] H. Ghasemieh, A. Remke, B.R. Haverkort, and M Gribaudo. Region-based analysis of hybrid Petri nets with a single general one-shot transition. In Formal Modeling and Analysis of Timed Systems, volume 7595 of LNCS, pages 139-154. Springer, 2012.

[52] H. Ghasemieh, A. Remke, and B. R. Haverkort. Survivability evaluation of fluid critical infrastructures using hybrid Petri nets. In 19th Pacific Rim International Symposium on Dependable Computing, pages 152-161. IEEE, 2013.

[53] R. Alur and D.L. Dill. A theory of timed automata. Theoretical Computer Science, 126(2):183-235, 1994.

[54] E. Vicario, L. Sassoli, and L. Carnevali. Using stochastic state classes in quantitative evaluation of dense-time reactive systems. IEEE Transactions on Software Engineering, 35(5):703-719, 2009.

[55] E. Vicario. Static analysis and dynamic steering of time-dependent systems. IEEE Transactions on Software Engineering, 27(8):728-748, 2001.

[56] B. Berthomieu. Modeling and verification of time dependent systems using time Petri nets. IEEE Transactions on Software Engineering, 17(3):259-273, 1991.

[57] E. Asarin and O. Maler. Reachability analysis of dynamical systems having piecewise-constant derivatives. Theoretical computer science, 138(1):35-65, 1995.

[58] Z. Manna and A. Pnueli. Temporal verification of reactive systems: safety. Springer Science \& Business Media, 2012.

[59] A. Pnueli. The temporal logic of programs. In 18th Annual Symposium on Foundations of Computer Science, pages 46-57. IEEE, 1977.

[6o] E. Clarke and A. Emerson. Design and synthesis of synchronization skeletons using branching time temporal logic. In Workshop on Logic of Programs, pages 52-71. Springer, 1981.

[61] R. Alur, T. Feder, and T. Henzinger. The benefits of relaxing punctuality. Journal of the ACM, 43(1):116-146, 1996.

[62] O. Maler and D. Nickovic. Monitoring temporal properties of continuous signals. In Formal Techniques, Modelling and Analysis of Timed and Fault-Tolerant Systems, volume 3253 of LNCS, pages 152-166. Springer, 2004.

[63] H. Ghasemieh, A. Remke, and B. R. Haverkort. Hybrid Petri nets with multiple stochastic transition firings. In 8th International Conference on Performance Evaluation Methodologies and Tools, pages 217224. ICST, 2014. 
[64] F.P. Preparata and D.E. Muller. Finding the intersection of $n$ halfspaces in time $o(n \log n)$. Theoretical Computer Science, 8(1):45-55, 1979.

[65] D. Halperin. Arrangements. In Handbook of Discrete and Computational Geometry, pages 529-562. CRC Press LLC, 2004.

[66] M. de Berg, O. Cheong, M. van Kreveld, and M. Overmars. Computational Geometry: Algorithms and Applications. Springer, 3rd edition, 2008.

[67] A. Blakemore. The cost of eliminating vanishing markings from generalized stochastic Petri nets. In Third International Workshop on Petri Nets and Performance Models, pages 85-92. IEEE, 1989.

[68] G. Ciardo. Analysis of large stochastic Petri net models. PhD thesis, Duke University, 1990.

[69] A. Bell. Distributed evaluation of stochastic Petri nets. PhD thesis, RWTH Aachen University, 2004.

[70] H. Edelsbrunner, J. O'Rourke, and R. Seidel. Constructing arrangements of lines and hyperplanes with applications. SIAM Journal on Computing, 15(2):341-363, 1986.

[71] S. Hert and S. Schirra. 3D convex hulls. In CGAL User and Reference Manual. CGAL Editorial Board, 2014.

[72] S Hert and M Seel. dD convex hulls and Delaunay triangulations. In CGAL User and Reference Manual. CGAL Editorial Board, 2014.

[73] R. Wein, E Berberich, E. Fogel, D. Halperin, M. Hemmer, O. Salzman, and B. Zukerman. 2D arrangements. In CGAL User and Reference Manual. CGAL Editorial Board, 2014.

[74] S. Abate, A.and Amin, M. Prandini, J. Lygeros, and S. Sastry. Computational approaches to reachability analysis of stochastic hybrid systems. In International Workshop on Hybrid Systems: Computation and Control, pages 4-17. Springer, 2007.

[75] A. L. Visintini, W. Glover, J. Lygeros, and J. Maciejowski. Monte Carlo optimization for conflict resolution in air traffic control. IEEE Transactions on Intelligent Transportation Systems, 7(4):470-482, 2006.

[76] A. Abate, J.-P. Katoen, J. Lygeros, and M. Prandini. Approximate model checking of stochastic hybrid systems. European Journal of Control, 16:624-641, 2010.

[77] E. Fogel, R. Wein, B. Zukerman, and D. Halperin. 2D regularized Boolean set-operations. In CGAL User and Reference Manual. CGAL Editorial Board, 2014. 
[78] P. Hachenberger and L. Kettner. 3 D Boolean operations on Nef polyhedra. In CGAL User and Reference Manual. CGAL Editorial Board, 2014.

[79] M. Galassi, J. Davies, J. Theiler, B. Gough, G. Jungman, P. Alken, M. Booth, F. Rossi, and R. Ulerich. GNU Scientific Library. 2013.

[8o] K. Weiler and P. Atherton. Hidden surface removal using polygon area sorting. In Computer Graphics, pages 214-222, 1977.

[81] B. Vatti. A generic solution to polygon clipping. Communications of the ACM, 35(7):56-63, 1992.

[82] G. Greiner and K. Hormann. Efficient clipping of arbitrary polygons. ACM Transactions on Graphics, 17(2):71-83, 1998.

[83] G. Giezeman and W. Wesselink. 2D polygons. In CGAL User and Reference Manual. CGAL Editorial Board, 4.8 edition, 2016.

[84] T.A. Henzinger, P. Ho, and H. Wong-Toi. HyTech: A model checker for hybrid systems. Software Tools for Technology Transfer, 1(1-2):110-122, 1997.

[85] G. Frehse. PHAVer: Algorithmic verification of hybrid systems past HyTech. In Hybrid Systems: Computation and Control, volume 3414 of LNCS, pages 258-273. Springer, 2005.

[86] G. Frehse, C. Le Guernic, A. Donze, R.R.O. Lebeltel, R. Ripado, A. Girard, T. Dang, and O. Maler. SpaceEx: Scalable verification of hybrid systems. In International Conference on Computer Aided Verification, volume 6806 of LNCS, pages 379-395. Springer, 2011.

[87] E. M. Hahn, A. Hartmanns, H. Hermanns, and J.-P. Katoen. A compositional modelling and analysis framework for stochastic hybrid systems. Springer Formal Methods in System Design, 43(2):191-232, 2013.

[88] L. Zhang, Z. She, S. Ratschan, H. Hermanns, and E. Hahn. Safety verification for probabilistic hybrid systems. In Computer Aided Verification, volume 6174 of LNCS, pages 196-211. Springer, 2010.

[89] A.A. Julius. Approximate abstraction of stochastic hybrid automata. In Hybrid Systems: Computation and Control, volume 3927 of LNCS, pages 318-332. Springer, 2006.

[9o] M. Bujorianu, J. Lygeros, and R. Langerak. Reachability analysis of stochastic hybrid systems by optimal control. In Hybrid Systems: Computation and Control, volume 4981 of LNCS, pages 610613. Springer, 2008. 
[91] M. Gribaudo, A. Horvath, A Bobbio, E. Tronci, E. Ciancamerla, and M. Minichino. Fluid Petri nets and hybrid model-checking: a comparative case study. Reliability Engineering and System Safety, 81(3):239-257, 2003.

[92] H. Ghasemieh, A. Remke, and B. R. Haverkort. Survivability analysis of a sewage treatment facility using hybrid Petri nets. Performance evaluation, 97:36-56, 2016.

[93] H. Ghasemieh, A. Remke, B. R. Haverkort, and G. Ciardo. Approximate analysis of hybrid Petri nets with probabilistic timed transitions. In 9th International Conference on Performance Evaluation Methodologies and Tools, pages 127-134. ICST, 2016.

[94] N. Metropolis and S. Ulam. The Monte Carlo method. Journal of the American Statistical Association, 44(247):335-341, 1949.

[95] J. Pearl. Heuristics: intelligent search strategies for computer problem solving. Addison-Wesley, 1984.

[96] P. E. Hart, N. J. Nilsson, and B. Raphael. A formal basis for the heuristic determination of minimum cost paths. Systems Science and Cybernetics, IEEE Transactions on, 4(2):100-107, 1968.

[97] T. H. Cormen, C. E. Leiserso, R. L. Rivest, and C. Stein. Introduction to Algorithms. The MIT Press, 1990.

[98] M. Wan and G. Ciardo. Symbolic reachability analysis of integer timed Petri nets. In Theory and Practice of Computer Science, volume 5404 of LNCS, pages 595-608. Springer, 2009.

[99] A.P.A. Van Moorsel and B.R. Haverkort. Probabilistic evaluation for the analytical solution of large Markov models: Algorithms and tool support. Microelectronics Reliability, 36(6):733 - 755, 1996.

[10o] A. Remke, B.R. Haverkort, and L. Cloth. CSL model checking algorithms for QBDs. Theoretical Computer Science, 382(1):24 - 41, 2007.

[101] D. Klink, A. Remke, B. R. Haverkort, and J.-P. Katoen. Timebounded reachability in tree-structured QBDs by abstraction. Performance Evaluation, 68(2):105-125, 2011.

[102] H. Ghasemieh, A. Remke, and B. R. Haverkort. Analysis of a sewage treatment facility using hybrid Petri nets. In 7 th International Conference on Performance Evaluation Methodologies and Tools, pages 165-174. ICST, 2013.

[103] RTV Oost. Overijssel Vandaag, July 2013. http://www.rtvoost.nl/ tv/uitzendinggemist.aspx?uid=290892, Accessed January 18, 2017. 
[104] TV Enschede FM. TV Enschede Nieuws, June 2013. http:// www.youtube.com/watch?v=DRIB6JTNvhA, Accessed January 18, 2017.

[105] S. M. Khopkar. Environmental Pollution Monitoring and Control. New Age International, 2004.

[106] Primer for municipal wastewater treatment systems, 2004. http:// www.epa.gov/npdes/pubs/primer.pdf, Accessed January 18, 2017.

[107] M. Ohnari. Simulation Engineering. IOS press, 1998.

[108] Meidensha Corporation. Sewage treatment process simulator. http://www.meidensha.com/products/water/prod_04/prod_04_ 01/index.html, Accessed January 18, 2017.

[109] H. Abarbanel, S. Koonin, H. Levine, G. MacDonald, and O. Rothaus. Statistics of extreme events with application to climate. Technical report, DTIC Document, 1992.

[110] H. Ghasemieh, B. R. Haverkort, M. R. Jongerden, and A. Remke. Energy resilience modelling for smart houses. In 45th Annual IEEE/IFIP International Conference on Dependable Systems and Networks, pages 275-286. IEEE, 2015.

[111] Renewable energy: Puffs of hope. Economist, pages 9-10, August 1st, 2015.

[112] Nedap. PowerRouter homepage. http://www.powerrouter.com/ en/, Accessed January 18, 2017.

[113] SMA. SMA homepage. http://www.sma.de/en.html, Accessed January $18,2017$.

[114] EDSN. EDSN demand profiles. http://www.edsn.nl/ verbruiksprofielen/, Accessed January 18, 2017.

[115] NREL. PVWatts. http://pvwatts.nrel.gov/index.php, Accessed January $18,2017$.

[116] M. Jongerden and B. Haverkort. Which battery model to use? IET Software, 3(6):445-457, 2009.

[117] S. M. Amin. Smart grid: Overview, issues and opportunities. advances and challenges in sensing, modeling, simulation, optimization and control. European Journal of Control, 17(56):547 - 567, 2011.

[118] H. Hermanns and H. Wiechmann. Future design challenges for electric energy supply. In IEEE Conference on Emerging Technologies \& Factory Automation, pages 1-8. IEEE, 2009. 
[119] V. Bakker, M. G.C. Bosman, A. Molderink, J. L. Hurink, and G. J.M. Smit. Improved heat demand prediction of individual households. In First Conference on Control Methodologies and Technology for Energy Efficiency, pages 110-115. Elsevier, 2010.

[120] M. Welsch, M. Howells, M. Bazilian, J.F. DeCarolis, S. Hermann, and H.H. Rogner. Modelling elements of smart grids enhancing the OSeMOSYS (open source energy modelling system) code. Energy, 46(1):337 - 350, 2012.

[121] A. Hartmanns and H. Hermanns. Modelling and decentralised runtime control of self-stabilising power micro grids. In Proccesdings of the 5th International Symposium Leveraging Applications of Formal Methods, Verification and Validation. Technologies for Mastering Change, volume 7609 of LNCS, pages 420-439. Springer, 2012.

[122] B.C. Wang, M. Sechilariu, and F. Locment. Power flow Petri net modelling for building integrated multi-source power system with smart grid interaction. Mathematics and Computers in Simulation, 91(0):119 - 133, 2013.

[123] A. Alberto, L. Carnevali, L. Happe, A. Koziolek, D. Sadoc Menasché, M. Paolieri, and S. Suresh. A scalable approach to the assessment of storm impact in distributed automation power grids. In 11th International Conference on Quantitative Evaluation of Systems, volume 8657 of LNCS, pages 345-367. Springer, 2014.

[124] S. Chiaradonna, F. Di Giandomenico, and N. Murru. On a modeling approach to analyze resilience of a smart grid infrastructure. In 1oth European Dependable Computing Conference, pages 166-177. IEEE, 2014. 
"Without education, we are in a horrible and deadly danger of taking educated people seriously."

- G. K. Chesterton

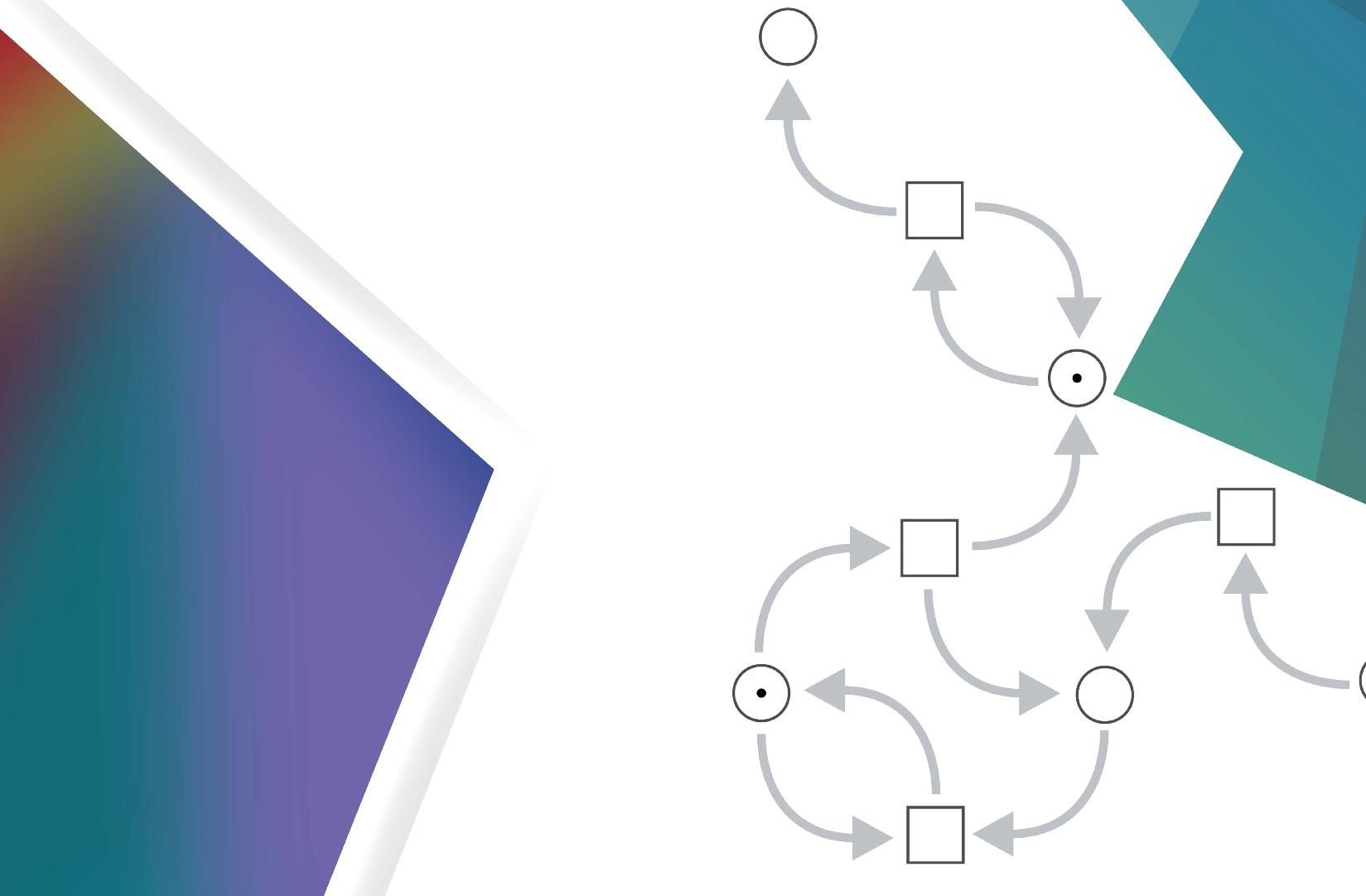

ISBN: 978-90-365-4257-9 